\title{
Physics of Diurnal Warm Layers: \\ Turbulence, Internal Waves, and Lateral Mixing
}

by

\author{
Alec Setnor Bogdanoff \\ B.S. in Meteorology, Florida State University, 2008 \\ M.S. in Meteorology, Florida State University, 2010 \\ Submitted in partial fulfillment of the requirements for the degree of \\ Doctor of Philosophy \\ at the \\ MASSACHUSETTS INSTITUTE OF TECHNOLOGY \\ and the \\ WOODS HOLE OCEANOGRAPHIC INSTITUTION
}

February 2017

(c) 2017 Alec Setnor Bogdanoff. All rights reserved.

The author hereby grants to MIT and WHOI permission to reproduce and to distribute publicly paper and electronic copies of this thesis document

in whole or in part in any medium now known or hereafter created.

Author

Joint Program in Oceanography/Applied Ocean Science and Engineering Massachusetts Institute of Technology and Woods Hole Oceanographic Institution

November 3, 2016

Certified by

J. Thomas Farrar Associate Scientist, Woods Hole Oceanographic Institution Thesis Co-Supervisor

Certified by

Carol Anne Clayson

Senior Scientist, Woods Hole Oceanographic Institution

Thesis Co-Supervisor

Accepted by

Lawrence J. Pratt

Senior Scientist, Woods Hole Oceanographic Institution Chairperson, Joint Committee for Physical Oceanography 


\title{
Physics of Diurnal Warm Layers: Turbulence, Internal Waves, and Lateral Mixing \\ by
}

\author{
Alec Setnor Bogdanoff
}

Submitted to the Joint Program in Oceanography/Applied Ocean Science and Engineering Massachusetts Institute of Technology and Woods Hole Oceanographic Institution on November 3, 2016, in partial fulfillment of the requirements for the degree of Doctor of Philosophy

\begin{abstract}
The daily heating of the ocean by the sun can create a stably stratified near-surface layer when the winds are slight and solar insolation is strong. This type of shallow stable layer is called a Diurnal Warm Layer (DWL). This thesis examines the physics and dynamics of DWLs from observations of the subtropical North Atlantic Ocean associated with the Salinity Processes in the Upper ocean Regional Study (SPURS-I).

Momentum transferred from the atmosphere to the ocean through wind stress becomes trapped within the DWL, generating shear across the layer. During SPURS-I, strong diurnal shear across the DWL was coincident with enhanced turbulent kinetic energy (TKE) dissipation $\left(\epsilon, \epsilon>10^{-5} \mathrm{~W} / \mathrm{kg}\right.$ ) observed from glider microstructure profiles of the near-surface. However, a scale analysis demonstrated that surface forcing, including diurnal shear, could not be the sole mechanism for the enhanced TKE dissipation.

High-frequency internal waves $(\omega \gg f)$ were observed in the upper ocean during the daytime within the DWL. Internal waves are able to transfer energy from the deep ocean into the DWL through the unstratified remnant mixed layer, which is the intervening layer between the DWL and seasonal thermocline. As the strength of the stratification of the DWL increases, so does the shear caused by the tunneling internal waves. The analysis demonstrates that internal waves can generate strong enough shear to cause a shear-induced instability, and are a plausible source of the observed enhanced TKE dissipation.

Vertically-varying horizontal transport across the upper ocean occurs because a diurnal current exists within the DWL, but not in the unstratified remnant mixed layer below. Therefore, when a DWL is present, the water within DWL is horizontally transported a different distance than the water below. Coupled with nocturnal convection that mixes the DWL with the unstratified layer at night, this cycle is a mechanism for submesoscale $(1-10 \mathrm{~km})$ lateral diffusion across the upper ocean. Estimates of a horizontal diffusion coefficient are similar in magnitude to current estimates of submesoscale diffusion based on observations, and are likely an important source of horizontal diffusion in the upper ocean.
\end{abstract}

Thesis Supervisor: J. Thomas Farrar

Title: Associate Scientist, Woods Hole Oceanographic Institution

Thesis Supervisor: Carol Anne Clayson

Title: Senior Scientist, Woods Hole Oceanographic Institution 
My thesis and graduate school taught me an important life lesson -

Even on the seemingly calmest of days, turbulence can exist just below the surface.

Things are rarely as simple as they might appear. 


\section{Acknowledgments}

I cannot say that the simple route or going with the flow has ever been my modus operandi. My graduate career started at Florida State University through happenstance; I was being forced to graduate early and decided to stay and a get a Master's degree. I was introduced to Dr. Carol Anne Clayson, who became my advisor.

The rest is quite a long story, but here I am 8 years later finishing my Ph.D. and Carol Anne is still my advisor. I will never be able to truly express all she has done for me over the past eight years at Florida State University and in the Joint Program. She taught the importance of always having a sense of humor and even taught me what snow looks like from an airplane.

There are not words that cannot express my gratitude nor admiration of Tom Farrar. Tom provided the guidance and support I needed to finish. I most certainly would not have a completed thesis if it were not for him. I moved to Washington, DC in the midst of finishing the last chapter of my thesis to start a John A. Knauss Sea Grant Fellowship. Tom consistently went above and beyond what any graduate student could reasonably ask of their advisor.

Ken Brink, John Marshall, and Glenn Flierl have been an example of a what a graduate student could hope for in a committee. They were a source of constant support, believing in me, supporting me, and pushing me to become the best scientists I could.

Although he only served in an official advisor capacity for my first summer, Steve Jayne has been there as a mentor throughout my tenure in the Joint Program. He has been a sounding board and someone to help me process the trials and tribulations of life.

Brent Roberts sat next to me my first three years of graduate school at Florida State University, and was a source of constant support and coding guidance. I am the Diet Coke to his genius. Doug Westphal and the folks at the Naval Research Lab in Monterey, California including Ellen Ramirez were an important part of my graduate career, and I am grateful for the opportunities the NREIP internship opened for me.

I would also like to thank the crew and scientific staff aboard the Fall 2012 (R/V Knorr) and Spring 2013 (R/V Endeavor) SPURS-I cruises, and specifically my graduate student counterparts Julius, Julian, and Jesse. Lou St. Laurent's leadership, advice, and expertise on turbulence instrumentation made my thesis possible. 
There are an incredible number of folks who provided guidance and support along my curvlinear path in the Joint Program including Meg Tivey, Jim Yoder, Julia Westwater, Sarah Xander, Kathy Ponti, and Jason McKnight. All of the amazing folks I met through the Science Policy Initiative, MIT DC Office, and MIT Hillel taught me the importance of the education you receive outside of the lab, office, or classroom.

I would be remiss if I did not thank the incredible physical oceanography students of the Joint Program including Jörn, Izi, Becca, Melissa, Dan, Deepak, Sophie, Jay, and Elise. Graduate school was challenging, but I made it because of the endless support of my friends and awesome cohort, including LJ, Carly, Jason and Kalina, Dave and Rachel, Melissa and John, Leah, Eric, Ben, Britta, Liz, Sarah (and the rest of the Lanes Crew), and Emily and Adam. To my friends near and far, thank you.

The final stretch can be tough, and adding an additional full-time responsibility during the final months was downright challenging. Thank you to the Markey office and my fellow "Knaussers," especially Erica, Anna, and Pat and Ali, for getting me through the final stretch.

To my best friends, Nicole and Sam, for putting up with me for the last eight years of graduate school, I cannot thank you enough for your love and support. I have the most incredible family. My mother is my biggest supporter and fan. My father provided almost daily pep talks and advice. Matt and Stef have been with me through grad school supporting me from the first day at Florida State until now. My adorable niece and nephew, Briana and Gabriel, have brought so much joy into all of our lives. The rest of my family, including the chosen ones, thank you.

My time in the Joint Program was supported by the Department of Defense (DoD) through the National Defense Science \& Engineering Graduate Fellowship (NDSEG) Program and the National Science Foundation under Grant No. OCE-1129646. The collection and analysis of data from the SPURS-I central mooring were supported under National Aeronautics and Space Administration (NASA) Grant No. NNX11AE84G and NNX14AH38G. 


\section{Contents}

1 Introduction $\quad 21$

1.1 Background and Motivation . . . . . . . . . . . . . . . . 22

1.2 Past Work and Present State of Knowledge . . . . . . . . . . . . . . . . 26

1.3 Dissertation Objectives . . . . . . . . . . . . . . . . . 31

1.4 Dissertation Outline . . . . . . . . . . . . . . . . . 31

2 Potential mechanisms for observed enhanced turbulent kinetic energy dissipation in the daytime upper ocean during SPURS-I 35

2.1 Introduction . . . . . . . . . . . . . . . . . . 35

2.2 Observations and cruise details . . . . . . . . . . . . . . . . . 39

2.2.1 SPURS-I Mooring . . . . . . . . . . . . . . . . . 40

2.2.2 Slocum Gliders and Microstructure . . . . . . . . . . . . . . . . . . 40

2.3 The diurnal cycle of TKE dissipation . . . . . . . . . . . . . . . . . . 41

2.4 Canonical 1D view of surface forced mechanisms . . . . . . . . . . . 46

2.4.1 Modeling the near-surface ocean . . . . . . . . . . . . . . . 47

2.4 .2 Monin-Obukhov Similarity Theory . . . . . . . . . . . . . . . . . 54

2.5 Diurnal warm layer shear . . . . . . . . . . . . . . . . . . . 59

2.5.1 Self-regulating diurnal thermocline theory . . . . . . . . . . . . 61

2.5.2 Statistics of shear and stability . . . . . . . . . . . . . 65

2.5.3 Energy of the mean current . . . . . . . . . . . . . . 68

2.6 Summary and Discussion . . . . . . . . . . . . . . . . . . . . . . . . . . . . . . . . . 72

2.6.1 Summary . . . . . . . . . . . . . . . . . . . 72

2.6 .2 Discussion . . . . . . . . . . . . . . . . . . . . . . . 73

3 Near-surface horizontal velocities induced by deep internal waves $\quad \mathbf{7 5}$

3.1 Introduction . . . . . . . . . . . . . . . . . . . . 75

3.2 Data . . . . . . . . . . . . . . . . . . . . 78

3.2.1 SPURS-I Central Mooring . . . . . . . . . . . . . . . . . 78

3.2 .2 Slocum and Wave Gliders . . . . . . . . . . . . . . . . . . . 79

3.2 .3 Isopycnal Displacement . . . . . . . . . . . . . . . . . . . 80

3.3 Observations of Stratification and TKE dissipation . . . . . . . . . . . . . . . 80

3.4 Mixed Layer induced velocities . . . . . . . . . . . . . . . . 88

3.4.1 Observational evidence of D78 mechanism . . . . . . . . . . . . . . 93

3.5 Inclusion of a diurnal warm layer . . . . . . . . . . . . . . . . . 96

3.5.1 The diurnal warm layer as a waveguide . . . . . . . . . . . . . . 102

3.5.2 Potential turbulence generation mechanism . . . . . . . . . . . . . 103

3.5.3 Implications for mixing . . . . . . . . . . . . . . . 106 
3.6 Summary and Conclusion _. . . . . . . . . . . . . . 106

4 Submesoscale lateral mixing by diurnal warm layer shear 111

4.1 Introduction . . . . . . . . . . . . . . . . . . . 111

4.2 Data . . . . . . . . . . . . . . . . . . . . . . . . . . . . . . . . . . .

4.3 Near-surface freshening . . . . . . . . . . . . . . . . . . . . . . 115

4.3.1 Surface Forcing . . . . . . . . . . . . . . . . . . . . . . . . . 118

4.3.2 Near-surface advection . . . . . . . . . . . . . . . . . . . . . . . . . 119

4.3 .3 One-Dimensional Modeling . . . . . . . . . . . . . . . . . 121

4.3.4 Motivation of the mixing mechanism . . . . . . . . . . . . . . 126

4.4 Simple model of mixing . . . . . . . . . . . . . . . . 126

4.5 Estimate of mixing and advection . . . . . . . . . . . . . . . . 133

4.5.1 Estimate of effective diffusivity for observed cases . . . . . . . . . . . . 133

4.5.2 Estimate of advection . . . . . . . . . . . . . . . . . 134

4.5.3 Advective effect on diurnal warming and heat flux . . . . . . . . 136

4.6 Summary \& Discussion . . . . . . . . . . . . . . . . . . . . 137

5 Conclusion $\quad 141$

5.1 Contributions . . . . . . . . . . . . . . . . . . . . 141

5.2 Implications . . . . . . . . . . . . . . . . . . . . . . . 144

5.3 Future Work . . . . . . . . . . . . . . . . . . . . . 146

5.3.1 Langmuir turbulence and surface gravity waves . . . . . . . . . . . . 147

5.3.2 Penetrative solar radiation, and biophysical-chemical interaction . . . 148

5.3.3 Modeling the ocean boundary layer . . . . . . . . . . . . . . . . . 149

\section{A Derivation of turbulent kinetic energy dissipation in Price-Weller-Pinkel} model $\quad 151$

A.1 Derivation of implied MKE \& TKE in PWP . . . . . . . . . . . . . . 152

A.2 Estimation of TKE Dissipation . . . . . . . . . . . . . . 155

B Derivation of Internal Wave induced Horizontal Velocities $\quad 157$

B.1 Derivation with a diurnal warm layer . . . . . . . . . . . . . 158

B.1.1 Surface condition . . . . . . . . . . . . . . . . . . . . 159

B.1.2 Base of diurnal warm layer . . . . . . . . . . . . . . . . . . 159

B.1.3 Seasonal Thermocline . . . . . . . . . . . . . . . . . . 160

B.1.4 Horizontal kinetic energy of interior . . . . . . . . . . . 160

B.1.5 Mean horizontal kinetic energy of DWL . . . . . . . . . . . . . . . . 162

B.1.6 Relationship of DWL and interior KE . . . . . . . . . . . . . . 163

B.1.7 Internal wave induced Richardson number instability . . . . . . . . . 163 


\section{List of Figures}

1-1 Location of the SPURS-I field campaign. The climatological sea surface salinity from the Aquarius satellite mission is contoured in color (Melnichenko et al., 2014). The field campaign took place at the salinity maximum of the North Atlantic Ocean, shown by the black box. . . . . . . . . . . . . . . . . . 22

1-2 Generalized effect of turbulent and radiative fluxes and wind on the ocean. Illustration by Eric S. Taylor, WHOI Graphics. . . . . . . . . . . . . . . . . . 23

1-3 The daily cycle of the upper ocean, including a DWL. During the daytime the upper ocean is heated by the sun creating a DWL or diurnal thermocline. At night the near-surface ocean cools causing convection, mixing the DWL deeper in to the mixed layer, eventually creating a well-mixed layer. Illustration by Eric S. Taylor, WHOI Graphics. . . . . . . . . . . . . . . . . . . . 25

2-1 Location of the SPURS-I field campaign site (top), and density of glider profiles in the area shaded by color in $2.5^{\prime} \times 2.5^{\prime}$ bins near the SPURS-I mooring (black dot with black watch circle) for the deployment $(9 / 15 / 2012$ $10 / 5 / 2012)$ and midterm $(3 / 25 / 2013-4 / 6 / 2013)$ cruises. . . . . . . . . . . 39

2-2 The meteorological conditions at the SPURS mooring $(a, b)$ with the net surface heat flux in red, wind speed in black, and times of active precipitation in light blue. Sea surface temperature $(c, d)$, turbulent kinetic energy dissipation $(\mathrm{e}, \mathrm{f})$, and dissipation of thermal variance $(\mathrm{g}, \mathrm{h})$ from the gliders during the deployment (left-side, 9/15/2012 - 10/5/2012) and midterm (right-side, $3 / 25 / 2013-4 / 6 / 2013)$ cruises. . . . . . . . . . . . . . . . . . . 43

2-3 Average profile of TKE dissipation and dissipation of thermal variance (black line) with standard deviation (dark grey) during the deployment cruise from the deep VMP. Insets of a histogram of all observations for all depths are plotted as well. . . . . . . . . . . . . . . . . . . . . 44

2-4 Hourly averages of the average TKE dissipation of the upper 30 meters of the ocean during (a) wind speed greater than $5 \mathrm{~m} / \mathrm{s}$, and (b) less than $5 \mathrm{~m} / \mathrm{s}$. The black circles indicate the mean, and the black lines indicate the $95 \%$ confidence limits, estimated by bootstrapping. The vertical red dashed lines indicate when the average net surface heat flux changes sign. The number of profiles used in each hourly average in (a) and (b) are shown in (c) and (d), respectively. 
2-5 Composite average TKE dissipation shown by color shading binned by depth and buoyancy frequency (cph) for (a) nighttime light wind conditions $\left(Q_{\text {net }}<\right.$ 0 , wspd $<5 \mathrm{~m} / \mathrm{s}$ ), (b) daytime light wind conditions ( $\mathrm{Q}_{n e t}>0$, wspd $<5$ $\mathrm{m} / \mathrm{s}$ ), and (c) windy conditions (wspd $>5 \mathrm{~m} / \mathrm{s}$ ). The black lines indicate the average buoyancy frequency profile for each condition. The red contour shows where the ocean warms, on average, more than $0.25^{\circ} \mathrm{C}$ from the value at sunrise at each depth, based on the light wind daytime composite, and is plotted on all three for reference. . . . . . . . . . . . . . . . . . 46

2-6 The modeled $(\mathrm{a}, \mathrm{b})$ temperature, $(\mathrm{c}, \mathrm{d})$ salinity, and $(\mathrm{e}, \mathrm{f})$ current speed from the Kantha-Clayson (KC, blue lines) and Price-Weller-Pinkel (PWP, red lines) models with observations from the mooring (black line). The shallowest observation is compared to the model estimate at that depth, which is $0.75 \mathrm{~m}$ for temperature and salinity, and $3.5 \mathrm{~m}$ for currents (referenced to the current at $50 \mathrm{~m})$. The meteorological conditions at the SPURS mooring $(\mathrm{g}, \mathrm{h})$ with the net surface heat flux in red, wind speed in black, and times of active precipitation in light blue during the deployment (left-side, 9/15/2012 - 10/5/2012) and midterm (right-side, $3 / 25 / 2013-4 / 6 / 2013$ ) cruises. . . . . . . . . . . 49

2-7 Depth-averaged turbulent kinetic energy dissipation over the upper 30 meters from (a,b) Monin-Obukhov Similarity Theory (MOST) , (c,d) the KanthaClayson (KC) model, (e,f) the Price-Weller-Pinkel (PWP) model, and $(\mathrm{g}, \mathrm{h})$ microstructure observations for the deployment (left-side, 9/15/2012 - 10/5/2012) and midterm (right-side, 3/25/2013 - 4/6/2013) cruises. The red dashed line and filled curve on all plots is scaled incoming solar radiation to show times when the sun is up. . . . . . . . . . . . . . . . . . . . 5

2-8 Observations (left), Kantha-Clayson model results (middle), and Price-WellerPrinkel model results (right) of $(\mathrm{a}, \mathrm{b}, \mathrm{c})$ temperature, $(\mathrm{d}, \mathrm{e}, \mathrm{f})$ buoyancy frequency, $(\mathrm{g}, \mathrm{h}, \mathrm{i})$ shear squared, and $(\mathrm{j}, \mathrm{k}, \mathrm{l})$ reduced shear $\left(S^{2}-4 N^{2}>0\right.$ indicates flow with a $\mathrm{Ri}<0.25)$ from the deployment cruise time period in which the glider was profiler near the central mooring $(9 / 22 / 2012-10 / 5 / 2012)$. The white line indicates 4 meters, which is the minimum depth of the shear observations. Note the high shear below 4 meters that is not simulated by the models, as the shear during the daytime is confined above the white line in the models.

2-9 Scatter plot of the buoyancy Reynolds number $\left(\mathrm{Re}_{\mathrm{b}}\right)$ calculated from the glider dissipation and a buoyancy Reynolds number for MOST ( $\left.\operatorname{Re}_{b-M O}\right)$ under (a) stabilizing surface forcing and (b) destabilizing surface forcing (b) averaged over the upper 10 meters of the ocean. The color indicates the absolute value of Obukhov length, and it is positive in (a) for stable forcing, and negative in (b) for destabilizing forcing. The dashed line is the best fit for all of the data in each regime. The dotted line is the best fit for data with $\left|\mathrm{L}_{o}\right|>3$, corresponding to the largest color bar interval and roughly half the depth over which the data is averaged. Note the drop off at small Obukhov lengths for destabilizing surface forcing and large scatter for stabilizing surface

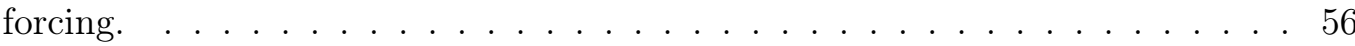


2-10 Similar to Fig. 2-5, but composite of average shear squared shown by color shading binned by depth and buoyancy frequency (cph) for (a) nighttime light wind conditions ( $\mathrm{Q}_{n e t}<0$, wspd $<5 \mathrm{~m} / \mathrm{s}$ ), (b) daytime light wind conditions ( $Q_{n e t}>0$, wspd $<5 \mathrm{~m} / \mathrm{s}$ ), and (c) windy conditions (wspd $>5$ $\mathrm{m} / \mathrm{s}$ ). The black lines indicate the average buoyancy frequency profile for each condition. The red contour shows where the ocean warms, on average, more than $0.25^{\circ} \mathrm{C}$ from the value at sunrise at each depth, based on the light wind daytime composite, and is plotted on all three for reference. . . . . . . .

2-11 Similar to Fig. 2-5 and 2-10, but composite of average reduced shear $\left(\mathrm{S}^{2}\right.$ $4 \mathrm{~N}^{2}$, where values greater than 0 indicate $\mathrm{Ri}<0.25$ corresponding to unstable Kelvin-Helmholtz conditions) shown by color shading binned by depth and buoyancy frequency (cph) for (a) nighttime light wind conditions $\left(\mathrm{Q}_{\text {net }}<0\right.$, wspd $<5 \mathrm{~m} / \mathrm{s}$ ), (b) daytime light wind conditions ( $\mathrm{Q}_{\text {net }}>0$, wspd $<5$ $\mathrm{m} / \mathrm{s}$ ), and (c) windy conditions (wspd $>5 \mathrm{~m} / \mathrm{s}$ ). The black lines indicate the average buoyancy frequency profile for each condition. The red contour shows where the ocean warms, on average, more than $0.25^{\circ} \mathrm{C}$ from the value at sunrise at each depth, based on the light wind daytime composite, and is plotted on all three for reference. . . . . . . . . . . . . . . . . 62

2-12 Diurnal phase composite of 18 days with diurnal warming and no measurable precipitation during the SPURS-I deployment and midterm cruises. (a) Net surface heat flux average (red line) and the number of glider profiles in each hourly average (black line) for the composited days. Composite diurnal phase average of (b) four times the buoyancy frequency from the mooring, (c) shear squared from the mooring, (d) calculated reduced shear by matching observations of buoyancy frequency nearest the observations of shear in time, (e) TKE dissipation from the gliders, and (f) percent of time the calculated reduced shear is greater than zero, in local time. Vertical red dashed lines indicate time periods of positive and negative net surface heat flux, and the black contour is the zero line of reduced shear (equivalent to a $\mathrm{Ri}$ of 0.25 ). . .

2-13 Empirical probability distribution functions of TKE dissipation and reduced shear, respectively, for depth bins of $(\mathrm{a}, \mathrm{b})$ 4-10 m (the shallowest observations limited the top of the near-surface bin), (c,d) 10-20 m, and (e,f) 20-30 m for nighttime light wind conditions (blue, $\mathrm{Q}_{\text {net }}<0$, wspd $<5 \mathrm{~m} / \mathrm{s}$ ), daytime light wind conditions (red, $\mathrm{Q}_{n e t}>0$, wspd $<5 \mathrm{~m} / \mathrm{s}$ ), and windy conditions (black, wspd $>5 \mathrm{~m} / \mathrm{s}$ ). Each histogram is normalized such that the area under the curve is unity. The statistics of the distributions are presented in Tables 2.2 and $2.3 \ldots \ldots \ldots \ldots$ 
2-14 The upper 10 meter (a,b) integrated kinetic energy from the ADCP (Eq. 2.8, black line assumes slab layer above 3.5 meters and red line assumes a linear shear with a surface current twice as fast as 3.5 meters) and integrated TKE dissipation from the gliders (blue bars, Eq. 2.9) and (c,d) dissipation time-scale (Eq. 2.10). (e,f) The required speed of a slab layer for wind work to explain the observed TKE dissipation of the upper 10 meters (black line, Eq. 2.12) and mean current of the upper 10 meters (blue line). The meteorological conditions at the SPURS mooring $(\mathrm{g}, \mathrm{h})$ with the net surface heat flux in red, wind speed in black, and times of active precipitation in light blue during the deployment (left-side, 9/15/2012 - 10/5/2012) and midterm (right-side, 3/25/2013 - 4/6/2013) cruises. Note the sharp decrease in the dissipation time-scale in (c) and (d) and unrealistic surface current required in (e) and (f) during daytime conditions of diurnal warming and observed enhanced dissipation. . . . . . . . . . . . . . . . .

3-1 The meteorological conditions at the SPURS mooring $(a, b)$ with the net surface heat flux in red, wind speed in black, and times of active precipitation in light blue. Sea surface temperature $(c, d)$, buoyancy frequency in cycles per hour $(\mathrm{e}, \mathrm{f})$, and turbulent kinetic energy dissipation $(\mathrm{g}, \mathrm{h})$ from the Slocum gliders during the deployment (left-side, 9/15/2012 - 10/5/2012) and midterm (right-side, $3 / 25 / 2013-4 / 6 / 2013$ ) cruises. . . . . . . . . . . . . . . . . . 81

3-2 The meteorological conditions at the SPURS mooring (a,b) with the net surface heat flux in red, wind speed in black, and times of active precipitation in light blue. Depth averaged buoyancy frequency (black line) and turbulent kinetic energy dissipation (red bars) for 0-5 meter bin (c,d), 10-15 meter bin $(\mathrm{e}, \mathrm{f})$ and 20-25 meter bin $(\mathrm{g}, \mathrm{h})$ from the gliders during the deployment (leftside, 9/15/2012 - 10/5/2012) and midterm (right-side, 3/25/2013 - 4/6/2013) cruises. . . . . . . . . . . . . . . . . . . 82

3-3 Turbulent kinetic energy dissipation versus buoyancy frequency squared, averaged over the daylight hours of a day over the upper 10 meters from the Slocum gliders during SPURS-I deployment (9/15/2012 - 10/5/2012) and midterm $(3 / 25 / 2013-4 / 6 / 2013)$ cruises. The color represents the windspeed from the mooring averaged over the same time period. The black line is the least squares best fit line. . . . . . . . . . . . . . . . . 83

3-4 Path of the three Wave Gliders deployed during the SPURS-I field campaign for the time period of September $28-29,2012 \ldots \ldots \ldots$. . . . . . 84

3-5 Observations from the three Wave Gliders of (a,c,e) temperature and (b,d,e) salinity (black lines) and corresponding gradients (blue lines) at a depth of $0.3 \mathrm{~m}$ for the time period of September 28 - 29, 2012. The shaded regions indicate when the the set surface heat flux is heating the ocean. The color choice identifies the Wave Glider, consistent with Fig. 3-4. The vertical dash line indicates turning points of the gliders. The $\mathrm{x}$-axis is selected as distance to highlight the horizontal gradients observed by the gliders. . . . . . . . . . 85 
3-6 Turner angle diagram modified after Ruddick (1983) and Schmitt (2011). The vertical axis is the vertical density gradient due to temperature, and the horizontal axis is the vertical density gradient due to salinity. The Turner angle $(\mathrm{Tu})$ is defined clockwise from where marked. "Stable or "unstable" references the static stability of the vertical density gradient, and the temperature and salinity identifiers denote the water mass atop. For example, "warmer, saltier" means that warmer and saltier water is atop relatively cooler and fresher water. . . . . . . . . . . . . . . . . . . 87

3-7 Scaled (a) vertical ( 0.3 to $6 \mathrm{~m}$ ) and (b) horizontal temperature gradients (from the $0.3 \mathrm{~m}$ CTD) from the Wave Gliders for the time period of September 28 - 29, 2012. The gradients are scaled by the thermal expansion coefficient and saline contraction coefficient, respectively, such that temperature and salinity are shown as comparable density changes. The color represents the Turner angle, and axis are those explained by Fig. 3-6. The blue and red dotted line represent the best fit for the freshwater lens and diurnal warming regimes for the days, respectively. . . . . . . . . . . . . . . . 88

3-8 Simplified upper ocean density structure for (a) a well-mixed layer with seasonal thermocline treated as a density jump (This is Fig. 1 adapted from D'Asaro, 1978) and (b) a stratified diurnal warm layer above a so-called remnant mixed layer. . . . . . . . . . . . . . . . . . . . . . . . . . . 89

3-9 Wavenumber-frequency diagram of the horizontal kinetic energy (HKE) ratio of the mixed layer to the stratified interior as defined in Fig. 3-8 for (a) the case shown with a non-dimensional density jump (given as $\Delta \rho / \rho_{0}$ ) of 0.001 , (b) the case without a density jump at the seasonal thermocline, and (c) the difference between the case with the density jump in (a) and without (b) to show the effect of the idealized seasonal thermocline. . . . . . . . . . . . . 92

3-10 Horizontal kinetic energy ratio of the mixed layer to the stratified interior as a function of frequency, numerically integrated over wavelength, using the Garrett and Munk spectrum for IWs in the stratified interior. The horizontal $\mathrm{KE}$ ratio as a function of wavenumber and frequency is shown by Eq. 3.7. A $50 \mathrm{~m}$ deep mixed layer is used with a non-dimensional density jump (given as $\left.\Delta \rho / \rho_{0}\right)$ of $0.001 \ldots \ldots \ldots \ldots \ldots \ldots \ldots \ldots \ldots \ldots$

3-11 Average potential temperature profile from September 15 - October 15, 2012 from the SPURS-I mooring center at $25^{\circ} \mathrm{N}, 38^{\circ} \mathrm{W}$. The grey fill indicates the standard deviation of the average profile. The three x's indicate the depths of the deep temperature observations. Above 400 meters the mooring is wellinstrumented. . . . . . . . . . . . . . . . . . . 95

3-12 The (a) horizontal kinetic energy (HKE) spectral density and (b) displacement spectra for a range of depths from the SPURS-I central mooring from September 15 - October 15, 2012. The starred depths indicate deep VMCMs instead of ADCP measurements, which are every minute and hour, respectively. The shallowest displacement spectra is at the depth of the shallowest isopycnal that existed for the entire month period. The depth denoted for displacement is the average depth of the isopycnal over the one month time period. The red circle and vertical line shows the extent of the $95 \%$ confidence level on the spectral estimates. The vertical dashed lines indicate important frequencies. Note the peak in displacement spectra at the buoyancy frequency of the deep ocean, $N_{0} \ldots \ldots \ldots \ldots \ldots$. . . . . . . . . . . . 97 
3-13 Daytime temperature variance (in log scale) at the $0.75 \mathrm{~m}$ CTD estimated over a day with a 4-hour high pass filter to remove the large diurnal amplitudes versus daily mean wind speed for the entire SPURS-I record. The The color indicates the magnitude of warming over the day. The days with observed high TKE dissipation are indicated as stars. As wind speed decreases, daytime temperature variances increases. . . . . . . . . . . . . . . . .

3-14 Shear squared calculated from the simple model with a diurnal warm layer with an $\mathrm{N}_{\mathrm{d}}$ of $(\mathrm{a}, \mathrm{d}, \mathrm{g}) 25 \mathrm{cph},(\mathrm{b}, \mathrm{e}, \mathrm{h}) 10 \mathrm{cph}$, and (c,f,i) $1 \mathrm{cph}$. For all panels, a density jump of $1 \mathrm{~kg} / \mathrm{m}^{3}$ is used at a seasonal thermocline. For the first column, a seasonal thermocline depth of $50 \mathrm{~m}$ with a diurnal warm layer depth of $5 \mathrm{~m}$ is used. For the second column, the diurnal warm layer depth is doubled to $10 \mathrm{~m}$. For the third column, the seasonal thermocline depth is increased to $300 \mathrm{~m}$. Note Fig. 3-9 is similarly shows results in a wavenumberfrequency space however, here a log-log scale is use to highlight low frequencies and wavenumbers. . . . . . . . . . . . . . . . . . . 101

3-15 The (a) mean-square shear (over all wavenumber and frequency space) and (b) the probability of shear causing a $\mathrm{Ri}<0.25$ assuming zero mean shear and using the mean-square shear as the variance of a Gaussian probability function. This setup includes a density jump of $1 \mathrm{~kg} / \mathrm{m}^{3}$ used at a seasonal thermocline depth of $50 \mathrm{~m}$ with a diurnal warm layer depth of $5 \mathrm{~m}$ (the first column of Fig. 3-14). The dashed line is (a) is 4 times $\mathrm{N}^{2}$ to show the value at which $\mathrm{Ri}=0.25 \ldots \ldots \ldots \ldots$. . . . . . . . . . . . . . . . . . . . . . .

3-16 Buoyancy frequency of observed days with during SPURS-I cruises dailyaveraged over the upper 10 meters (only during daylight hours) from the Slocum gliders versus the fractional area of wavenumber-frequency space estimated from the simple IW model. The color indicates the daily-average TKE dissipation from the gliders over 10 meters. The black line is the least squares best fit line. . . . . . . . . . . . . . . . . . . 105

4-1 Two days of observed near-surface freshening during the SPURS-I deployment cruise on September 28 and 29, 2012: a/e) net surface heat flux; b/f) zonal and meridional wind speed; $\mathrm{c} / \mathrm{g}$ ) temperature observations from the mooring; $\mathrm{d} / \mathrm{h}$ ) salinity observations from the mooring. The grey shading indicates the time period of the advective event. . . . . . . . . . . . . . . 116

4-2 Another example day of observed near-surface freshening on April 29, 2013: a) net surface heat flux; b) zonal and meridional wind speed; c) temperature observations from the mooring d) salinity observations from the mooring. The grey shading indicates the time period of the advective event. The day was not during a cruise . . . . . . . . . . . . . . . . . . 117

4-3 Two types of advection that can cause the observed near-surface freshening during the daytime. Top: Near-surface advection of a lateral gradient. This would be the case with a diurnal warm layer current. Bottom: Mean advection of a strong vertical gradient. . . . . . . . . . . . . . . . . . 120 
4-4 Normalized histogram of observed salinity difference between the $0.3 \mathrm{~m}$ and $6.0 \mathrm{~m} \mathrm{CTD}\left(\Delta S_{z}\right)$ of the Wave Gliders over \pm 1 day for a) September 28, 2012, b) September 29, 2012, and c) April 29, 2013. The black dotted line is the empirical cumulative distribution function. The normalization factor is included as the maximum number of observations in a bin. The histogram is cut off at $10^{-2} \mathrm{psu}$ as that is a conservative estimate of the accuracy of the CTDs (the first bin includes all vertical differences less than $10^{-2} \mathrm{psu}$ ). . . . . 121

4-5 Observations of salinity from the SPURS-I study area $\left(1^{\circ} \times 1^{\circ}\right.$ box $)$ during \pm 1 day from the observed near-surface freshening on a) September 28, 2012, b) September 29, 2012 (two days shown in Fig. 4-1), and c) April 29, 2013 (day shown in Fig. 4-2) from the Slocum gliders (0.5-1 m depth, seemingly randomly dispersed dots), Wave Gliders $(0.35 \mathrm{~m})$, and thermosalinograph from the ship (only in a and b, nominally $5 \mathrm{~m}$ ). The starting point for a given time period of the path of each Wave Glider is denoted by a colored star and ship denoted by the triangle. The black $\mathrm{X}$ is the location of the SPURS-I mooring. . . . . . . . . . . . . . . . . . . . . . . 122

4-6 Modeled and observed temperature profiles every 2 hours for the three days with observed near-surface freshening, September 28 and 29, 2012 and April 28, 2013 from a/b/c) Kantha-Clayson, d/e/f) Price-Weller-Pinkel, and g/h/i) the SPURS-I central mooring. The color indicates the time of the day. Note the different temperature scale for each day. . . . . . . . . . . . . . . . . . . . 124

4-7 Modeled current speed profiles every 2 hours for the three days with observed near-surface freshening, September 28 and 29, 2012 and April 28, 2013 from a/b/c) Kantha-Clayson and d/e/f) Price-Weller-Pinkel models. The color indicates the time of the day. . . . . . . . . . . . . . . . . . . 125

4-8 Schematic of the simplified representation of the mixing mechanism. The night before a front (delta gradient) exists over a well-mixed layer of depth, $H$. During the daytime diurnal warm layer shear exists at the near-surface due to stabilization from incoming solar radiation over a layer of depth, $\delta$, with a current $u_{o}$ assuming no background current of the mixed layer. The surface layer can travel a distance $\Delta x$ over a diurnal cycle that is $\Delta t$ long. Nocturnal convection ensues when the net surface flux changes sign, cooling the ocean surface. A new salinity $S_{3}$ will be observed in the region where differential advection by the diurnal warm layer shear occurred at the near-surface, which is in between the two observed salinities the night before. . . . . . . . . . . 127

4-9 The number of times, $n$, the cycle of advection then mixing would have to occur in order for the salinity in the right box of Fig. 4- 8 to be within $99.9 \%$ of $S_{0}$. The number of cycles is plotted as a function of the non-dimensional salinity change and non-dimensional depth of the diurnal warm layer depth. The black astrisk indicates the number of cycles necessary to erase the observed $\Delta S$ (corresponding so a $\Delta S_{0}$ ) on September 29, 2012 assuming a 20 $\mathrm{m}$ mixed layer and $1 \mathrm{~m}$ diurnal warm layer. The black star indicates the number of cycle necessary to erase a 0.1 psu difference assuming a diurnal warm layer that is $1 / 3$ the depth of the mixed layer (which assumes weaker diurnal warming) . . . . . . . . . . . . . . . . . . . . . . . 129

4-10 Estimated effective horizontal diffusivity from the simple model for a range of diurnal warm layer velocity increases $\left(u_{0}\right)$, for an advective time-scale of 6 hours using Eq. 4.6. . . . . . . . . . . . . . . . . . . . . . . 131 
4-11 Normalized stacked histogram of diurnal sea surface temperature (dSST, maximum during the daytime minus minimum at night) from the Clayson diurnal warming parameterization of Bogdanoff et al. (In Preparation) over the 10 year periods from January 1, 1998 - December 31, 2007 (grey) and observed dSST from the over the 383 day long deployment of SPURS mooring (black). The black dotted lines are empirical cumulative distribution function. The normalization factor is included as the maximum number of observations in a bin. . . . . . . . . . . . . . . . . . . . . . . . 132

4-12 Normalized stacked histogram of observed lateral salinity gradients over \pm 1 day for a) September 28, 2012, b) September 29, 2012, and c) April 29, 2013 from the shipboard thermosalinograph (when available, black, nominally at $5 \mathrm{~m}$ ) and Wave Gliders (grey, $0.35 \mathrm{~m}$ ). The black dotted line is the empirical cumulative distribution function. The gradients are calculated in the direction of the instrument movement, $m$. The normalization factor is included as the maximum number of observations in a bin. . . . . . . . . . . . . . . . . 135

4-13 Normalized stacked histogram of observed temperature gradients over \pm 1 day for a) September 28, 2012, b) September 29, 2012, and c) April 29, 2013 from the shipboard thermosalinograph (when available, black) and Wave Gliders (grey). The black dotted line is the empirical cumulative distribution function. The gradients are calculated in the direction of the instrument movement, $m$. The normalization factor is included as the maximum number of observations in a bin. . . . . . . . . . . . . . . . . . . . 136

4-14 Global percentage of days with diurnal warming greater than $0.1^{\circ} \mathrm{C}$ from the Clayson diurnal warming parameterization of Bogdanoff et al. (In Preparation) over the 10 year periods from January 1, 1998 - December 31, 2007. . . 138

5-1 Global a) percentage of days with diurnal warming greater than $0.1^{\circ} \mathrm{C}$ from the Clayson diurnal warming parameterization of Bogdanoff et al. (In Preparation) over the 10 year periods from January 1, 1998 - December 31, 2007; b) annual averaged mixed layer depth based on the Holte and Talley (2009) Argo float climatology. . . . . . . . . . . . . . . . . . . . . . . 145 


\section{List of Tables}

2.1 Glider missions during the deployment and midterm cruises, including the instrument type, dates of mission, number of profiles, and mission location description. While all the data is used in the analysis of the diurnal cycle of TKE dissipation, only those deployments near the central mooring are used throughout the rest of the study. . . . . . . . . . . . . . . . . . . 42

2.2 Statistics of the probability distribution of $\log _{10}$ TKE dissipation shown in Fig. 2-13 (a, c, and e) for nighttime light wind conditions $\left(\mathrm{Q}_{n e t}<0\right.$, wspd $<5 \mathrm{~m} / \mathrm{s}$ ), daytime light wind conditions $\left(\mathrm{Q}_{\text {net }}>0\right.$, wspd $<5 \mathrm{~m} / \mathrm{s}$ ), and windy conditions (wspd $>5 \mathrm{~m} / \mathrm{s}$ ) for 10 meter depth bin intervals from the surface to 30 meters. . . . . . . . . . . . . . . . . . . . 66

2.3 Statistics of the probability distribution of $10^{4} \mathrm{x}$ reduced shear shown in Fig. 2-13 (b, d, and f) for nighttime light wind conditions $\left(\mathrm{Q}_{\text {net }}<0\right.$, wspd $<5 \mathrm{~m} / \mathrm{s}$ ), daytime light wind conditions $\left(Q_{n e t}>0\right.$, wspd $<5 \mathrm{~m} / \mathrm{s}$ ), and windy conditions (wspd $>5 \mathrm{~m} / \mathrm{s}$ ) for 10 meter depth bin intervals from the surface to 30 meters. . . . . . . . . . . . . . . . . . . . . . 67

4.1 Near-surface characteristics of days with observed freshening during the daytime, including the daily average wind speed, total rainfall, and diurnal change in sea surface temperature (dSST). $\Delta \mathrm{S}$ and $\Delta \mathrm{S}_{F}$ are the observed and possible change in salinity from the surface fluxes (Eq. 4.2), respectively, over a 0.75 meter surface layer from sunrise until the maximum change in salinity during the daytime (which is time $\Delta t_{\max }$ to the nearest quarter hour). $\Delta t$ is the estimated length of the advective event, rounded to the nearest quarter hour. $\mathbf{P}$ is the total required rain fall in order to explain the near-surface freshening from precipitation. . . . . . . . . . . . . . . . . 118 


\section{Chapter 1}

\section{Introduction}

Many phenomena interacting simultaneously make the near-surface ocean a complex region to characterize. At the air-sea interface, turbulent and long wave radiative fluxes generally act to cool the ocean surface. Incoming solar radiation acts to stabilize the photic zone, or light-touched portion, of the ocean during the daytime. Wind acting on the ocean surface transfers mechanical energy from the atmosphere to the ocean tending to create a well-mixed upper ocean. However, when the winds are not too strong, the incoming solar radiation can cause a stably stratified near-surface layer called the Diurnal Warm Layer (DWL). The depth of the D WL can range from several centimeters to tens of meters (Soloviev and Lukas, 1997; Prytherch et al., 2013). Less regular phenomena, such as rain, can also dramatically change the structure of the upper ocean almost instantaneously.

This dissertation focuses on the daily cycle of the upper ocean, specifically examining DWLs. Although the ocean is sometimes conceptualized as consisting of a mixed layer atop the stratified interior ocean, the DWL can play an important role in changing the physics and dynamics of the upper ocean. Novel observational platforms, such as the Slocum Gliders used in this study, have allowed for detailed observations of the DWL, revealing a turbulent and active layer.

The initial Salinity Processes in the Upper ocean Regional Study, or SPURS-I, field campaign was focused on the climatological surface salinity maximum in the North Atlantic Ocean. Accompanying the campaign were with three month-long cruises to the SPURS-I study region (centered at $25^{\circ} \mathrm{N}, 38^{\circ} \mathrm{W}$, Fig. 1-1, Lindstrom et al., 2015). A follow-on field campaign, SPURS-II, is being conducted in a fresh region of the Pacific Ocean in 2016-2017. 


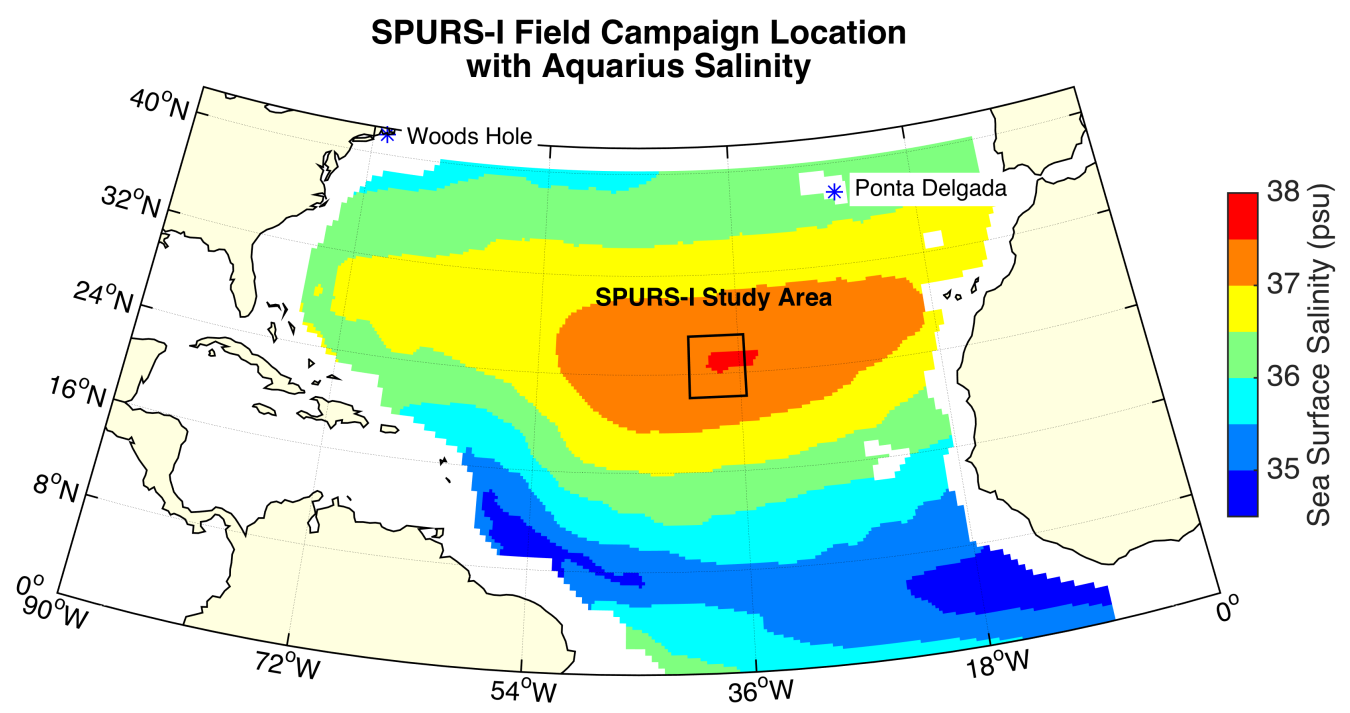

Figure 1-1: Location of the SPURS-I field campaign. The climatological sea surface salinity from the Aquarius satellite mission is contoured in color (Melnichenko et al., 2014). The field campaign took place at the salinity maximum of the North Atlantic Ocean, shown by the black box.

\subsection{Background and Motivation}

The upper 2.5 meters of the ocean contains as much heat capacity per unit area as the entire depth of the atmosphere above (Gill, 1982); therefore, understanding the upper ocean is vital for a complete picture of the energy and water cycle of the Earth's climate system. DWLs change the structure of the upper ocean and directly affect how the ocean and atmosphere interact, due to the interaction of the air and the sea at the free surface interface between the two fluid bodies. At this interface, momentum, moisture, and heat are transferred through wind stress, and turbulent and radiative fluxes (Webster et al., 1996), shown graphically in Fig. 1-2.

Specifically, shortwave radiation from the sun heats the ocean during the daytime. The sensible heat flux typically acts to cool the warmer sea surface, as heat is transferred from the warmer fluid to the cooler one. The latent heat flux transfers energy from the ocean to the atmosphere through evaporation. Wind drives mechanical mixing, vertically stirring the ocean, tending to create a vertically well-mixed upper ocean. When the wind is light and vertical mixing is weak, the near-surface ocean can stratify during the daytime due to 
The sun gives off high-energy shortwave radiation.

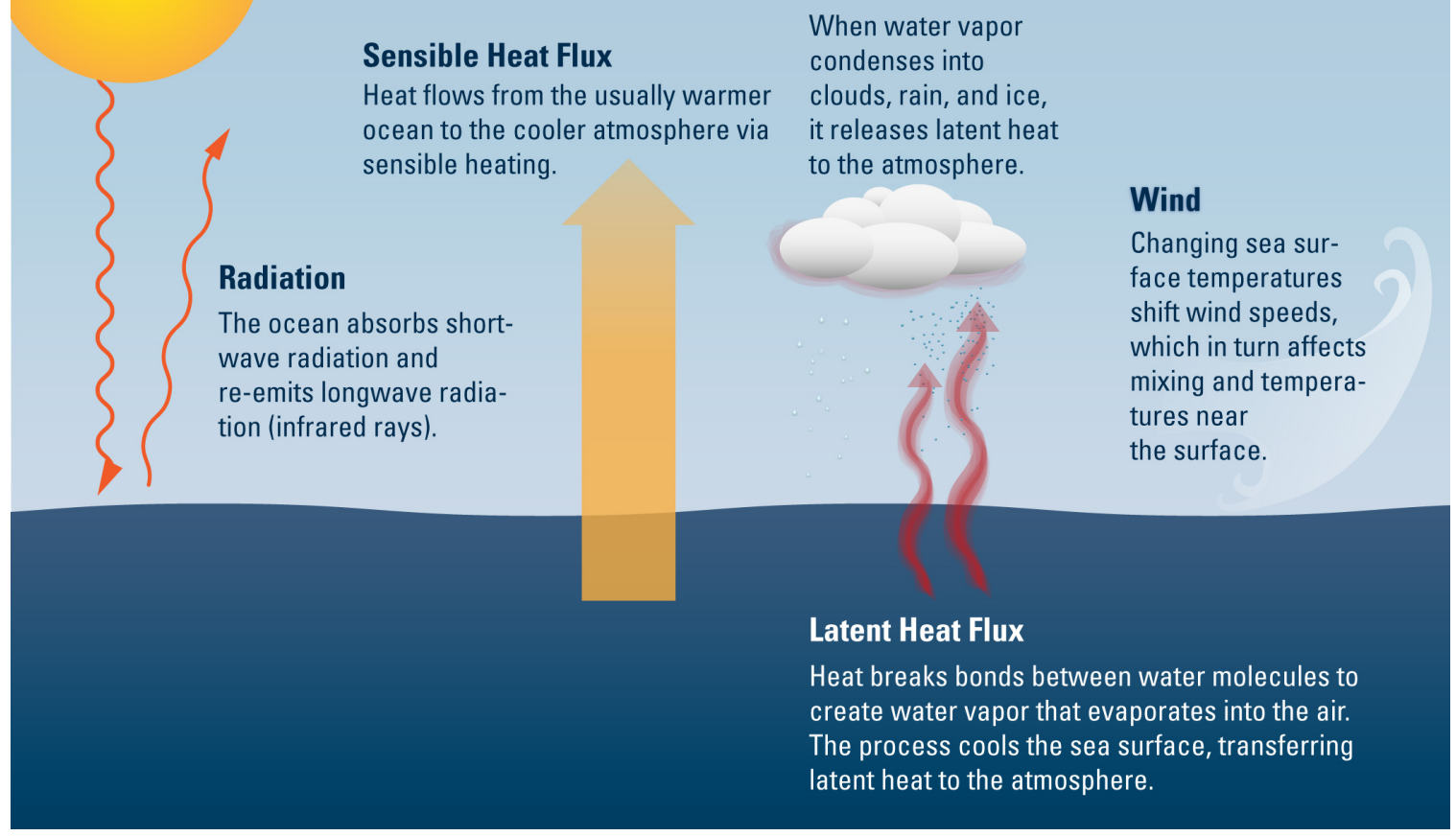

Figure 1-2: Generalized effect of turbulent and radiative fluxes and wind on the ocean. Illustration by Eric S. Taylor, WHOI Graphics.

the shortwave radiation. These stratified layers are DWLs (Soloviev and Lukas, 2006).

Additionally, air-sea gas exchange occurs at this boundary and is modulated by the same processes (Soloviev et al., 2002). The structure of the near-surface layers of the atmosphere and ocean affects the magnitude of the transfer. Many aspects of the structure and processes of the boundary layers have been observed and well studied. However, there are still substantial questions about the relatively thin, stably stratified upper ocean boundary layer, mostly due to a lack of necessary observations. New instrument platforms and techniques provide novel ways to observe and investigate the upper ocean, and more specifically the relatively shallow stable layers (Daniel et al., 2011; Peterson and Fer, 2014). This dissertation explores those layers.

Turbulent fluxes are governed by a number of parameters, with surface temperature being a primary variable (Fairall et al., 1996). In fact, Sea Surface Temperature (SST) is considered the most important variable controlling air-sea energy exchange (Large et al., 1994), and it is thus important to measure it accurately. Due to the inability to directly measure fluxes except with special instrumentation (for example, the direct covariance flux 
system at WHOI; Edson et al., 1998) bulk formulas are used to estimate fluxes. As bulk formulas are based purely on empirical relationships, drag coefficients and formulations continue to be improved, such as in Fairall et al. (2003). These require SSTs as an input, and SST can be the largest source of error in the estimations, especially under low-wind speed conditions (Fairall et al., 1996; Ward, 2006). Under conditions of stable stratification, the ocean skin temperature can be several degrees warmer than a bulk SST $(\sim 1-5 \mathrm{~m})$ observation from a ship or buoy (Donlon et al., 2007). It is the skin SST that is most appropriate for use in turbulent flux calculations (Curry et al., 2004).

Clayson and Bogdanoff (2013) built on previous studies, such as Ward (2006), to explore the importance of using the skin temperature in air-sea flux calculations by parameterizing the diurnal warming magnitude (how much the ocean warms on a daily basis). Errors in the heat fluxes of $10-50 \mathrm{~W} / \mathrm{m}^{2}$ (which can be $100 \%$ error) are possible during low-wind conditions in the daytime with the primary contribution to error being the difference in temperature across the DWL. As the surface heats up, the difference between the air and sea temperatures increases, creating larger fluxes. Diurnal warming is important to consider when calculating air-sea fluxes, as the error with ignoring it can propagate through a broad range of spatial and temporal scales. Remotely sensed SSTs are a prime example of how incorrect treatment of diurnal warming can create significant biases in datasets (Dash et al., 2010).

Detailed temperature, salinity, and turbulence values of DWLs are difficult to obtain given the relatively large changes over a small vertical distance and the transient nature of the layers. In addition, the depth of the DWL can be the same order of magnitude as the height of surface waves further complicating the measurements, including the appropriate depth to use for measurements. Observations of the vertical structure DWLs are generally limited to field projects with measurements of only temperature and conductivity (e.g. CBLASTLOW, Edson et al., 2007). DWLs can be on the order of centimeters to meters thick. As such, most of the geoscience community's knowledge of the stable boundary layer (SBL, a general term for stably stratified layers such as DWLs) comes from meteorological experiments, as the SBL in the atmosphere is orders of magnitude thicker than in the ocean (Poulos et al., 2002).

The upper ocean is often simplified as a well-mixed slab layer atop the stratified ocean. However, that is not always the case (see Fig. 1-3). At night, when the ocean surface 


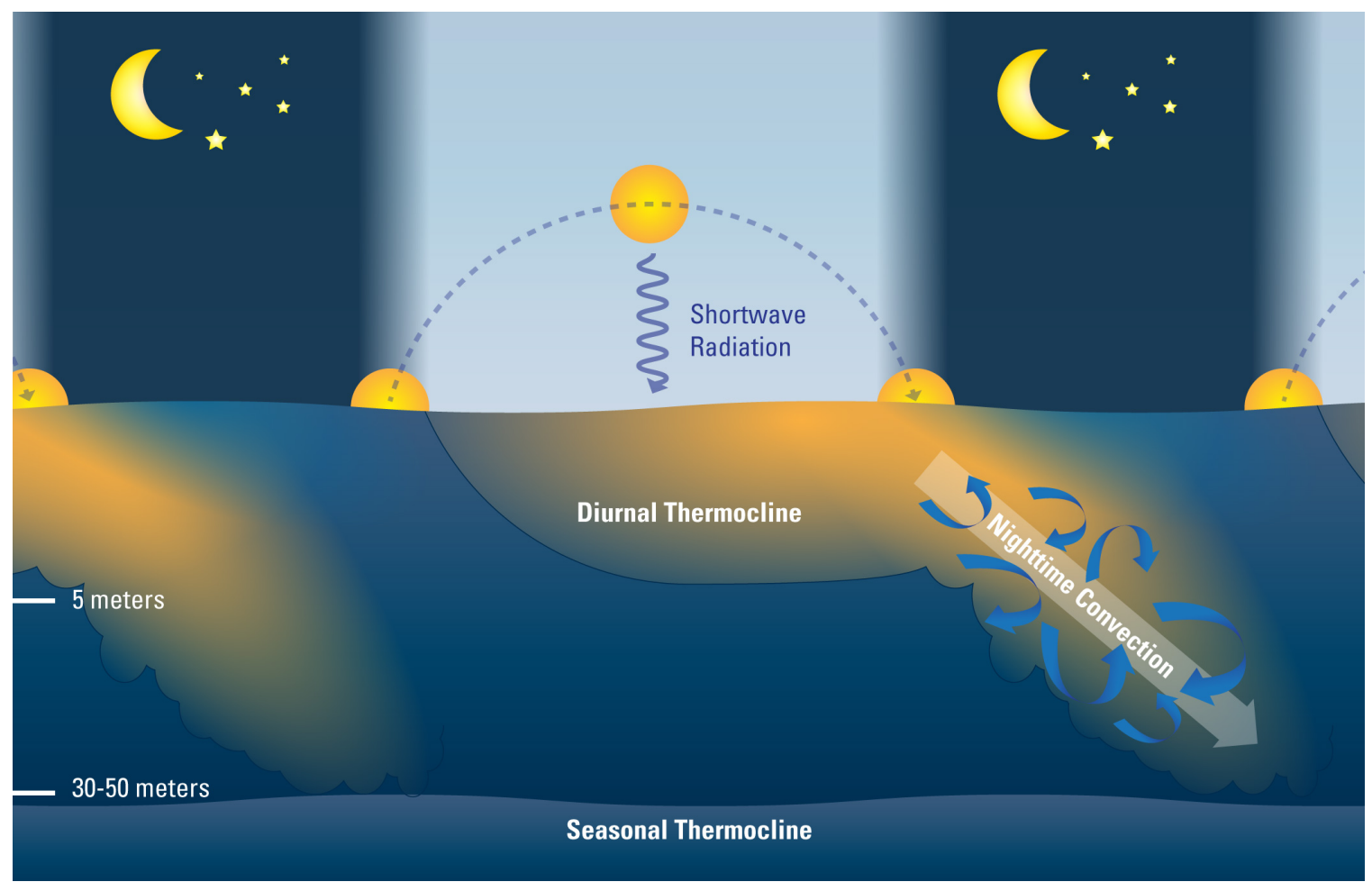

Figure 1-3: The daily cycle of the upper ocean, including a DWL. During the daytime the upper ocean is heated by the sun creating a DWL or diurnal thermocline. At night the near-surface ocean cools causing convection, mixing the DWL deeper in to the mixed layer, eventually creating a well-mixed layer. Illustration by Eric S. Taylor, WHOI Graphics.

cools and convection ensues (Shay and Gregg, 1986) a well-mixed layer can exist. During the daytime stratified DWLs can occur. The DWLs are eventually completely mixed out due to nocturnal convection after the sun sets. It is the convective nighttime layer that is thought to be the most turbulent (Moum et al., 1989). However, Clayson et al. (2016b) found that under diurnal warming conditions large values of turbulent kinetic energy (TKE) dissipation $\left(\epsilon>10^{-5} \mathrm{~W} / \mathrm{kg}\right)$ are observed. These observations are a primary motivation for this dissertation, leading to the question: Why is there observed enhanced TKE dissipation during the daytime?

Additionally, this work is part of the SPURS-I project. A main goal of the SPURS-I project is to understand the oceanic component of the water cycle and how it may change with a changing climate. The salinity of the ocean surface is directly affected by changes in the global water cycle and is a potentially effective way to study the water cycle (e.g. Li et al., 2016). The location of the SPURS-I project is the surface salinity maximum in the 
North Atlantic within the North Atlantic Gyre. There is also a strong correlation between salinity maxima and evaporation (latent heat flux) minus precipitation magnitude (Schmitt, 2008). In addition, the salinity maximum is located slightly north of the evaporation minus precipitation maximum due in part to the northern Ekman transport of the saltier water mass (Schmitt et al., 1989). With the launch of the Aquarius satellite (Le Vine et al., 2007; Melnichenko et al., 2014) and SMOS mission (Kerr et al., 2010), salinity processes on a wide range of time-scales are of increasing interest to the oceanographic community. Understanding DWLs will continue to improve our understanding and estimation of moisture fluxes, and thus salinity in the upper ocean. Schanze et al. (2010) estimate that the evaporative loss of water from the ocean through air-sea fluxes is $13.0 \mathrm{~Sv}$. Compared to the 1.25 $\mathrm{Sv}$ discharge of fresh waters from rivers, atmosphere-ocean interactions account for nearly $90 \%$ of the oceanic freshwater cycle. The global water cycle has become an issue of great importance due to the changing climate. A recent analysis from observations using Argo floats and historic oceanographic data indicates an intensification of the water cycle due to global warming (Durack and Wijffels, 2010). Comparison with climate models show that in a climate just $2^{\circ} \mathrm{C}$ to $3^{\circ} \mathrm{C}$ warmer, an intensification of 16 to $24 \%$ of the global water cycle is possible (Durack et al., 2012). In a warming world, how the water cycle may change is vital to consider, and a better understanding of air-sea fluxes and upper ocean structure and variability is pertinent.

\subsection{Past Work and Present State of Knowledge}

The effect of the diurnal cycle on the ocean is not a new topic (e.g. Sverdrup et al., 1942; Saunders, 1967; Price et al., 1986), but new research continues to show the importance of resolving the diurnal cycle for processes on large range of time-scales; for example, the Madden-Julian Oscillation time-scale (Bernie et al., 2005; Woolnough et al., 2007; Bernie et al., 2008; Seo et al., 2014). Diurnal cycling in models has recently been argued to be important to air-sea coupling (Wang, 2001; Danabasoglu et al., 2006), and daytime stratification of the upper ocean directly affects the nighttime convection of the ocean (Brainerd and Gregg, 1993a,b).

Most research surrounding stable boundary layers is on the atmospheric-side of the geoscience community. Monin-Obukhov similarity theory (MOST; Monin and Obukhov, 1954) 
has been a useful tool for boundary layer scaling for 6 decades. MOST is a theory based on an empirical relationship between a depth scale based on surface parameters (here the MoninObukhov depth defined using Buckingham $\Pi$ theorem) and turbulent parameters within the boundary layer. Turbulent parameters are scaled as a function of a non-dimensional boundary layer depth, and that scaling is considered to be universal. For example, the surface fluxes, surface roughness, and distance from the surface can be used to estimate the TKE dissipation within the boundary layer. However, it has been noted that MOST does not properly describe the stably stratified boundary layer (Mahrt, 1998, 1999). Fernando and Hunt (1996) note that scaling laws must be carefully considered in the SBL, and that multiple length scales might exist, as opposed to a single length scale in unstratified turbulence. MOST does work well in conditions of weakly stable, neutral, or unstable boundary layers. Kumar and Sharan (2012) more recently have shown that MOST does not work in all cases of the SBL. Turbulence scaling and energetics under stably stratified regimes is still an area of active research, and more in-depth studies of the SBL are needed (atmospheric examples: Zilitinkevich et al., 2008; Savijärvi, 2009; Van de Wiel et al., 2008; Bergmann, 2011; Mahrt, 2011). Scaling of and turbulence within the DWL is considered in Chapter 2.

The SBL and diurnal warming may also be important to resolve on large spatial scales. For example, ocean fronts are modulated by diurnal warming (Obenour, 2013). Increased diurnal warming is associated with a decrease in persistent fronts and an increase in the number of fronts. It is hypothesized that oceanic fronts may be caused by spatially-varying diurnal warming as atmospheric conditions, which drive the warming magnitude, also vary spatially. The rectification of DWL associated with fronts and longer time-scales is considered in Chapter 4.

Diurnal warming has been shown to rectify in many different time-scales in the atmosphere and on the ocean surface (e.g., Shinoda, 2005; Clayson and Bogdanoff, 2013). However, there are relatively few examples of how diurnal warming may impact the lower frequency phenomena in the ocean. Fisher (2000) explored the impact of diurnally-varying vs. non-diurnally varying forcing over seasonal/monsoonal time-scales, and demonstrated the importance of considering diurnally-varying forcing for monsoonal time-scales to properly resolve boundary layer variability. Marshall et al. (1993) directly connect the mixed layer to the general circulation of the ocean. The net heat input into the mixed layer while the mixed layer is deepening can be entrained into the permanent thermocline. This subduc- 
tion period in the tropics is roughly 4 months, compared to 2 months in the mid-latitudes. Moreover, Clayson and Weitlich (2005) found that the mixed layer depth is overestimated on a daily scale when using a non-diurnally-varying solar flux. In some cases, the diurnal variations can be the same order of magnitude as the average depth of the mixed layer. The diurnal cycle can rectify into longer time-scales, and is important to consider even when examining low frequency variability.

One of the more comprehensive analyses of turbulence in the DWL is by Brainerd and Gregg (1993a,b). A very distinct daily pattern emerges from their observations. The ocean is well-mixed at the end of nighttime convection. Once the total surface heat flux become positive, the upper ocean begins to warm. A shallow diurnal thermocline or DWL exists and evolves throughout the day. Once the surface flux becomes negative and convection ensues, the DWL is no longer able to persist and is mixed into the deeper ocean. The DWL thickness is generally on the order of meters; however, under conditions of high diurnal warming, the diurnal thermocline may be present at depth of a meter or less (Soloviev and Lukas, 1997). These thin stable surface layers also trap momentum from the wind, generating shear across these layers (Kudryavtsev and Soloviev, 1990), as previously discussed. This has also been referred to as the "diurnal jet," but is physically just momentum trapped by the stable stratification of the strong vertical temperature gradient associated with diurnal warming (Price et al., 1986).

The structure of turbulence in the upper ocean is an area of active research, and the instruments used to observe turbulence in the ocean, such as microstructure profilers, have been used for decades. Brainerd and Gregg (1993a,b) used a microstructure profiler to study diurnal restratification of the upper ocean. Similarly, other groups have developed or are currently working with instrument platforms to provide high-vertical resolution microstructure data of the ocean, such as the Air-Sea Interaction Profiler (Ward et al., 2014; Callaghan et al., 2014; Sutherland et al., 2014). Similar to ASIP, the greatest benefit of the glider platform, used in this study, is the ability to take microstructure measurements through the surface during the ascent of the glider and the seclusion of the glider from potential anthropogenic turbulence generators, such as the ship.

Sutherland et al. (2016) made microstructure measurements, including TKE dissipation, during a French research cruise associated with the SPURS-I field campaign in late August and early September 2012. Using the ASIP, described above, and a custom buoy to measure 
near-surface currents, they observed an intensified near-surface currents and enhanced TKE dissipation associated with DWLs across six diurnal cycles. Creating a composite day of TKE dissipation, shear, and buoyancy frequency from the ASIP and current-measuring buoy, they scale their observations based on Monin-Obukhov Similarity Theory using surface observations from the ship. However, instead of assuming that shear follows the law of the wall scaling and thus allowing scaling based on only surface parameters and distance from the sea surface, they use observed shear and provide an modified version of MOST scaling of TKE dissipation. Based on this modified scaling and observations of the Richardson number generally being below unity, they conclude that shear instabilities associated with the DWL are the likely cause of the observed enhanced TKE dissipation. It is also important to note that all of the days during their deployments of ASIP were under relatively weak diurnal magnitudes of 0.2 to $0.5^{\circ} \mathrm{C}$, and on the two days of their campaign having wind speeds less than $2 \mathrm{~m} / \mathrm{s}$, conditions in which relatively large diurnal warming is expected, turbulence observations were not taken.

While at the surface the salient conclusion of the Sutherland et al. study and this dissertation appear to be different, many of the observationally-based statements allude otherwise. For example, Sutherland et al. use a modified MOST, which inherently relies on the appropriateness of a Monin-Obukhov depth, yet they conclude that estimates of Monin-Obukhov depth are less than their calculated mixed layer depth and does not "demonstrate any of the temporal variability show by the [mixed layer depth]." It is unclear that MOST scaling is appropriate under stably stratified conditions. Additionally, Sutherland et al. never directly prove that the mean shear is a plausible source of the enhanced TKE dissipation, but rather assume that the mean shear is the primary source through a turbulent shear production mechanism. In this dissertation, an alternative and plausible mechanism to explain some of the observed enhanced TKE dissipation, even under relatively weak diurnal warming, is provided. In addition, it is shown that surface-forcing alone, including shear associated with the DWL, cannot explain the observed enhanced TKE dissipation. Finally and most importantly, as pointed out in Chapters 2 and 3, the observations during the SPURS-I field campaign were taken in low wind speed conditions with relatively stronger diurnal warming, compared to the relatively weaker warming magnitudes of the Sutherland et al. study. The difference in observations and conclusions, as outlined in this dissertation, are potentially due to differences in observation conditions, including warming magnitude across the DWL. 
A more detailed comparison and analysis of the very recently released Sutherland et al. study is needed.

Direct Numerical Simulation (DNS) or Large Eddy Simulation (LES) are computationally expensive due to the much smaller size of the eddies in the SBL compared to the size of the eddies associated with nocturnal convection. As part of the Global Energy and Water Cycle Experiment (GEWEX) Atmospheric Boundary Layer Study (GABLS), Beare et al. (2006) performed an intercomparison of LES simulations of the SBL. Model results are sensitive to the grid resolution and sub-grid model choices, and the models tend to work best in conditions with moderately-stable boundary layers. In strongly stable boundary layers, the eddies are small compared to the grid spacing, and models do not work well. When the LES results are compared to a K-Profile Parameterization (KPP, Large et al., 1994) type firstorder parameterization, the results are promising; however, the KPP results are strongly dependent on an accurate calculation of boundary layer thickness. A detailed description of TKE dissipation estimates in this study using KPP are discussed in Chapter 2. McWilliams et al. (2009) found the KPP model to work appropriately in a "stably stratified regime." They did not specifically make note of DWLs, but rather looked at stable Ekman layers, which are a wind-driven boundary layer and were considered to be moderately stable.

Double-diffusion or salt fingering may contribute to mixing in the stably stratified nearsurface boundary layer (Soloviev and Lukas, 2006, p. 232). Hage and Tilgner (2010) found salt fingers in all stability regimes examined, demonstrating that salt fingering is possible in the stable boundary layer. Salt fingering is not considered in detail within this dissertation, but may be a feature of DWLs. However, TKE dissipation values observed with salt fingers are orders of magnitude below the observed values of the SPURS-I field campaign within DWLs.

Brainerd and Gregg (1993b) discuss that because Weller and Price (1988) did not find a minimum wind speed necessary for the development of Langmuir circulation, they may be responsible for some mixing in the SBL. By examining spatial scales less than a kilometer, Farrar et al. (2007) have also shown that internal waves can modulate the mixing of the DWL and impact diurnal warming. Hodges and Fratantoni (2014) found internal waves present in the DWL during the SPURS-I field campaign. The importance of internal waves in the DWL is the focus of Chapter 3. 


\subsection{Dissertation Objectives}

This dissertation discusses the structure and characteristics of turbulence in the near-surface ocean boundary layer utilizing novel observational techniques and theory. In particular, the focus is on the upper few meters within the DWL. This dissertation is motivated by the question "What is the observed structure of DWLs, and how does that compare to previous studies and current theory?" The objectives are:

1. to characterize the turbulence of the upper ocean under conditions of diurnal warming, specifically through (i) data analysis of the observations with respect to the diurnal cycle, (ii) evaluation of current ocean boundary layer theory such as Monin-Obukhov Similarity Theory, and (iii) one-dimensional ocean modeling with detailed consideration of surface forcing from the atmosphere.

2. to examine the existence of internal waves within the DWL and how internal waves within the DWL can create strong shear across the relatively thin layer, and potentially generate turbulence within that layer.

3. to explain how horizontal transport associated with shear across the DWL coupled with the diurnal cycle of convection in the upper ocean is a mechanism for effective lateral diffusion within the mixed layer.

Examining the observations and using simple, idealized, theoretical models will provide physical insight into the importance of resolving diurnal warming. It is the primary goal of this dissertation to characterize the structure and variability of the DWL with a specific focus on turbulence and internal waves, while in addition examining how DWLs can rectify into longer time-scales and larger spatial scales. Additionally, there is a focus on understanding the importance of diurnal variability and associated DWLs to broader ocean implications, potentially with global significance.

\subsection{Dissertation Outline}

Each chapter comprises a stand-alone discussion suitable for publication as a research article, and thus each chapter has a detailed background, data, and methodology. In order to reach the dissertation objectives, Chapter 2 focuses on the observation of turbulence from 
the gliders, specifically turbulent kinetic energy dissipation, and modeling of the DWL. The enhanced dissipation is confined to the upper 30 meters during the daytime, and the diurnal cycle of dissipation is oppositely-phased from previous studies. The largest values are observed during the daytime. Two different one-dimensional upper ocean models (Kantha-Clayson and Price-Weller-Pinkel) do not reproduce the enhanced daytime dissipation, nor does Monin-Obukhov Similarity Theory. MOST does properly estimate TKE dissipation under destabilizing surface forcing (net surface heat flux is cooling the ocean). Both one-dimensional models reproduce a DWL shear caused by momentum trapped by diurnal stratification, but at shallower depths than observed. During the daytime, the upper ocean is, on average, dynamically stable (Richardson number $>0.25$ ) to Kelvin-Helmholtz instability. The most surprising result of this study is that the largest observed TKE dissipation is associated with the most gravitationally stable conditions. Additionally, neither the energy of the observed mean current nor wind work appear to the be the sole source of energy for the TKE dissipation. It is unlikely that surface-forced shear alone, including diurnal shear, is responsible for the observed enhanced daytime TKE dissipation.

Chapter 3 considers the importance of internal waves within the DWL, when conditions become favorable for propagating internal waves within the layer. Internal waves propagating from the deep ocean are shown to enhance the vertical shear across the DWL. Deep internal waves with significant energy and decay scales larger than the mixed layer induce horizontal motion in the near-surface ocean. Beginning with the concepts introduced in D'Asaro (1978) detailing the effects of deep internal waves on horizontal motion in a deep mixed layer, this work expands upon this model by including a stratified near-surface layer as observed in the daytime upper ocean. Internal wave-induced velocities from waves with specific frequencies and wavenumbers can cause local shear instability. It is shown that internal waveinduced velocities are a plausible source of turbulence and energy associated enhanced TKE dissipation. Increased predicted shear from internal waves within the DWL are coincident with increased observed near-surface stratification and enhanced TKE dissipation.

Chapter 4 considers the rectification of diurnal warming into larger spatial and temporal scales. It is shown that advection in the near-surface during the daytime converts horizontal gradients into vertical gradients. Coupled with vertical mixing at night within the mixed layer, it is a mechanism for effective lateral mixing over a day on the scale of 1-10 km. This is illustrated with a simplified representation of the mechanism. Additionally, an effective 
submesoscale horizontal diffusivity for the mixed layer is estimated. The calculated effective diffusivity associated with this mechanism is $1-100 \mathrm{~m}^{2} / \mathrm{s}$, depending on the advective timescale, and depth and speed of the DWL current. This daily cycle may be an important mechanism for smoothing lateral inhomogeneities within the mixed layer. Additionally, how the advection associated with the DWL may locally enhance or suppress the diurnal warming observed at a location is considered.

Lastly, Chapter 5 summarizes the conclusions of the dissertation as a whole, and considers the broader implications of the work. Future research directions are also discussed. 


\section{Chapter 2}

\section{Potential mechanisms for observed}

\section{enhanced turbulent kinetic energy \\ dissipation in the daytime upper \\ ocean during SPURS-I}

\section{$2.1 \quad$ Introduction}

The near-surface ocean is complicated by a variety of phenomena with overlapping time periods and spatial scales, including surface gravity waves, internal waves, inertial oscillations, and diurnal cycling (Moum and Smyth, 2001). During the daytime, incoming solar radiation acts to stabilize the photic zone of the ocean. The upper few meters of the ocean warm and then cool over the course of a diurnal cycle. Under very light wind speed conditions (less than $2 \mathrm{~m} / \mathrm{s}$ ) the upper few meters of the ocean can warm several degrees (Stramma et al., 1986; Yokoyama and Konda, 1996). For these conditions, turbulence is thought to be suppressed by the positive buoyancy flux due to penetrative solar radiation (Webster et al., 1996; Soloviev and Lukas, 1997). Convective mixing that occurs at the surface due to net heat loss by turbulent and radiative fluxes is suppressed by the volume absorption of solar radiation over that thin layer (Soloviev and Lukas, 1997), resulting in a strongly stable near-surface layer.

Sea surface temperature (SST) is considered the most important variable controlling 
air-sea energy exchange (Large et al., 1994). Warming that occurs at the sea surface on a daily basis will generate a coupled response. The atmospheric and oceanic boundary layers respond to changes in momentum, moisture, and heat which are transferred through wind stress, and turbulent and radiative fluxes, at the air-sea interface (e.g. Price et al., 1986). Therefore, the structure of the near-surface layers of the atmosphere and ocean affect the magnitude of the transfer, and in turn the magnitude of the transfer changes the structure of the near-surface layers. Under windy and destabilizing surface forcing conditions, the structure and processes of the boundary layers have been observed and well studied (see Wyngaard et al., 1971; Shay and Gregg, 1986; McPhee, 1994; McNaughton, 2006).

However, substantial questions remain regarding the stably stratified upper ocean boundary layer, such as the appropriate magnitude and scaling of turbulent quantities, partly due to a lack of necessary observations. New instrument platforms and techniques, such as the Air-Sea Interaction Profiler (ASIP, Ward et al., 2014) and Rockland Scientific MicroRider (used in this study, e.g. Fer et al., 2014; Clayson et al., 2016b), provide novel ways to observe and investigate the upper 10 meters of the ocean and relatively shallow stable layers. Specifically, autonomous platforms have provided observations of turbulence near the surface of the ocean that could not be observed by traditional shipboard microstructure profiling (Callaghan et al., 2014).

Most theory and understanding of the stable boundary layers comes from meteorological experiments, as the stable layers in the atmosphere are orders of magnitude thicker than those found in the ocean (e.g. CASES-99; Poulos et al., 2002). Boundary layer theory, such as Monin-Obukhov Similarity Theory (MOST) is based on observations of the atmosphere (Monin and Obukhov, 1954; Foken, 2006) and has been applied to the ocean (Large et al., 1994; Wenegrat and McPhaden, 2015). MOST is a theory that considers the effect of a buoyancy flux at the surface on the boundary layer of the atmosphere or ocean. The applicability of MOST to the ocean, especially under stabilizing conditions (also to the atmosphere under these conditions), is still a topic of debate (e.g. Cheng et al., 2005; Tedford et al., 2014), but the theory provides a starting point to describe the turbulence of the upper ocean. The strongest turbulence occurring within the diurnal cycle (outside of strong wind mixing events) is generally expected within the active convective mixed layer. This occurs during the nighttime when the net heat flux cools the near-surface ocean creating a positive density anomaly at the surface and Rayleigh-Taylor (convective) instability (Shay 
and Gregg, 1986). These conditions are relatively well-understood and accurately predicted by boundary layer theory such as MOST. The focus of this study is on conditions with stabilizing surface forcing during the daytime.

Clayson et al. (2016b) show occasional enhanced Turbulent Kinetic Energy (TKE) dissipation and dissipation of thermal variance associated with diurnal warm layers (DWLs) in the subtropical North Atlantic Ocean during two cruises of the Salinity Processes in the Upper ocean Regional Study (SPURS-I) field campaign. The same dataset is used in this study. Their results contrast with some previous studies, which found that under stable conditions TKE dissipation (or some other indicator of turbulent mixing) is suppressed (Moum et al., 1989; Brainerd and Gregg, 1993a; Peters et al., 1994; Soloviev et al., 2002; Soloviev and Lukas, 2006).

Additionally, DWLs are warmer and saltier layers atop a relatively cooler and fresher ocean. Therefore, double-diffusion or salt fingering may contribute to mixing in the stably stratified near-surface boundary layer (Soloviev and Lukas, 2014, p. 232). Salt fingers may also contribute to the observed enhanced TKE dissipation however, the levels of TKE dissipation observed under clear salt fingering regimes $\left(\epsilon \sim 10^{-7} \mathrm{~W} / \mathrm{kg}\right)$ are orders of magnitude smaller than observed during the SPURS-I field campaign within DWLs under conditions of weak winds and high solar insolation (St. Laurent and Schmitt, 1999).

Brainerd and Gregg (1993a) observed continuous enhanced dissipation $\left(\epsilon>10^{-6} \mathrm{~W} / \mathrm{kg}\right)$ in the near-surface ocean compared to the values observed in the deeper ocean. They also observed a reduction in turbulence with increased stratification in the diurnal thermocline (5-10m), but call their data 'suspect' due to the possible influence of the ship's wake (one of the main issues with traditional microstructure profiling, see Hodges and Fratantoni 2014, Fig. 1). In addition, the enhancement was observed during the daytime and nighttime. The enhanced dissipation is attributed to three possibilities: contamination by the wake of the ship, shear generated by wind forcing, or surface gravity waves. Through scaling and not direct observations of shear, they conclude that shear production alone cannot explain the magnitude of dissipation in the near-surface. In contrast, in this study the glider platform allows for the exclusion of the ship's wake as a source of turbulence, and an upward-looking ADCP provides hourly estimates of shear in the near-surface.

During stabilizing surface forcing conditions, momentum transferred from the wind is trapped in the near-surface ocean creating a diurnal warm layer shear (Price et al., 1986). 
The input of momentum into a relatively thin surface layer eventually generates conditions in which the Richardson number drops below critical and the layer overturns. This process of momentum input and eventual overturning is the self-regulating state of the diurnal thermocline (Kudryavtsev and Soloviev, 1990; Soloviev and Lukas, 2006), and turbulence generation mechanism in the stable boundary layer. However, turbulence estimates associated with the diurnal warm layer shear, such as Wang (2001), are much smaller than observed during the SPURS-I field campaign. The results of this study are not inconsistent with a recent study in the same region as SPURS-I just a few weeks before the first cruise (Sutherland et al., 2016). Using the ASIP, Sutherland et al. (2016) show enhanced TKE dissipation in the upper ocean and relate it to shear across the DWL, using boundary layer scaling. However, their observations were under different wind speed conditions and here it will be demonstrated that the shear of the diurnal warm layer alone cannot explain all of the observed enhanced TKE dissipation. A more detailed analysis of the Sutherland et al. study is provided in Chapter 1, Section 2.

The focus of this paper is on surface forcing and the diurnal warm layer shear, using models and theory of the near-surface ocean. We show that the local surface forcing alone appears inadequate to explain the enhanced dissipation rates observed during the SPURSI field campaign. A follow-on to this study will examine how internal waves may induce strong near-surface velocities during diurnal warm layer conditions in which enhanced TKE dissipation is observed (Chapter 3).

Section 2 contains details on the observational platforms and cruises. The diurnal cycle of TKE dissipation in the near-surface ocean is analyzed in Section 3. A discussion of canonical surface-forced phenomena using two different one-dimensional models and MoninObukhov Similarity Theory, and their failure to explain the observed pattern of turbulence, is provided in Section 4. Section 5 details the diurnal warm layer shear and self-regulating state of the diurnal thermocline, and provides evidence to rule out the likelihood of a local surface-forced phenomenon alone as a cause of the enhanced TKE dissipation. A summary of results and brief discussion are provided in Section 6 . 


\section{SPURS-I Field Campaign \& Glider Profile Density}

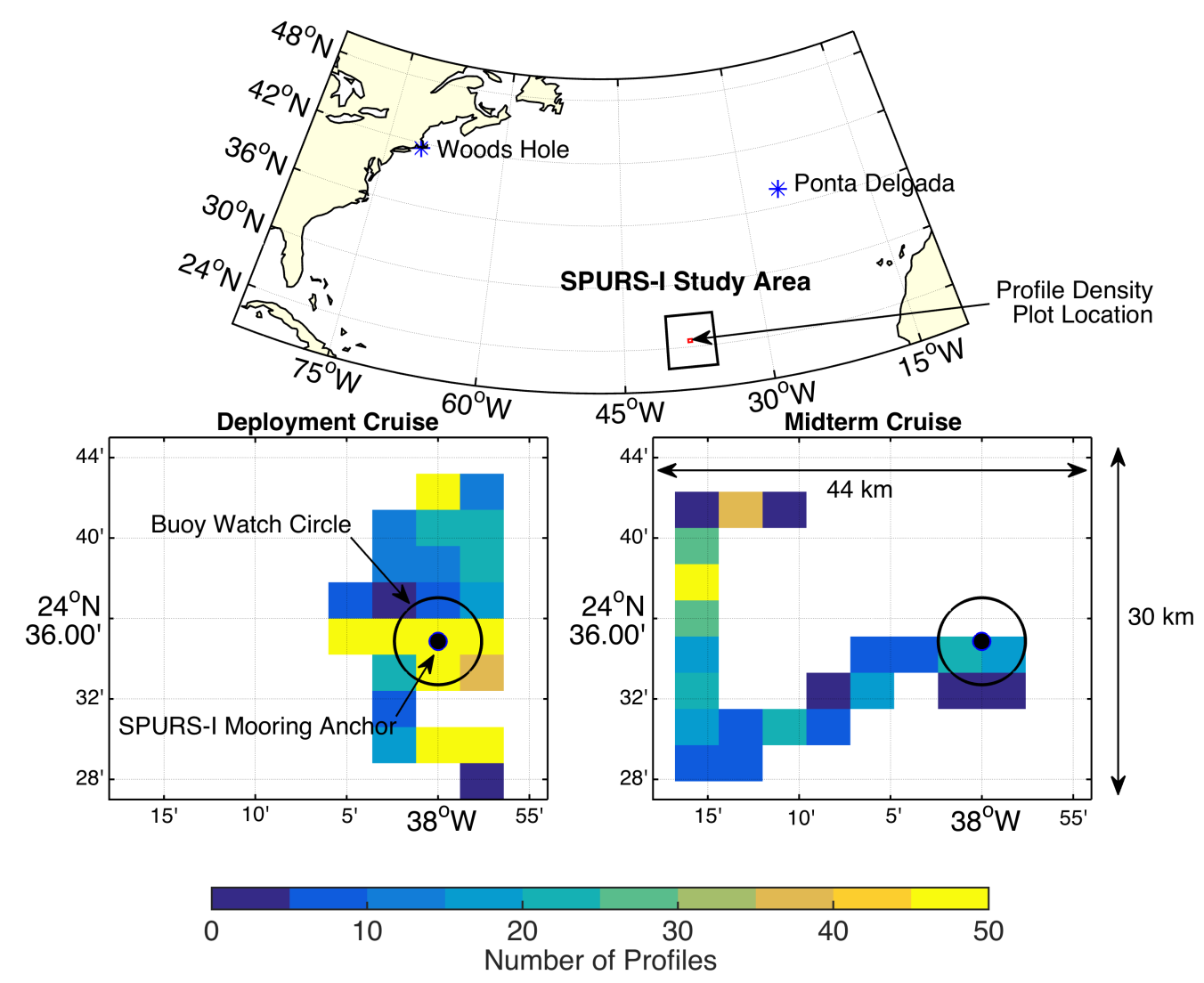

Figure 2-1: Location of the SPURS-I field campaign site (top), and density of glider profiles in the area shaded by color in 2.5' x 2.5' bins near the SPURS-I mooring (black dot with black watch circle) for the deployment (9/15/2012 - 10/5/2012) and midterm (3/25/2013 4/6/2013) cruises.

\subsection{Observations and cruise details}

The data were collected as part of a year-long field campaign in the surface salinity maximum of the North Atlantic Ocean (Fig. 2-1), as part of the Salinity Processes in the Upper ocean Regional Study (herein referred to as SPURS-I). Three research cruises were associated with the SPURS-I field campaign: a deployment cruise in fall 2012 (denoted as such since most assets were deployed during this cruise), mid-term cruise in spring 2013, and recovery cruise in fall 2013. This analysis focuses on a year-long SPURS-I mooring record moored at $24.5^{\circ} \mathrm{N}$, $38^{\circ} \mathrm{W}$, and glider data collected during two cruises. 


\subsubsection{SPURS-I Mooring}

The central SPURS-I mooring (Farrar et al., 2015) provides high temporal- and verticalresolution observations of the upper ocean (every 5 minutes, 31 CTDs in the upper 200 meters). Three upward-facing ADCPs (at 18, 75, and 145 meters depth) and a point current meter at 3.5 meters provide current measurements of the upper 150 meters. The CTD data are gridded by interpolation to a consistent 5-minute grid, and the ACDP data are hourly (5-minute average current every hour). The records from the two upper-ocean ADCPs and the point current meter at 3.5 meters were combined to create a continuous grid of upper ocean currents. The closest CTD and current measurement to the surface are $0.75 \mathrm{~m}$ and $3.5 \mathrm{~m}$, respectively. This set of temperature, salinity, and current data has been used in an upper ocean heat and salt budget study (Farrar et al., 2015).

The SPURS-I central surface mooring provides one-minute atmospheric and surface ocean data. The surface component includes an IMET system to measure a suite of meteorological variables including temperature, humidity, wind speed and direction, shortwave and longwave radiation, precipitation, wave information, and sea surface temperature and salinity (Hosom et al., 1995; Colbo and Weller, 2009). A net surface flux is calculated and used throughout this study as

$$
Q_{N}=Q_{L}+Q_{B}+Q_{P}+Q_{L W}+Q_{S W}
$$

where a positive flux is into the ocean, and $Q_{N}$ is the net surface flux, $Q_{L}$ is the latent heat flux, $Q_{B}$ is the sensible heat flux, $Q_{P}$ is the heat flux due to temperature difference between the ocean surface and precipitation, $Q_{L W}$ is the outgoing longwave radiation, and $Q_{S W}$ is the incoming shortwave where the turbulent fluxes are estimated from the COARE 3.0 algorithm (Fairall et al., 2003).

\subsubsection{Slocum Gliders and Microstructure}

During the deployment and mid-term cruises, two Slocum gliders with Rockland Scientific MicroRiders to measure microstructure were deployed simultaneously, with one of the two gliders profiling frequently near the surface. A total of 3,692 profiles, ranging from 60 - 200 meters in depth were obtained over 31 days. Microstructure measurements from autonomous underwater gliders are a relatively recent methodology; Slocum gliders are more commonly 
used as an autonomous platform for temperature and salinity measurements (Schofield et al., 2007). Peterson and Fer (2014) and Fer et al. (2014) found the microstructure data from gliders to be high quality and the gliders a useful platform.

In addition to the gliders with microstructure probes, a Rockland Scientific Vertical Microstructure Profiler (VMP, used in many studies such as St. Laurent and Thurnherr, 2007; Waterman et al., 2013) was deployed during both cruises. During the first cruise a deep VMP provided microstructure to $1500 \mathrm{~m}$, and during the second cruise a tethered VMP-500 provided a greater number of profiles of the upper $300 \mathrm{~m}$ of the ocean. Since VMPs do not provide accurate estimates of microstructure until they reach free fall and must be deployed from a ship, the data from the upper 10 meters are typically not analyzed when using this observational platform (Callaghan et al., 2014).

This analysis utilizes both glider and VMP profiles, and the focus is on two glider missions closest to the central mooring in order to use the meteorological information provided by the atmospheric instruments on the buoy (Table 2.1). This narrows the focus to 19 days with microstructure profiles every 8-15 minutes. The mooring had a 4.3 nautical mile $(8.0 \mathrm{~km})$ watch circle (horizontal range of the buoy at the surface) and most of the profiles during the first cruise were within that circle. The location density of profiles in which the gliders were near the central mooring are shown in Fig. 2-1. The glider profiles were generally within $40 \mathrm{~km}$ of the mooring. The gliders were ballasted light to allow the MicroRider to breach the surface, and profiled on both upcasts and downcasts. Due to a CTD data storage issue aboard the glider during the first cruise, the temperature and salinity data from the mooring are substituted for missing data in a 16 hour period from 0200-1600 Z on September 26, 2012.

\subsection{The diurnal cycle of TKE dissipation}

The microstructure observations during the SPURS-I cruises occasionally reveal high values of dissipation of turbulent kinetic energy $\left(\epsilon\right.$, greater than $1 \times 10^{-5} \mathrm{~W} / \mathrm{kg}$ ) and dissipation of temperature variance ( $\chi$, greater than $1 \times 10^{-6}{ }^{\circ} \mathrm{C}^{2} / \mathrm{s}$ ) in the upper 10 meters of the ocean during the daytime. Clayson et al. (2016b) detail the microstructure measurements and signal observed during the SPURS-I field campaign. Near-surface observations of TKE dissipation and dissipation of thermal variance from the gliders near the central mooring 
Table 2.1: Glider missions during the deployment and midterm cruises, including the instrument type, dates of mission, number of profiles, and mission location description. While all the data is used in the analysis of the diurnal cycle of TKE dissipation, only those deployments near the central mooring are used throughout the rest of the study.

\begin{tabular}{ccccc} 
Cruise & Instrument & Dates & $\#$ Profiles & Location \\
\hline \hline Deployment (2012) & Shallow Glider & $9 / 22-10 / 4$ & 1469 & Central Mooring \\
Deployment (2012) & Deep Glider & $9 / 15-10 / 4$ & 751 & Mooring Triangle \\
Deployment (2012) & Deep VMP & $9 / 18-9 / 27$ & 24 & Study Area \\
\hline Midterm (2013) & Shallow Glider & $3 / 25-3 / 30$ & 777 & Central Mooring \\
Midterm (2013) & Shallow Glider & $3 / 31-4 / 5$ & 211 & Study Area \\
Midterm (2013) & Deep Glider & $3 / 25-4 / 5$ & 484 & Mooring Triangle \\
Midterm (2013) & VMP-500 & $3 / 28-4 / 6$ & 145 & Study Area \\
\hline \hline
\end{tabular}

during the first two cruises of the field campaign are provided in Fig. 2-2. The high dissipation during the daytime is present throughout the record when diurnal warming is observed, with larger values than associated with nocturnal convection.

The daytime signal of dissipation from the gliders is observed to 30 meters during the deployment cruise and to just over 20 meters during the midterm cruise. The signal is also observed in dissipation of thermal variance. The VMP data from the first cruise, which are the deepest microstructure observations made in the region during the SPURS-I field campaign, show enhanced upper ocean turbulence (Fig. 2-3) and relatively weak turbulence deeper in the ocean. There were not enough VMP profiles from the first cruise to separate day vs. night and windy versus calmer conditions. VMP data from the second cruise also reveal enhanced TKE dissipation in the upper ocean and a few of the profiles revealed enhanced dissipation during the daytime compared to the nighttime. However, since the VMP was tethered and deployed off the stern of the ship, the near-surface measurement may be contaminated by the ship's wake. The observations from the mid-term cruise are only to $300 \mathrm{~m}$.

When the wind speed is greater than $5 \mathrm{~m} / \mathrm{s}$, relatively weak diurnal warming is expected because of mechanical mixing by the wind (Webster et al., 1996). Fig. 2-4 shows the cycle of dissipation for the upper 30 meters from the SPURS-I microstructure separated into conditions below and above $5 \mathrm{~m} / \mathrm{s}$. Under conditions with wind speeds less than $5 \mathrm{~m} / \mathrm{s}$ there is a small enhancement of TKE dissipation during the daytime, but a clear cycle is not present (Fig. 2-4b). This signal of enhanced dissipation in the daytime upper ocean is 

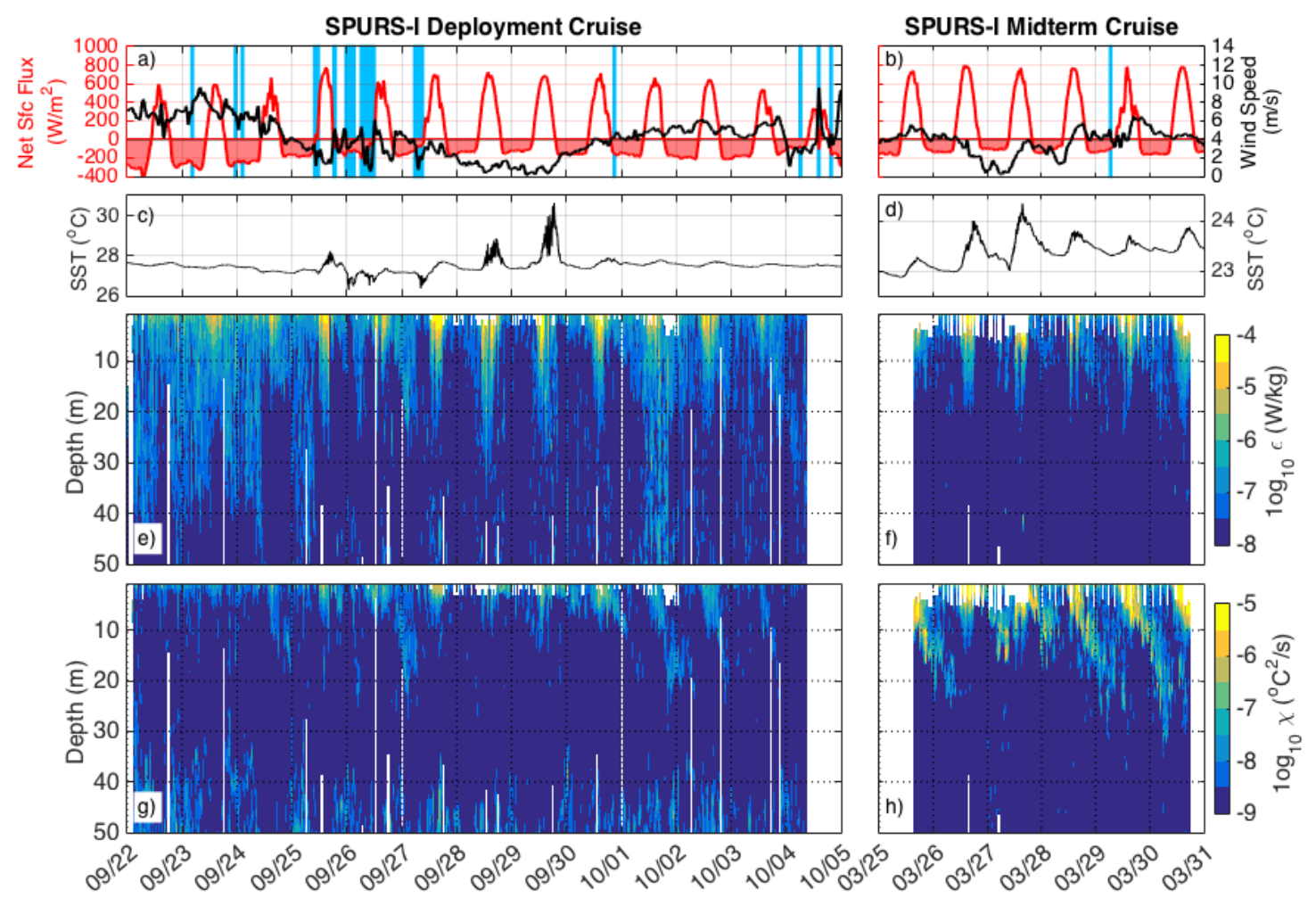

Figure 2-2: The meteorological conditions at the SPURS mooring $(a, b)$ with the net surface heat flux in red, wind speed in black, and times of active precipitation in light blue. Sea surface temperature $(c, d)$, turbulent kinetic energy dissipation $(e, f)$, and dissipation of thermal variance $(\mathrm{g}, \mathrm{h})$ from the gliders during the deployment (left-side, 9/15/2012 - 10/5/2012) and midterm (right-side, 3/25/2013 - 4/6/2013) cruises. 

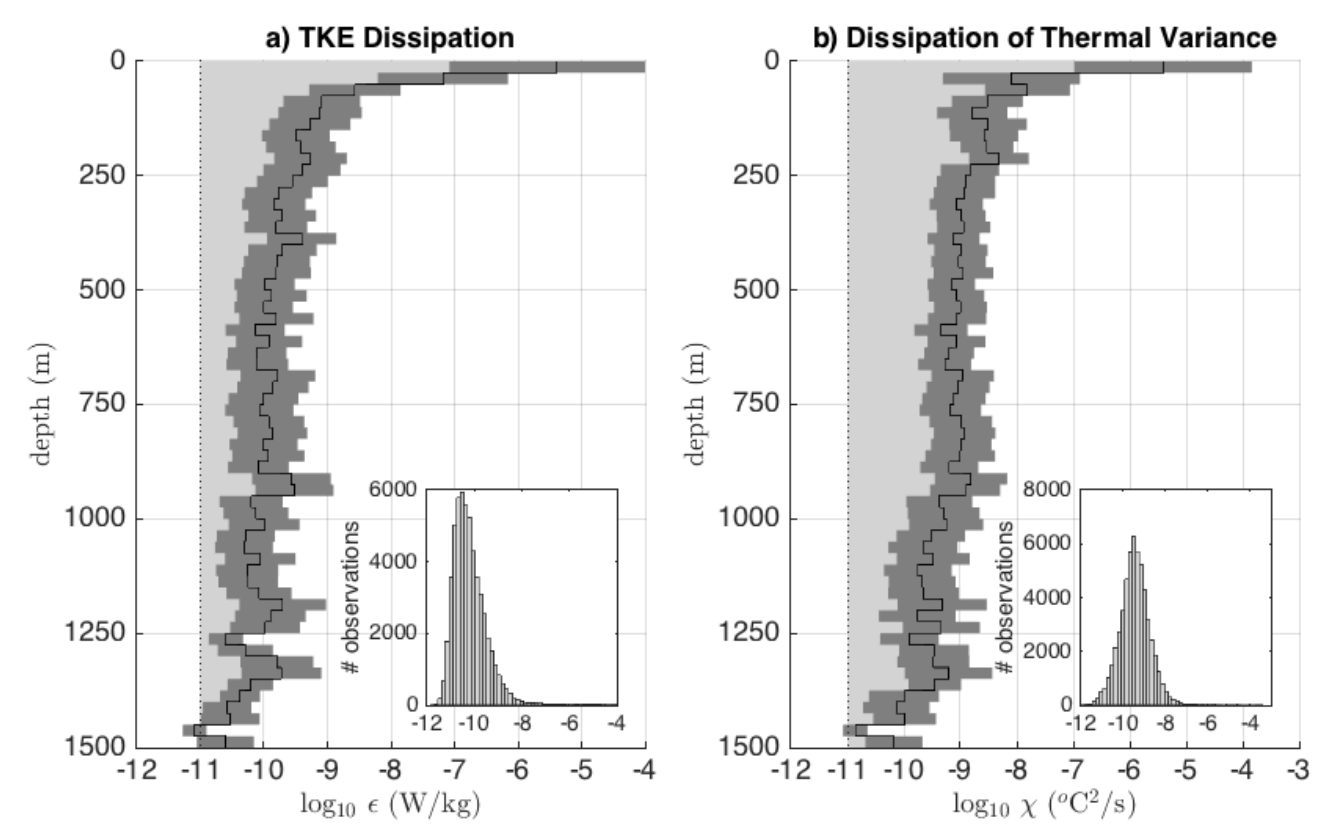

Figure 2-3: Average profile of TKE dissipation and dissipation of thermal variance (black line) with standard deviation (dark grey) during the deployment cruise from the deep VMP. Insets of a histogram of all observations for all depths are plotted as well.

not in agreement with previous observations (e.g. Soloviev and Vershinsky, 1982; Soloviev et al., 1988; Moum et al., 1989; Brainerd and Gregg, 1993a,b). However, these studies used traditional microstructure platforms that cannot accurately measure the near-surface ocean (roughly the upper 10 meters).

For the SPURS-I data from 30-80 meters under windy conditions, a cycle with suppressed daytime turbulence is observed in agreement with Brainerd and Gregg (1993a). Moum et al. (1989) also observed a cycle with suppressed daytime TKE dissipation in 10-100 meter averages. However, their field campaign occurred in a region of the ocean with deep shear caused by the Equatorial Undercurrent (EUC) that provides significant source of mean kinetic energy (that can be converted to TKE) not present in other parts of the world oceans, including the North Atlantic Ocean. Smyth et al. (2013) theorize that the descent of surface layer shear interacts with the mean shear of the Equatorial Undercurrent causing the enhanced dissipation, which is in agreement with previous studies of TKE dissipation in the EUC (Clayson and Kantha, 1999).

Brainerd and Gregg (1993a) found a cycle of suppressed daytime turbulence in the nearsurface ocean $(\sim 7-10 \mathrm{~m})$ under conditions with relatively weak winds (less than $5 \mathrm{~m} / \mathrm{s})$. In 

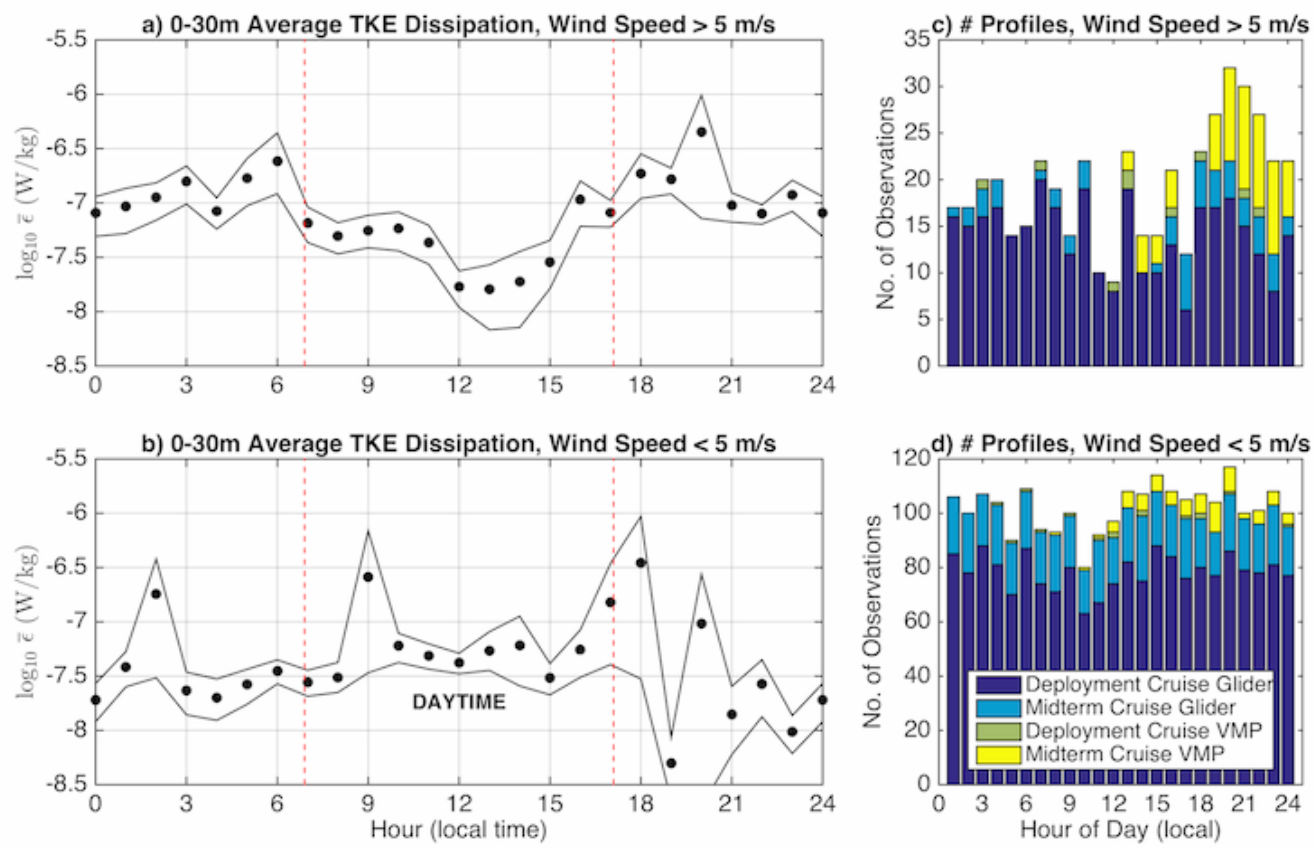

Figure 2-4: Hourly averages of the average TKE dissipation of the upper 30 meters of the ocean during (a) wind speed greater than $5 \mathrm{~m} / \mathrm{s}$, and (b) less than $5 \mathrm{~m} / \mathrm{s}$. The black circles indicate the mean, and the black lines indicate the $95 \%$ confidence limits, estimated by bootstrapping. The vertical red dashed lines indicate when the average net surface heat flux changes sign. The number of profiles used in each hourly average in (a) and (b) are shown in $(\mathrm{c})$ and $(\mathrm{d})$, respectively.

contrast, during SPURS-I under similar wind speed conditions, enhanced daytime dissipation was shown (again, SPURS-I observations are also able to resolve conditions much closer to the surface). Under conditions with wind speeds less than $5 \mathrm{~m} / \mathrm{s}$ in the SPURS-I data, using an average of the upper 30 meters a slight enhancement of dissipation during the daytime is visible (Fig. 2-4b). Composite average dissipation by depth and buoyancy frequency are shown in Fig. 2-5 for wind speeds less than $5 \mathrm{~m} / \mathrm{s}$ for day and night, and wind speeds greater than $5 \mathrm{~m} / \mathrm{s}$ (note that there are not enough observations to split higher wind conditions into day and night). The largest values of TKE dissipation are observed during the daytime (Fig. 2-5a) when wind speed are less than $5 \mathrm{~m} / \mathrm{s}$. The largest values during the daytime are orders of magnitude greater than the values observed during nocturnal convection at low wind speeds (Fig. 2-5b) and when wind speeds are greater than $5 \mathrm{~m} / \mathrm{s}$ (Fig. 2-5c).

The high stratification associated with nighttime light wind conditions are observed early in the night before nocturnal convection mixes the daytime stratification (Fig. 2-5a, see also 

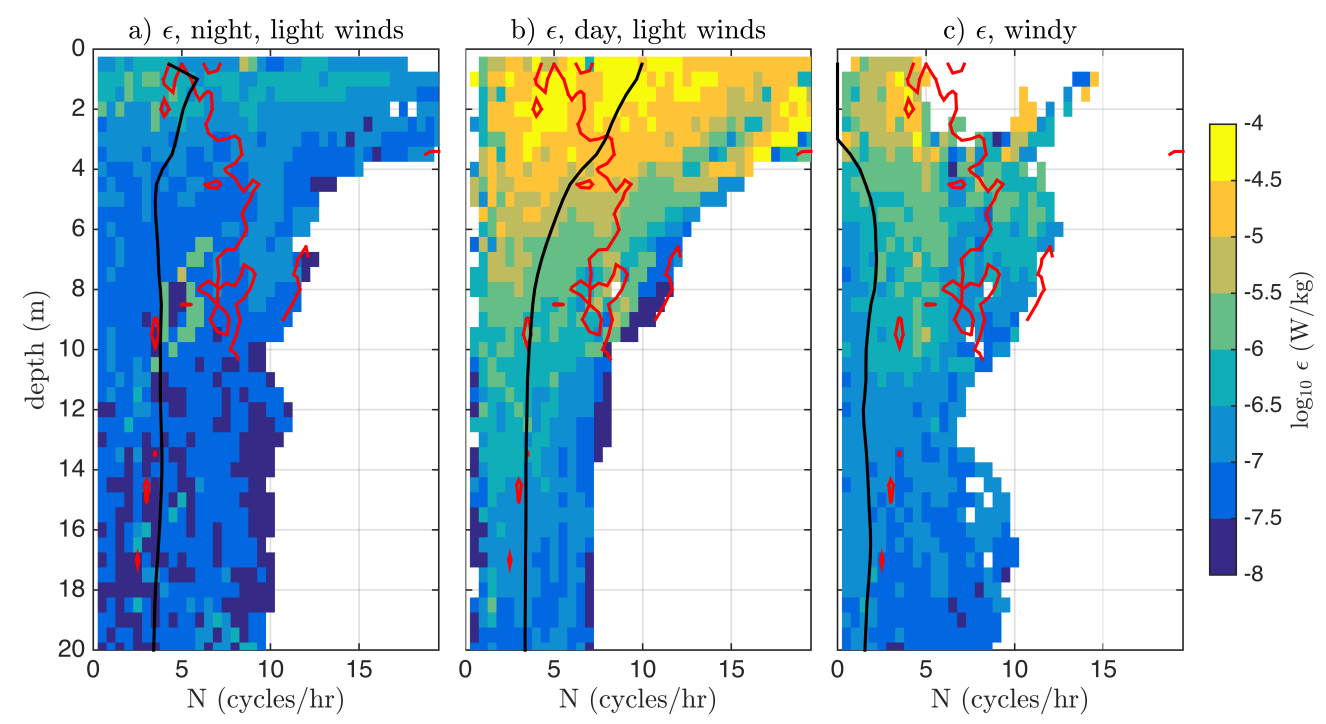

Figure 2-5: Composite average TKE dissipation shown by color shading binned by depth and buoyancy frequency (cph) for (a) nighttime light wind conditions ( $Q_{n e t}<0$, wspd $<5 \mathrm{~m} / \mathrm{s}$ ), (b) daytime light wind conditions ( $\mathrm{Q}_{\text {net }}>0$, wspd $<5 \mathrm{~m} / \mathrm{s}$ ), and (c) windy conditions (wspd $>5 \mathrm{~m} / \mathrm{s}$ ). The black lines indicate the average buoyancy frequency profile for each condition. The red contour shows where the ocean warms, on average, more than $0.25^{\circ} \mathrm{C}$ from the value at sunrise at each depth, based on the light wind daytime composite, and is plotted on all three for reference.

Fig. 2-12). During these time periods, low wind speeds tend to reduce the air-sea fluxes causing shallower and weaker convection allowing the diurnal thermocline to persist into the night. The descent of nocturnal convection is most easily observed in $\chi$ (Fig. 2-2) during the last few days of the deployment cruise and all of midterm cruise, but not during the very low wind speed period during the deployment cruise associated with the highest observed values of near-surface stratification (September 28 - 30). In contrast, under windy conditions high stratification is not observed near the surface due to mechanical mixing by momentum input from the winds (Fig. 2-5c).

\subsection{Canonical 1D view of surface forced mechanisms}

Momentum and heat fluxes are the primary drivers of the variability in the near-surface ocean (e.g. Large et al., 1994; Callaghan et al., 2014). The momentum input from the wind is an important source of shear production and thus TKE in the upper ocean. The equation 
for TKE is

$$
\begin{gathered}
\frac{\partial k}{\partial t}+\bar{u}_{j} \frac{\partial k}{\partial x_{j}}=-\frac{1}{\rho_{0}} \frac{\partial \overline{u_{i}^{\prime} p^{\prime}}}{\partial x_{i}}-\frac{\partial \overline{u_{j}^{\prime} k}}{\partial x_{j}}+\nu \frac{\partial^{2} k}{\partial x_{j}^{2}}-\overline{u_{i}^{\prime} u_{j}^{\prime}} \frac{\partial \bar{U}_{i}}{\partial x_{j}}-\nu \frac{\partial \overline{u_{i}^{\prime}} \frac{\partial u_{i}^{\prime}}{\partial x_{j}}}{\partial x_{j}}+\delta_{i 3} \overline{u_{i}^{\prime} b^{\prime}} \\
\mathrm{P}
\end{gathered}
$$

where $k$ is turbulent kinetic energy, $u$ in the velocity tensor, $p$ is pressure, $\nu$ is the kinematic viscosity of seawater, and $b$ is the buoyancy $(b=-g \rho)$. Over-bar terms are the ensemble mean quantities and primed terms are the perturbations from the mean (where the mean of the perturbation is defined as zero).

Outside of the convective (thermal) sublayer during the daytime when the upper ocean is stably stratified, the buoyancy production/destruction term (B) must be negative because turbulence acts to destroy the mean vertical density gradient. The non-labeled terms of Eq. 2.2 are transport or storage, and do not globally create nor destroy TKE. Dissipation $(\epsilon)$ is always a sink of TKE (negative sign before the positive-definite dissipation term). Assuming a production-dissipation balance, or that the transport and storage terms are negligible, any mechanism responsible for the enhanced TKE dissipation must locally generate turbulence through shear production $(\mathrm{P})$. In this section, the observed enhanced dissipation is analyzed with respect to surface-forced shear mechanisms through a comparison with the results of two one-dimensional upper ocean models and Monin-Obukhov Similarity Theory.

\subsubsection{Modeling the near-surface ocean}

Two one-dimensional ocean models are used to predict the characteristics of the nearsurface ocean from surface forcing: the Kantha-Clayson 2nd-moment turbulence closure model (Kantha and Clayson, 1994, 2004, hereafter KC) and Price-Weller-Pinkel model (Price et al., 1986, hereafter PWP). The models have different approaches to predict the mixed layer characteristics from provided surface forcing and an initial profile. $\mathrm{KC}$ is an improved Mellor-Yamada (Mellor and Yamada, 1974) second-moment closure model that directly predicts some turbulence variables, such as TKE dissipation. KC has been used extensively to study diurnal warming (e.g. Clayson and Weitlich, 2005; Embury et al., 2012; Clayson and Bogdanoff, 2013; Scanlon et al., 2013). PWP is a dynamical instability model that utilizes a Richardson number mixing scheme. PWP has been shown to reproduce diurnal warm layers well (Price et al., 1986; Weller and Anderson, 1996; Prytherch et al., 2013). PWP however, 
does not explicitly solve for turbulent kinetic energy dissipation. Additional calculations are done following Schudlich and Price (1992) to estimate TKE dissipation from the model (Appendix A).

Both models are run with a 15 minute time step and $0.1 \mathrm{~m}$ vertical resolution using 15 minute smoothed data from the surface mooring (estimated wind stress and turbulent fluxes, observed shortwave radiation) and a linearly interpolated initial profile from the mooring at the time closest to the start time of the model run. The model is run such that a day of spin-up is provided before the results are analyzed.

A 2-band solar extinction parameterization is used with Jerlov type IA coefficients (5 m and $40 \mathrm{~m}$ e-folding extinction scales for the bands), appropriate for subtropical open ocean regions such as the Sargasso Sea (Jerlov, 1976). A 9-band extinction parameterization was also used for the model runs (Paulson and Simpson, 1981) and it does change the structure of the near-surface temperature. However, it did not change the turbulence characteristics, such as TKE dissipation, by more than 1\%. The details of the differences due to solar extinction parameterizations are not the focus of this study, but are important for accurately reproducing SST under conditions of near-calm winds and high solar insolation. In conditions with little mixing, the vertical profile of temperature should match the profile of solar extinction relatively well and thus is important to properly consider. The influence of the selection of a solar extinction parameterization has been of importance to the oceanographic community for over 30 years (Dickey and Simpson, 1983; Gentemann et al., 2009), and still requires further investigation.

The model results are compared with the closest-to-surface observations ( $0.75 \mathrm{~m}$ model results compared to $0.75 \mathrm{~m}$ mooring observations) of temperature, salinity, and current (Fig. 2-6). PWP appears to underestimate mixing, causing stronger than observed diurnal warming (Fig. 2-6a,b). For the large diurnal warming days (greater than $1^{\circ} \mathrm{C}, 9 / 28,9 / 29$, $3 / 26$, and $3 / 27)$, PWP more accurately predicts the diurnal warming than $\mathrm{KC}$. The $\mathrm{KC}$ model generally underestimates the diurnal warming in the near-surface ocean, likely due to prescribed minimum background mixing levels (greater than molecular) in the model that are too large. Standard background level in the $\mathrm{KC}$ model is $1 \times 10^{-5} \mathrm{~m}^{2} / \mathrm{s}$. This was noted by Clayson and Bogdanoff (2013) and Clayson et al. (2016a) in their use of the model to explore diurnal warming. Under strong warming conditions, the convective sublayer at the surface of the ocean may be completely suppressed (Soloviev and Lukas, 2006, p. 267), 

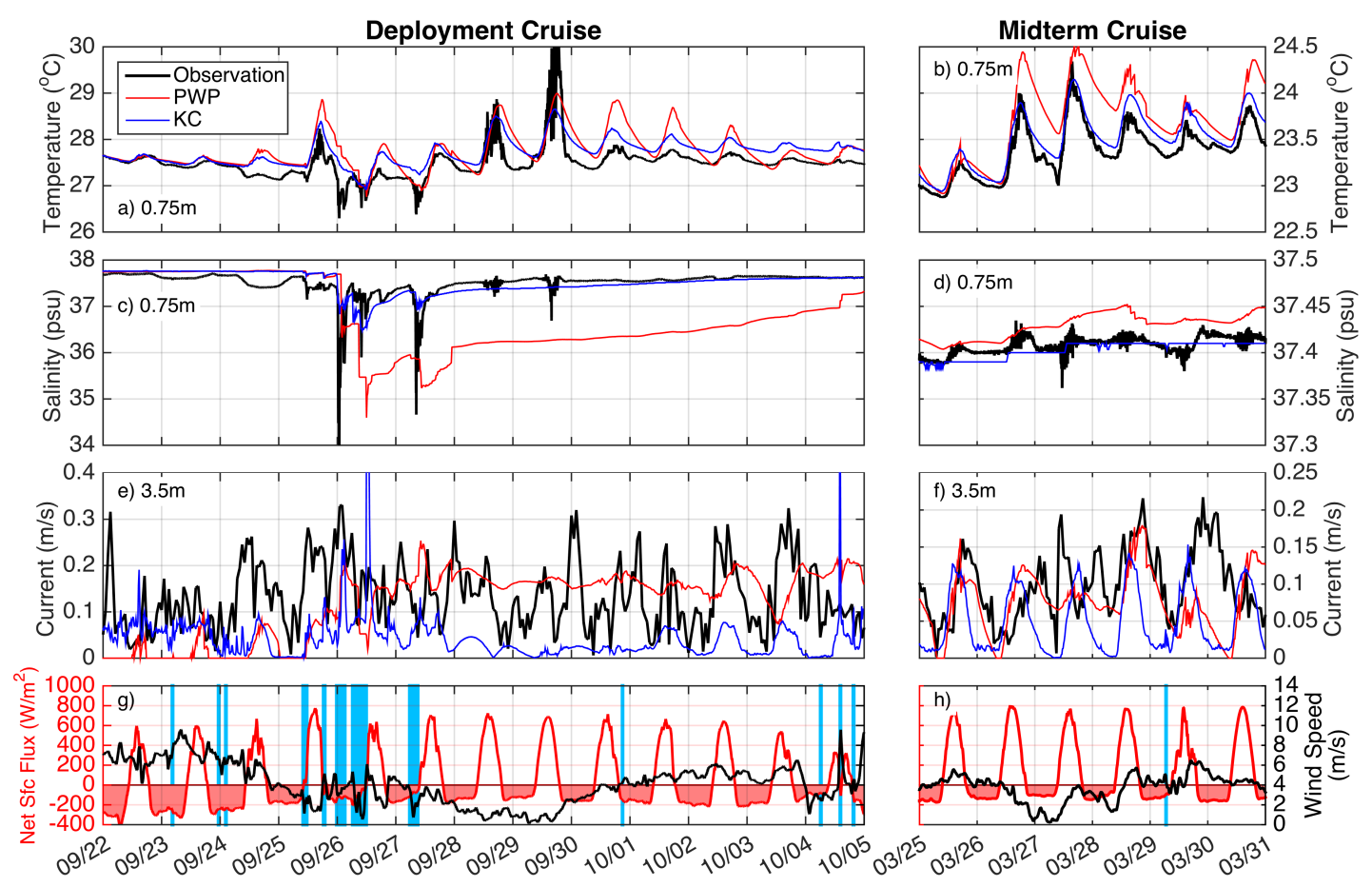

Figure 2-6: The modeled $(a, b)$ temperature, $(c, d)$ salinity, and $(e, f)$ current speed from the Kantha-Clayson (KC, blue lines) and Price-Weller-Pinkel (PWP, red lines) models with observations from the mooring (black line). The shallowest observation is compared to the model estimate at that depth, which is $0.75 \mathrm{~m}$ for temperature and salinity, and $3.5 \mathrm{~m}$ for currents (referenced to the current at $50 \mathrm{~m}$ ). The meteorological conditions at the SPURS mooring $(\mathrm{g}, \mathrm{h})$ with the net surface heat flux in red, wind speed in black, and times of active precipitation in light blue during the deployment (left-side, 9/15/2012 - 10/5/2012) and midterm (right-side, 3/25/2013 - 4/6/2013) cruises.

and turbulent mixing shuts down (also considering a calm wave field). As such, background mixing levels here in $\mathrm{KC}$ are reduced to molecular levels in the upper $5 \mathrm{~m}$ of the ocean when the Richardson number is greater than 0.7 throughout the study.

While reducing the turbulent diffusivity in the near-surface does allow the $\mathrm{KC}$ model to better reproduce the amplitude of the diurnal warming, there was no substantial change in the magnitude of TKE dissipation. A detailed discussion of the differences due to changing the background diffusivity is outside the scope of this study. Here, mixing refers to a turbulent (or molecular) diffusivity, and not TKE or TKE dissipation. Although a turbulent diffusivity can be related to TKE dissipation (Osborn, 1980; St. Laurent and Schmitt, 1999), it requires several assumptions that may not be appropriate for the stable boundary layer. Additionally, the models do not reproduce the high frequency variability in SST (with time 
periods on the order of an hour, for example Fig. 2-6a,c on $9 / 28$ and $9 / 29 / 2012$ ), which are likely due to internal waves. Kelvin-Helmholtz instability (due to relatively strong shear in a stratified fluid) has been shown to be an important source of mixing under stable surface heat flux conditions in the near-surface ocean (Thorpe, 1978).

From $9 / 25-9 / 27 / 2012,9.29 \mathrm{~cm}$ (3.66 in) of rain fell at the mooring. The precipitation event associated with Tropical System Nadine created an intense freshwater lens at the SPURS-I mooring. Differences between KC and PWP are present in salinity as well (Fig. 2-6c), with KC too quickly mixing the freshwater and PWP taking days longer than the observed adjustment of near-surface salinity. Salinity variability from 9/28 - 9/29/2012 is dominated by high frequency oscillations during the daytime, likely due to internal waves (Soloviev et al., 2015), and also observed during the midterm cruise (Fig. 2-6d). The differences between the salinity observations and models from $9 / 22-9 / 25 / 2012$ are due to horizontal advection. A one-dimensional salt budget during that time period reveals large changes not consistent with surface forcing. One-dimensional models are not expected to reproduce horizontal advection.

The modeled current at $3.5 \mathrm{~m}$ is compared to the $3.5 \mathrm{~m}$ observation by subtracting out the observed current at $50 \mathrm{~m}$ to remove a depth-independent current (Fig 2-6e,f). The models better reproduce the observations of current speed during the midterm cruise (Fig. 2-6f) than during the deployment cruise (Fig. 2-6e). While both models reproduce a diurnal warm layer shear evident in the daytime acceleration of the surface current (also shown by enhanced shear in the near-surface during the daytime in Fig. 2-8h,i), the largest modeled shear is generally above the nearest surface current observation of 3.5 meters. Without near-surface observations any discussion of the model differences would be pure speculation. However, neither model reproduces the high frequency (roughly hourly and faster) variability which is present throughout the cruise periods, but specially so during the deployment cruise. A geostrophic current during was present during the midterm cruise time period due to a persistent eddy, but subtracted out by referencing the $50 \mathrm{~m}$ current. Model runs including the geostrophic current were done, but did not change the values of near-surface TKE dissipation enough to explain the observed enhanced TKE dissipation.

The dissipation estimates from the models are compared to the glider observations in Fig. 2-7c-h. Both models reproduce the nocturnal convective signal in TKE dissipation (there is good agreement during the nighttime in Fig. 2-7 between the models and observa- 

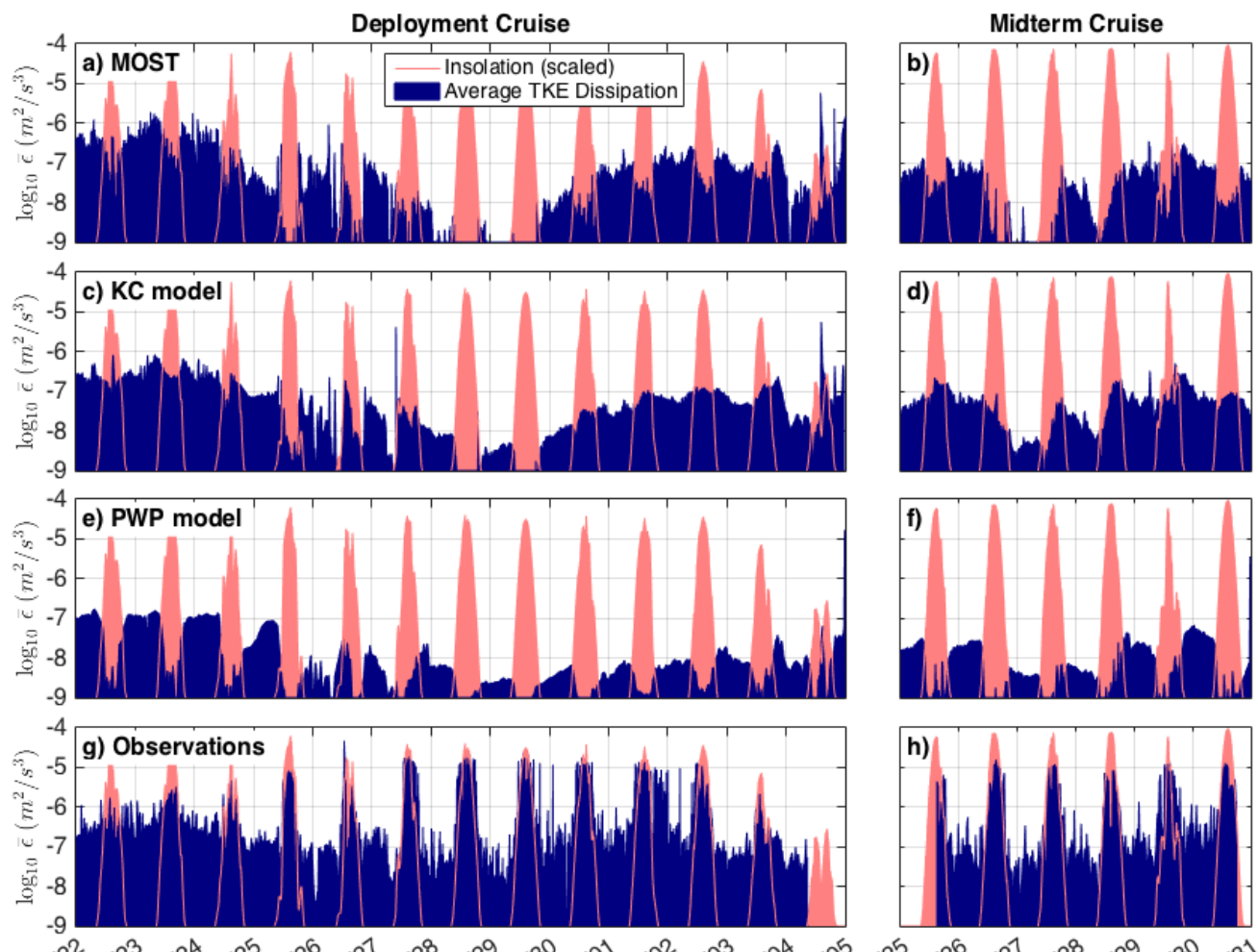

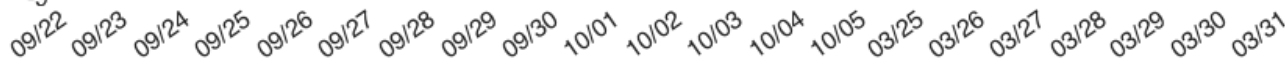

Figure 2-7: Depth-averaged turbulent kinetic energy dissipation over the upper 30 meters from $(\mathrm{a}, \mathrm{b})$ Monin-Obukhov Similarity Theory (MOST) , (c,d) the Kantha-Clayson (KC) model, (e,f) the Price-Weller-Pinkel (PWP) model, and $(\mathrm{g}, \mathrm{h})$ microstructure observations for the deployment (left-side, 9/15/2012 - 10/5/2012) and midterm (right-side, 3/25/2013 $4 / 6 / 2013)$ cruises. The red dashed line and filled curve on all plots is scaled incoming solar radiation to show times when the sun is up.

tions). At the beginning of the deployment cruise record until $9 / 25 / 2012, \mathrm{KC}$ does well at reproducing TKE dissipation under the windy conditions (Fig. 2-7c) however, it does miss the small enhancement during the daytime. PWP and KC have quite different estimates of dissipation during the daytime, but neither model reproduces the observed daytime enhancement. As previously noted, PWP does not explicitly calculate TKE dissipation and there is no exact method for obtaining an estimate of TKE dissipation. The lack of reproduction of the daytime dissipation by $\mathrm{KC}$ may indicate that the model is not properly reproducing the near-surface shear.

The differences in the TKE dissipation models become clearer by examining the models in depth and time (Fig. 2-8). Both models have a warmer than observed upper ocean at the end of the model run (Fig. 2-8a,b,c), which is due to a sharp drop in the observed heat content on 9/26/2012 associated with advection and the large precipitation event associated 
with Nadine. KC better reproduces the near-surface ocean stratification ( $N^{2}$, Fig. 2-8d,e), but does not have a warm enough sea surface (Fig. 2-6a). PWP suffers from a higher than observed stratification due to the long residence time of the salinity anomaly associated with the large precipitation event. Neither model reproduces the 5-10 meter enhanced shear during the daytime with much of the modeled shear confined to the near-surface region without observations ( $S^{2}$, Fig. 2-8g,h,i).

The stability of the mean shear is characterized by reduced shear, $S_{r e d}^{2}=S^{2}-4 N^{2}$, which when greater than zero indicates a gradient Richardson number $(R i)$ less than 0.25. Wenegrat and McPhaden (2015) note that reduced shear is preferable to $R i$ because it linearizes observational error (by adding shear and buoyancy, rather than a fraction) and its distribution is less skewed. The reduced shear estimates for the PWP model (Fig. 2-81) should always be less than or equal to zero, as the model imposes that a profile must be characterized by a stable $R i$; the green regions indicate areas where the model completely mixes the water column and reduced shear is approximately zero. KC (Fig. 2-8k) misses the deep unstable layer during the first part of the deployment cruise (green and yellow in Fig. 2-8j). Additionally, the unstable layers are shallower in the model than observed. So, while the models do reproduce important characteristics of temperature, salinity and currents of the near-surface, the vertical structure has substantial unresolved differences from the observations.

Breaking surface gravity waves and Langmuir circulation are potentially important sources of turbulence in the upper ocean (Kitaigorodskii et al., 1983; Leibovich, 1983; Weller and Price, 1988; Craig and Banner, 1994; McWilliams et al., 1997). An updated version of the KC model (Kantha and Clayson, 2004) includes a parameterization of both breaking surface wave and Langmuir turbulence. Model simulations were performed with the inclusion of the breaking wave and Langmuir turbulence parameterizations over a broad range of oceanic wave and atmospheric conditions. Although notable differences in the upper ocean current and density profiles were evident as compared to the basic model, none of these differences resulted in enhancing the shear and dissipation to observed values. In addition, there were several near-calm days (such a 9/28 and 9/29/2012) during the SPURS-I field campaign where the ocean surface was glass calm and no breaking waves were observed.

In general, the $\mathrm{KC}$ model estimates the surface temperature and salinity outside of large precipitation events well, and properly resolves the mixing levels. The PWP model gen- 


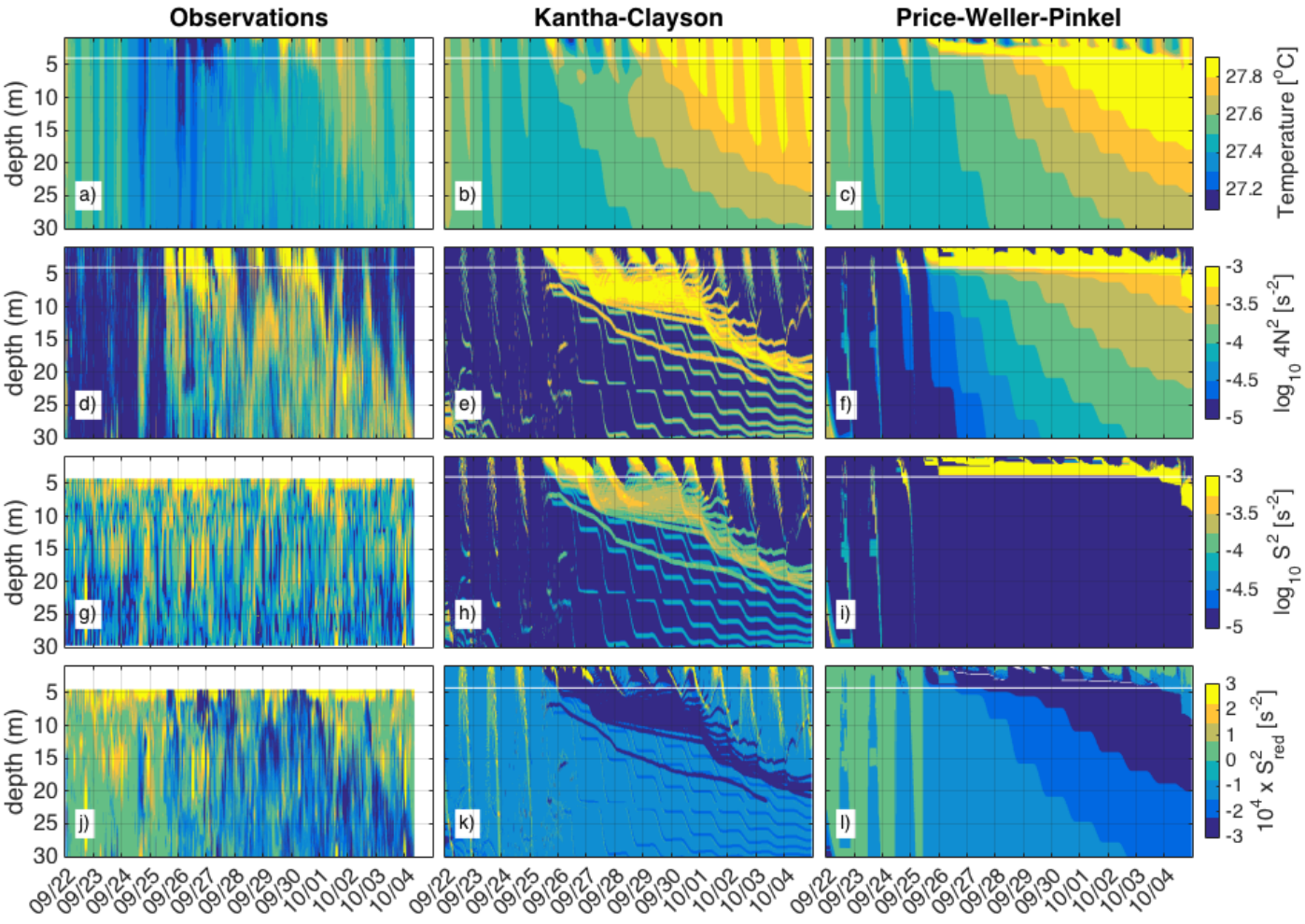

Figure 2-8: Observations (left), Kantha-Clayson model results (middle), and Price-WellerPrinkel model results (right) of (a,b,c) temperature, (d,e,f) buoyancy frequency, (g,h,i) shear squared, and $(\mathrm{j}, \mathrm{k}, \mathrm{l})$ reduced shear $\left(S^{2}-4 N^{2}>0\right.$ indicates flow with a $\left.\mathrm{Ri}<0.25\right)$ from the deployment cruise time period in which the glider was profiler near the central mooring $(9 / 22 / 2012-10 / 5 / 2012)$. The white line indicates 4 meters, which is the minimum depth of the shear observations. Note the high shear below 4 meters that is not simulated by the models, as the shear during the daytime is confined above the white line in the models. 
erates better estimates of the large diurnal warming events, but overestimates the weaker events. Neither model reproduces the large observed TKE dissipation during the daytime. The disagreement in dissipation between the models and the observations appears to be due to a misprediction of shear. Therefore, either both models are incorrect in their calculation of shear production (produced by differing methods between the models), or the shear production mechanism is a physical process not present in either model.

\subsubsection{Monin-Obukhov Similarity Theory}

A third method for estimating turbulent characteristics in the surface layer is MoninObukhov Similarity Theory (MOST; Monin and Obukhov, 1954) and estimates turbulent characteristics of the boundary layer from surface forcing. MOST scales the turbulent conditions of the atmospheric and oceanic boundary layers as a function of surface forcing, distance from the surface (positive value), $z$, and a length scale, $L_{o}$, the Obukhov length. While the theory was developed for the atmosphere, it has been used for the ocean (e.g., Large et al., 1994; McPhee, 1994; Tedford et al., 2014; Callaghan et al., 2014). It is wellstudied under unstable conditions in the atmosphere, but less so for stable conditions and the ocean. Although MOST is contained within some of the closure schemes of the KC model, it is a useful exercise to examine the similarity theory in its purest form.

Following Wyngaard et al. (1971) and Fairall et al. (1980), the turbulent kinetic energy dissipation rate can be written as a function of the stability parameter $(\zeta)$, defined as

$$
\zeta=\frac{z}{L_{o}}
$$

and the Obukhov length is defined as

$$
L_{o}=\frac{u_{*}^{3}}{\kappa B_{0}}
$$

where $u_{*}$ is the surface frictional velocity (the wind stress, $\tau=\rho_{0} u_{*}^{2}$ ), $\kappa$ is the von Karman constant $(\kappa=0.41)$, and $B_{0}$ is the surface buoyancy flux. During the daytime a positive net heat flux leads to a warming ocean with a positive buoyancy flux (a decrease in mass or 
density). The surface buoyancy flux can be calculated following Gill (1982) as

$$
B_{0}=\frac{g \alpha}{\rho_{o} c_{p}} Q_{N}-g \beta S_{o}(E-P)=\frac{g \alpha}{\rho_{o} c_{p}} Q_{N}-g \beta S_{o}\left(-\frac{Q_{L}}{\rho_{0} L_{v}}-P\right),
$$

where $g$ is the gravitational constant, $\alpha$ is the thermal expansion coefficient of sea water $\left(-\rho^{-1} \partial \rho / \partial T\right.$, generally $\left.>0\right), c_{p}$ is the specific heat of seawater, $Q_{N}$ is the net surface flux into the ocean (Eq. 4.1), $\beta$ is the saline contraction coefficient of seawater $\left(\rho^{-1} \partial \rho / \partial S>0\right)$, $S_{o}$ is the surface salinity, $E$ is evaporation $(\mathrm{m} / \mathrm{s}), P$ is precipitation $(\mathrm{m} / \mathrm{s}), Q_{L}$ is the latent heat flux, and $L_{v}$ is the latent heat of vaporization of sea water. The extinction profile of solar radiation is neglected within the net surface flux of MOST, and is likely a significant source of error for the theory in the ocean during the daytime. MOST-estimated TKE dissipation is

$$
\epsilon_{M O}=\frac{u_{*}^{3}}{\kappa z} \phi_{\epsilon}(\zeta)
$$

where $\phi_{\epsilon}$ is the universal MOST function for dissipation of Edson and Fairall (1998), given as:

$$
\begin{aligned}
& \phi_{\epsilon}(\zeta)=\left(1+0.5|\zeta|^{2 / 3}\right)^{3 / 2}, \quad \text { for } \zeta<0 \\
& \phi_{\epsilon}(\zeta)=\left(1+2.5|\zeta|^{2 / 3}\right)^{3 / 2}, \quad \text { for } \zeta \geq 0
\end{aligned}
$$

MOST does not reproduce the observed high daytime dissipation (Fig. 2-7a,b), but it does compare well with the observations under windy conditions and under most conditions with the KC model. To compare MOST to observations, a buoyancy Reynolds number $\left(R e_{b}=\epsilon / \nu N^{2}\right)$ is used in order to take varying stratification into account. $R e_{b}$ defines the scale separation between the range of scales over which background shear and stratification can act. $R e_{b}^{3 / 4}$ is the intermittency factor (Thorpe, 2005), which is the ratio of the Ozmidov (largest eddies) length scale to the Kolmogorov (dissipative/smallest) length scale. Isotropy is expected when there is enough scale separation between the largest and smallest eddies, corresponding to $R e_{b}>200$ (Gargett et al., 1984). The buoyancy Reynolds number is calculated using the glider data averaged over the upper 10m, and compared to a buoyancy Reynolds number calculated using MOST estimated TKE dissipation $\left(R e_{b-M O}\right)$ averaged over the upper 10 meters (Fig. 2-9).

If MOST properly predicts dissipation, the data should scatter around the 1:1 line. 
a) Stablizing Surface Forcing $\left(Q_{\text {net }}>0\right)$

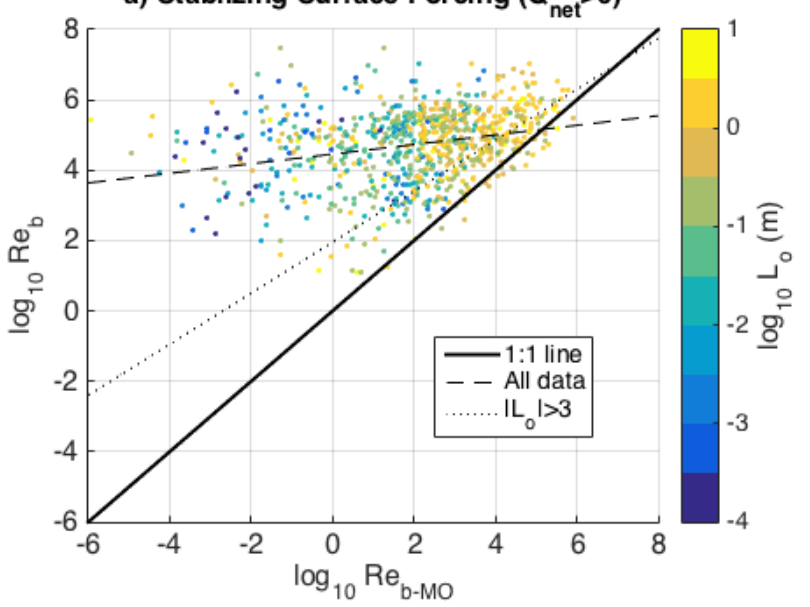

b) Destablizing Surface Forcing $\left(Q_{\text {net }}<0\right)$

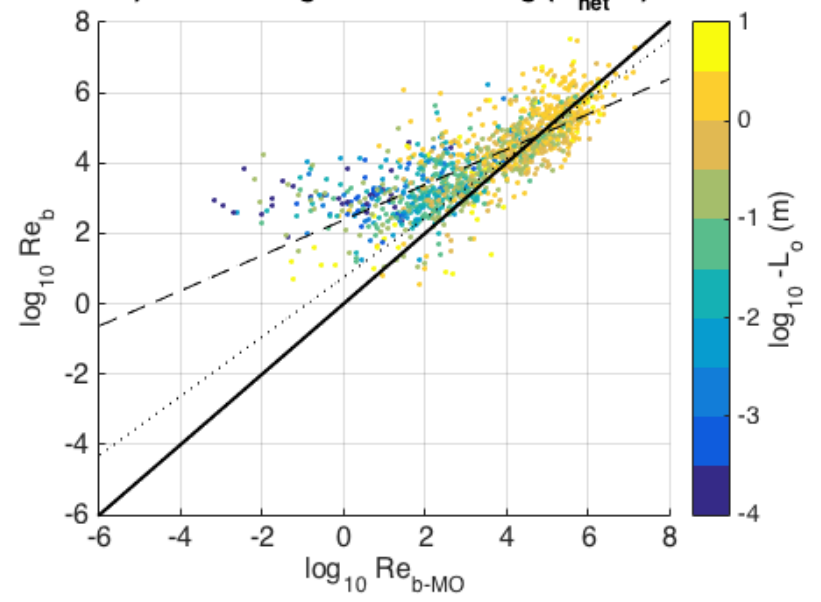

Figure 2-9: Scatter plot of the buoyancy Reynolds number $\left(\mathrm{Re}_{\mathrm{b}}\right)$ calculated from the glider dissipation and a buoyancy Reynolds number for MOST ( $\mathrm{Re}_{\mathrm{b}-\mathrm{MO}}$ ) under (a) stabilizing surface forcing and (b) destabilizing surface forcing (b) averaged over the upper 10 meters of the ocean. The color indicates the absolute value of Obukhov length, and it is positive in (a) for stable forcing, and negative in (b) for destabilizing forcing. The dashed line is the best fit for all of the data in each regime. The dotted line is the best fit for data with $\left|\mathrm{L}_{o}\right|>3$, corresponding to the largest color bar interval and roughly half the depth over which the data is averaged. Note the drop off at small Obukhov lengths for destabilizing surface forcing and large scatter for stabilizing surface forcing. 
However, a drop-off at low Obukhov length may be expected as Foken (2006) states that MOST is only valid when $|\zeta| \leq 1-2$, which would be $\left|L_{o}\right| \sim O(5 \mathrm{~m})$ since the data are an average of the upper $10 \mathrm{~m}$. Here, a cutoff of $3 \mathrm{~m}$ is used as $\log _{10}(3)$ is roughly 0.5 corresponding to the largest color bar interval in Fig. 2-9.

Under destabilizing surface forcing (Fig. 2-9b), the correlation coefficient between the logarithm of $R e_{b}$ and $R e_{b-M O}$ is 0.77 for all data with $\left|L_{o}\right|>3 \mathrm{~m}$. Both best fit lines are statistically significant and explain $60 \%$ of the variance of $R e_{b}$, using an analysis of variance technique. The standard error is 0.04 and 0.02 for all data and for data with $\left|L_{o}\right|>$ $3 \mathrm{~m}$, respectively. The best fit line for all destabilizing surface forcing data is statistically significantly different from the 1:1 line at the $95 \%$ confidence level; however, the best fit line for data with $\left|L_{o}\right|>3 \mathrm{~m}$ is not statistically different from the 1:1 line. Therefore, for the cases when MOST is expected to be valid under destabilizing conditions $\left(\left|L_{o}\right|>3 \mathrm{~m}\right)$, the prediction of $R e_{b-M O}$, and thus the prediction by MOST is not statistically different from the observations (MOST is a good predictor under those conditions).

For stabilizing surface forcing (Fig. 2-9a), the linear correlation coefficient between the logarithm of $R e_{b}$ and $R e_{b-M O}$ is 0.27 for all data and 0.71 for data with $\left|L_{o}\right|>3 \mathrm{~m}$. Both are statistically significant at the $95 \%$ confidence level. For all stabilizing surface forcing data, the best fit line is statistically significant, but explains only $7 \%$ of the variance of $R e_{b}$; the standard error is 0.12 . Parsing just the data with $\left|L_{o}\right|>3 \mathrm{~m}$, the best fit line is also statistically significant, and explains $71 \%$ of the variance with a standard error of the fit of 0.08. The standard error is four times greater for the stabilizing best fits than for the destabilizing fits. The fit for data under conditions in which MOST is expected to be valid has a substantially increased explanation of variance and reduction in standard error. However, both lines are statistically significantly different from the 1:1 line at the 95\% confidence level. Even when MOST is expected to be valid where $\left|L_{o}\right|>3 \mathrm{~m}$, the prediction of $R_{b-M O}$, and thus MOST-estimated TKE dissipation is statistically significantly different from the observations.

It is important to note that Smyth and Moum (2000) caution that errors in TKE dissipation due to anisotropy that are then used to calculate $R e_{b}$ to analyze whether a flow is isotropic can provide an incorrect outcome (flow is considered anisotropic with $R e_{b}<200$ ). This may explain some of the scatter of values with large Obukhov length at low $R e_{b}$ under destabilizing conditions. At sufficiently large $R e_{b}$ the possible errors due to anisotropy likely 
do not explain the scatter, (as observed in destabilizing conditions, Fig. 2-9b) as the range of errors will still provide a value well within the isotropic regime of buoyancy Reynolds numbers.

Under very stable conditions $(\zeta>>1)$ the applicability of MOST in the atmospheric boundary layer has been brought into question (Pahlow et al., 2001; Kouznetsov and Zilitinkevich, 2010; Grachev et al., 2013). The results here echo the sentiment that MOST is likely not applicable under stabilizing surface forcing conditions. Under these conditions, 'z-less turbulence' is expected, meaning that the distance from the surface is no longer important, and turbulence is limited by the stratification (Monin et al., 1971; Wyngaard and Coté, 1972; Pahlow et al., 2001). Therefore, the turbulence is no longer directly determined by the surface forcing. In addition, MOST does not take into account penetrative solar radiation, which in the strongly stable upper ocean is a dominant factor in predicting the upper ocean temperature structure (Gentemann et al., 2009).

The influence of internal waves, as observed during the daytime in the near-surface ocean (e.g. Thorpe, 1978), may explain the inability of surface-forcing based models to predict appropriate levels of turbulence. A recent study of a lake by Tedford et al. (2014) found MOST does not work under low wind speed conditions as high-frequency internal waves augment the turbulence in the near-surface, specifically propagating along the diurnal thermocline and breaking. Cheng et al. (2005) found that MOST does not properly capture turbulence in the stable boundary layer during Kelvin-Helmholtz instability as the surface forcing cannot properly define the turbulence of the flow. Specifically, they note that strong and intermittent turbulence associated with the breaking waves was not captured by MOST, but the theory does appear to be applicable in the presence of a non-breaking wave.

While MOST is a good predictor of the near-surface TKE dissipation during destabilizing surface forcing with sufficiently large Obukhov length, under stable conditions it does not appropriately predict appropriate turbulence levels. The influence of internal waves on the turbulence of daytime near-surface ocean is the focus of Chapter 3. In addition, since MOST uses the instantaneous surface forcing to predict turbulent parameters (it assumes that turbulence can be predicted by current conditions), phenomena such as the diurnal warm layer shear, which are caused by time-integrated surface forcing cannot be predicted, and need to be explored separately. 


\subsection{Diurnal warm layer shear}

The diurnal warm layer shear (hereafter DWL shear) is caused by momentum trapped by the stable stratification of diurnal warming during the daytime in the near-surface ocean. This momentum trapping has been observed in several studies and referred to by several names, including slippery seas (Houghton, 1969), a slippery near-surface layer (Kudryavtsev and Soloviev, 1990), and the diurnal jet (Price et al., 1986). Similar near-surface layers can be caused by advection, precipitation, or runoff. Under conditions of diurnal warming, momentum transferred from the atmosphere to the ocean can remain trapped until the net surface heat flux goes negative, where nocturnal convection ensues, or until another mixing event causes the descent of the DWL shear (Smyth et al., 2013). Under strong diurnal warming, this thin shear layer is confined to the very near-surface (Woods, 1968; Soloviev and Lukas, 2006).

This phenomenon is quite difficult to observe as it occurs in the very near-surface (0-3 $\mathrm{m}$ ), so beyond very few specific observational campaigns such as Kudryavtsev and Soloviev (1990), modeling augments our understanding of this phenomenon. A large-eddy simulation described in Wang (2001) does show elevated TKE dissipation levels in the near-surface ocean during the daytime likely due to the diurnal warm layer shear. The modeled elevated levels are orders of magnitude smaller than the observations discussed here, $O\left(10^{-7}\right) \mathrm{W} / \mathrm{kg}$ vs. $O\left(10^{-5}\right) \mathrm{W} / \mathrm{kg}$, respectively. DWL shear is reproduced by both KC and PWP (any model that similarly mixes heat and momentum will have a DWL shear), but the models do not reproduce the observed dissipation levels as shown in the previous section.

Shear observations of the upper ocean are composited from the nearest-surface ADCP during the same time period of observations of TKE dissipation from the gliders. Composite averages are shown in Fig. 2-10 for wind speeds less than $5 \mathrm{~m} / \mathrm{s}$ for day and night, and wind speeds greater than $5 \mathrm{~m} / \mathrm{s}$. High shear is observed near the surface under all conditions. Larger values of shear are expected near the surface under wind-driven mixing. This enhancement is observed during night, light wind conditions (Fig 2-10a) and windy conditions (Fig 2-10c). In addition, there is enhanced shear observed at night associated with high stratification and light wind conditions, which can be attributed to weak convection allowing the daytime stratification to persist into the night.

Near the surface during the daytime under low wind speed conditions, shear increases 

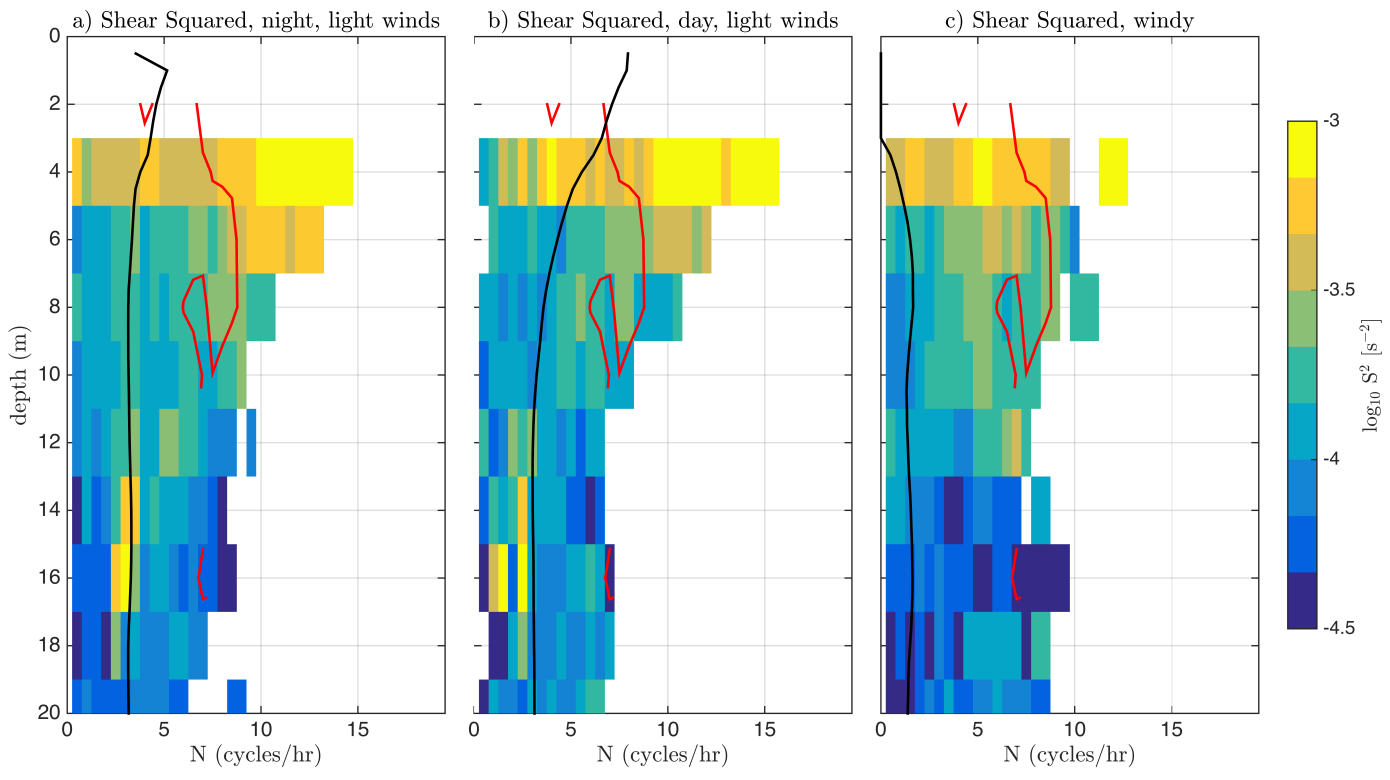

Figure 2-10: Similar to Fig. 2-5, but composite of average shear squared shown by color shading binned by depth and buoyancy frequency (cph) for (a) nighttime light wind conditions $\left(\mathrm{Q}_{\text {net }}<0\right.$, wspd $<5 \mathrm{~m} / \mathrm{s}$ ), (b) daytime light wind conditions $\left(\mathrm{Q}_{\text {net }}>0\right.$, wspd $<5$ $\mathrm{m} / \mathrm{s}$ ), and (c) windy conditions (wspd $>5 \mathrm{~m} / \mathrm{s}$ ). The black lines indicate the average buoyancy frequency profile for each condition. The red contour shows where the ocean warms, on average, more than $0.25^{\circ} \mathrm{C}$ from the value at sunrise at each depth, based on the light wind daytime composite, and is plotted on all three for reference. 
with increasing buoyancy frequency (Fig 2-10b). The expected maximum current due to trapped momentum in the layer is $0.1-0.3 \mathrm{~m} / \mathrm{s}$ (Price et al., 1986; Kudryavtsev and Soloviev, 1990; Soloviev and Lukas, 2006); this does not include background shear of the mean ocean current. For reference, a $0.1 \mathrm{~m} / \mathrm{s}$ current change over $2 \mathrm{~m}$ (the difference in the depth bins of the ADCP) is a shear squared of $2.5 \times 10^{-3} \mathrm{~s}^{-2}$ and $0.3 \mathrm{~m} / \mathrm{s}$ current change over $2 \mathrm{~m}$ is $2.25 \times 10^{-2} \mathrm{~s}^{-2}$. These magnitudes compare with observations of shear squared during the strongest stratification observed nearest the surface.

A wind threshold of $2 \mathrm{~m} / \mathrm{s}$ appears to be a cutoff between different regimes of diurnal warming (Webster et al., 1996; Soloviev and Lukas, 1997). To explore possible regime differences, the SPURS-I data were separated into wind speeds less than $2 \mathrm{~m} / \mathrm{s}$ and between 2 and $5 \mathrm{~m} / \mathrm{s}$ however, differences in the regimes were not apparent. This is likely due to the minimum depth of the ADCP being greater than the strongest temperature gradient confined to the upper 1 meter of the ocean (Soloviev and Lukas, 1997). Although the nearestsurface currents are not observed, enhanced daytime shear is observed and it increases as stratification increases. If the DWL shear is responsible for the enhanced TKE dissipation, a mechanism for shear production is necessary to convert the mean kinetic energy to TKE that can be dissipated. As shear and stratification increase, the stability of the current can change, become unstable, and overturn. This is the self-regulating diurnal thermocline.

\subsubsection{Self-regulating diurnal thermocline theory}

Price et al. (1986) detail the diurnal cycle and the concept of a self-regulating diurnal thermocline, a mechanism for shear instability. Soloviev and Lukas (2006, §4.2.2) expand on this theory using the concept of a slab mixed layer depth proportional to the Obukhov length $\left(\mathrm{L}_{o}\right)$. Within this construct, the layer has a constant temperature and velocity at the surface, and is forced from the surface. Ignoring the effects of salinity (in the calculation of the Obukhov length as well), the temperature change in a slab layer with time $(\Delta T)$ can be approximated as $\Delta T \sim Q_{N} t /\left(\rho_{0} c_{p} L_{o}\right)$, where $t$ is time. If all the wind stress accelerates the layer, the change in velocity with time $(\Delta u)$ can be written as $\Delta u \sim u_{*}^{2} t / L_{o}$. Soloviev and Lukas (2006) define a bulk Richardson number by assuming diurnal warm layer shear exists to a depth $\Delta H$ below the slab layer, as $R i_{S L} \sim \Delta H /\left(\kappa u_{*} t\right)$.

To explore the stability of the observed mean current, a composite of reduced shear for the upper ocean over a range of buoyancy frequencies is calculated (Fig. 2-11). A buoyancy 

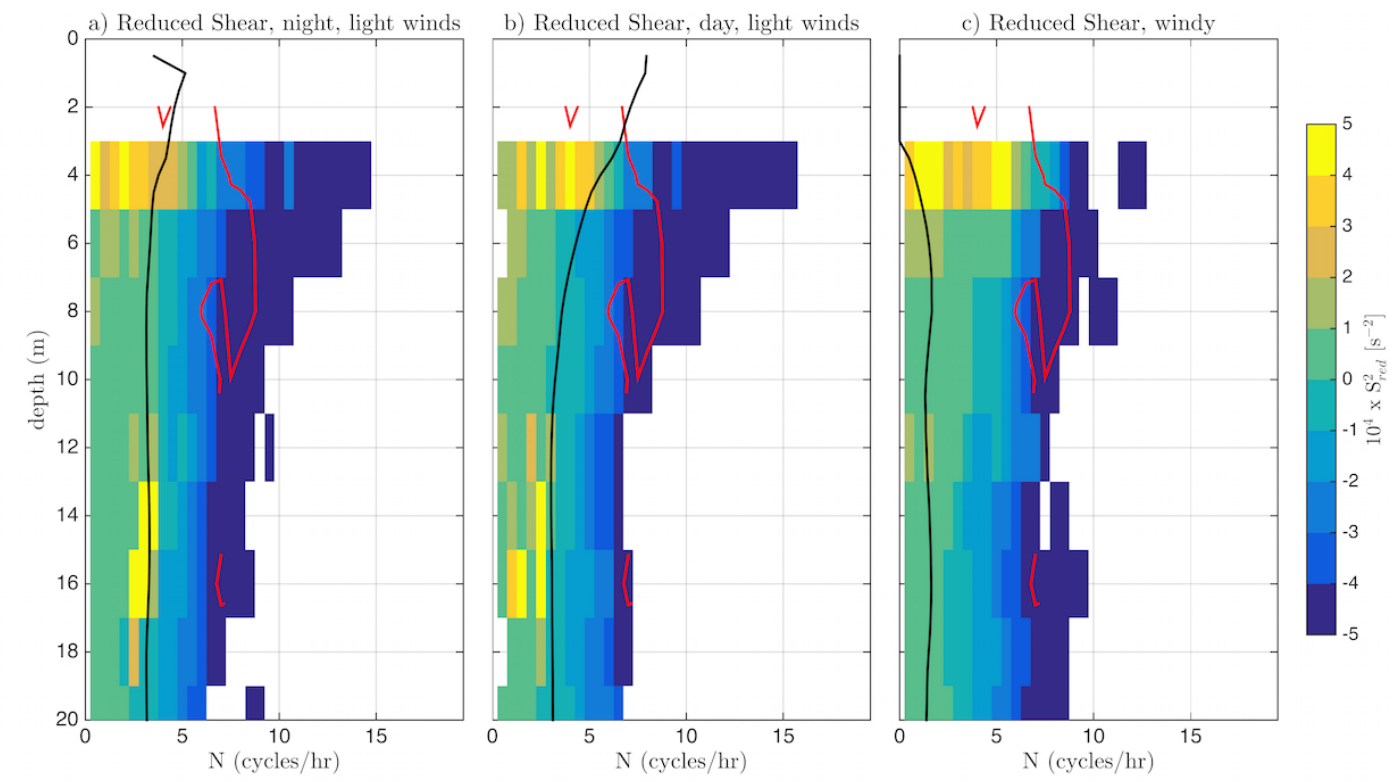

Figure 2-11: Similar to Fig. 2-5 and 2-10, but composite of average reduced shear $\left(\mathrm{S}^{2}-4 \mathrm{~N}^{2}\right.$, where values greater than 0 indicate $\mathrm{Ri}<0.25$ corresponding to unstable Kelvin-Helmholtz conditions) shown by color shading binned by depth and buoyancy frequency (cph) for (a) nighttime light wind conditions $\left(\mathrm{Q}_{\text {net }}<0\right.$, wspd $<5 \mathrm{~m} / \mathrm{s}$ ), (b) daytime light wind conditions $\left(\mathrm{Q}_{\text {net }}>0\right.$, wspd $<5 \mathrm{~m} / \mathrm{s}$ ), and (c) windy conditions (wspd $>5 \mathrm{~m} / \mathrm{s}$ ). The black lines indicate the average buoyancy frequency profile for each condition. The red contour shows where the ocean warms, on average, more than $0.25^{\circ} \mathrm{C}$ from the value at sunrise at each depth, based on the light wind daytime composite, and is plotted on all three for reference.

frequency from the mooring and the shear squared in Fig. 2-10 are used for the same regimes: wind speeds less than $5 \mathrm{~m} / \mathrm{s}$ for day and night, and wind speeds greater than $5 \mathrm{~m} / \mathrm{s}$. The blue regions are, on average, stable to Kelvin-Helmholtz (K-H) instability. Under nighttime light wind (Fig. 2-11a) and windy conditions (Fig. 2-11c), the mean buoyancy profile (black line) is associated with reduced shear greater than zero (unstable to $\mathrm{K}-\mathrm{H}$ ). The average daytime profile (Fig. 2-11b) is marginally stable.

Reduced shear is less than zero where diurnal warming is observed during the daytime. Average warming greater than $0.25^{\circ} \mathrm{C}$ is shown in the area above and to the right of the red line on Fig. 2-11b. Even though enhanced shear is observed in the near-surface ocean (Fig. 2-10b), the buoyancy suppression under the high stratification is greater than the shear production (since reduced shear is greater than zero) and is, on average, dynamically stable. Although diurnal warming is associated with a $R i$ stable regime, it does not preclude overturning. Diurnal shear layers have been shown to be marginally stable (Kudryavtsev 
and Soloviev, 1990), and overturning is expected based on self-regulating diurnal thermocline theory. However, the observations here indicate, on average, a statically stable diurnal warm layer. Under conditions of relatively strong warming, the marginally stable layer may be above the shallowest observations from SPURS-I. During the diurnal peak in observed TKE dissipation (4 hour period in the middle of the day) on the $9 / 27-9 / 29 / 2012$ and $3 / 25$ $3 / 31 / 2013$, the near-surface buoyancy frequency remains above 5 cycles/hr and dynamically stable for all days.

A canonical diurnal cycle of the near-surface is created to explore conditions under diurnal warming (Fig. 2-12) using hourly averages. The daily averaged wind speed was less than $5 \mathrm{~m} / \mathrm{s}$ for all 18 days used in the composite. A clear peak in stratification lags the peak warming by about 2 hours, persistent into the nighttime, and is mixed down by convection (Fig. 2-12a). By sunrise, a minimum in stratification is reached as nocturnal convection has been acting to mixed the upper ocean all night. Following the minimum, an increase in stratification is observed shortly after the net surface flux changes sign and the ocean is gaining heat until peak stratification is reached.

The strongest shear in the upper few meters of the ocean corresponds with the strongest stratification ( Fig. 2-11b and Fig. 2-12b). The increased shear and stratification during the daytime are associated with a negative reduced shear, which is dynamically stable (Fig. 212d). The descent of the DWL shear is observed shortly after sunset, and is associated with an increased occurrence of the percent of time that the reduced shear is greater than zero, leading to K-H instability (Fig. 2-12f). The only average unstable conditions are found in the near-surface during the nighttime, associated with convection (Fig. 2-12d), which is delineated by a black contour on all plots. The largest dissipation is found about solar noon (Fig. 2-12e) and clearly between sunrise and sunset. It is offset at an earlier time from the strongest stratification and shear. Since observations of shear are not available above 4 meters, the results here cannot provide the whole story, and the structure of shear near the surface may change dramatically. However, the largest dissipation does not correspond with strongest diurnal shear. In addition, since both KC and PWP resolve enhanced daytime shear, if the DWL shear and self-regulating diurnal thermocline mechanism are the sole cause of the enhanced dissipation some level of enhanced dissipation should be expected in the models. None is observed. 


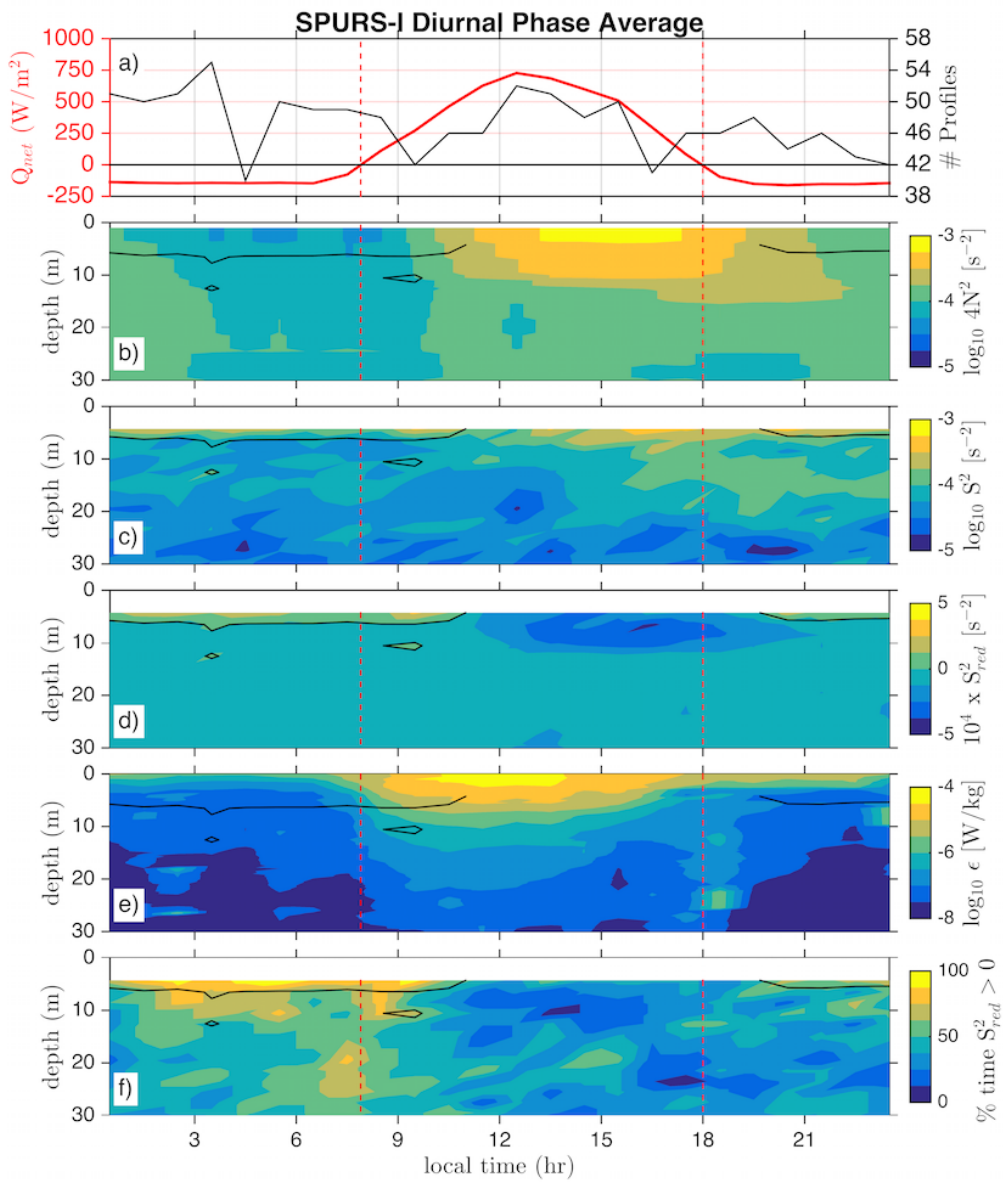

Figure 2-12: Diurnal phase composite of 18 days with diurnal warming and no measurable precipitation during the SPURS-I deployment and midterm cruises. (a) Net surface heat flux average (red line) and the number of glider profiles in each hourly average (black line) for the composited days. Composite diurnal phase average of (b) four times the buoyancy frequency from the mooring, (c) shear squared from the mooring, (d) calculated reduced shear by matching observations of buoyancy frequency nearest the observations of shear in time, (e) TKE dissipation from the gliders, and (f) percent of time the calculated reduced shear is greater than zero, in local time. Vertical red dashed lines indicate time periods of positive and negative net surface heat flux, and the black contour is the zero line of reduced shear (equivalent to a $\mathrm{Ri}$ of 0.25 ). 


\subsubsection{Statistics of shear and stability}

Empirical probability distribution functions (PDFs) of TKE dissipation and reduced shear are used to explored the likelihood of unstable conditions (Fig. 2-13). PDFs are plotted for wind speeds less than $5 \mathrm{~m} / \mathrm{s}$ for day and night, and wind speeds greater than $5 \mathrm{~m} / \mathrm{s}$ in 10 meter depth bins over the upper 30 meters of the ocean. Shay and Gregg (1986) show lognormal distributions of TKE dissipation for a fully turbulent regime. However, none of the distributions of TKE dissipation can be statistically classified as lognormal. This is likely because the flow is not fully turbulent under any of the conditions over the entire depth of the bin. For both TKE dissipation and reduced shear, none of the distributions in each depth bin are statistically from the same populations at the $99 \%$ confidence level using a two-sample Kolmogorov-Smirnov test. This indicates that the three regimes are statistically different.

The statistics of TKE dissipation and reduced shear for each distribution are provided in Tables 2.2 and 2.3, respectively. The error bars are the $95 \%$ confidence interval using a bootstrapping method with 1000 samples. The most striking feature for nighttime conditions is the shift of the center of the distribution from a median of $-7.28 \pm 0.03$ to $-9.10 \pm 0.04$ $\mathrm{W} / \mathrm{kg}$ with a bimodal distribution at intermediate depth. Smyth et al. (1997) show a similar shift in distribution centers due to isolation of a layer from surface forcing. The mean and median of reduced shear is greater than zero for the uppermost bin (Fig. 2-13b), and more skewed than deeper distributions. This indicates the occurrence of stronger shear or weaker stratification closer to the surface, as expected with convection. The mean and median of the deeper bins is negative indicating an increased likelihood of stable conditions with depth.

Under windy conditions, the center of the TKE dissipation distributions shifts less than the other regimes with depth. They have the smallest interquartile ranges (IQR, 3rd quartile minus the 1st quartile) indicating a more condensed distribution about the mean, and are also observed by the relatively smaller standard deviations. The mean of the reduced shear PDFs are positive for the $4-10 \mathrm{~m}$ and $10-20 \mathrm{~m}$ bins, but centered about 0 for the deepest bin (negative median and positive mean). The shape of the distribution is similar to that observed by Wenegrat and McPhaden (2015), skewed with a long tail in stable reduced shear.

In contrast, in the upper bin under daytime light wind conditions the highest occurrence 


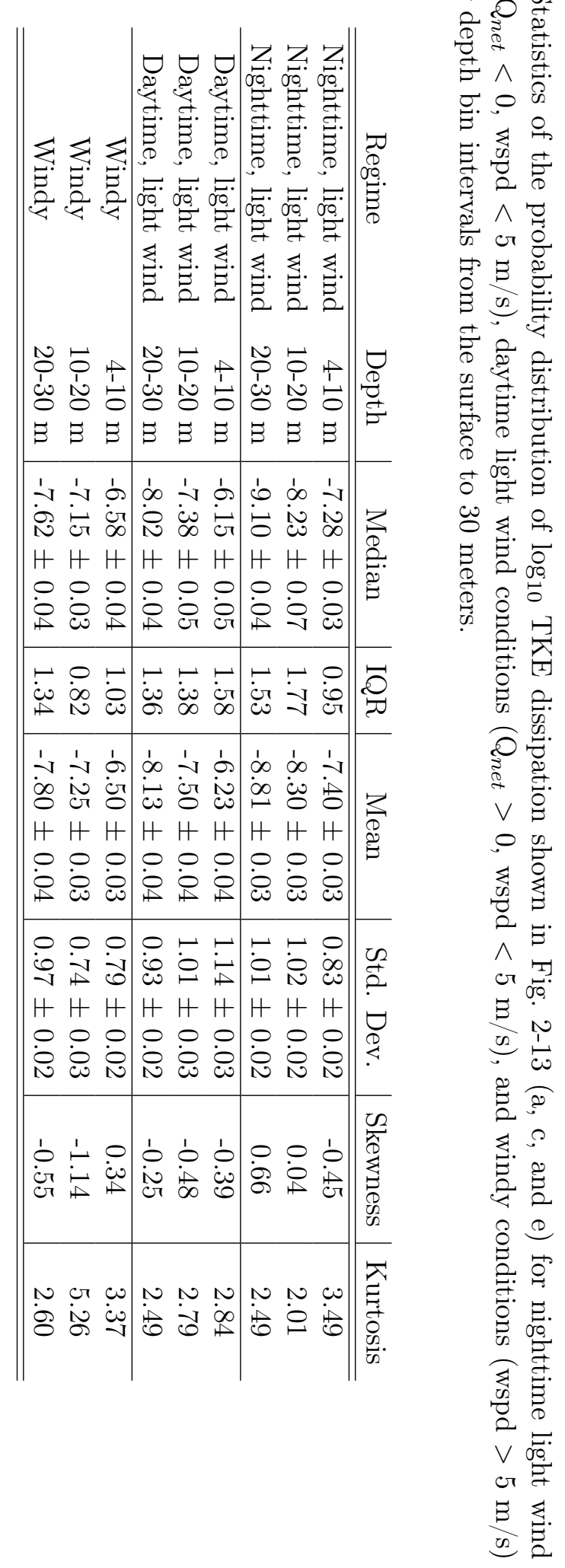

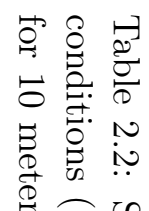

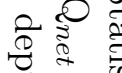

$\therefore \wedge$

. $\approx$ 웅

ह

$\wedge$ ت

F

匹

के

.

E

$+\stackrel{0}{0}$

棺

ट

8

를

(1)

$\checkmark$ 을

○

F

or

.

क $\frac{N}{\sim}$

ใै

$\varangle S$

艺

80

.

응

§

2

$\checkmark \stackrel{0.0}{\circ}$

다 द

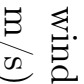




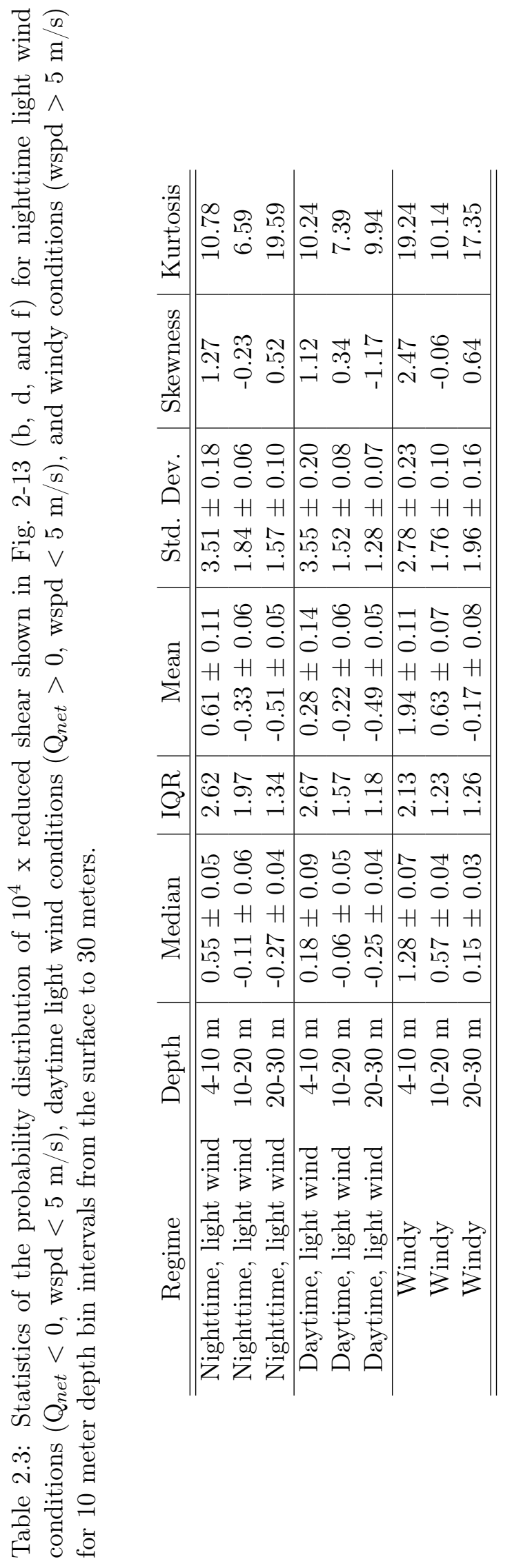


of large TKE dissipation is observed (Fig. 2-13a) in parallel with the highest occurrence of stable reduced shear (Fig. 2-13b). The distribution of dissipation is the least peaked (smallest kurtosis), negatively skewed with a mean of $-6.15 \pm 0.05 \mathrm{~W} / \mathrm{kg}$ indicating a broad distribution about the largest observed mean of the distributions. The distribution of reduced shear has the largest IQR and standard deviation. The shape of the dissipation distributions for daytime light wind conditions remains similar in depth (similar IQR, skewness, kurtosis), but the mean and median decrease. The mean and median of reduced shear also decrease with depth indicating more stable conditions deeper in the ocean. Even under the daytime, light wind conditions, PDFs reveal instances of dynamically unstable conditions, which may be due to the overturning of the self-regulating diurnal thermocline. Overall, the distribution of TKE dissipation and reduced shear during the daytime light wind speed conditions are different than night time light wind or windy conditions, especially so in the upper 10 meters.

\subsubsection{Energy of the mean current}

The shear production term of the TKE equation is a sink term in the mean kinetic energy (MKE) equation. Thus, with an observed estimate of TKE dissipation and an assumption that the shear production term must supply the TKE that is ultimately dissipated, the MKE can provide insight into what are and are not reasonable sources for the energy that is ultimate dissipated. To provide an upper bound on TKE dissipation, it can be assumed that all of the near-surface MKE is converted to TKE through shear production and then dissipated over some time-scale.

Using the kinetic energy (KE) of the observed current (Fig. 2-14), the integrated kinetic energy is calculated over the upper 10 meters as

$$
\langle\mathrm{KE}\rangle=\frac{1}{2} \rho_{0} \int_{-10 \mathrm{~m}}^{0 \mathrm{~m}}|\vec{u}|^{2} d z
$$

where $|\vec{u}|$ is the magnitude of the current, and the KE has units of $\mathrm{J} / \mathrm{m}^{2}$. The observed current at $50 \mathrm{~m}$ was subtracted out to remove the geostrophic component of the current. Lacking observations of velocity above the shallowest current meter at $3.5 \mathrm{~m}$, we consider two scenarios to extrapolate the velocity to the surface: a slab layer or constant velocity (black lines in Fig. 2-14a-d), and linear shear from 3.5m to the surface with a surface current 
TKE Dissipation PDF
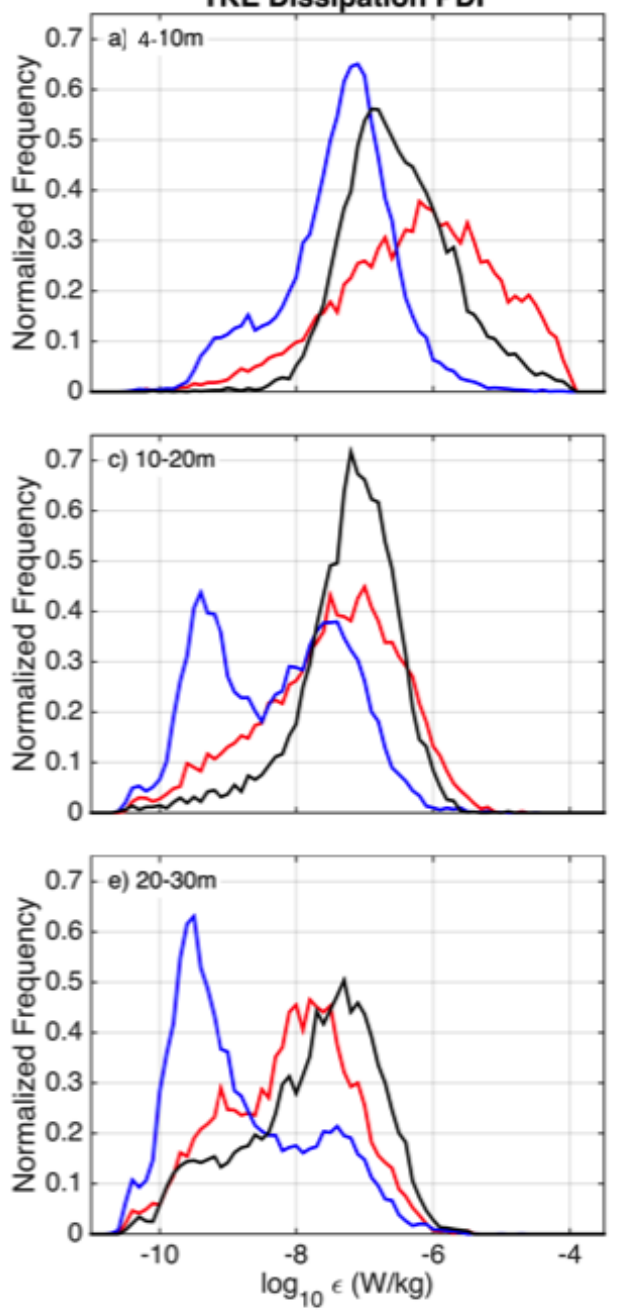

Reduced Shear PDF
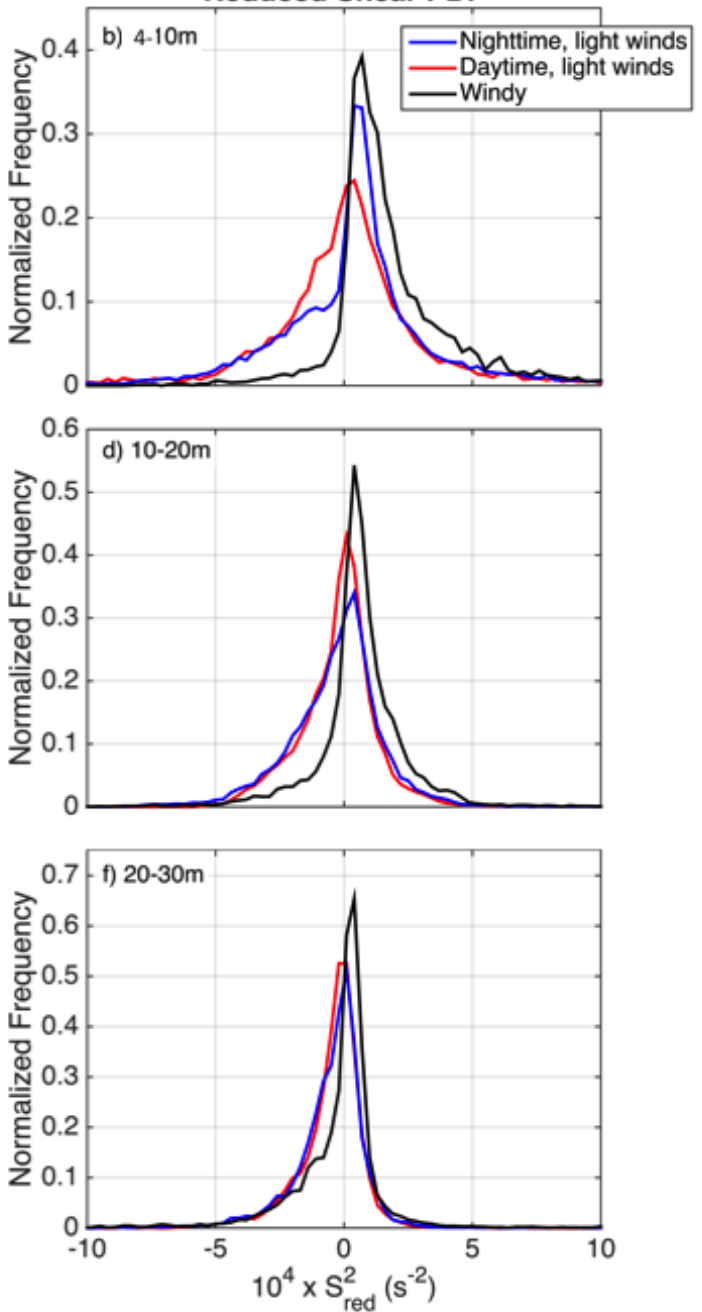

Figure 2-13: Empirical probability distribution functions of TKE dissipation and reduced shear, respectively, for depth bins of (a,b) 4-10 m (the shallowest observations limited the top of the near-surface bin), (c,d) 10-20 m, and (e,f) 20-30 m for nighttime light wind conditions (blue, $Q_{n e t}<0$, wspd $<5 \mathrm{~m} / \mathrm{s}$ ), daytime light wind conditions (red, Qnet $>0$, wspd $<5$ $\mathrm{m} / \mathrm{s}$ ), and windy conditions (black, wspd $>5 \mathrm{~m} / \mathrm{s}$ ). Each histogram is normalized such that the area under the curve is unity. The statistics of the distributions are presented in Tables 2.2 and 2.3 . 
twice as fast as 3.5 meters (red line in Fig. 2-14a-d). An integrated TKE dissipation based on glider observations is also calculated as

$$
\langle\epsilon\rangle=\rho_{0} \int_{-10 \mathrm{~m}}^{0 \mathrm{~m}} \epsilon d z
$$

and multiplied by a reference density to provide units of $\mathrm{W} / \mathrm{m}^{2}$. These quantities are shown in Fig. 2-14a and b. The ratio between integrated $\langle\mathrm{KE}\rangle$ and $\langle\epsilon\rangle$ provides a dissipation time-scale $\left(\mathrm{t}_{\epsilon}\right)$,

$$
\mathrm{t}_{\epsilon}=\frac{\langle\mathrm{KE}\rangle}{\langle\epsilon\rangle}
$$

which can be physically interpreted as the required time-scale for dissipation of the entire mean current of the upper $10 \mathrm{~m}$ in order to explain the magnitude of the observed TKE dissipation (Fig. 2-14c,d). This estimate assumes a local dissipation of mean shear. For the mean shear to provide enough energy to explain the observed TKE dissipation during the daytime under conditions of enhanced dissipation (high-dissipation, low-wind days), the entire current would have to be continuously converted to TKE and then dissipated in $10^{2}$ $10^{3}$ seconds or about 2-15 minutes. When assuming a surface current twice as fast as at $3 \mathrm{~m}$, the dissipation time-scale changes only by a factor of 2 or 3 . This is an improbably fast time-scale to reform and then dissipate the entire mean current.

Wind work is also a source of mean kinetic energy. The contribution of wind work to kinetic energy energy over a depth $H$ can be written generally as:

$$
\frac{\partial\langle\mathrm{KE}\rangle}{\partial t}=\int_{-H}^{0 \mathrm{~m}} u(z) \cdot \frac{\partial \tau}{\partial z} d z=[u(0) \cdot \tau(0)]-[u(-H) \cdot \tau(-H)]-\int_{-H}^{0 \mathrm{~m}} \tau(z) \cdot \frac{\partial u}{\partial z} d z
$$

where $\tau$ is the wind stress. If you consider $H$ as the depth at which $u$ and $\tau$ go to zero, and assume that the current and wind stress are aligned and that the current increases closer to the surface, then

$$
\frac{\partial\langle\mathrm{KE}\rangle}{\partial t}<u(0) \tau(0)
$$

and $\tau(0)$ can be estimated from the mooring. This is the most generous estimate of wind work. If all of the mean kinetic energy is converted to TKE through shear production and then dissipated, then $\langle\epsilon\rangle \sim u(0) \tau(0)$. Given the observed TKE dissipation and wind stress, a minimum necessary surface current can be estimated for wind work to explain the magnitude of the observed enhanced dissipation (here for the upper 10 meters). 


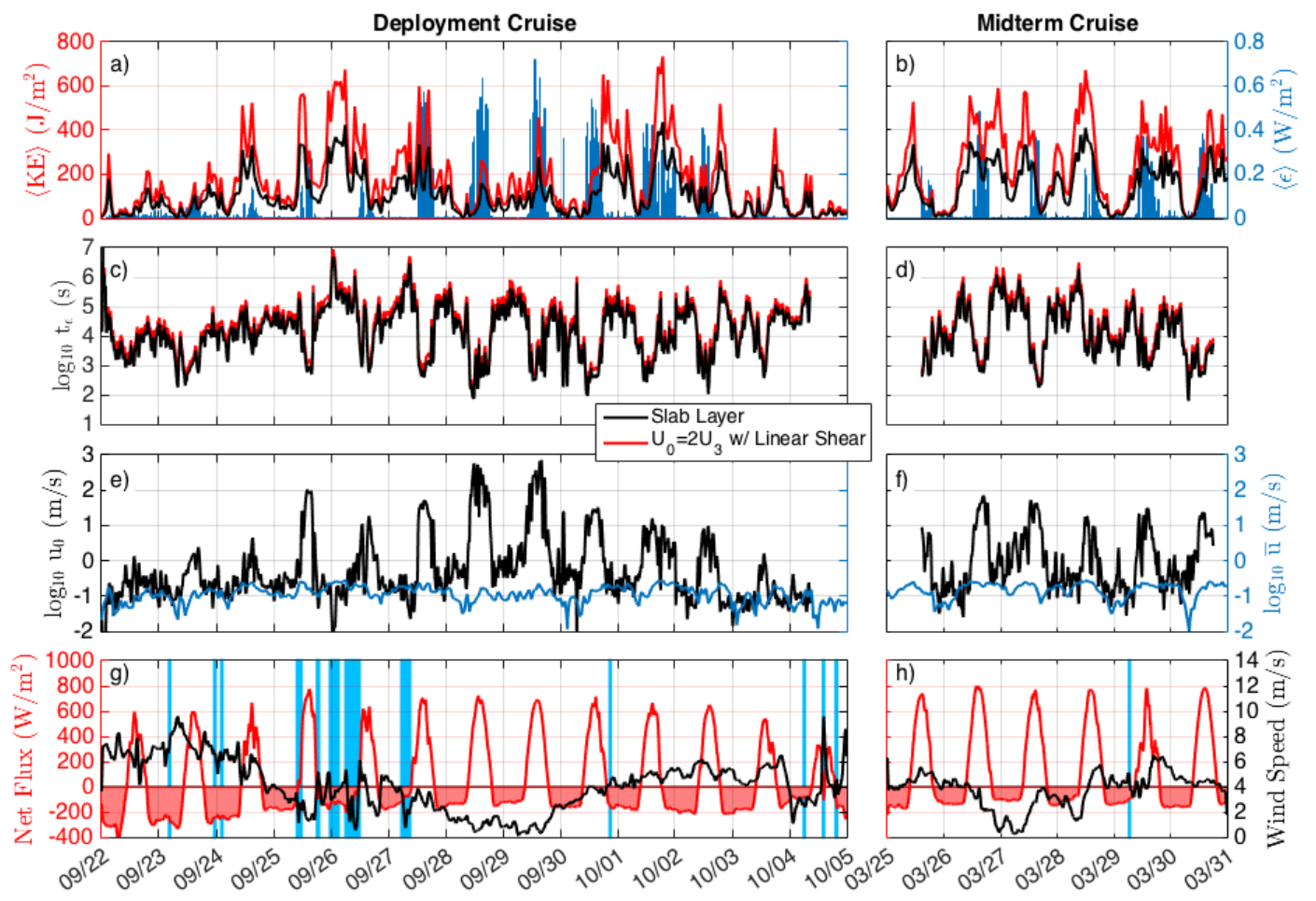

Figure 2-14: The upper 10 meter (a,b) integrated kinetic energy from the ADCP (Eq. 2.8, black line assumes slab layer above 3.5 meters and red line assumes a linear shear with a surface current twice as fast as 3.5 meters) and integrated TKE dissipation from the gliders (blue bars, Eq. 2.9) and (c,d) dissipation time-scale (Eq. 2.10). (e,f) The required speed of a slab layer for wind work to explain the observed TKE dissipation of the upper 10 meters (black line, Eq. 2.12) and mean current of the upper 10 meters (blue line). The meteorological conditions at the SPURS mooring $(\mathrm{g}, \mathrm{h})$ with the net surface heat flux in red, wind speed in black, and times of active precipitation in light blue during the deployment (left-side, 9/15/2012 - 10/5/2012) and midterm (right-side, 3/25/2013 - 4/6/2013) cruises. Note the sharp decrease in the dissipation time-scale in (c) and (d) and unrealistic surface current required in (e) and (f) during daytime conditions of diurnal warming and observed enhanced dissipation. 
This estimate is shown in Fig. 2-14e and $\mathrm{f}$ along with the observed current. Under daytime conditions with observed enhanced dissipation, the surface current that would be required to balance Eq. 2.12 ranges from 10 to $100 \mathrm{~m} / \mathrm{s}$ to explain the magnitude of the dissipation. Outside of the periods of enhanced daytime dissipation, the surface current necessary for wind work to explain the integrated TKE dissipation of the upper $10 \mathrm{~m}$ is the same order of magnitude as the observed current.

Both calculations are an overestimate of available energy that can be converted to TKE dissipation as not all MKE is converted to TKE, let alone TKE dissipation, and it has been assumed that the mean current and wind stress are aligned at all times. The unrealistic estimates of a dissipation time-scale and surface current provide evidence that a surface-forced shear mechanism is unlikely the sole cause of the observed enhanced daytime dissipation.

\subsection{Summary and Discussion}

\subsubsection{Summary}

The major results can be summarized as follows:

1. The observed enhanced TKE dissipation during the daytime is associated with a nearsurface stable layer due to diurnal warming. The diurnal cycle of dissipation is oppositely phased from previous observations with the highest values occurring during the daytime rather than at night (e.g. Soloviev and Vershinsky, 1982; Soloviev et al., 1988; Moum et al., 1989; Brainerd and Gregg, 1993a,b). The largest values of TKE dissipation occur earlier in time than peaks in enhanced shear and stratification associated with the diurnal warm layer. This signal disagrees with the canonical view of near-surface ocean turbulence.

2. Two different one-dimensional models, the Kantha-Clayson model (a 2nd-moment turbulence closure model) and the Price-Weller-Pinkel (a dynamical instability model), do not reproduce the enhanced daytime dissipation. However, the models appropriately predict the dissipation associated with wind-driven mixing events and nocturnal convection.

3. Monin-Obukhov Similarity Theory does not describe the enhanced daytime dissipation. It does however, properly describe the turbulence of the near-surface ocean 
under unstable forcing conditions. The disagreement between MOST prediction and the observations during the daytime warrants further investigation into the cause of the higher than MOST-predicted TKE dissipation observations.

4. Diurnal warm layer shear is present in the observations during the SPURS-I cruises. The shear layer is associated with strong stratification and is shown to be, on average, dynamically stable. The largest TKE dissipation is observed under the most gravitationally stable conditions. However, detailed observations of the current of the upper few meters of the ocean where the strong diurnal warming events occur are not available from the SRURS-I field campaign. Additionally, neither the kinetic energy of the current in the near-surface ocean nor energy input from the wind appears large enough to supply the energy that is being dissipated.

\subsubsection{Discussion}

Surface forcing alone does not appear to explain the observed enhanced TKE dissipation in the diurnal warm layer, and the DWL shear does not appear to be the sole cause of the high dissipation. However, the DWL shear may be a necessary component of the enhanced dissipation as both are present when the net surface heat flux is into the ocean. The influences of non-breaking surface gravity waves and internal waves propagating along and interacting with the diurnal thermocline need to be further investigated as possible sources of the observed enhanced TKE dissipation. A discussion of internal waves is provided in Chapter 3. The small scale of stable boundary layers in the ocean make proper observations challenging with very few direct observations of the very near-surface (such as Kudryavtsev and Soloviev, 1990). The results of this study demonstrate the need for higher resolution observations of temperature, salinity, and currents in the near-surface ocean, and present an interesting observational, theoretical, and modeling problem. 


\section{Chapter 3}

\section{Near-surface horizontal velocities induced by deep internal waves}

\section{$3.1 \quad$ Introduction}

Internal waves (IWs) are a ubiquitous feature of the stably stratified ocean (Garrett and Munk, 1979). Within the near-surface ocean, the traditional concept of a well-mixed layer precludes vertical propagation of internal waves with high vertical wavenumbers. However, low frequency waves, such as the inertial or dominant tidal frequency, can be observed throughout the water column. In addition, the manifestation of deep internal waves is still possible in the boundary layer through evanescent (or decaying) modes. D'Asaro (1978), hereafter D78, proposed a mechanism for relatively strong horizontal velocities in the mixed layer caused by IWs with large decay scales from deep in the ocean.

The classical model of the upper ocean includes an active convectively mixed layer during the nighttime when the net heat flux cools the near-surface ocean, creating a positive density anomaly at the surface and thus Rayleigh-Taylor instability (Moum et al., 1989; Brainerd and Gregg, 1993a,b). When the near-surface ocean stratifies during the daytime due to penetrative solar radiation creating a diurnal warm layer, a stable boundary layer forms, and conditions become favorable for a greater range of propagating IWs (IWs generally can exists between $f$, the Coriolis parameter, and $N$, the local buoyancy frequency of the ocean). During periods of relatively light winds and high solar insolation, the upper few meters of the ocean can warm and then cool several degrees over the course of a diurnal cycle (Price 
et al., 1986). Momentum trapped by the stable stratification of the diurnal warm layer generates shear across the layer (Chapter 2). In this stratified and sheared environment, IWs are able to transport momentum at speeds different than the mean flow, and have complicated propagation characteristics (Garrett and Munk, 1979; Sutherland, 2010).

Under these daytime diurnal warming conditions Clayson et al. (2016b) found greater than expected Turbulent Kinetic Energy (TKE) dissipation $\left(\epsilon>1 \times 10^{-5} \mathrm{~W} / \mathrm{kg}\right)$ in the upper 30 meters of the ocean during the Salinity Processes in the Upper ocean Regional Study, or SPURS-I, field campaign. The observed values were greater during the daytime than nighttime. In Chapter 2, we concluded that it is unlikely that surface forcing could be the sole cause of the observed enhanced TKE dissipation near the surface. This conclusion is consistent with the findings of Sutherland et al. (2014), which note that TKE dissipation rates during the daytime are greater than expected and inconsistent with expectations from surface forcing. Specifically, they note that traditional Thorpe scaling does not scale with the boundary layer depth during the daytime; it does during the night under convection. Thorpe scaling is an estimate of the mixing length based on the statistics of the size of turbulent overturns in the ocean boundary layer.

Although the diurnal warm layer (DWL) has been studied for several decades (e.g. Stommel and Woodcock, 1951; Woods, 1968; Houghton, 1969), the relatively small depth of these layers makes them difficult to observe. The atmospheric stable boundary layer (the analogous layer in the atmosphere occurs during the nighttime) is orders of magnitude greater in vertical scale than the oceanic one, and thus easier to observe. Much of the knowledge concerning stable boundary layers comes from the atmosphere (e.g. Wyngaard et al., 1971; Pahlow et al., 2001; Poulos et al., 2002; Cheng et al., 2005; Sun et al., 2012). The daily cycle of the stability of the atmospheric and oceanic boundary layers are opposite. During the daytime atmospheric convective boundary layer, the ocean can experience a stable boundary layer if wind speeds are low enough for buoyant turbulent suppression by penetrative solar radiation to overwhelm mechanically driven wind mixing (Soloviev and Vershinsky, 1982). Additionally, the influence of IWs on and other complex features of the stable boundary layer is still an important topic of research in the atmospheric community (Mahrt, 2014), and will likely be similarly important for the ocean. Here, we follow on with a focus on internal gravity waves.

IWs were observed during daytime, low-wind conditions during the SPURS-I field cam- 
paign (Hodges and Fratantoni, 2014). The dominant observed wave with the DWL was estimated to have a period (moving with the mean flow) of 6 hours and $1300 \mathrm{~m}$ wavelength. The observed wave is relatively high frequency $(\omega \gg f)$. IWs in the upper ocean have been a topic of research for over four decades and many of the fundamental questions raised by Gregg and Briscoe (1979) have yet to be answered. The 1970s saw significant advances in observations and theory of IWs. IWs in the near-surface ocean are less studied, but nearsurface IW signals have been observed in sea surface temperature by Walsh et al. (1998) and Farrar et al. (2007), in addition to those observed by Hodges and Fratantoni (2014). Pinkel (1975) observed IWs in the upper ocean using the floating platform "FLIP."

On average, IWs can be considered as a universal spectrum such as that of Garrett and Munk $(1975,1972,1979)$. The idea of a universal spectrum in the upper ocean is less clear. Roth et al. (1981) show that a universal spectrum breaks down nearer the surface of the ocean. This is likely due to added complexities in the surface layer. For example, Sethuraman (1980) showed that persistent breaking atmospheric IWs in the stable atmospheric boundary layer over the ocean were found to significantly increase the turbulence and the TKE dissipation by almost two orders of magnitude. Concepts from the stable atmospheric boundary layer are likely applicable to the ocean. We hypothesize that the deep ocean can serve as a source of energy for near-surface mixing, and that the details of the upper ocean stratification are important in modulating the internal waves that reach the surface and affect the surface layer.

IWs can generate large values of TKE dissipation through non-linear breaking mechanisms. Observations of larger scale wave breaking (Moum et al., 2007) have shown values of TKE dissipation similar to those of Clayson et al. (2016b). Novel observational platforms such as the Air-Sea Interaction Profiler (ASIP, Ward et al., 2014), and Rockland Scientific MicroRider (e.g. Fer et al., 2014) have allowed for the exploration of turbulence near the surface of the ocean that could not be previously explored due to the nature of traditional microstructure profiling instrumentation (Callaghan et al., 2014). The novel platforms are the basis of the new observations in this study.

IWs are a possible mechanism of TKE shear production and ultimately dissipation during the stratified daytime upper ocean. We present evidence of the collocation of strong stratification and large TKE dissipation in the upper ocean. An important measure of the 
stably stratified ocean is the buoyancy frequency, $N$, where

$$
N^{2}=\frac{g}{\rho_{0}} \frac{\partial \bar{\rho}}{\partial z}
$$

and $g$ is the gravitational constant, $\rho_{0}$ is a reference density, and $\rho$ is the measured density. The focus of this study is on how the stably stratified near-surface diurnal warm layer creates favorable conditions for strong shear due to IWs, ultimately causing turbulence and the observed enhanced TKE dissipation.

Section 2 contains details on the observational platforms and cruises, and derived variables such as isopycnal displacement. A discussion of the observed stratification, TKE dissipation, and IWs from the SPURS-I field campaign is provided in Section 3. The D78 mechanism is derived and used to explain vertically coherent horizontal velocities observed in the mixed layer in Section 4. Section 5 extends the D78 mechanism to include a diurnal thermocline, and provides a discussion of implications for IWs on the diurnal thermocline and horizontal kinetic energy in the diurnal warm layer. A summary and conclusions are provided in Section 6.

\subsection{Data}

Three research cruises and year-long instrument deployments were associated with the SPURS-I field campaign. A "deployment" cruise occurred in the fall of 2012, a "mid-term" cruise in spring 2013, and "recovery" cruise in fall 2013. This analysis focuses on a year-long "central mooring" record centered at $24.5^{\circ} \mathrm{N}, 38^{\circ} \mathrm{W}$ (Farrar et al., 2015), as well as Slocum gliders and Wave Gliders.

\subsubsection{SPURS-I Central Mooring}

The central mooring had surface and subsurface components providing high temporal- and vertical-resolution observations. The surface component includes an IMET system (Hosom et al., 1995; Colbo and Weller, 2009), providing one-minute meteorological data such as wind speed and direction, shortwave and longwave radiation, precipitation, and sea surface temperature and salinity. A net surface heat flux is calculated similar to Chapter 2 as

$$
Q_{N}=Q_{L}+Q_{B}+Q_{P}+Q_{L W}+Q_{S W}
$$


where $Q_{N}$ is the net surface flux, $Q_{L}$ is the latent heat flux, $Q_{B}$ is the sensible heat flux, $Q_{P}$ is the heat flux due to the temperature difference between the sea surface and precipitation, $Q_{L W}$ is the outgoing longwave radiation, and $Q_{S W}$ is the incoming shortwave radiation. A positive flux is into the ocean. The turbulent fluxes are estimated using the COARE 3.0 algorithm (Fairall et al., 2003).

The subsurface component includes 31 fixed-depths CTDs in the upper 200 meters. Four upward-facing ADCPs (at 18, 75, 145, 295 meters depth), a point current meter at 3.0 meters, and Vector-Measuring Current Meters (VMCMs) at 502, 1005, and 1708 meters provide current measurements throughout the upper ocean. These are the same data used in Chapters 2 and 4 and Farrar et al. (2015).

\subsubsection{Slocum and Wave Gliders}

In addition to the central mooring and to obtain better spatial coverage of the SPURS-I study area, Liquid Robotics Wave Gliders (Daniel et al., 2011) were deployed during the field campaign, and remained in the water for a year-long mission (other than a few days during the midterm cruise for servicing). Three Wave Gliders moved about a $1^{\circ} \mathrm{x} 1^{\circ}$ box near the central mooring in repeating paths to provide good temporal and spatial coverage. The Wave Gliders have a surface component roughly the size of a surfboard and a subsurface component with wings that propel the board forward. The two components are connected by a 6 meter cable. The surface float is equipped with meteorological instrumentation, solar panels, and a CTD on the bottom side, nominally at $0.3 \mathrm{~m}$. The subsurface component is at $6 \mathrm{~m}$, and was also instrumented with a CTD.

Two Webb-Teldyne Slocum gliders were used during the deployment and midterm cruise. A total of 3,692 profiles around the mooring were conducted over 3 days of the two cruises. The gliders were equipped with Rockland Scientific MicroRiders to measure microstructure. The microstructure data are the focus of Clayson et al. (2016b) and Chapter 2. The gliders were ballasted light in order to better observe the near-surface ocean, and kept close to the mooring. Peterson and Fer (2014) and Fer et al. (2014) found the data from MicroRiders to be high quality and a useful platform for turbulence measurements. 


\subsubsection{Isopycnal Displacement}

The current meter observations in the upper ocean are taken hourly. To examine the ocean at higher time resolution, an isopycnal displacement is calculated from the temperature and salinity observations of the central mooring, which are five-minute observations. The isopycnal displacement is linearly interpolated from calculated potential density for each fixed-depth CTD. This is estimated for isopycnals every $0.01 \mathrm{~kg} / \mathrm{m}^{3}$. The depth is estimated from the stratified interior upward towards the surface, so that during nocturnal convection when an isopycnal may occur twice vertically, the deeper isopycnal is used.

\subsection{Observations of Stratification and TKE dissipation}

Longwave radiative and turbulent fluxes generally act to cool the ocean surface here(Brainerd and Gregg, 1993a; Clayson et al., 2016a), so without the stabilizing force of shortwave radiation, as occurs during the nighttime, the surface cools and convection ensues (RayleighTaylor instability). The canonical model of the upper ocean includes a convectively turbulent nighttime, and a quiescent daytime due to the stabilizing forcing of penetrative solar radiation (Webster et al., 1996; Soloviev and Lukas, 1997). However, Chapter 2 shows that the largest observed near-surface TKE dissipation values occur when the upper ocean is stratified during the daytime, which is further demonstrated by Fig. 3-1.

When the near-surface is stratified (Fig. 3-1e,f) due to heating by the shortwave radiation, relatively large values of TKE dissipation are observed (Fig. 3-1g,h). The largest values are confined to the upper $10 \mathrm{~m}$, but the enhanced dissipation signal can be observed to $30 \mathrm{~m}$ during the peak stratification. High stratification is coincident with the enhanced TKE dissipation.

Breaking the upper ocean into more discrete regions, it becomes apparent that the enhanced TKE dissipation is associated with an increase in near-surface stratification (upper $5 \mathrm{~m}$, Fig. 3-2c,d). Although enhanced TKE dissipation occurs deeper than the upper 5 meters of the ocean, examining 10-15 m and 20-25m bins (Fig. 3-2e,f,g,h) the association with stratification is less clear. This indicates that the phenomenon responsible for the enhanced dissipation requires near-surface stratification, but since high dissipation is observed deeper in the ocean, the phenomenon can also cause turbulence away from the stratification. The relationship is considered further by examining the upper $10 \mathrm{~m}$ averaged over the daylight 

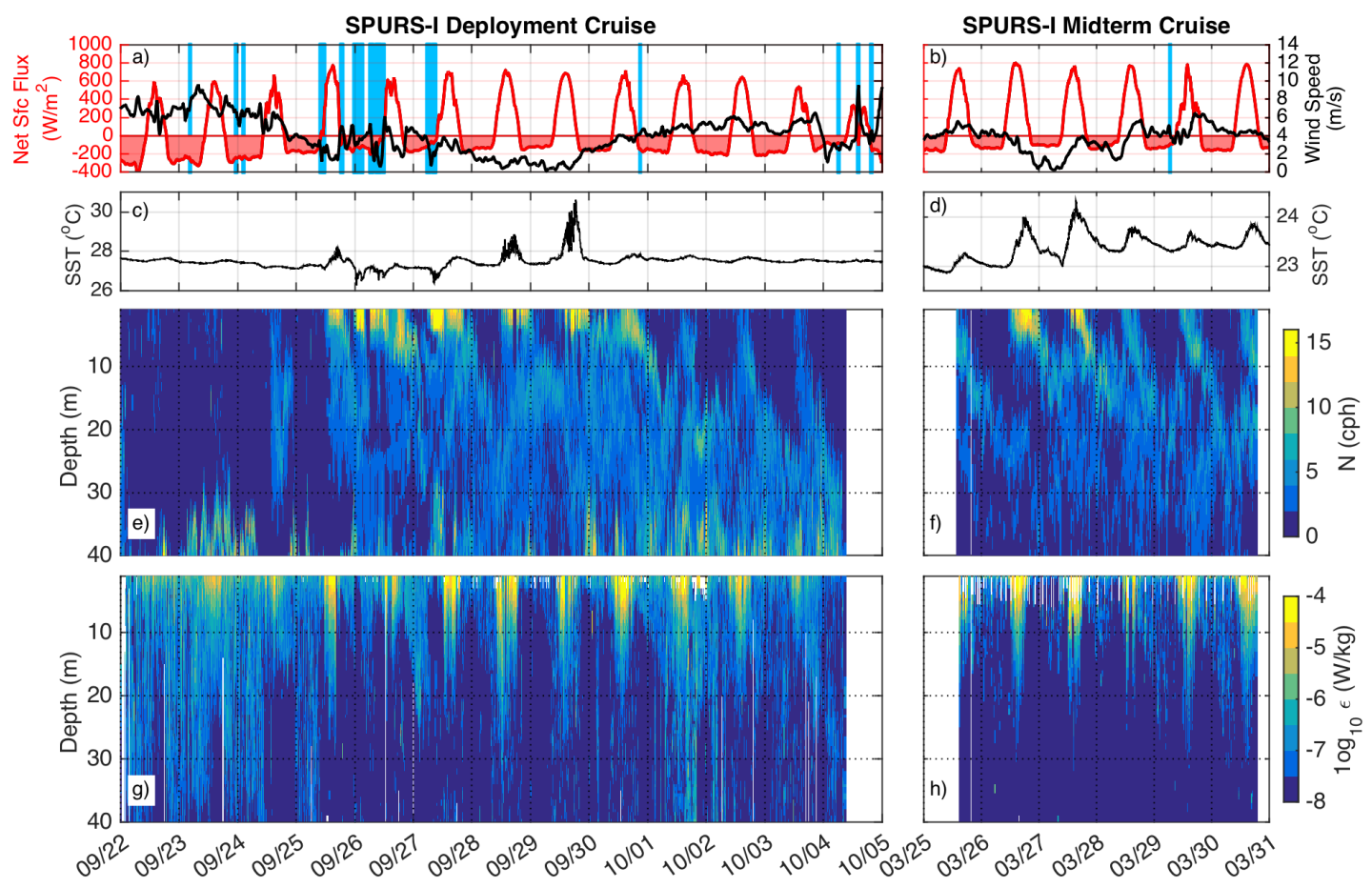

Figure 3-1: The meteorological conditions at the SPURS mooring (a,b) with the net surface heat flux in red, wind speed in black, and times of active precipitation in light blue. Sea surface temperature (c,d), buoyancy frequency in cycles per hour (e,f), and turbulent kinetic energy dissipation $(\mathrm{g}, \mathrm{h})$ from the Slocum gliders during the deployment (left-side, 9/15/2012 - 10/5/2012) and midterm (right-side, 3/25/2013 - 4/6/2013) cruises. 

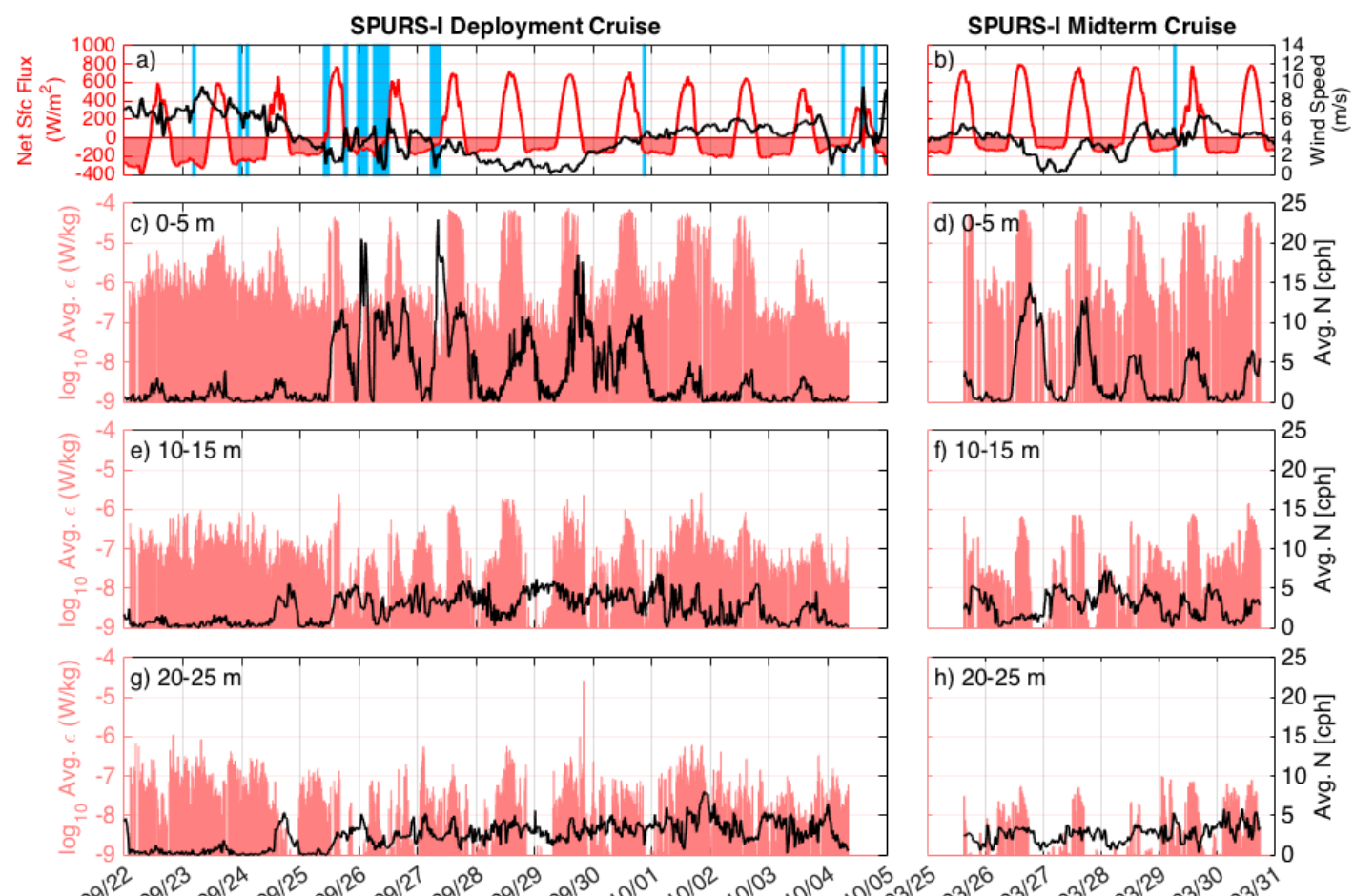

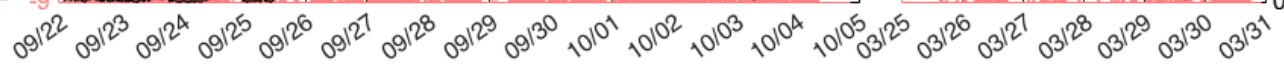

Figure 3-2: The meteorological conditions at the SPURS mooring $(a, b)$ with the net surface heat flux in red, wind speed in black, and times of active precipitation in light blue. Depth averaged buoyancy frequency (black line) and turbulent kinetic energy dissipation (red bars) for 0-5 meter bin (c,d), 10-15 meter bin (e,f) and 20-25 meter bin $(\mathrm{g}, \mathrm{h})$ from the gliders during the deployment (left-side, 9/15/2012 - 10/5/2012) and midterm (right-side, 3/25/2013 $4 / 6 / 2013)$ cruises. 


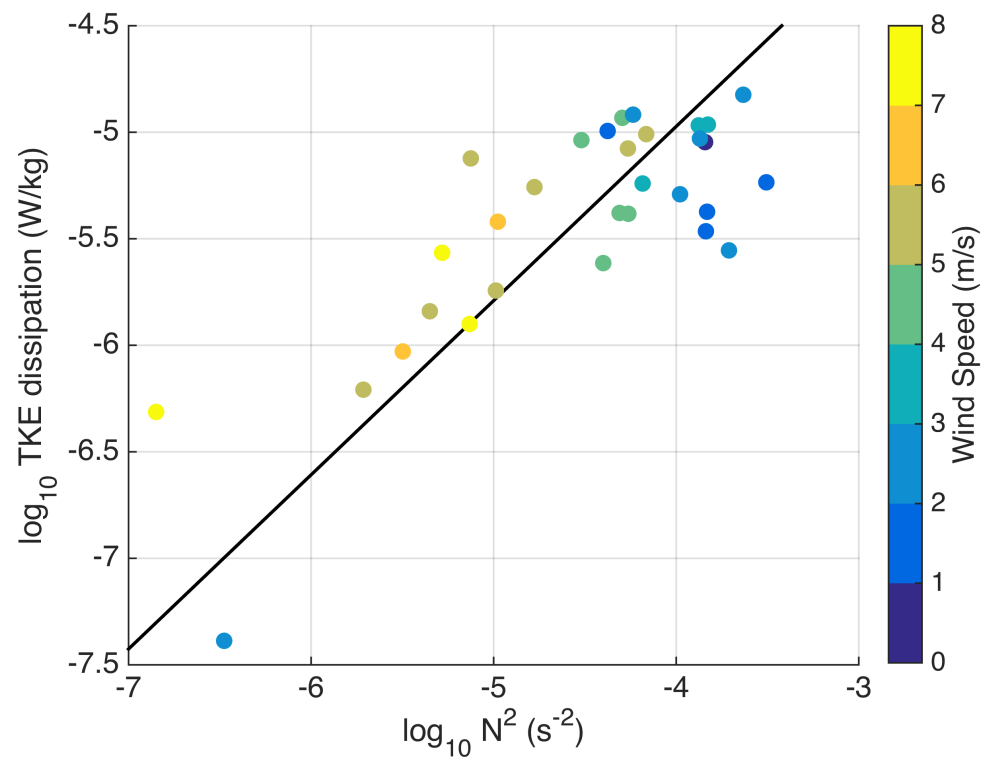

Figure 3-3: Turbulent kinetic energy dissipation versus buoyancy frequency squared, averaged over the daylight hours of a day over the upper 10 meters from the Slocum gliders during SPURS-I deployment (9/15/2012 - 10/5/2012) and midterm (3/25/2013 - 4/6/2013) cruises. The color represents the windspeed from the mooring averaged over the same time period. The black line is the least squares best fit line.

hours, as is done in Fig. 3-3. There is a clear relationship between the logarithm of mean TKE dissipation and stratification during the daylight hours. As stratification increases $\left(N^{2}\right)$ so does the mean dissipation of the upper $10 \mathrm{~m}$. The correlation between the logarithms is statistically significantly (by $\mathrm{r}^{2}$ value, $\mathrm{r}^{2}=0.82$ ). Since wind-driven mechanical mixing will act to destroy the near-surface stratification, the largest stratification occurs on the calmest days. Shay and Gregg (1986) likewise note intermittent high TKE dissipation events associated with a stable upper ocean that they associate with shear instability.

The observational evidence suggests that the cause of the enhanced dissipation is not solely surface induced (as has been shown in Chapter 2) and is related to the existence of stratification and phenomena associated with it such as IWs. Although IWs are generally considered within stably stratified regions of the ocean, they can also cause shear in unstratified layers through evanescent or decaying modes (D'Asaro, 1978; Sutherland and Yewchuk, 2004; Sutherland, 2006).

Coherent variability in surface temperature and salinity, interpreted to be IWs (later quantitatively shown), is observed during the daytime under conditions with diurnal strati- 


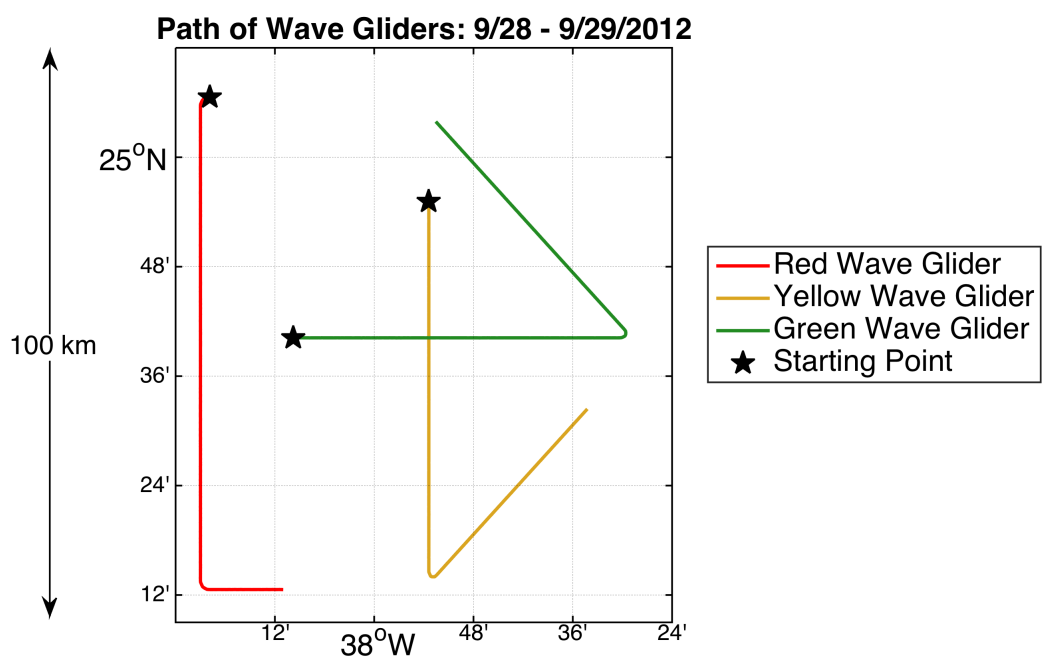

Figure 3-4: Path of the three Wave Gliders deployed during the SPURS-I field campaign for the time period of September 28 - 29, 2012.

fication by the Wave Gliders (Fig. 3-5). The Wave Gliders are a moving platform (Fig. 3-4) and do not move at a consistent speed. Here, Wave Glider observations are explored as a function of distance to more readily show horizontal gradients. The gradients of temperature (Fig. 3-5a,c,e) and salinity (Fig. 3-5b,d,f) are shown for the three Wave Gliders in the water during two large amplitude diurnal warming days on September 28 and 29, 2012.

Coincident variability in temperature and salinity occur while the upper ocean is stratified. Positive temperature gradients (warmer) correspond with observed positive salinity gradients (saltier). This indicates that warmer, saltier water is being advected downward and vice versa. The variability can be thought of as a time variability at a particular point in time or as space variability across a distance at a specific time. Here, we consider the latter. IWs advect the average vertical gradients up and down, and manifest as horizontal gradients. In the case of oscillatory advection, vertical gradients should be and are proportional to the observed horizontal gradients. This is consistent across all three Wave Gliders.

While IWs are a possible cause of the observed enhanced dissipation and do qualitatively fit the physical conditions observed, a more quantitative analysis provides further evidence that the coherent variability is IWs propagating along the DWL. A convenient method of examining temperature and salinity gradients concurrently is using a Turner angle ( $\mathrm{Tu})$ type diagram. This approach is traditionally used for vertical gradients (e.g. Schmitt, 1994). 

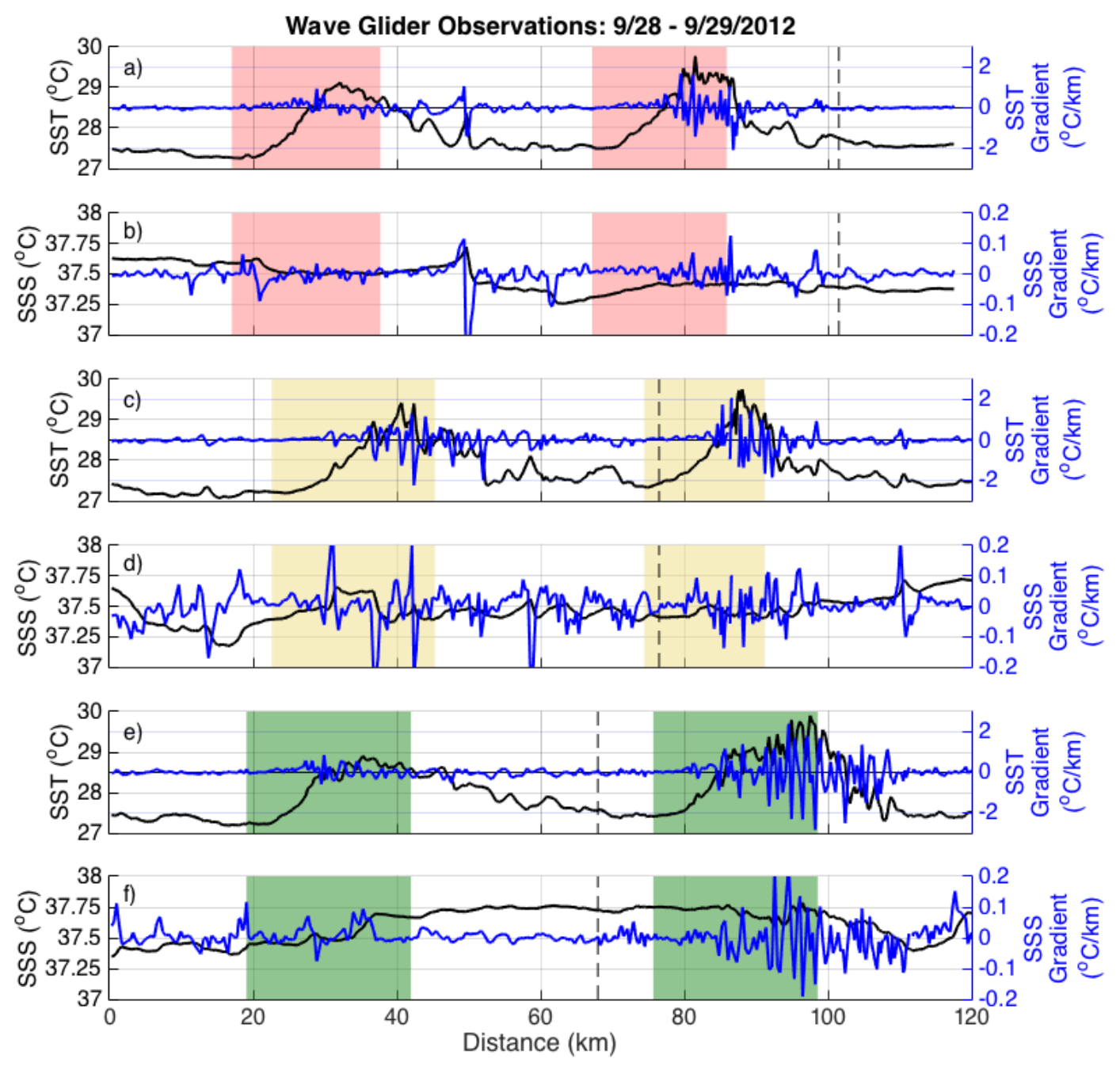

Figure 3-5: Observations from the three Wave Gliders of (a,c,e) temperature and (b,d,e) salinity (black lines) and corresponding gradients (blue lines) at a depth of $0.3 \mathrm{~m}$ for the time period of September 28 - 29, 2012. The shaded regions indicate when the the set surface heat flux is heating the ocean. The color choice identifies the Wave Glider, consistent with Fig. 3-4. The vertical dash line indicates turning points of the gliders. The x-axis is selected as distance to highlight the horizontal gradients observed by the gliders. 
Specifically, Tu is defined using a four-quadrant arctangent (atan2) defining the y-axis as a temperature gradient and the $\mathrm{x}$-axis as a salinity gradient (as done for T-S plots) normalized such that the units are comparable density gradients, defined as

$$
T u=\operatorname{atan} 2\left(\alpha T_{z}+\beta S_{z}, \alpha T_{z}-\beta S_{z}\right)
$$

where $\alpha$ is thermal expansion coefficient, $\beta$ is the saline contraction coefficient, $T_{z}$ is a vertical temperature gradient, and $S_{z}$ is a vertical salinity gradient. A similar angle can be used to examine horizontal gradients as well (e.g. Tippins and Tomczak, 2003). Defining the gradients in this way provides six physical regimes. Diurnal warming events would be expected in the stable, warmer, saltier regime, and rain events for this region would generally fall in the stable, cooler, fresher regime.

If IWs are present and the primary cause of variability, the Tu associated with the horizontal temperature gradients should have the same value as the Tu of the vertical gradients. This is because IWs can advect isopycnals up and down and convert vertical gradients into horizontal ones. The traditional regimes of Tu are shown in Fig. 3-6 and vertical gradients observed from two days of SPURS-I deployment cruise are considered in Fig. 3-7a. If the observed phenomenon is density compensated, the Tu of the gradients would fall along the $1: 1$ line. In order to maintain a constant density across a temperature gradient for example, there must be a coincident equal, but opposite change in density associated with a salinity gradient. This is why the Turner angle includes the thermal expansion and saline contraction coefficients.

Here, the the $\mathrm{Tu}$ of the horizontal gradients (Fig. 3-7b) fall along the same best fit lines as the vertical gradients (Fig. 3-7a, the dotted lines are the best fits for the diurnal warming and rainfall events observed during the deployment cruise). The positive and negative gradients in the horizontal T-S plot (Fig. 3-7b) demonstrate that advection of the mean gradient is occurring both upwards and downwards, as would be expected with internal waves. This occurs over a relatively short period of time, and internal waves are the only oscillatory phenomenon in the ocean that would produce this result. This is direct observational evidence of IWs present during the daytime under diurnal warming conditions in the upper few meters of the ocean.

All the cases of enhanced daytime TKE dissipation are associated with stabilizing surface 


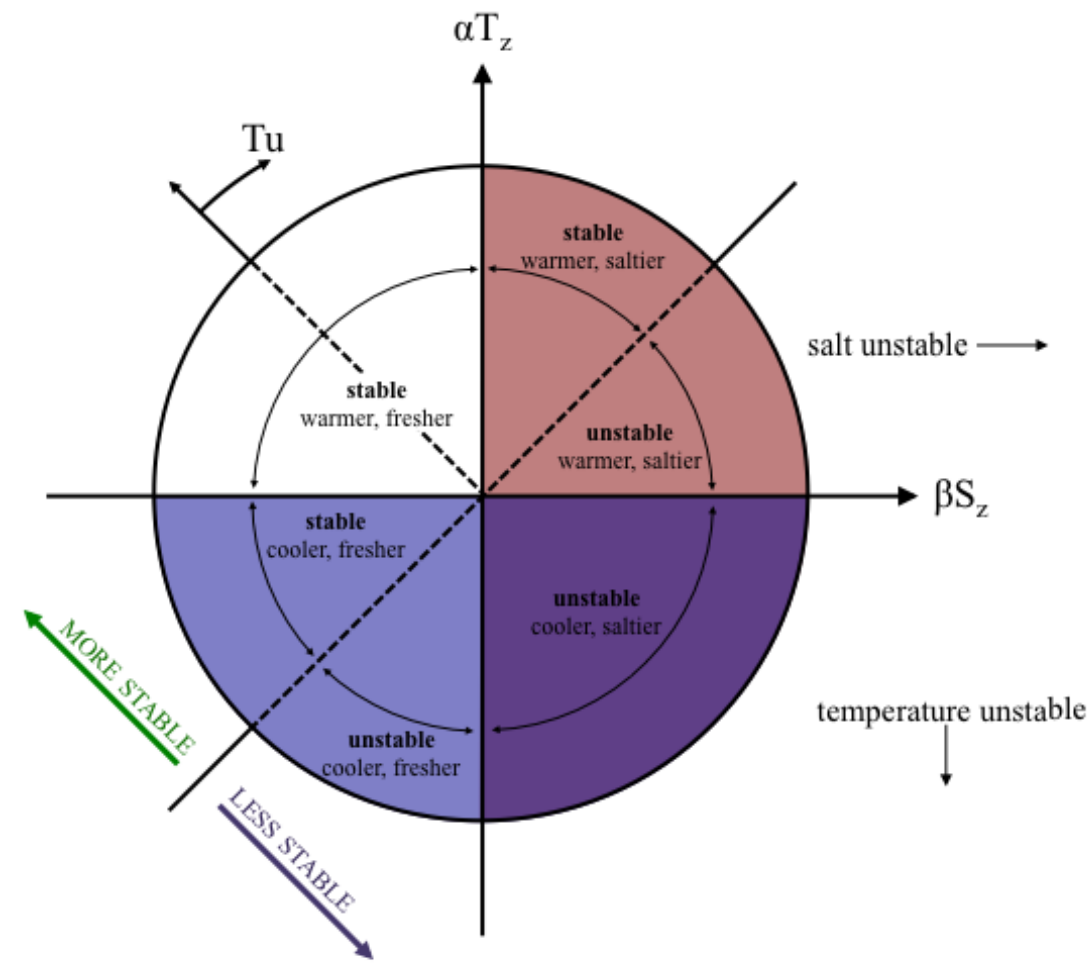

Figure 3-6: Turner angle diagram modified after Ruddick (1983) and Schmitt (2011). The vertical axis is the vertical density gradient due to temperature, and the horizontal axis is the vertical density gradient due to salinity. The Turner angle ( $\mathrm{Tu}$ ) is defined clockwise from where marked. "Stable or "unstable" references the static stability of the vertical density gradient, and the temperature and salinity identifiers denote the water mass atop. For example, "warmer, saltier" means that warmer and saltier water is atop relatively cooler and fresher water. 

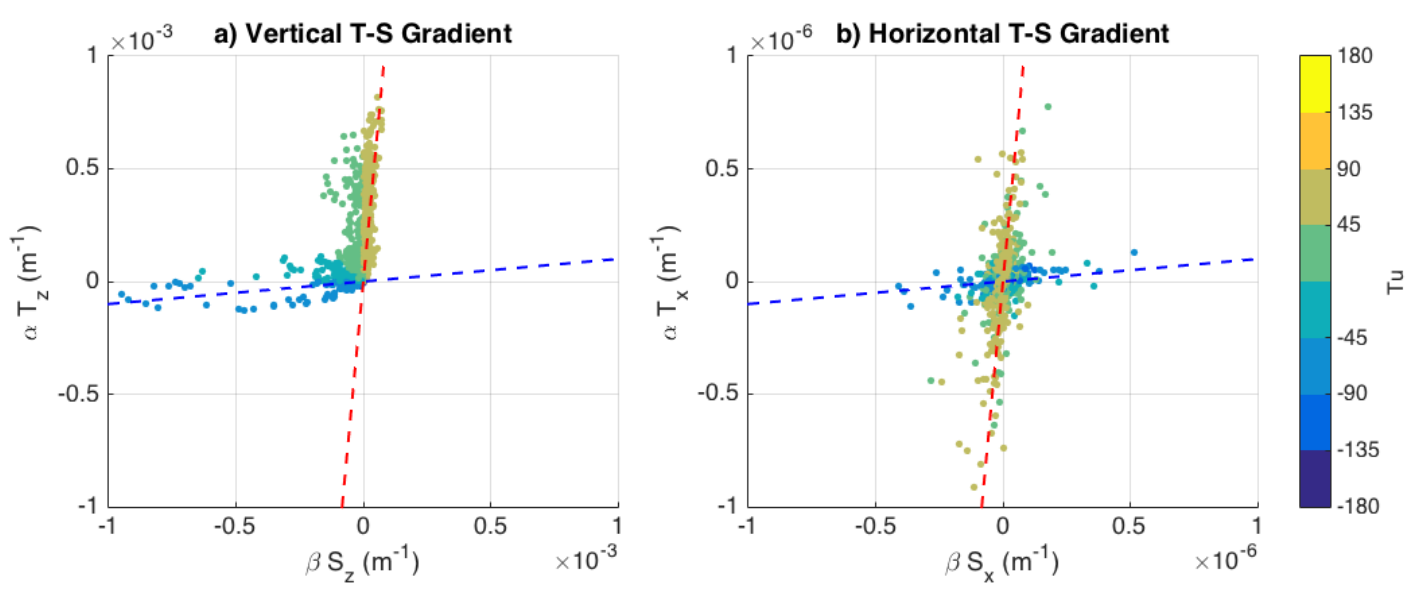

Figure 3-7: Scaled (a) vertical $(0.3$ to $6 \mathrm{~m})$ and (b) horizontal temperature gradients (from the $0.3 \mathrm{~m}$ CTD) from the Wave Gliders for the time period of September 28 - 29, 2012. The gradients are scaled by the thermal expansion coefficient and saline contraction coefficient, respectively, such that temperature and salinity are shown as comparable density changes. The color represents the Turner angle, and axis are those explained by Fig. 3-6. The blue and red dotted line represent the best fit for the freshwater lens and diurnal warming regimes for the days, respectively.

forcing from solar radiation and stratification near the surface. IWs are a possible turbulent shear production mechanism, as is required under stabilizing surface forcing conditions to explain the observed enhanced TKE dissipation (TKE dissipation must occur through a shear production mechanism when the surface buoyancy flux is stabilizing). The next step is to explore how IWs with a stratified near-surface ocean can cause turbulence. However, before focusing on the case with a stratified near-surface ocean, it is a worthwhile exercise to explore the traditional thinking of a mixed layer atop a stratified interior ocean, as was done in D78.

\subsection{Mixed Layer induced velocities}

Long deep IWs that have much larger decay scales than the mixed layer can induce horizontal velocities with same magnitude as turbulent velocities (those measured by microstructure, for example) induced by wind stress. This was shown by D78 in a brief note using a Garrett and Munk type spectrum for deep IWs in the "stratified interior." Although the concept is physically probable, there is little mention of the direct effect of evanescent waves in the 

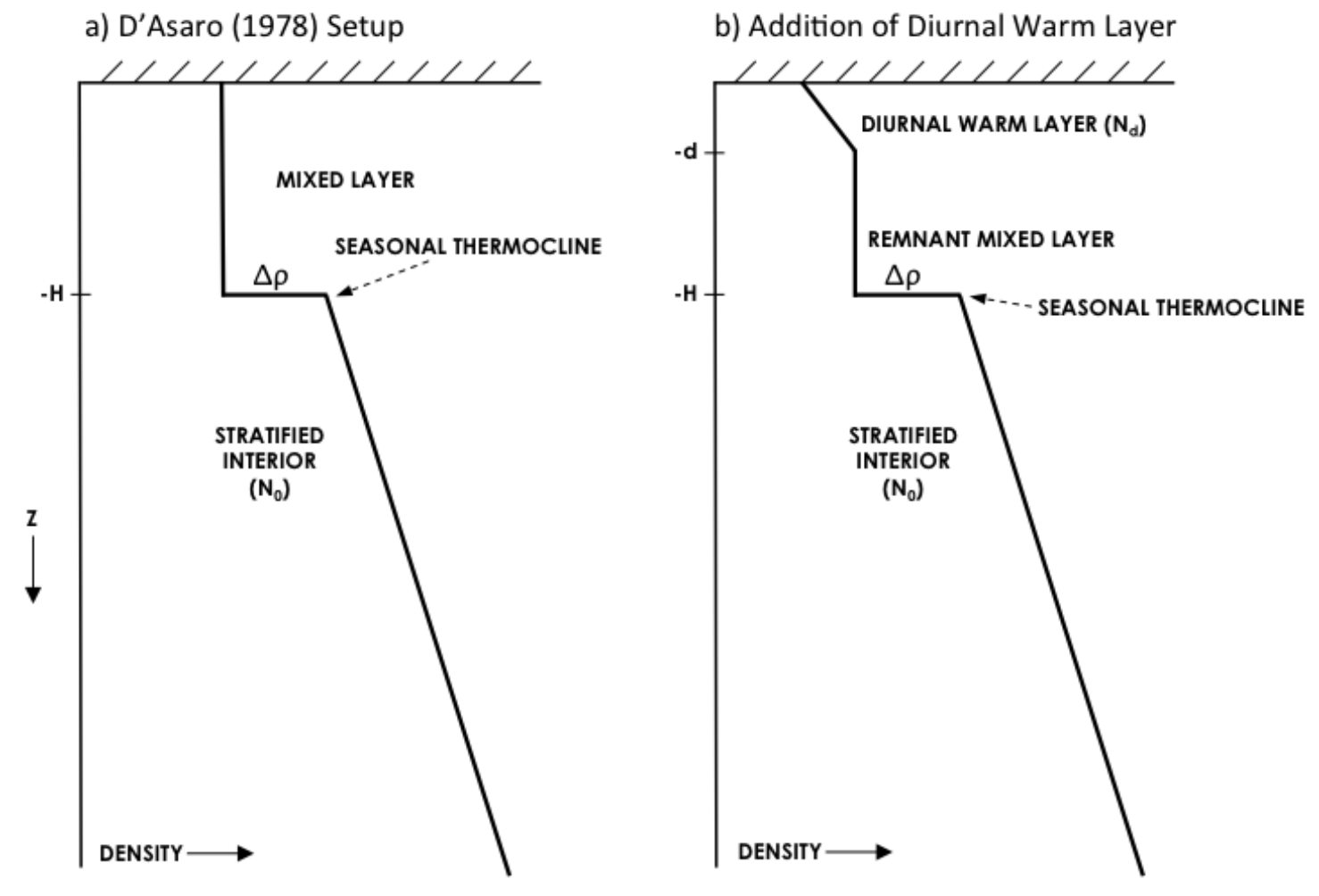

Figure 3-8: Simplified upper ocean density structure for (a) a well-mixed layer with seasonal thermocline treated as a density jump (This is Fig. 1 adapted from D'Asaro, 1978) and (b) a stratified diurnal warm layer above a so-called remnant mixed layer.

mixed layer in the scientific literature.

Starting with a simplified vertical profile of the ocean (Fig. 3-8a), the interior ocean is treated as a region of constant stratification, $N_{0}$, the seasonal thermocline is treated as a density jump of $\Delta \rho$, and the mixed layer is considered a unstratified slab layer $(N=0)$. In order to explore IWs in each region, the linearized Boussinesq momentum, thermodynamic, and continuity equations are combined into a linearized IW equation

$$
\frac{\partial^{2}}{\partial t^{2}}\left(\nabla_{H}^{2} w^{\prime}+\frac{\partial^{2} w^{\prime}}{\partial z^{2}}\right)+f^{2} \frac{\partial^{2} w^{\prime}}{\partial z^{2}}+N^{2} \nabla_{H}^{2} w^{\prime}=0
$$

where $w^{\prime}$ is the perturbation vertical velocity, $f$ is the Coriolis parameter, $N$ is the BruntVäisälä or buoyancy frequency, and $\nabla_{H}^{2}$ is the horizontal Laplacian (the details of the linearized IW problem can be found in: Gill, 1982; Lighthill, 1978; Kundu and Cohen, 
2008; Gerkema and Zimmerman, 2008; Sutherland, 2010). There is no mean flow in the problem, only perturbation velocities. A waveform is assumed for vertical velocity as $w^{\prime}(x, z, t)=W(z) e^{i(\alpha x-\omega t)}$. In this case, the wave propagation is assumed to be in the $\mathrm{x}$-direction, which can be done without any loss of generality. A flow will still be induced in the $\mathrm{y}$-direction. The $\mathrm{x}$-direction can be thought of as the direction of wave propagation. Thus, the linearized IW equation for a given density structure (consider layers with constant buoyancy frequency) is given by

$$
\frac{d^{2} W(z)}{d z^{2}}+\alpha^{2} \frac{N_{i}^{2}-\omega^{2}}{\omega^{2}-f^{2}} W(z)=0
$$

where $W(z)$ is the vertical structure of a wave within a density layer with a constant buoyancy frequency, for a given wavenumber $\alpha$ and frequency $\omega$. Each region will have a vertical wavenumber, $m^{2}$, defined as

$$
m^{2}=\alpha^{2} \frac{N_{i}^{2}-\omega^{2}}{\omega^{2}-f^{2}}
$$

where $N_{i}$ is the stratification of an $i$ th layer. For this case there are two layers: the mixed layer and stratified interior. The details of the derivation are in Appendix B. There are solutions for each region that can be matched using boundary conditions at the boundary between the two layers and the surface.

Here, the decay scale of an IW is considered to be much greater than the thickness of the mixed layer. For high frequency waves $(\omega>>f)$ and waves that will have multiple periods within a day, the vertical decay scale is roughly equivalent to the horizontal wavelength. Additionally, the surface boundary condition requires the vertical velocity to be zero at the surface of the ocean. For the two layer system with a density jump at the seasonal thermocline, the ratio of the average horizontal kinetic energy (HKE) of the mixed layer $(\mathrm{ML}),\left\langle U_{M L}^{2}\right\rangle$, to the stratified interior (SI), $\left\langle U_{S I}^{2}\right\rangle$, which is defined as the average over a wavelength of a wave, is

$$
\frac{\left\langle U_{M L}^{2}\right\rangle}{\left\langle U_{S I}^{2}\right\rangle}=2\left[(m H)^{2}+\left(1-\frac{\alpha^{2}}{\omega^{2}-f^{2}} g^{\prime} H\right)^{2}\right]^{-1}
$$

where $m$ is the vertical wave number in the stratified interior as defined by Eq. 3.6 using $N_{i}=N_{0}, H$ is the depth of the mixed layer, $\alpha$ is the horizontal wavenumber, $f$ is the Coriolis parameter, $g^{\prime}$ is reduced gravity. Here, $g^{\prime}=g \Delta \rho / \rho_{0}$, where $g$ is the gravitational 
constant, $\Delta \rho$ is the density jump of the seasonal thermocline as shown in the simple profile of Fig. 3-8, and $\rho_{0}$ is a reference density. There is a factor of two difference between the result here and the result in D78. However, this does not change the underlying physics of the problem and is most likely due to an assumption difference as the assumptions in D78 are not well detailed.

There are two squared terms on the right-hand side of Eq. 3.7, each with a clear physical significance. To explore each term it is useful to examine cases with and without the density jump at the seasonal thermocline. Given a density jump of $1 \mathrm{~kg} / \mathrm{m}^{3}$ and a mixed layer depth of $50 \mathrm{~m}$ the HKE ratio is shown as a function of wavenumber and frequency in Fig. 3-9a. A case with a mixed layer depth of $50 \mathrm{~m}$ and no density jump at the seasonal thermocline is shown in Fig. 3-9b. The difference between the two is shown in Fig. 3-9c. The horizontal velocity induced by an IW is proportional to the vertical derivative of vertical velocity $\left(\partial w^{\prime} / \partial z\right)$. A density jump will cause a discontinuity in the vertical derivative of vertical velocity, and thus a jump in horizontal velocity and HKW corresponding strong vertical shear at the seasonal thermocline. The relationship between increased shear, fine structure, and turbulence, and increasing stratification in the thermocline is well-documented (Gargett et al., 1981; Itsweire et al., 1993; Large et al., 1994; Pinkel and Anderson, 1997; Sun et al., 2013); however, IW mixing is an important ongoing research topic in oceanography.

The dispersion relation of the interfacial waves along the seasonal thermocline is given by the second set of terms of the right-hand side, here referred to as the "filter term," and can be written as $\omega^{2}=f^{2}+\alpha^{2} g^{\prime} H$. If we neglect the Coriolis term, the phase speed of waves along the seasonal thermocline is $\left(g^{\prime} H\right)^{1 / 2}$, which is the speed of gravity waves along an interface in a reduced gravity model. This curve is the dotted line shown in Fig. 3-9a and c. The HKE ratio is largest along the curve, and increases as frequency increases.

The density jump acts as a linear filter. It resonantly amplifies the wavenumbers and frequencies along the dispersion curve of the interfacial waves and suppresses others (Fig. 39c). A density jump has a natural frequency that can be excited by deep internal waves that interact with the jump (a famous real-world example of resonance is the Tacoma Narrows Bridge disaster). Specifically, the thermocline reflects certain sets of waves based on the strength and depth of the seasonal thermocline, as is shown by the negative HKE ratio difference above the interfacial wave dispersion curve. For large density jumps, such as the density jump at the surface of the ocean between atmosphere and ocean, the jump can be 

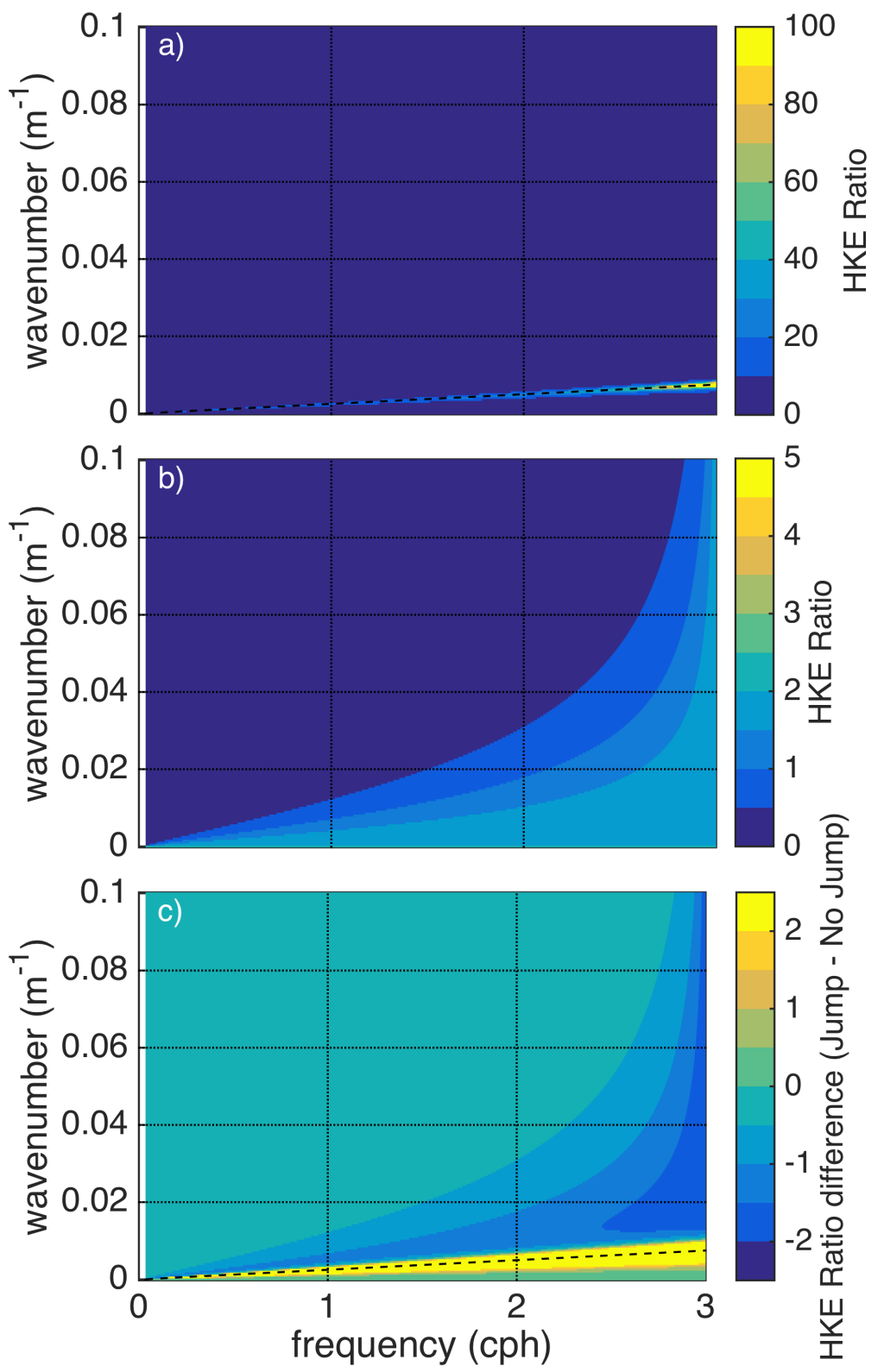

Figure 3-9: Wavenumber-frequency diagram of the horizontal kinetic energy (HKE) ratio of the mixed layer to the stratified interior as defined in Fig. 3-8 for (a) the case shown with a non-dimensional density jump (given as $\Delta \rho / \rho_{0}$ ) of 0.001 , (b) the case without a density jump at the seasonal thermocline, and (c) the difference between the case with the density jump in (a) and without (b) to show the effect of the idealized seasonal thermocline. 
thought of as a rigid lid that will reflect all waves. As the density jump strength increases more waves will be reflected. The $g^{\prime}$ term will dominate the filter term and the HKE ratio will approach zero.

Upward propagating IWs can cause resonant amplification of interfacial waves along the seasonal thermocline. Those excited waves decay into the mixed layer causing additional horizontal velocities. This is in addition to the horizontal velocities induced by waves that are able to decay through the mixed layer. Those waves are considered in the $(m H)^{2}$ term, here referred to as the "constriction term."

For waves with long vertical wavelengths relative to the depth of the mixed layer, the energy of the wave will be compressed to the thickness of the mixed layer (given the surface boundary condition). An increase in HKE in the mixed layer is expected (Fig 3-9b) for waves where $m H<1$. Since the HKE varies with the vertical derivative of vertical velocity, such that in the mixed layer $w^{\prime} \sim w(z=-H) / H$, deep mixed layers or waves with weak vertical velocities will correspond to small HKE.

Deep IWs propagate into the near-surface ocean, interact with the vertically-varying stratification, and can induce horizontal velocities in the mixed layer. This mechanism however, cannot cause mixing as it would not induce vorticity in the mixed layer (likewise one could consider that there cannot be a momentum flux divergence of the evanescent wave, which is necessary for turbulence). The central mooring data can be used to explore horizontal velocities as a function of frequency. Therefore, using the Garrett and Munk spectrum for IWs (hereafter GM; Garrett and Munk, 1972, 1975, 1979; Munk, 1981) in the stratified interior, the HKE ratio as a function of frequency can be estimated using numerical integration over wavenumber (Fig. 3-10). The details of the derivation are in Appendix B. There is an enhancement of HKE in the mixed layer compared to the stratified interior over

all frequencies. This is dominated by waves with decay scales greater than the thickness of the mixed layer. A peak in the HKE ratio occurs at the buoyancy frequency of the stratified interior.

\subsubsection{Observational evidence of D78 mechanism}

Using observations of horizontal velocities and calculated displacement in the ocean from the SPURS-I central mooring, the relationship between the HKE of the ML and SI is examined. In reality, on average, there is a relatively thin mixed layer atop a strongly stratified seasonal 


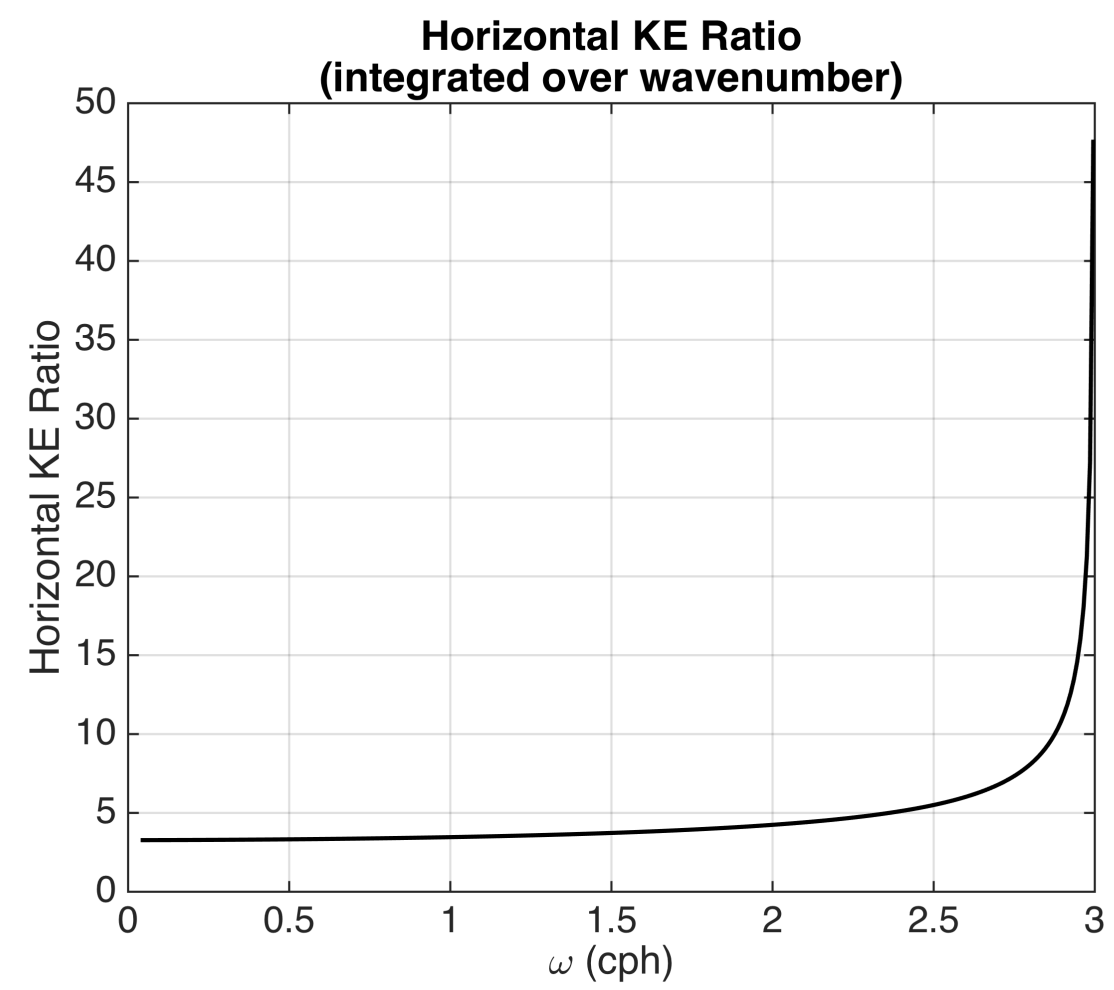

Figure 3-10: Horizontal kinetic energy ratio of the mixed layer to the stratified interior as a function of frequency, numerically integrated over wavelength, using the Garrett and Munk spectrum for IWs in the stratified interior. The horizontal KE ratio as a function of wavenumber and frequency is shown by Eq. 3.7. A $50 \mathrm{~m}$ deep mixed layer is used with a non-dimensional density jump (given as $\Delta \rho / \rho_{0}$ ) of 0.001 . 


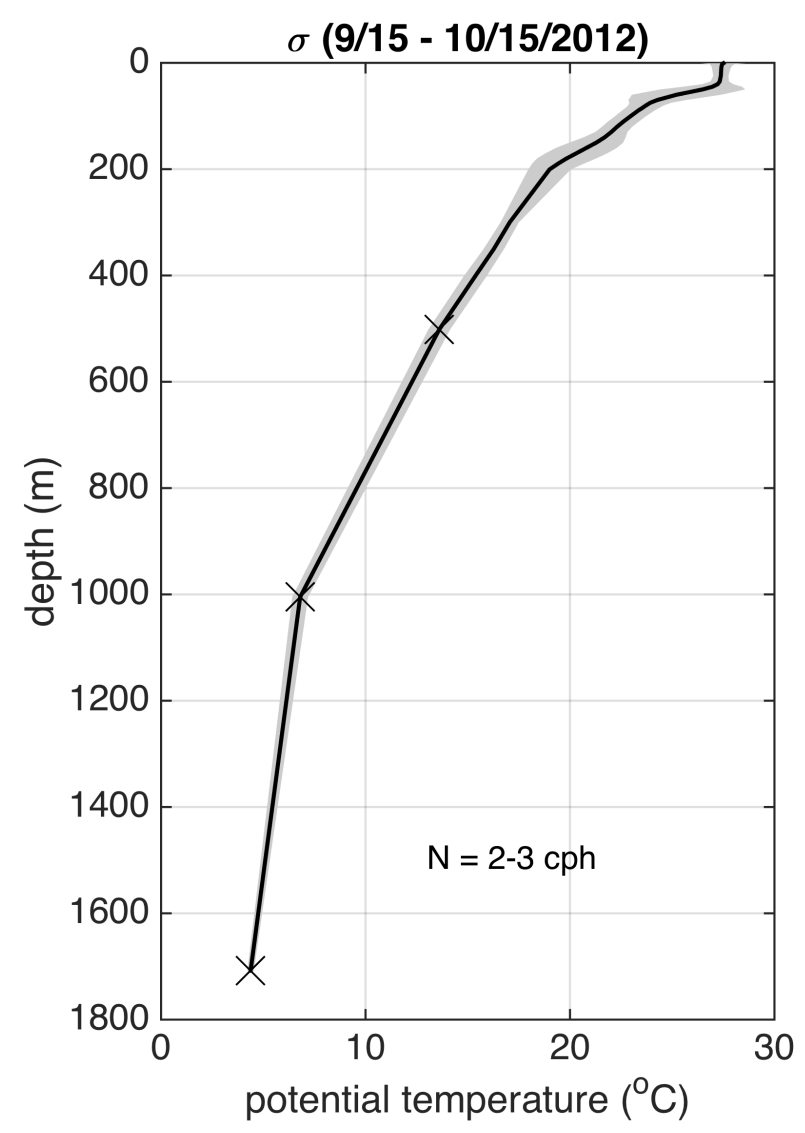

Figure 3-11: Average potential temperature profile from September 15 - October 15, 2012 from the SPURS-I mooring center at $25^{\circ} \mathrm{N}, 38^{\circ} \mathrm{W}$. The grey fill indicates the standard deviation of the average profile. The three x's indicate the depths of the deep temperature observations. Above 400 meters the mooring is well-instrumented.

thermocline (Fig. 3-11). The stratification slowly changes until it becomes relatively constant deep in the ocean, which for the SPURS-I region is about $1000 \mathrm{~m}$. For the time period of September 15 - October 15, the average profile from the SPURS-I mooring has a mixed layer about $50 \mathrm{~m}$ deep, and a seasonal thermocline (or region of strong stratification) between 50 - $150 \mathrm{~m}$. The numbers used to examine the linear model are estimated from the profile of potential temperature.

The velocity measurements from the ADCPs in the upper $400 \mathrm{~m}$ are hourly and the three VMCMs deep in the ocean provide one-minute observations (Fig. 3-12a). To provide better temporal resolution in the upper ocean, displacement is examined as the CTD measurements are every five minutes (Fig. 3-12b). Within the mixed layer there should not be an IW signal from displacement, $d_{\rho}$, since $d_{\rho}=w / N^{2}$, and the buoyancy frequency is identically zero in 
a mixed layer. However, just below the mixed layer the displacement signal is useful.

Deeper in the ocean $(1700 \mathrm{~m})$ the HKE spectrum matches GM quite well (using GM76 with unadjusted constants of the parameterization and buoyancy frequency of the deep ocean, $N_{0}$ ). However, the spectra flatten out at higher frequencies at shallower depths. There is also a statistically significant peak (at the $95 \%$ confidence level) at the buoyancy frequency of the deep ocean observed in the shallower VMCMs at approximately 500 and 1000m (Fig. 3-12a). This is also observed in displacement (Fig. 3-12b). Similar signals have been previously observed (e.g. Roth et al., 1981). In the linear model, the GM spectrum is used to define the stratified interior however, in actuality some of the energy of the resonantly excited waves with $\omega<N_{0}$ would also be expected to propagate deeper into the ocean. In addition, the ocean is non-linear and wave-wave interaction can cause the transfer of energy across frequencies (Müller et al., 2015). It is expected that a more diffusive peak at the buoyancy frequency is observed in the real ocean than the discrete peak in Fig. 3-10.

The statistically significant peak at the buoyancy frequency of the deep ocean is observed nearer to the surface in displacement (Fig. 3-12b). The highest frequency that can be explored with the ADCPs is $0.5 \mathrm{cph}$. The peak actually becomes more discrete for depths closer to the surface. Clear peaks in both HKE and displacement are observed between 2-3 cph, which is the buoyancy frequency of the deep ocean. The observations and spectral estimates generally agree with the simple model used here, including the existence of a peak in the HKE ratio at the buoyancy frequency of the deep ocean.

Although the observations point to deep IWs generating near-surface horizontal velocities, greater observations are needed with the direct intent to observe higher frequency variability and IWs to perform a more thorough analysis. The linear theory used here is instructive, but the ocean is not linear, especially concerning breaking IWs (Garrett and Munk, 1979). However, extending the linear model to include a diurnal warm layer will provide insight into the possible cause of the enhanced TKE dissipation by exploring when internal waves may induce a flow that generates instabilities and small scale turbulence.

\subsection{Inclusion of a diurnal warm layer}

The traditional conception of the ocean boundary layer is a mixed layer atop the stratified interior, precluding locally propagating high frequency IWs. However, during the daytime 

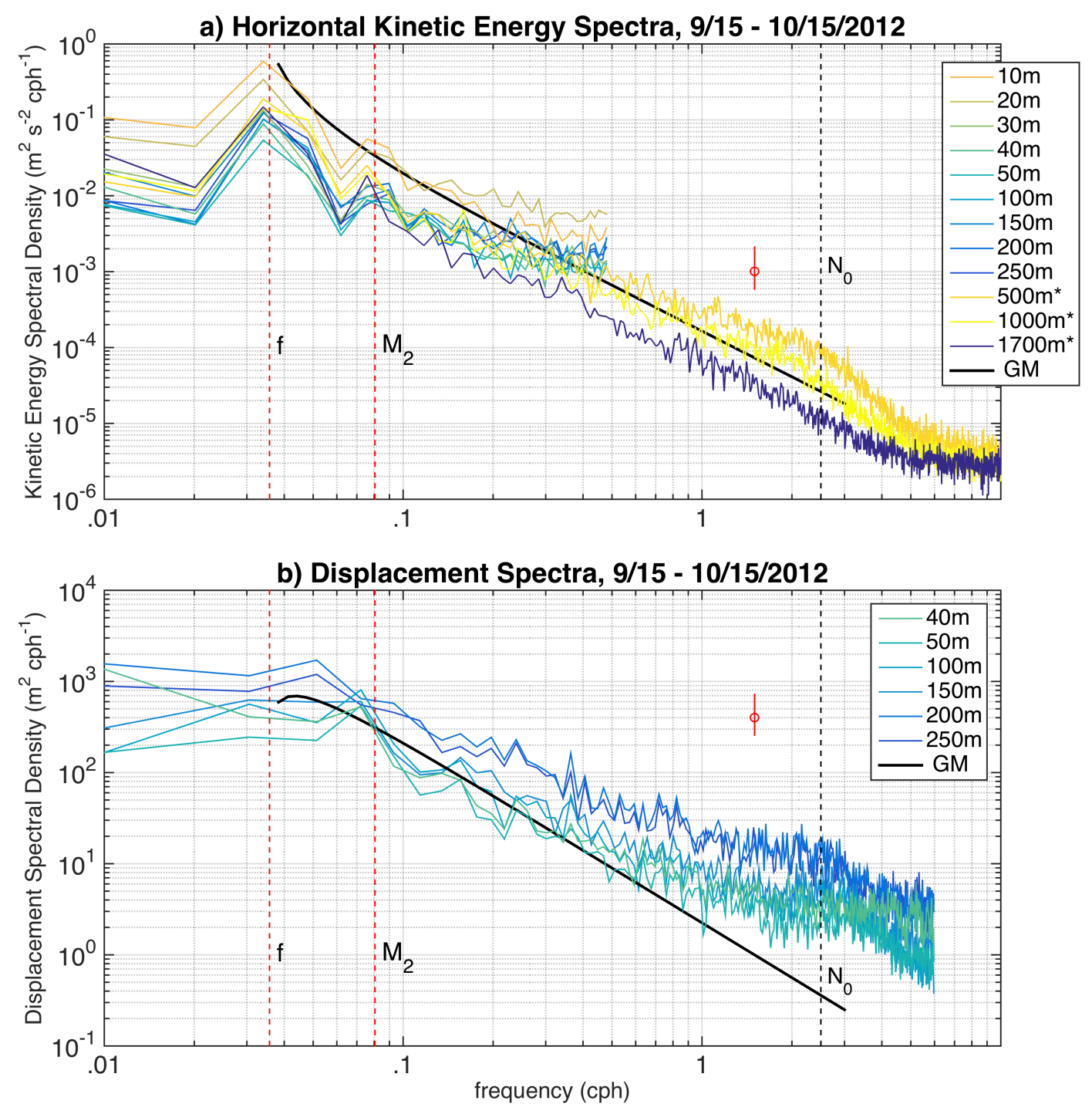

Figure 3-12: The (a) horizontal kinetic energy (HKE) spectral density and (b) displacement spectra for a range of depths from the SPURS-I central mooring from September 15 - October 15, 2012. The starred depths indicate deep VMCMs instead of ADCP measurements, which are every minute and hour, respectively. The shallowest displacement spectra is at the depth of the shallowest isopycnal that existed for the entire month period. The depth denoted for displacement is the average depth of the isopycnal over the one month time period. The red circle and vertical line shows the extent of the $95 \%$ confidence level on the spectral estimates. The vertical dashed lines indicate important frequencies. Note the peak in displacement spectra at the buoyancy frequency of the deep ocean, $N_{0}$. 
the near-surface ocean stratifies so that a stable layer exists atop the mixed layer (Fig. 3-8b). For reference, a $1^{\circ} \mathrm{C}$ change over the uppermost meter corresponds to a buoyancy frequency of $25 \mathrm{cph}$ and a weaker warming of $0.1^{\circ} \mathrm{C}$ over the upper 10 meters corresponds to a buoyancy frequency of $2.5 \mathrm{cph}$. IWs with long enough vertical decay scales can transport energy through the well-mixed layer into the surface diurnal warm layer. Here, a density jump of $1 \mathrm{~kg} / \mathrm{m}^{3}$ (a change in temperature of about $3^{\circ} \mathrm{C}$ ) is assumed at the seasonal thermocline at 50 meters depth. The diurnal warm layer (DWL) is assumed to be 5 meters deep, which is an average value based on observations. A composite of all days with observable warming was created. The DWL depth is estimated based on the greatest depth at which $0.1^{\circ} \mathrm{C}$ peak daily warming is observed in the composite.

The temperature variance of the nearest surface CTD from the SPURS-I mooring (0.75 m) reveals a pattern consistent with Farrar et al. (2007) that the largest temperature variance is observed on days with the weakest winds (Fig. 3-13). IWs are the most likely cause of the large temperature variance observed during the daytime, and the observations further demonstrate the importance of considering IWs within the DWL. The simple model used here will show that deep IWs can resonantly amplify IWs at certain frequencies and wavenumbers in the stratified near-surface ocean.

Starting with Eq. 3.5, a DWL can be included as a near-surface stratified layer with a constant buoyancy frequency as shown in Fig. 3-8b. The details of derivation are in Appendix B. The ratio of the HKE of the DWL, $\left\langle U_{D W L}^{2}\right\rangle$, to the HKE of the stratified interior, $\left\langle U_{S I}^{2}\right\rangle$, can be written as

$$
\frac{\left\langle U_{D W L}^{2}\right\rangle}{\left\langle U_{S I}^{2}\right\rangle}=\frac{2 n^{2} d^{2}+n d \sin (2 n d)}{2 \sin ^{2}(n d)}\left[m^{2} H_{*}^{2}+\left(\mu d_{*}-\frac{\alpha^{2}}{\omega^{2}-f^{2}} g^{\prime} H_{*}\right)^{2}\right]^{-1}
$$

where $n$ is the vertical wavenumber of the DWL (equivalent to $m$ in Eq. 3.6 with $N_{i}=N_{d}$ ), $d$ is the depth of the DWL, $m$ is the vertical wavenumber of the stratified interior $\left(N_{i}=\right.$ $\left.N_{0}\right), H_{*}$ is "equivalent thickness" of the remnant mixed layer (unstratified layer between the diurnal warm layer and seasonal thermocline), $\mu$ is the imaginary part of the vertical wavenumber of the remnant mixed layer, and $d_{*}$ is the "equivalent thickness" of the DWL. The two effective thicknesses are

$$
H_{*}=d\left(\cosh (\mu(H-d))+\frac{n \sinh (\mu(H-d))}{\mu \tan (n d)}\right)
$$




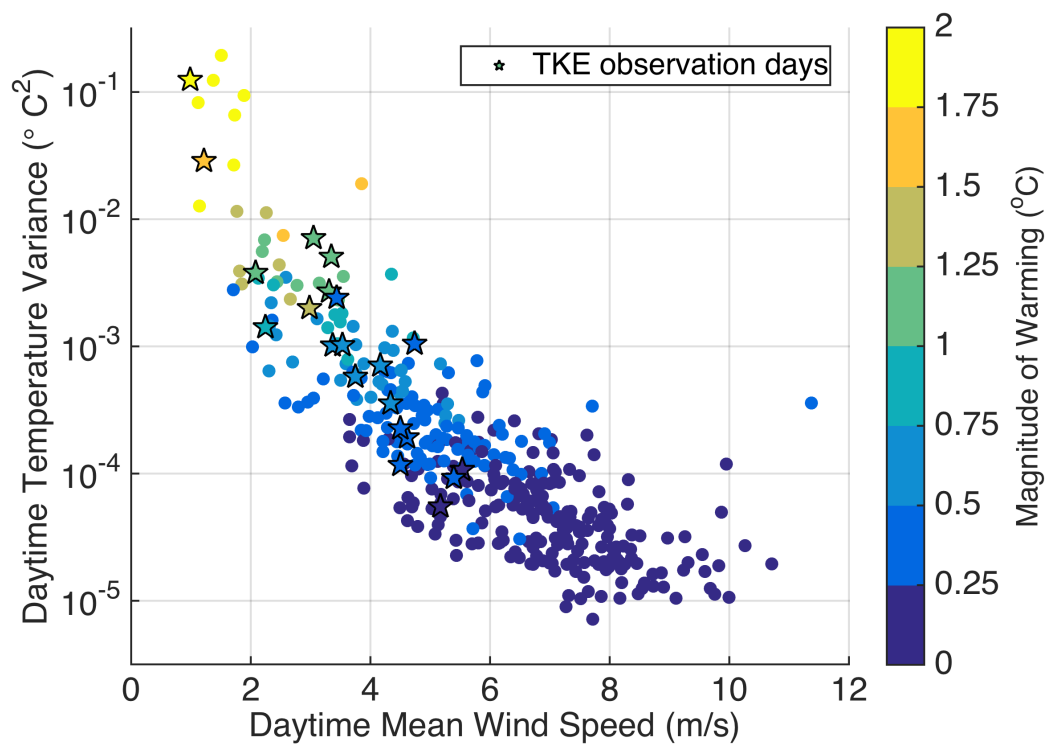

Figure 3-13: Daytime temperature variance (in log scale) at the $0.75 \mathrm{~m}$ CTD estimated over a day with a 4-hour high pass filter to remove the large diurnal amplitudes versus daily mean wind speed for the entire SPURS-I record. The The color indicates the magnitude of warming over the day. The days with observed high TKE dissipation are indicated as stars. As wind speed decreases, daytime temperature variances increases.

and

$$
d_{*}=d\left(\sinh (\mu(H-d))+\frac{n \cosh (\mu(H-d))}{\mu \tan (n d)}\right)
$$

where $H$ is the depth of the seasonal thermocline. The equivalent thicknesses are determined by the relative depths of the DWL and remnant mixed layer, the buoyancy frequency of the DWL, and the decay scale of the IW from the deep ocean. The equivalent depth will be referenced again when discussing resonant modes of the DWL. The HKE ratio is produced for comparison with Eq. 3.7 (the ratio without the DWL); however, it is more instructive to examine shear across the stratified diurnal warm layer in order to explore the possibility of a Richardson number (or shear) instability.

If the IW-induced shear can cause great enough shear across the DWL for the Richardson number $\left(\mathrm{Ri}=N^{2} / S^{2}\right)$ to drop below the critical value of 0.25 , the wave can break and turbulence can occur. The buoyancy frequency of the DWL is prescribed. The simple model can predict the shear across the layer. The maximum shear squared across the depth 
of DWL for a given frequency and wavenumber can be written as

$$
\operatorname{MAX}\left(\vec{u}_{z}^{2}\right)=\frac{A^{2} n^{4}}{\alpha^{2}} F_{\max }
$$

where

$$
F_{\text {max }}=\operatorname{MAX}\left[\left(\cos (n(z+d))-\frac{\sin (n(z+d))}{\tan (n d)}\right)^{2}\right]
$$

and

$$
A(\alpha, \omega)^{2}=8 d^{2}\left\langle U_{G M}^{2}\right\rangle\left(\frac{\omega^{2}}{\omega^{2}+f^{2}}\right)\left[m^{2} H_{*}^{2}+\left(\mu d_{*}-\frac{\alpha^{2}}{\omega^{2}-f^{2}} g^{\prime} H_{*}\right)^{2}\right]^{-1}
$$

$F_{\max }$ is a vertical oscillation term for propagating IWs in the DWL. All terms are already defined, except the $\left\langle U_{G M}^{2}\right\rangle$ term. An amplitude for the IWs in the stratified interior must be assumed, and the GM spectrum is used. Thus, the horizontal kinetic energy at a specific wavenumber and frequency from GM can be written as

$$
\left\langle U_{G M}^{2}\right\rangle=N_{0} b^{2} N \frac{\omega^{2}+f^{2}}{\omega^{2}} E(\alpha, \omega)
$$

where $N_{0}$ is the scaling quantity of the buoyancy frequency of at the top of the thermocline (3 cph), $b$ is the scaling quantity of the buoyancy scale depth $(1.3 \mathrm{~km}), N$ is the buoyancy frequency of the stratified interior, and $E(\alpha, \omega)$ is the non-dimesnional energy spectrum as a function of wavenumber and frequency (see Garrett and Munk, 1975).

Examining the spectral density of shear squared in the diurnal warm layer reveals the key result that larger values of shear occur with increasing stratification in the DWL (Fig. 314). The largest values occur at lower frequencies and wavenumbers (long period, long wavelength waves). When the stratification of the DWL increases to a value greater than the deep ocean, the vertical wavenumber of a wave within the DWL will be smaller than the vertical wavenumber of the same wave in the SI. The vertical wavelength of an IW is shorter in the DWL than in the SI. In a sense, a greater range of frequencies and horizontal wavenumbers can "fit" in the DWL. Each mode will have a different equivalent DWL depth and remnant mixed layer thickness that relate to the speed of the waves. The waves along the seasonal thermocline are also inconsequential with respect to generating shear across the DWL. 

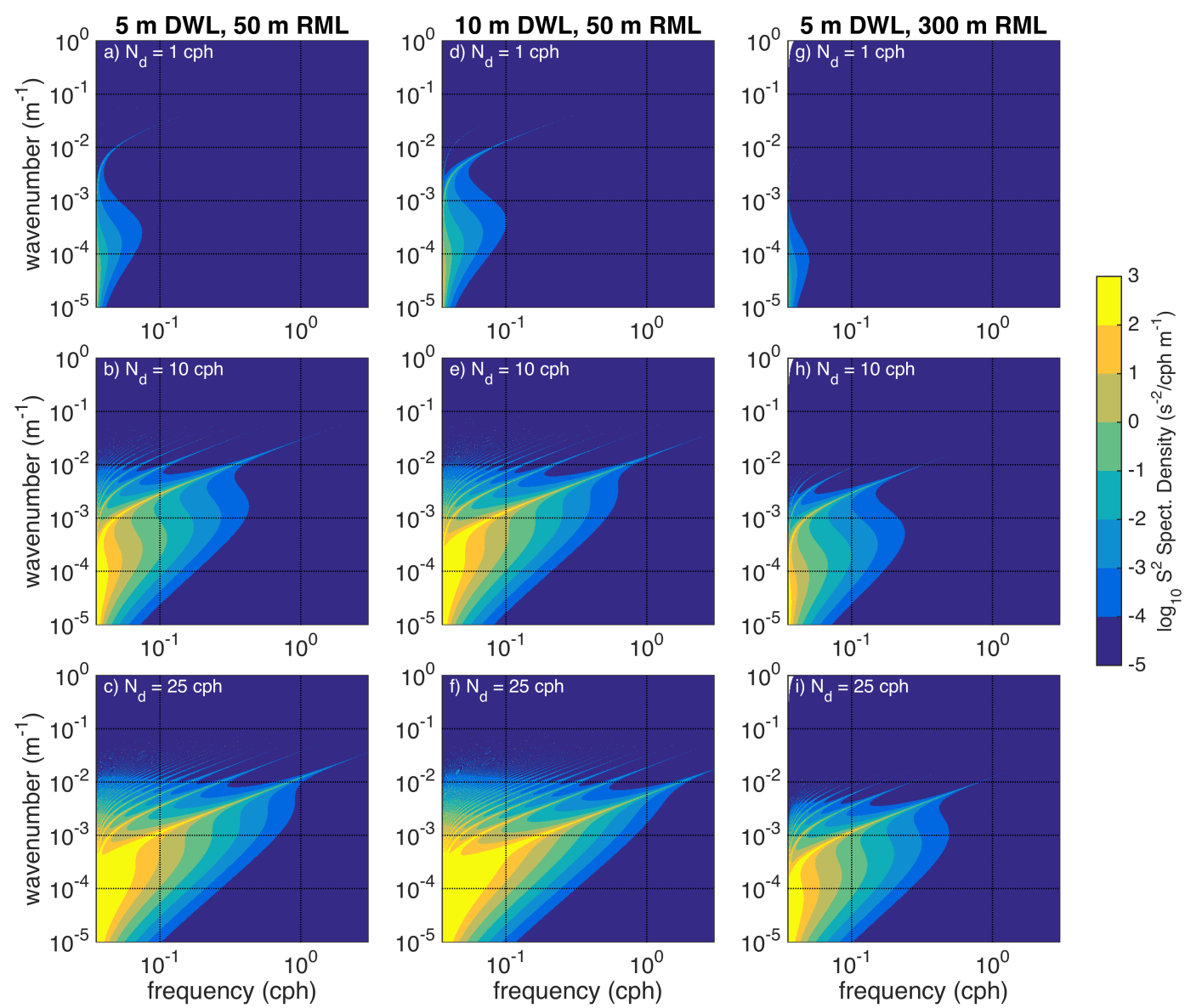

Figure 3-14: Shear squared calculated from the simple model with a diurnal warm layer with an $\mathrm{N}_{\mathrm{d}}$ of $(\mathrm{a}, \mathrm{d}, \mathrm{g}) 25 \mathrm{cph}$, (b,e,h) $10 \mathrm{cph}$, and (c,f,i) $1 \mathrm{cph}$. For all panels, a density jump of 1 $\mathrm{kg} / \mathrm{m}^{3}$ is used at a seasonal thermocline. For the first column, a seasonal thermocline depth of $50 \mathrm{~m}$ with a diurnal warm layer depth of $5 \mathrm{~m}$ is used. For the second column, the diurnal warm layer depth is doubled to $10 \mathrm{~m}$. For the third column, the seasonal thermocline depth is increased to $300 \mathrm{~m}$. Note Fig. 3-9 is similarly shows results in a wavenumber-frequency space however, here a log-log scale is use to highlight low frequencies and wavenumbers. 
The depth of the DWL, depth of seasonal thermocline, and buoyancy frequency of the DWL are varied to examine the relative importance of each input. The largest changes in spectral density occur with changing the buoyancy frequency of the DWL, assuming plausible inputs of all variables (Fig. 3-14a,b,c, for example). In addition to the increase in spectral density, the dispersion curves of the resonant modes (lines that extend into higher frequencies and wavenumbers) become less steep corresponding to decreases in phase speed. Each successively less steep line represents a resonant mode in the DWL. In the case of a much deeper seasonal thermocline (Fig. 3-14b,h), the magnitude of the shear spectral density is noticeably reduced. Varying the depth of the diurnal warm layer changes the steepness of dispersion curves of the resonant modes and also the magnitude of the shear squared spectral density (Fig. 3-14b,e).

To estimate the shear due to a spectrum of IWs in the DWL, the spectral density is integrated over wavenumber and frequency (Fig. 3-15a). This requires the assumption of random phasing across the spectrum of waves. As the buoyancy frequency of the DWL increases, so does the mean-squared shear. It is slightly counterintuitive that shear increases with increased buoyancy frequency since a more stable environment is expected. However, this is exactly the case that allows IWs that reach into the DWL to generate strong shear across that layer.

\subsubsection{The diurnal warm layer as a waveguide}

The source of IWs observed by Walsh et al. (1998), Farrar et al. (2007), or Hodges and Fratantoni (2014) is plausibly the deep ocean. What becomes apparent through this simple model is that the diurnal thermocline can act as a waveguide trapping waves between the surface and the top of the remnant mixed layer. For strong stratification, the diurnal thermocline will qualitatively be much like the density jump at the seasonal thermocline. Thus, interfacial waves will propagate along the thermocline.

If the source of the waves is the deep ocean, then the highest frequency waves will be at the buoyancy frequency of the deep ocean. Waves with large enough decay scales can propagate through the remnant mixed layer and become trapped in the DWL. Waves propagating along the DWL can create a surface signal. This is a different mechanism for the surface signal of IWs than waves along the thermocline generating a surface signal due to regions of convergence and divergence (Alpers, 1985; Walsh et al., 1998), but can only 
be expected when a near-surface stratified layer exists (either a diurnal warm layer or fresh water lens, Soloviev et al., 2015).

\subsubsection{Potential turbulence generation mechanism}

Once the predicted Richardson number drops below 0.25 , it is expected that the flow will become unstable and generate small scale turbulence. Linear theory can predict when Ri may be less than 0.25 . It is likely a necessary, but not sufficient, condition for instability as the flow here is time dependent. However, for this study, we are not considering time dependency. Given the estimate of mean-squared shear (the square root is a standard deviation), assuming zero mean and a Gaussian distribution of shear, the possibility of the predicted shear causing $R i<0.25$ can be determined (Fig. 3-15b). The spectral density is integrated over wavenumber and frequency assuming random phasing across the spectrum of waves. The probability of the IW shear causing $R i<0.25$ is the chance that a the shear based on a Gaussian distribution is $\sqrt{4}$ times the buoyancy frequency of the DWL (considering two tails and both positive and negative shear).

At low values of $N$ for the DWL $\left(N_{d}\right)$, shear can more easily cause turbulence as stronger stratification will require stronger shear to generate a shear instability. The minimum chance of IW shear causing $R i<0.25$ occurs a $N_{d}=4 \mathrm{cph}$. For the sake of comparison, a $4 \mathrm{cph}$ buoyancy frequency corresponds to $0.05^{\circ} \mathrm{C}$ temperature change over $1 \mathrm{~m}$ (a very small diurnal warming value). We have not considered mean flow within the DWL. The DWL is a sheared layer that has been shown to be marginally stable (Kudryavtsev and Soloviev, 1990) and therefore, even relatively small shear associated with IWs may cause instability and generate turbulence.

After $4 \mathrm{cph}$, the fractional chance increases with $N_{d}$ steadily until around $30 \mathrm{cph}$ (corresponding to $2.7^{\circ} \mathrm{C}$ warming over $\left.1 \mathrm{~m}\right)$. For relatively strong diurnal warming events $(20$ - $40 \mathrm{cph}, 1-5^{\circ} \mathrm{C}$ over $1 \mathrm{~m}$ ), the fractional chance of a shear instability is about $10 \%$. This means that if IWs are constantly propagating into the near-surface ocean, it is quite plausible that shear instability associated with IWs can provide a source of turbulence and TKE dissipation.

There is more than enough energy in the IW field to explain all of the observed TKE dissipation. Integrating the energy in the internal wave field in GM spectrum across wavenumber and frequency space and using an average dissipation in the upper 30 meters of the ocean 

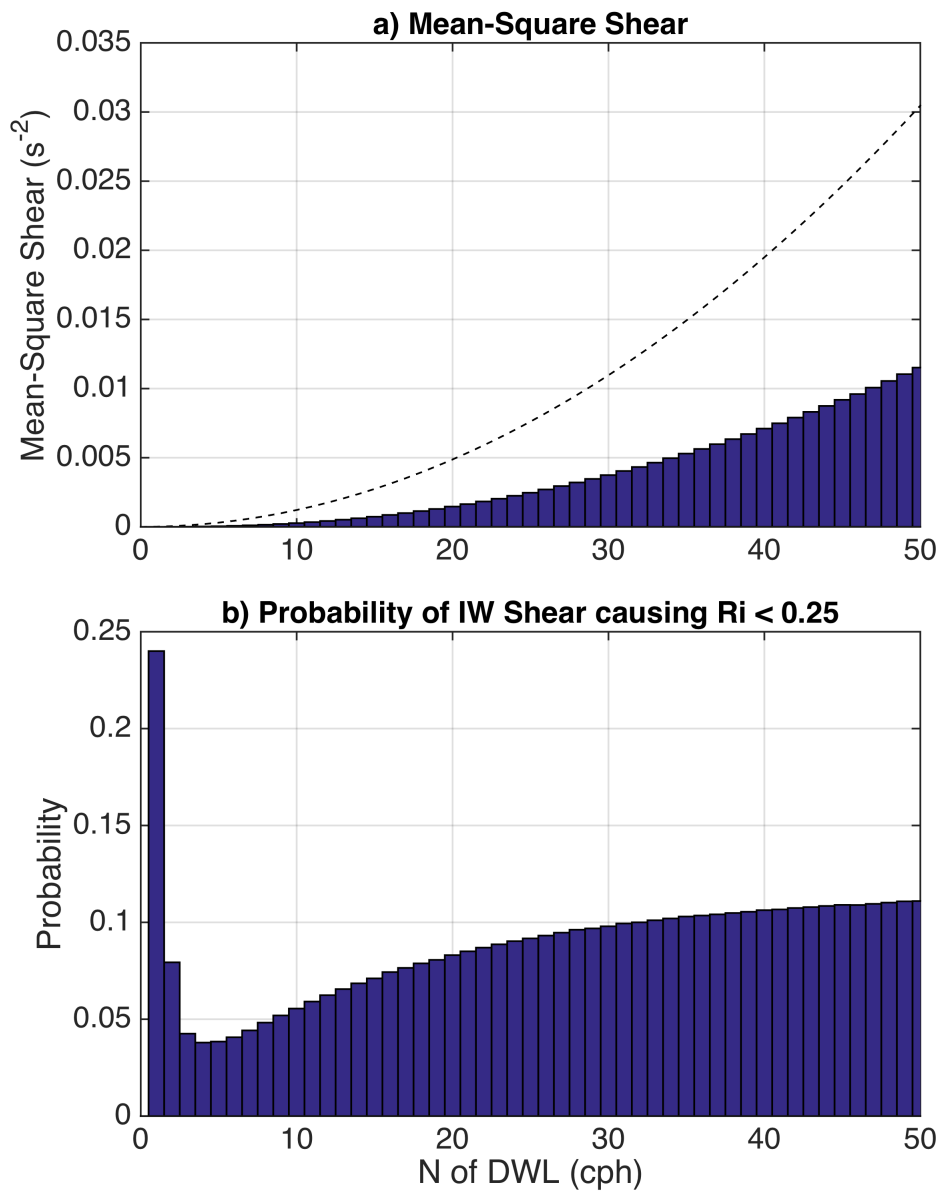

Figure 3-15: The (a) mean-square shear (over all wavenumber and frequency space) and (b) the probability of shear causing a $\mathrm{Ri}<0.25$ assuming zero mean shear and using the mean-square shear as the variance of a Gaussian probability function. This setup includes a density jump of $1 \mathrm{~kg} / \mathrm{m}^{3}$ used at a seasonal thermocline depth of $50 \mathrm{~m}$ with a diurnal warm layer depth of $5 \mathrm{~m}$ (the first column of Fig. 3-14). The dashed line is (a) is 4 times $\mathrm{N}^{2}$ to show the value at which $\mathrm{Ri}=0.25$. 


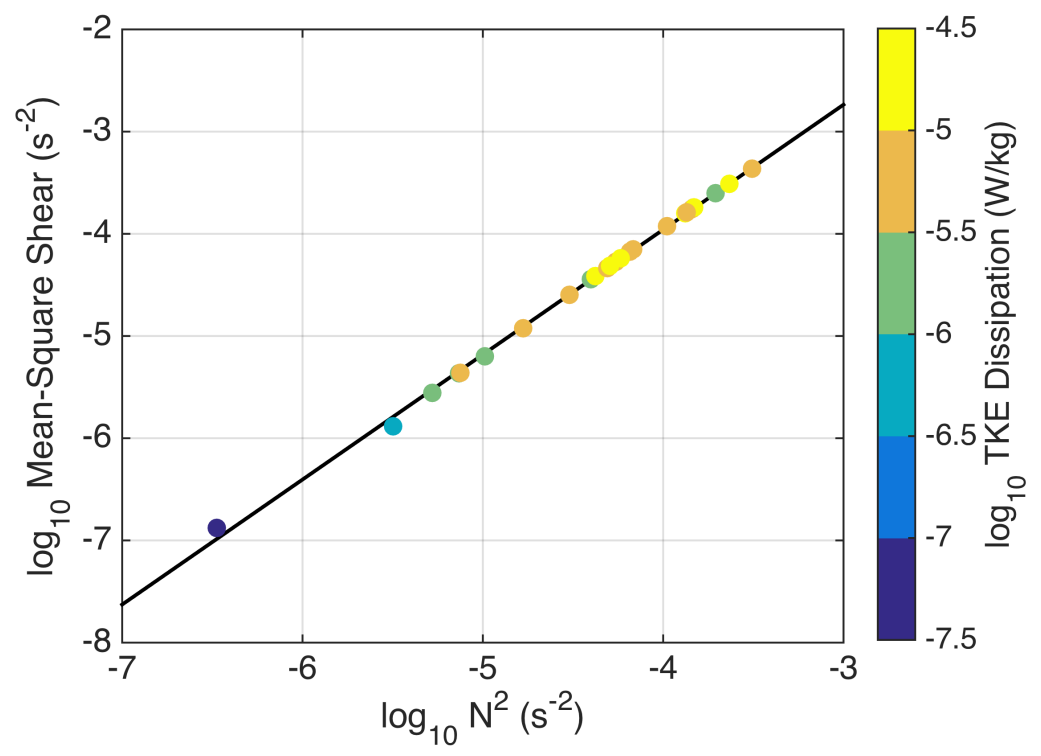

Figure 3-16: Buoyancy frequency of observed days with during SPURS-I cruises dailyaveraged over the upper 10 meters (only during daylight hours) from the Slocum gliders versus the fractional area of wavenumber-frequency space estimated from the simple IW model. The color indicates the daily-average TKE dissipation from the gliders over 10 meters. The black line is the least squares best fit line.

during the daytime under enhanced TKE dissipation conditions provides a dissipation timescale of 30 days. This means it would take a month of continuous enhanced dissipation in the upper ocean to convert all of the energy in the internal wave field to TKE dissipation. This assumes no source of energy input into the internal wave field and continuous enhanced TKE dissipation. Both assumptions provide a conservative estimate of the dissipation time-scale. For instance, two constant sources of internal waves are the tidal forcing from the moon and topographically generated internal waves due to ocean currents (Garrett and Munk, 1979). Additionally, the TKE dissipation appears to start as soon as the upper ocean stratifies and continues until the net heat flux causes the ocean to cool and convection ensues (Fig. 3-1). The simple model reveals that only relatively small stratification is required in the DWL to see strong enough shear from IW to cause a shear instability.

Sethuraman (1980) found that persistent breaking atmospheric IWs in stable layers over the ocean significantly increased the turbulence (TKE dissipation by almost two orders of magnitude). A similar mechanism is likely occurring in the ocean boundary layer. Using the observations (Fig. 3-3), the predicted mean-squared shear from the simple model (using 
a $5 \mathrm{~m} \mathrm{DWL}$, and $50 \mathrm{~m}$ deep seasonal thermocline) is compared to the observed buoyancy frequency (Fig. 3-16). As $N_{d}$ increases so does the the mean squared shear, corresponding with larger values of TKE dissipation. Ultimately, the connection between all the days with higher than expected TKE dissipation during the daytime is a stabilizing heat forcing from solar radiation and stably stratified near-surface ocean, including observed IWs.

\subsubsection{Implications for mixing}

Although enhanced TKE dissipation is observed during the daytime and large shear is predicted by the simple model of internal waves proposed here, it seems unlikely that the large dissipation would imply enhanced mixing (i.e. vertical turbulent diffusivity) during the daytime. First, large mixing would wipe out the strong near-surface temperature gradients associated with DWLs. Strong gradients persist throughout the day indicating mixing is suppressed under low wind speed conditions with large warming magnitudes. This is also consistent with one-dimensional ocean models being able to reproduce the temperature signal of the near-surface ocean, but not the dissipation signal (Chapter 2). Second, a turbulent diffusivity can be calculated using the observed TKE dissipation and estimate of the buoyancy frequency (Osborn, 1980). The diffusivity estimated during the observed enhanced dissipation events are near molecular levels, which is consistent with background levels needed in the Kantha-Clayson model to reproduce the large warm magnitudes on very calm days (Chapter 2). However, described here is a mechanism for energy from the deep ocean to be transferred to and dissipated within the near-surface ocean.

\subsection{Summary and Conclusion}

The observations imply that the mechanism for the observed enhanced TKE dissipation during the daytime occurs under stabilizing surface forcing and requires a stratified near-surface ocean. Moreover, the observational evidence supports the hypothesis that IWs (propagating from the deep ocean) interact with the diurnal warm layer causing enhanced shear across that layer and, subsequently, enhanced TKE dissipation.

The conclusions of the chapter are:

1. Conditions in the upper ocean during the daytime are favorable for IWs, and the observed higher than expected TKE dissipation is associated with stabilizing surface 
forcing due to solar heating.

2. IWs are likely an important characteristic of the oceanic stable boundary layer, much like in the atmospheric stable boundary layer.

3. IWs propagating from the deep ocean can cause large horizontal velocities in the mixed layer observed through spectral estimates of horizontal velocity.

4. The addition of a diurnal warm layer substantially changes the propagation characteristics of waves from the deep ocean, and can cause shear across the diurnal warm layer associated with a $\mathrm{Ri}<0.25$.

5. Increasing stratification in the diurnal warm layer is associated with larger TKE dissipation values, and larger predicted shear caused by upward propagating IWs.

While the linear IW model is useful to understand the physics of the problem at hand, it comes with limitations. This analysis ignores shear present across the DWL due to momentum from the wind trapped by the stable stratification near the surface (Chapter 2). A simple analytical approach taking into account the shear layer is not possible. It is likely important and contributes to the instability and non-linearity near the surface, and is saved for a later study. Substantial questions still remain about the mixing efficiency (i.e. the relation of Ri to TKE dissipation) of the ocean in stratified sheared flow (Riley et al., 2003; Salehipour et al., 2015). Additionally, the model can predict when the flow will potentially cause an instability, but not the details of non-linearities that are necessary for the onset of turbulence.

It is important to briefly consider how IWs can generate a turbulent flow. First, lowfrequency low vertical amplitude near-inertial waves can propagate into the near-surface and enhance horizontal shear. The enhanced horizontal shear can reduce the Richardson number enough to create a shear instability. This is the case considered here. Interactions with critical layer can cause the transfer of momentum from IWs to the mean flow, thereby creating turbulent regions (Booker and Bretherton, 1967; Thorpe, 1975). Booker and Bretherton (1967) regard this mechanism as potentially extremely important to the transfer of momentum in the ocean. Also, reflection at the sea surface interface can change the characteristics of the wave if the surface is not perfectly flat, which is generally the case in reality. The exact turbulence generation mechanism will depend upon the frequency and amplitude of 
the IW. There have been some studies of wave motions in time varying stratification and their effect on the boundary layer (e.g., Kelly, 1967; Rotunno, 1977), but more detailed studies are needed.

IW propagation into a region with varying stratification that changes by order of magnitudes over tens of meters creates an incredibly complex situation. In addition, the time-scale of the change in stratification is similar to the time-scale of the IWs, near-inertial waves and internal tides making it challenging to distinguish the different phenomena. Sutherland and Yewchuk (2004) show that IWs can propagate through weakly stratified patches, which can occur between the seasonal and diurnal thermoclines. Mathur and Peacock (2009) explore IW packets propagating through complex stratification and find waves rarely continue as one beam through rapidly changing stratification. This presents a challenge for analytical solutions, but the problem can be done numerically. The stratification in this problem changes rapidly in space and time, and will be important to consider in future studies.

Just as similarity theory (characterizing the boundary layer by surface fluxes, a length scale, and distance from the boundary) does not appear to be relevant for the atmospheric stable boundary layer (Mahrt, 2014), Chapter 2 shows that similarity theory likely does not work for the ocean under stable conditions, when diurnal warm layers are expected. Greater focus on the importance of properly resolving the stable ocean boundary layer is needed, with less focus on parameterized mixing schemes based on theory that has been shown to be inappropriate under such conditions since the mixing may not be solely surface forced (such as the K-Profile Parameterization of Large et al., 1994). Better theory requires greater observations of the near-surface under stabilizing conditions, including currents, accurate measurements of penetrative solar radiation, and surface wave characteristics. Properly resolving IWs in the near-surface ocean also present an interesting modeling problem. The community is beginning to explore the near-surface ocean with high-resolution numerical models (e.g. Soloviev and Lukas, 2014; Soloviev et al., 2015), and stable layers in the ocean can be explored using these models.

The conclusions of this study are limited by the observations. The days with observed high TKE dissipation are low wind speed days (Fig. 3-13). The SPURS-I microstructure observations are limited to relatively low wind speed days. More observations are needed under a variety of conditions to consider the ubiquity of the results of this study. However, a clear, salient conclusion is that IWs are an important characteristic of the near-surface 
ocean that should not be ignored. 


\section{Chapter 4}

\section{Submesoscale lateral mixing by diurnal warm layer shear}

\subsection{Introduction}

At the air-sea interface, turbulent and longwave radiative fluxes generally cool and destabilize the ocean surface, while incoming solar radiation stabilizes the upper ocean during the daytime. Wind stress acting on the ocean generally transfers momentum from the atmosphere to the ocean, generating mechanical mixing. However, when incoming solar radiation heats and stabilizes the upper ocean, and creates a diurnal warm layer, the momentum can become trapped in this near-surface stable layer. As momentum builds in this relatively thin layer, it generates shear over the depth of the diurnal warm layer (hereafter abbreviated as DWL).

The concept of momentum trapped by stable stratification is not new. A thin nearsurface layer moving atop the rest of the ocean due to the high-temperature gradient was noted as "slippery seas" by Woods (1968). Price et al. (1986) dubbed this mechanism the "diurnal jet." Kudryavtsev and Soloviev (1990) observed this momentum trapping and found the velocities to be on the order of $0.1 \mathrm{~m} / \mathrm{s}$. During periods of low wind speed and high solar insolation the upper few meters of the ocean can warm and then cool several degrees over the course of a diurnal cycle (Gentemann et al., 2008), and the DWL shear can be trapped in the upper few meters of the ocean. Throughout this paper, the near-surface current caused by momentum trapped by the stable stratification of diurnal warming is referred to as DWL 
shear or the DWL current.

Near-surface freshening coincident with the diurnal cycle of temperature was observed on two days during a cruise aboard the R/V Knorr (September 28 and 29, 2012), and another day in the spring of the following year (April 29, 2013). All three days are associated with fairly strong diurnal warming. The freshening was unexpected as evaporation associated with turbulent fluxes at the surface of the ocean should cause a slight increase in salinity (Drushka et al., 2014). Neither local precipitation nor vertical mixing can explain the freshening, leading us to suspect that the shallow freshening was from horizontal advection by the DWL current.

The data were collected as part of the initial Salinity Processes in the Upper ocean Regional Study, or SPURS-I, field campaign, which focused on the climatological surface salinity maximum in the North Atlantic Ocean (centered at $25^{\circ} \mathrm{N}, 38^{\circ} \mathrm{W}$ ) with three associated month-long cruises. The field campaign was focused on the physical processes affecting sea surface salinity at a range of temporal and spatial scales. SPURS-I provided high temporaland vertical-resolution observation of the near-surface ocean from a variety of instruments deployed during the cruises. Understanding the salinity variability of the near-surface ocean on all time-scales is also important for accurate estimates of salinity from space, such as Aquarius (Melnichenko et al., 2014) and SMOS (Kerr et al., 2010).

$\mathrm{Yu}(2011)$ showed the dominant terms in the seasonal salt budget for the mixed layer in the SPURS-I region are advection and mixed layer entrainment, not surface fluxes. However, on shorter time-scales the dominant terms are less clear. Advection specifically associated with the diurnal warm layer current may be an important source of horizontal variability and transport in the ocean on shorter times scales.

The importance of resolving diurnal scales is becoming increasingly clear. The inclusion of a diurnally-varying sea surface temperature (compared to a daily-average or temperature below the diurnal warm layer) in air-sea turbulent flux calculations has been shown to dramatically change the instantaneous fluxes by up to 100\%, as well as annually-averaged flux by up to $10 \%$ in the tropics (Clayson and Bogdanoff, 2013; Weihs and Bourassa, 2014). Seo et al. (2014) argued that including the diurnal cycle is important to properly resolve the onset and intensity of the Madden-Julian Oscillation. In the upper ocean, the descent of the DWL at night has been shown to be a mechanism for the onset of turbulence and mixing deeper in the ocean (Smyth et al., 2013). Clayson et al. (2016b) detail unexpected enhanced 
dissipation of turbulent kinetic energy and thermal variance observed during SPURS-I field campaign during the daytime associated with diurnal warming. The cause of the enhanced dissipation is explored in Chapter 2 and 3.

The importance of the DWL current is explored here. The observed freshening, although it cannot be directly linked to the DWL current, motivated the mechanism described in this paper. Shear associated with the diurnal warm layer, which occurs over a fractional depth of the mixed layer, horizontally displaces water near the surface during the daytime, converting horizontal gradients into vertical ones At night, convection vertically mixes the horizontally displaced water throughout the (deeper) mixed layer. The mechanism is a type of shear dispersion associated with vertical shear and follows the concepts of Ferrari and Young (1997). This cycle of advection and then vertical mixing is a mechanism for submesoscale lateral diffusion on the scale of $1-10 \mathrm{~km}$ within the mixed layer.

Section 2 details the field campaign and observations used in this study. A discussion of the observed near-surface freshening is provided in section 3, with examination of surface forcing such as precipitation. A simple model to explain the effective lateral mixing associated with daytime differential surface advection followed by nighttime vertical mixing is presented in section 4, including diffusivity estimates. The three days with observed freshening are investigated using the simple model in section 5, with a focus on additional implications of diurnally-varying advection in the near-surface ocean. Conclusions and a discussion with global perspectives are provided in section 6 .

\subsection{Data}

There were three cruises associated with the SPURS-I field campaign: fall of 2012 ("deployment"), spring of 2013 ("midterm"), and fall of 2013 ("recovery"). Near-surface freshening was observed on three different days, including two during the deployment cruise with 21 days on-site at the SPURS-I study area from September 15 - October 5, 2012. During the deployment cruise, the heavily instrumented central mooring with accurate meteorological measurements for air-sea fluxes (Farrar et al., 2015) was deployed.

The SPURS-I central mooring at $24.5^{\circ} \mathrm{N}, 38^{\circ} \mathrm{W}$ had surface and subsurface components. The surface component included an IMET system (Hosom et al., 1995; Colbo and Weller, 2009), providing one-minute meteorological data for wind speed and direction, shortwave 
and longwave radiation, precipitation, and surface temperature and salinity. The mooring had three different rainfall sensors. Here, a net surface heat flux is calculated similarly as in Chapter 2:

$$
Q_{N}=Q_{L}+Q_{B}+Q_{P}+Q_{L W}+Q_{S W}
$$

where $Q_{N}$ is the net surface flux, $Q_{L}$ is the latent heat flux, $Q_{B}$ is the sensible heat flux, $Q_{P}$ is the heat flux due to temperature difference between the sea surface and precipitation, $Q_{L W}$ is the outgoing longwave radiation, and $Q_{S W}$ is the incoming shortwave radiation, and a positive flux is into the ocean. The turbulent fluxes are estimated from the COARE 3.0 algorithm (Fairall et al., 2003). The mooring's subsurface component included CTD measurements at $0.75 \mathrm{~m}$ and $2.1 \mathrm{~m}$, used in this paper. A point current meter provided hourly currents at $3.0 \mathrm{~m}$, and an upward facing $\mathrm{ADCP}$ at $18 \mathrm{~m}$ provided hourly current observations of the near-surface, but only to $3.55 \mathrm{~m}$, shy of the shallow depths explored here. The data used here are the same data used in Chapter 2 and 3 and Farrar et al. (2015).

In addition to the central mooring and to obtain better spatial coverage of the SPURS-I study area, Liquid Robotics Wave Gliders (Daniel et al., 2011) were deployed during the field campaign, and remained in the water for a year-long mission (other than a few days during the midterm cruise for servicing). Three Wave Gliders moved around a $1^{\circ} \mathrm{x} 1^{\circ}$ box near the central mooring in repeating paths to provide good temporal and spatial coverage. The instruments have surface and subsurface components spanning 6 meters in the vertical. The surface component is equipped with meteorological instrumentation, solar panels, and a CTD on the bottom side, nominally at $0.3 \mathrm{~m}$. The subsurface component is at $6 \mathrm{~m}$, and it was also instrumented with a CTD.

Two Webb-Teldyne Slocum gliders were deployed during the deployment and midterm cruises, and data are available for two of the three days with observed near-surface freshening. A total of 3,692 profiles around the mooring were conducted over 3 days of the two cruises. The gliders were equipped with Rockland Scientific MicroRiders to measure microstructure. The microstructure data are the focus of Clayson et al. (2016b) and Chapter 2. The gliders were ballasted light in order to better observe the near-surface ocean, and kept close to the mooring. The data are used to examine near-surface salinity near the mooring.

In addition to the assets deployed during the field campaign, the shipboard temperature 
and salinity data are also used. A SeaBird SBE45 intake for the system aboard the $\mathrm{R} / \mathrm{V}$ Knorr is mounted in the bow, nominally at 5 meters. Data are available for the two days with observed freshening during the cruise period, and only used when the ship is moving. Additionally, to augment the precipitation data from the mooring, especially with the intermittent and spatially inhomogeneous nature of precipitation, two satellite-based products are used. The NOAA Climate Prediction Center Morphing Technique (CMORPH) Global Precipitation Analysis bias-corrected 3-hourly quarter degree Version 1.0 product (Joyce et al., 2004) and daily 2.5 degree Global Precipitation Climatology Project (GPCP) Version 2.2 Combination product (Adler et al., 2003) are utilized to explore rainfall as a possible source of the near-surface freshening.

\subsection{Near-surface freshening}

During the SPURS-I deployment cruise there were two large-amplitude diurnal warming days without measurable rainfall at the mooring on September 28 and 29, 2012 (hereafter referred to as September 28 and September 29). Near-surface freshening that followed the diurnal cycle is observed on those two days. Freshening was observed at the mooring in the $0.75 \mathrm{~m}$ salinity record, and much more weakly in the $2.1 \mathrm{~m}$ record (Fig. $4-1 \mathrm{~d}, \mathrm{~h}$ ). A 30 minute moving average was performed on mooring data and an hour moving average on the Wave Gliders to remove high frequency variability (likely associated with internal waves). The maximum freshening was 0.08 psu on September 28 and 0.35 psu on September 29.

The freshening begins around peak warming for each day. While two cases are not enough to demonstrate a connection, the freshening almost exactly follows the diurnal cycle of temperature, and may be connected to phenomena such as DWL shear. Vertical mixing cannot explain the freshening as the lower salinity is not observed in the water column. Thus, only surface fluxes or advection, likely associated with DWL shear, can explain the freshening. During those two days, no rainfall was measured by any of the three sensors (Table 4.1), nor was rainfall measured at the ship. A third day during the mooring record, April 28, 2013, also had freshening coincident with the diurnal cycle (Fig. 4-2d), herein referred to as April 28. The freshening on that day was $0.48 \mathrm{psu}$. There was no precipitation recorded by the mooring on that day. 

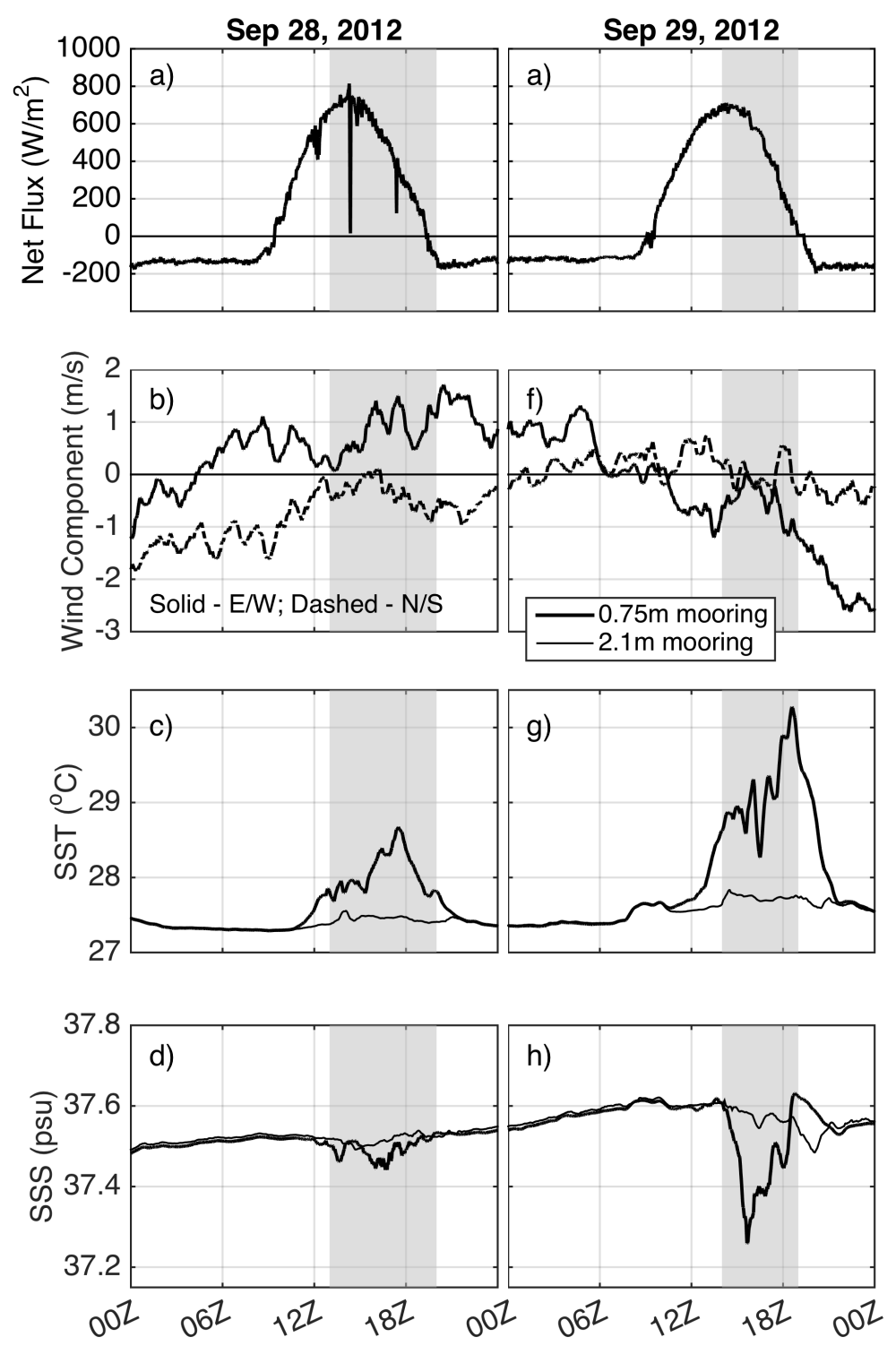

Figure 4-1: Two days of observed near-surface freshening during the SPURS-I deployment cruise on September 28 and 29, 2012: a/e) net surface heat flux; b/f) zonal and meridional wind speed; c/g) temperature observations from the mooring; $d / h$ ) salinity observations from the mooring. The grey shading indicates the time period of the advective event. 

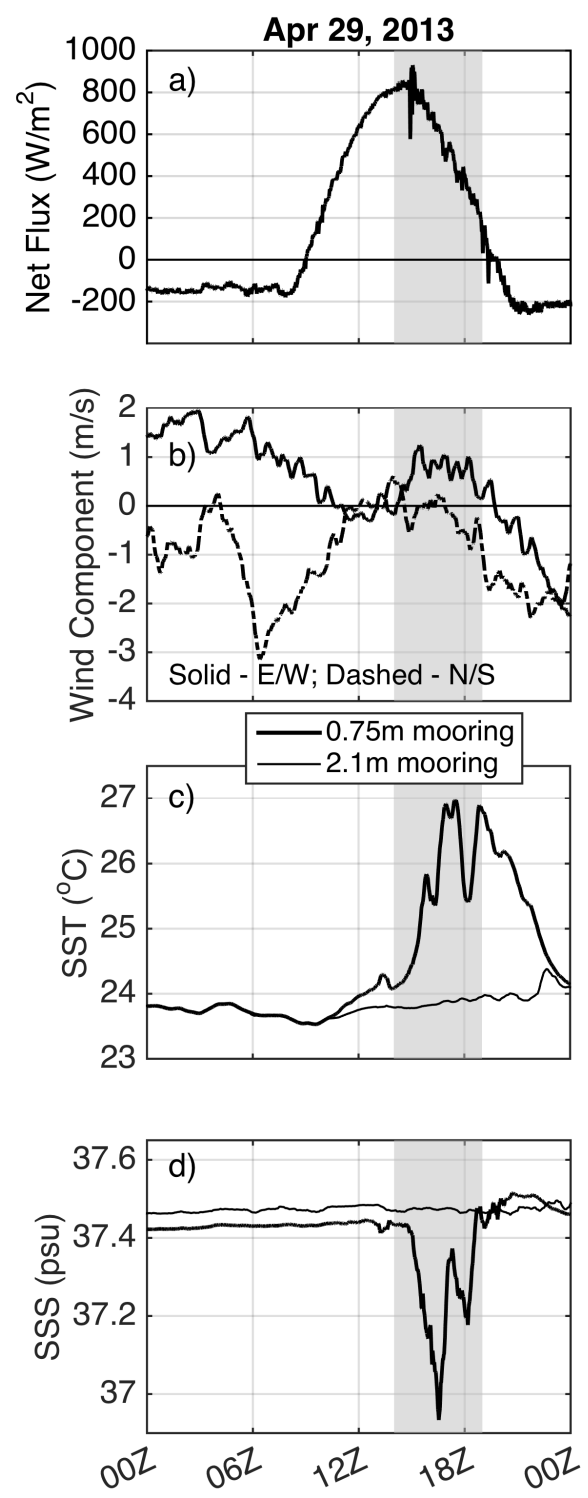

Figure 4-2: Another example day of observed near-surface freshening on April 29, 2013: a) net surface heat flux; b) zonal and meridional wind speed; c) temperature observations from the mooring $d$ ) salinity observations from the mooring. The grey shading indicates the time period of the advective event. The day was not during a cruise. 
Table 4.1: Near-surface characteristics of days with observed freshening during the daytime, including the daily average wind speed, total rainfall, and diurnal change in sea surface temperature (dSST). $\Delta \mathrm{S}$ and $\Delta \mathrm{S}_{F}$ are the observed and possible change in salinity from the surface fluxes (Eq. 4.2), respectively, over a 0.75 meter surface layer from sunrise until the maximum change in salinity during the daytime (which is time $\Delta t_{\max }$ to the nearest quarter hour). $\Delta t$ is the estimated length of the advective event, rounded to the nearest quarter hour. $\mathbf{P}$ is the total required rain fall in order to explain the near-surface freshening from precipitation.

\begin{tabular}{c|cccccc|cc} 
Date & $\begin{array}{c}\text { Wind Speed } \\
\mathrm{m} / \mathrm{s}\end{array}$ & $\begin{array}{c}\text { Rain Fall } \\
\mathrm{mm}\end{array}$ & $\begin{array}{c}\mathrm{dSST} \\
{ }^{\circ} \mathrm{C}\end{array}$ & $\begin{array}{c}\Delta \mathrm{S} \\
\mathrm{psu}\end{array}$ & $\begin{array}{c}\Delta t_{\text {max }} \\
\mathrm{hrs}\end{array}$ & $\begin{array}{c}\Delta t \\
\mathrm{hrs}\end{array}$ & $\begin{array}{c}\Delta \mathrm{S}_{\text {flux }} \\
\mathrm{psu}\end{array}$ & $\begin{array}{c}\mathbf{P} \\
\mathrm{mm}\end{array}$ \\
\hline $09 / 28 / 2012$ & 1.04 & 0.00 & 1.58 & -0.08 & 2.25 & 6.75 & 0.04 & 1.7 \\
$09 / 29 / 2012$ & 0.80 & 0.00 & 3.04 & -0.35 & 1.75 & 5.0 & 0.04 & 7.5 \\
$04 / 29 / 2013$ & 1.08 & 0.00 & 3.81 & -0.48 & 2.0 & 5.25 & 0.05 & 10.3
\end{tabular}

\subsubsection{Surface Forcing}

To gauge the possibility of rainfall as a source of the freshening, it is first useful to quantify the necessary rainfall to create the observed salinity depression. Using a virtual salt flux (Phillips, 1977; Price et al., 1986; Large et al., 1994), the change in salinity due to surface fluxes, $\Delta S_{\text {flux }}$, over a layer of depth, h, can be estimated by

$$
\Delta S_{\text {flux }}=\frac{S_{0} F_{S}}{h}
$$

where $S_{0}$ is the surface salinity, and $F_{S}$ is the total evaporation $(\mathbf{E})$ minus precipitation (P) over some time period. Conversely, the necessary total precipitation or evaporation (or combination thereof) to generate a salinity change of $\Delta S$ over a layer of depth, $h$, can be estimated by

$$
F_{S}=\mathbf{E}-\mathbf{P}=\frac{\Delta S h}{S_{0}}
$$

The magnitude of evaporation or precipitation necessary to cause an observed salinity change can be estimated. Here, the depth of the shallowest CTD is used for $h$, which is $0.75 \mathrm{~m}$.

Since no precipitation was recorded on any of the three days, the salinity increase caused by evaporation can be estimated by Eq. 4.2. Between sunrise and time of maximum observed salinity difference, the fluxes on all three days should cause between a 0.04 and 0.05 psu increase, not decrease, in salinity (Table 4.1, second to last column). The amount of precipitation necessary to explain the observed salinity depression $(\Delta S)$ is the last column 
of Table 4.1 calculated from Eq. 4.3. The observed depression on September 28, September 29, and April 29 require 1.7, 7.5, and $10.3 \mathrm{~mm}$ of precipitation, respectively.

Since the mooring only measures precipitation at a single location, two satellite precipitation products are used to explore the larger SPURS-I region. Examining the $2.5^{\circ} \mathrm{x} 2.5^{\circ}$ box of GPCP and $1^{\circ} \times 1^{\circ}$ box of CMORPH in which the mooring is located for each day (see Data section for product details), CMORPH shows no precipitation on any of the three days, and GPCP shows $0.19 \mathrm{~mm}$ of rain for the two days in September and none for the day in April. Examining within a few days of the observed freshening events, the satellite products do indicate rainfall in the region for all three days, with a significant event on September 25-27, 2012 at the mooring (created over a 1 psu change near the surface). The fresher water evidently came from a precipitation event, but the rainfall did not fall at the mooring on the days with the observed near-surface freshening.

\subsubsection{Near-surface advection}

If local (in time and space) precipitation is not the cause of the near-surface freshening, then it is likely advection. Two types of advection can cause the strong vertical salinity gradient observed near the surface (on the order of 1-10 meters in thickness): transport of an existing vertical gradient, or differential near-surface advection of a fresher water mass (Fig. 4-3). Either process could be responsible for the observed vertical structure. Without near-surface currents or near-mooring gradients, it is impossible to distinguish between the two possibilities. The vertical salinity difference between the $0.3 \mathrm{~m}$ and $6 \mathrm{~m}$ salinity record from the Wave Gliders for the days in question are generally smaller than the observed freshening (Fig. 4-4). On the two days with the largest magnitude of freshening (September 29 and April 29, Fig. 4-4b and c), the vertical salinity difference needed to explain the freshening would have to be larger than all of the observed differences on those days.

Regardless, vertical shear near the surface due to DWL current is expected since all three days are associated with diurnal warming greater than a degree (Fig. 4-1c,g, Fig. 42c, Table 4.1). Additionally, the near-surface freshening creates an even more stable layer during the daytime and may confine the trapped momentum to an even smaller layer. The salinity at the freshest point of the advective event is observed in the region by the Wave Gliders and Slocum gliders in the SPURS-I study area for both September events (Figs. 41d,h and 4-5a,b; for September 29 event in Fig. 4-5a the nearest low salinity patch that 


\section{Near surface advection of lateral gradient}

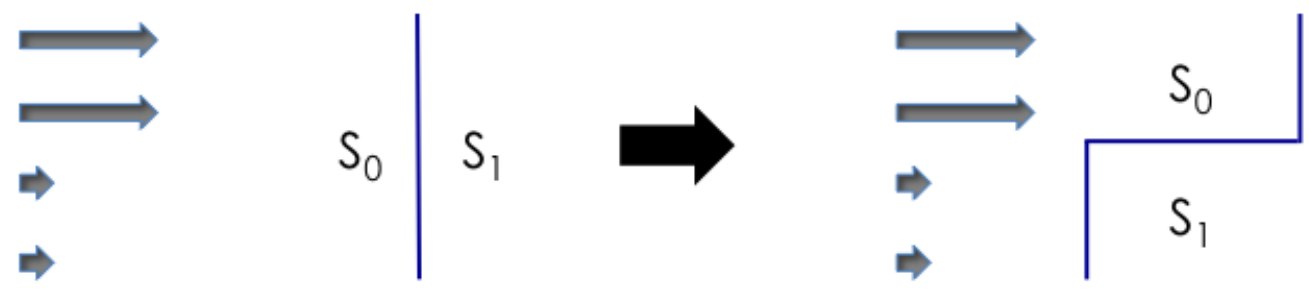

\section{Mean advection of strong vertical gradient}
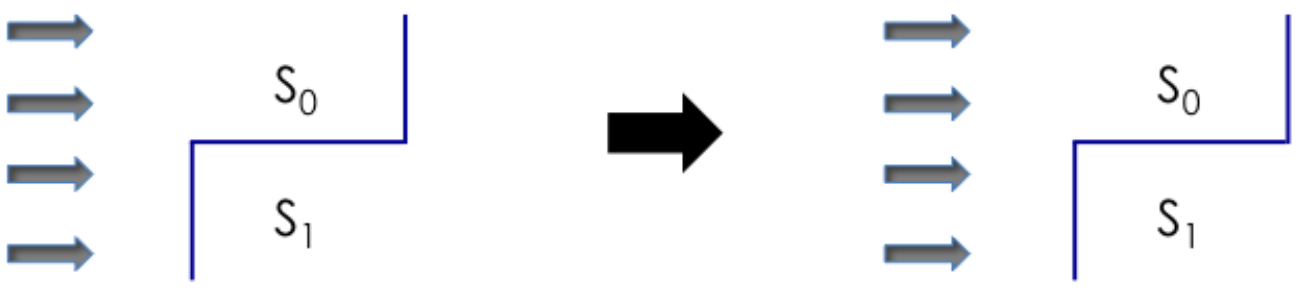

Figure 4-3: Two types of advection that can cause the observed near-surface freshening during the daytime. Top: Near-surface advection of a lateral gradient. This would be the case with a diurnal warm layer current. Bottom: Mean advection of a strong vertical gradient. 

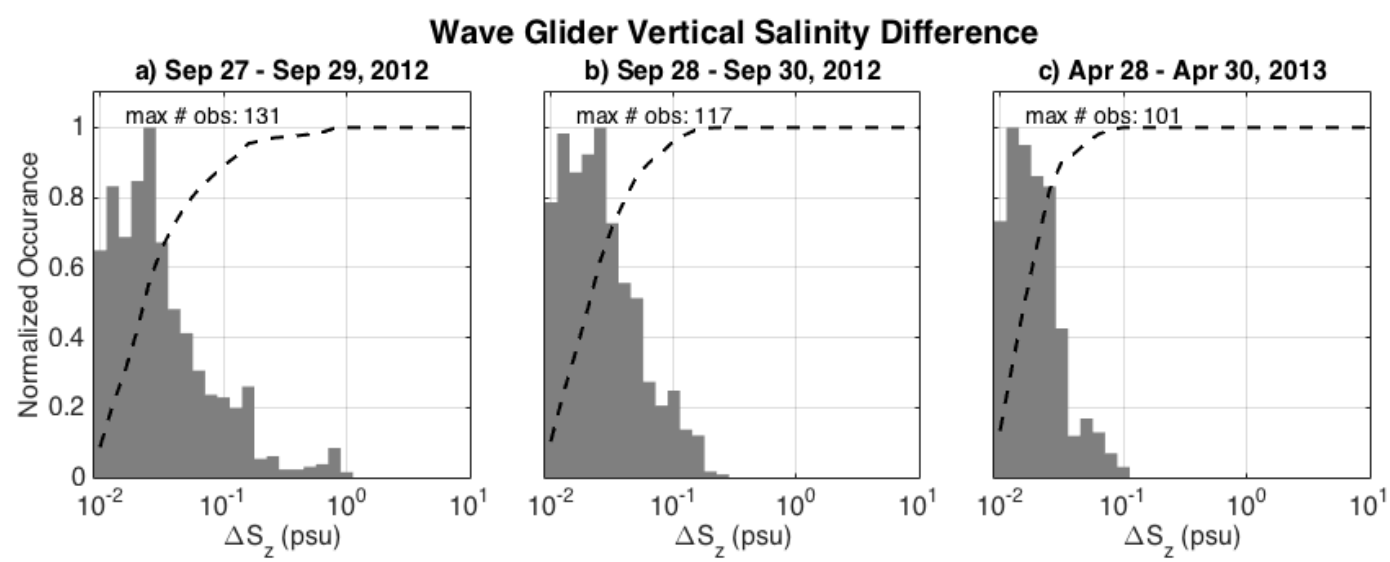

Figure 4-4: Normalized histogram of observed salinity difference between the $0.3 \mathrm{~m}$ and 6.0 $\mathrm{m}$ CTD $\left(\Delta S_{z}\right)$ of the Wave Gliders over \pm 1 day for a) September 28, 2012, b) September 29, 2012, and c) April 29, 2013. The black dotted line is the empirical cumulative distribution function. The normalization factor is included as the maximum number of observations in a bin. The histogram is cut off at $10^{-2} \mathrm{psu}$ as that is a conservative estimate of the accuracy of the CTDs (the first bin includes all vertical differences less than $10^{-2} \mathrm{psu}$ ).

explains the surface freshening at the mooring is just to the right of the " $\mathrm{X}$ " that denotes the mooring location), but not the April 29 event. The observed salinity signals of the Wave Gliders may be aliased, as any moving platform observes both spatial and temporal variability simultaneously. The salient detail is that the low salinities do exist in the region for the September events.

During the September events, Slocum glider observations and the Shipboard TSG provide better spatial coverage compared to April, and clearly show low salinity patches. There are fewer observations for the April 29 event. The closest Wave Glider is just over $20 \mathrm{~km}$ away during the 3 days around the April freshening event (Fig. 4-5c). However, the lowest salinity of the freshening event is observed in the region by the Wave Glider two days after April 29. Therefore, it is possible that the observed freshening is due to advection of gradients that are not spatially resolved during the time period in question.

\subsubsection{One-Dimensional Modeling}

Since near-surface current observations are not available, two one-dimensional mixed layer models are used to estimate the currents: the Kantha-Clayson model (Kantha and Clayson, 1994, 2004, hereafter KC) and Price-Weller-Pinkel model (Price et al., 1986, hereafter PWP). Both models are forced with the atmospheric data from the SPURS-I central mooring and 

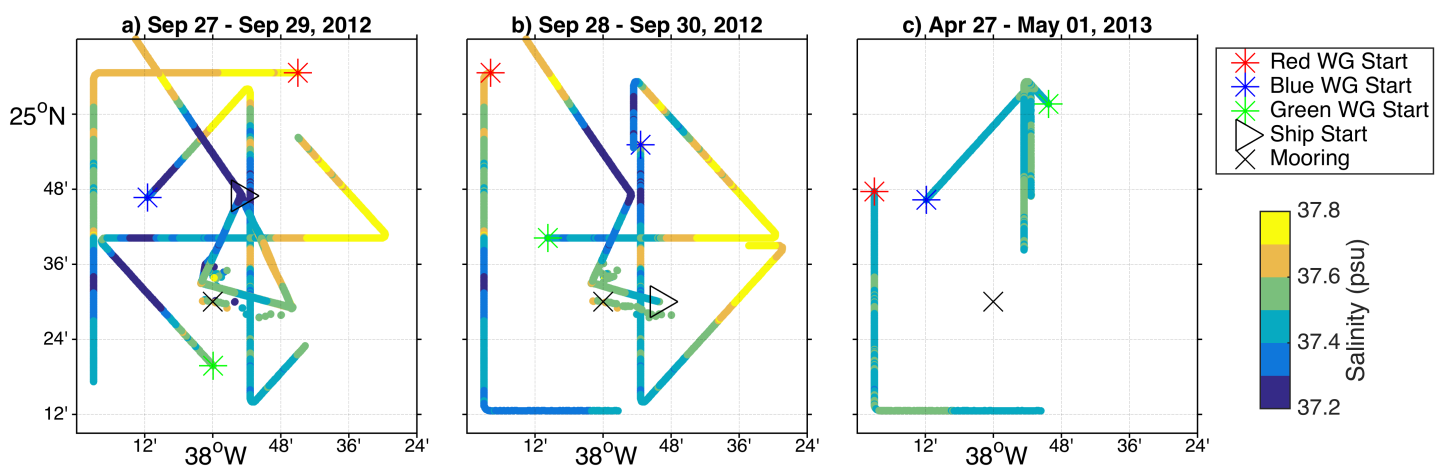

Figure 4-5: Observations of salinity from the SPURS-I study area $\left(1^{\circ} \times 1^{\circ}\right.$ box $)$ during \pm 1 day from the observed near-surface freshening on a) September 28, 2012, b) September 29, 2012 (two days shown in Fig. 4-1), and c) April 29, 2013 (day shown in Fig. 4-2) from the Slocum gliders (0.5-1 m depth, seemingly randomly dispersed dots), Wave Gliders (0.35 m), and thermosalinograph from the ship (only in a and b, nominally $5 \mathrm{~m}$ ). The starting point for a given time period of the path of each Wave Glider is denoted by a colored star and ship denoted by the triangle. The black X is the location of the SPURS-I mooring.

initialized with the observed temperature and salinity profile from the mooring. $\mathrm{KC}$ is a second-order Mellor-Yamada (Mellor and Yamada, 1974) turbulence closure model and PWP is a dynamical instability model that utilizes a Richardson number-based mixing scheme. Both models are used in Chapter 2, and the models well-reproduce the observed currents at $3.0 \mathrm{~m}$.

Both models are run with $0.1 \mathrm{~m}$ vertical resolution, and a 9-band solar extinction parameterization (Paulson and Simpson, 1981) to best reproduce the temperature profile observations. Temperature profiles are shown for each run (Fig. 4-6a-f), as well as observed profiles (Fig. 4-6g-i) every 2 hours. Rather than comparing time series at specific depths, profiles are shown. Relatively small uncertainties in the actual depth of the near-surface measurements can lead to quite different observations under strong stratification. Salinity profiles are not shown. The models do not reproduce the freshening, and are not expected to reproduce advection. Both models reproduce a diurnal cycle of temperature and reproduce the September 28 warming (Fig. 4-6a,d). However, KC underestimates the warming on the higher diurnal warming days (September 29 and April 29, Fig. 4-6b,c), likely due to larger than realistic background mixing levels as noted by Bogdanoff et al. (In Preparation). PWP does reproduce the magnitude of the warming for the higher diurnal warming days (Fig. 4-6e,f). The vertical distribution of density, and therefore temperature, will change 
the vertical distribution of momentum in the upper ocean.

The models have quite different DWL shear, as expected with the varying temperature profiles. Changes in near-surface stratification will change the near-surface current structure. Without near-surface observations with which to compare, the models only provide a plausible range of currents in the near-surface ocean. Profiles of current speed for both models are provided in Fig. 4-7, for the model runs initialized with no current. The initial current selection did not change the total shear $\left(\sqrt{u_{z}^{2}+v_{z}^{2}}\right)$ in the near-surface, but did change the directional shear (change in current direction with depth) and speed shear (change in current speed) magnitudes.

There are similarities in the model prediction of currents, such as general agreement of the time of maximum or minimum current speed. The models do reproduce a DWL current for each of the days. On September 28 (Fig. 4-7a,d), the winds shift direction causing the DWL current to manifest as a reduction in the near-surface current. Thus, although the current has a smaller magnitude near the surface than it does deeper in the ocean, there is still significant shear in the upper ocean. On September 29 (Fig. 4-7b,e) and April 29 (Fig. 4$7 \mathrm{c}, \mathrm{f})$ the near-surface current is accelerated during the daytime. The models on those days reproduce a DWL current of over $0.1 \mathrm{~m} / \mathrm{s}$. Differences between the vertical distribution of momentum input from the wind by PWP and $\mathrm{KC}$, account for some of the disparity between the modeled near-surface currents.

For these particular days, the PWP scaling (Price et al., 1986) of the "diurnal jet" estimates a peak $0.1-0.15 \mathrm{~m} / \mathrm{s}$ jet for each of the days in question. Kudryavtsev and Soloviev (1990) found a fairly constant speed of the DWL regardless of wind speed associated with diurnal warming meaning a DWL current is expected regardless of the strength of the warming. However, their observations were during periods of higher wind speeds (about $5 \mathrm{~m} / \mathrm{s}$ ) in which strong diurnal warming is not expected. Additionally, Price et al. (1986) and Fairall et al. (1996) found no obvious dependence of the DWL current speed on wind stress. With only 3 days of modeled currents, no significant conclusions can be made about near-surface currents. As such, for much of the remainder of this study, a DWL current of $0.1 \mathrm{~m} / \mathrm{s}$ is assumed however, variable speeds are investigated where appropriate (this is a current on top of any background current, such as the geostrophic current). 

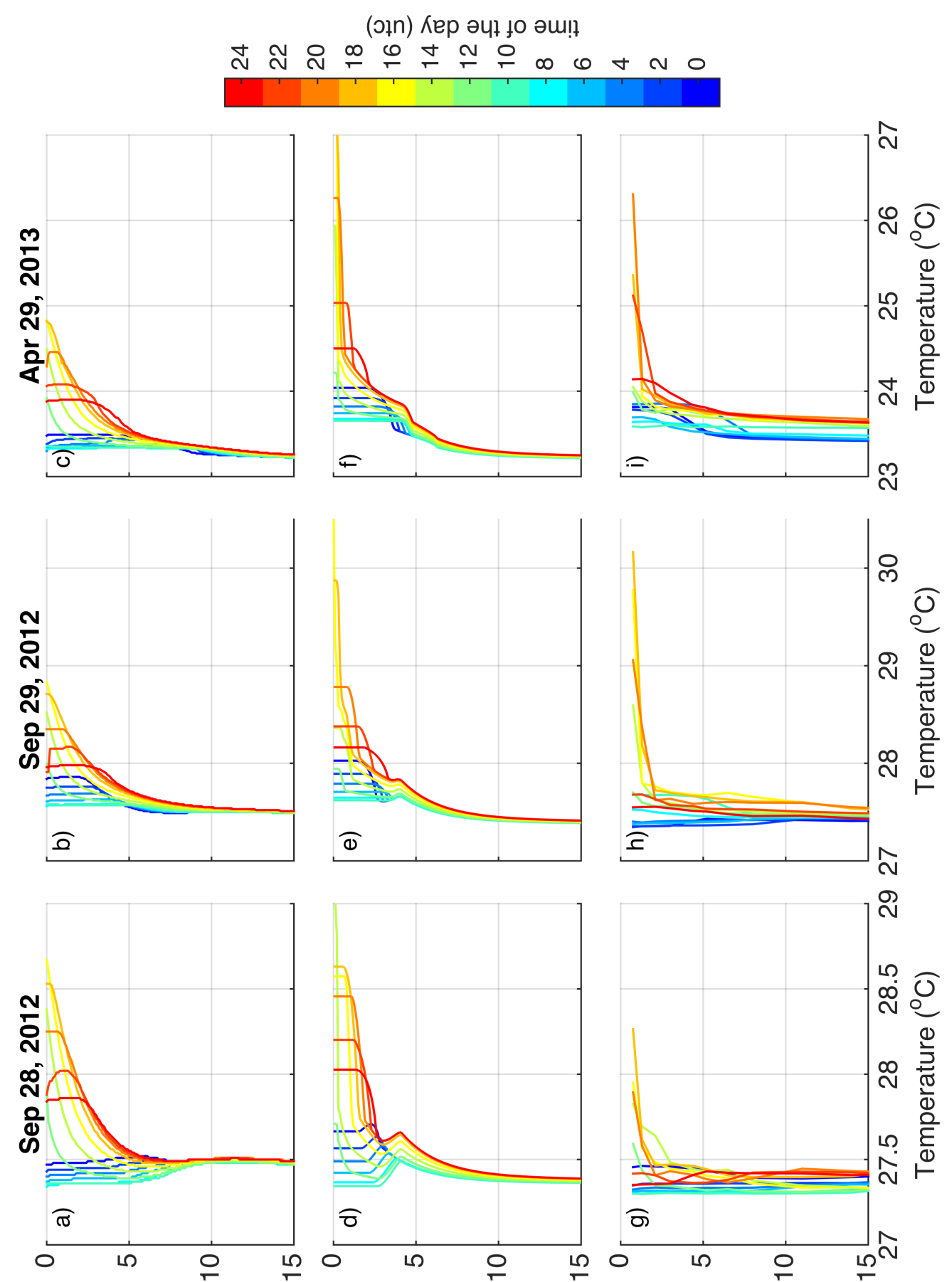

(i) 4ұdəp

(u) чldəp uoske|O-еціuеप्र

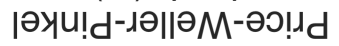

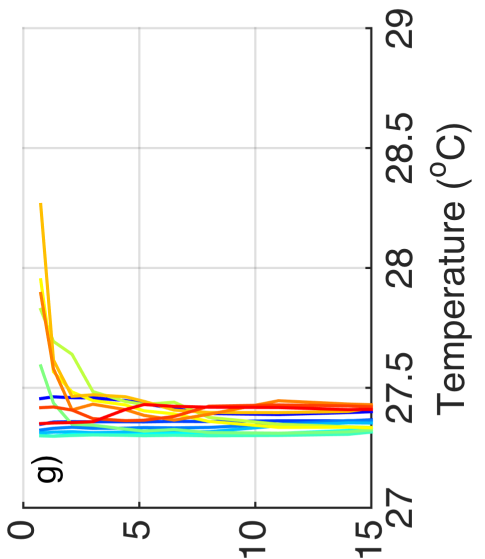

(u) чldəp suo!̣enısso

Figure 4-6: Modeled and observed temperature profiles every 2 hours for the three days with observed near-surface freshening, September 28 and 29, 2012 and April 28, 2013 from a/b/c) Kantha-Clayson, d/e/f) Price-Weller-Pinkel, and g/h/i) the SPURS-I central mooring. The color indicates the time of the day. Note the different temperature scale for each day. 

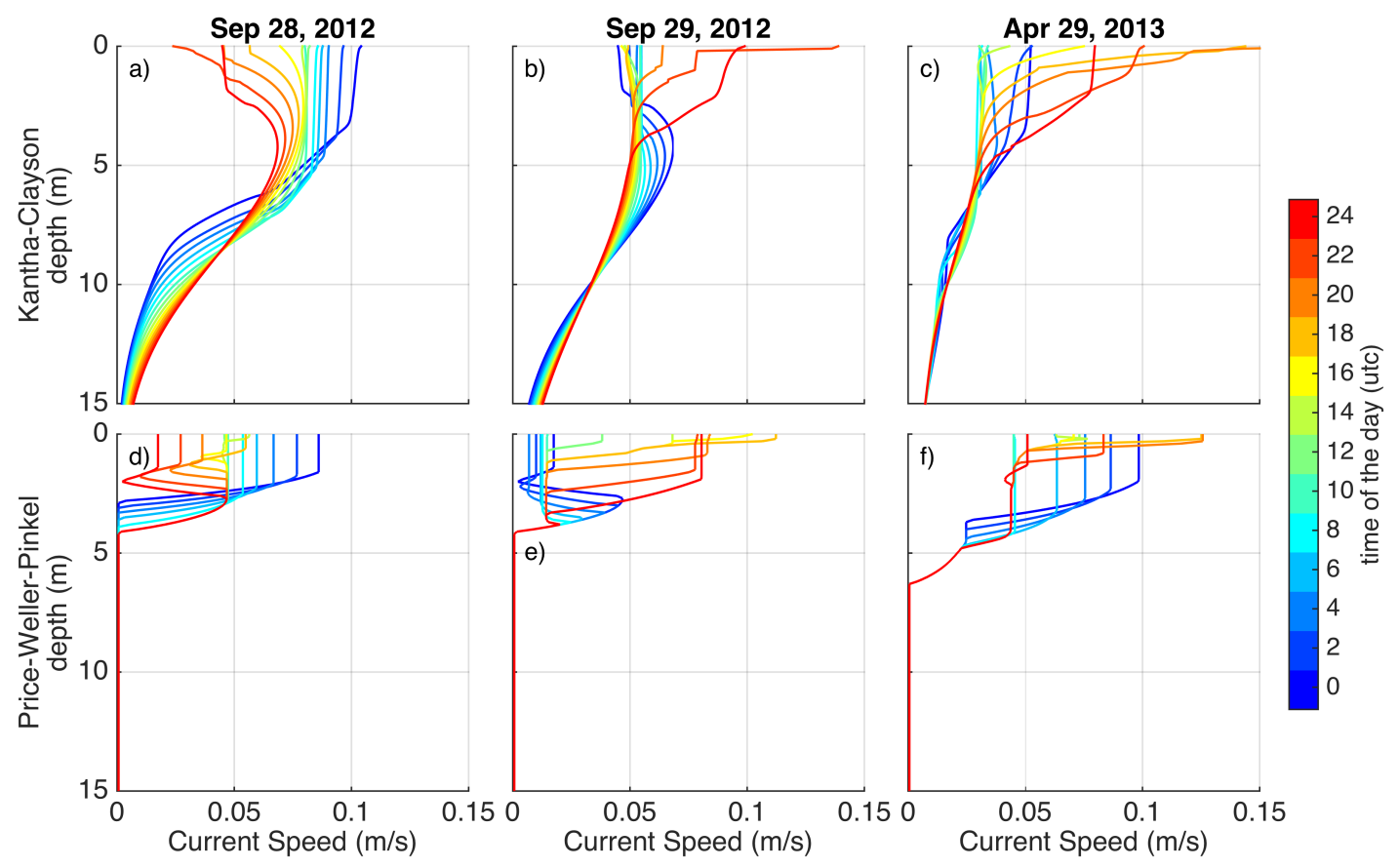

Figure 4-7: Modeled current speed profiles every 2 hours for the three days with observed near-surface freshening, September 28 and 29, 2012 and April 28, 2013 from a/b/c) KanthaClayson and d/e/f) Price-Weller-Pinkel models. The color indicates the time of the day. 


\subsubsection{Motivation of the mixing mechanism}

There are other examples of days with salinity changes greater than expected by the surface fluxes, but the focus of this study is on days in which the changes cannot be explained by surface fluxes or vertical mixing. It is likely a confluence of events that allows us to observe the near-surface advection. Strong diurnal warming confines the advection to the near-surface, and a strong horizontal salinity gradient happened to be present near the mooring. However, this near-surface advection associated with the DWL current is likely present whenever diurnal warming occurs, just over varied depths depending on the depth of the DWL.

We have determined that local precipitation is not the cause of the near-surface freshening, but does create gradients in salinity that can last several days. Then, shear associated with the diurnal warm layer, which occurs over a fractional depth of the mixed layer, horizontally displaces water near the surface during the daytime converting horizontal gradients into vertical ones. In the case of September 28 the shear causes the DWL to move less far than the deeper mixed layer water. At night, convection vertically mixes the horizontally displaced water throughout the (deeper) mixed layer, creating a well-mixed upper ocean. This cycle of advection and then vertical mixing, in effect, laterally mixes horizontal inhomogeneities in the mixed layer.

\subsection{Simple model of mixing}

Consider the case of a pre-existing horizontal gradient of salinity in the mixed layer and vertical shear across the mixed layer due to the DWL current during the daytime coupled with nocturnal convection. We consider a simple representation of the cycle of advection then vertical mixing with the winds in the same direction each day. A schematic diagram is shown in Fig. 4-8. In general, the DWL current has vertical shear and is not uniform (see Fig. 4-7). Here, the shear layer is considered a DWL current with a velocity $u_{0}$ uniform over the entire DWL. Price et al. (1986) and Kudryavtsev and Soloviev (1990) note that the DWL current does not vary much with wind speed or depth of the DWL. The initial salinity of the two areas are $S_{0}$ and $S_{1}$, where $S_{1}=S_{0}+\Delta S_{0}$. Given a unidirectional current in the positive $\mathrm{x}$-direction, over the course of the day, the diurnal shear layer (which has a thickness $\delta$ ) can transport water with salinity $S_{0}$ a distance $\Delta x$, given by $\Delta x=u_{0} \Delta t$, where 


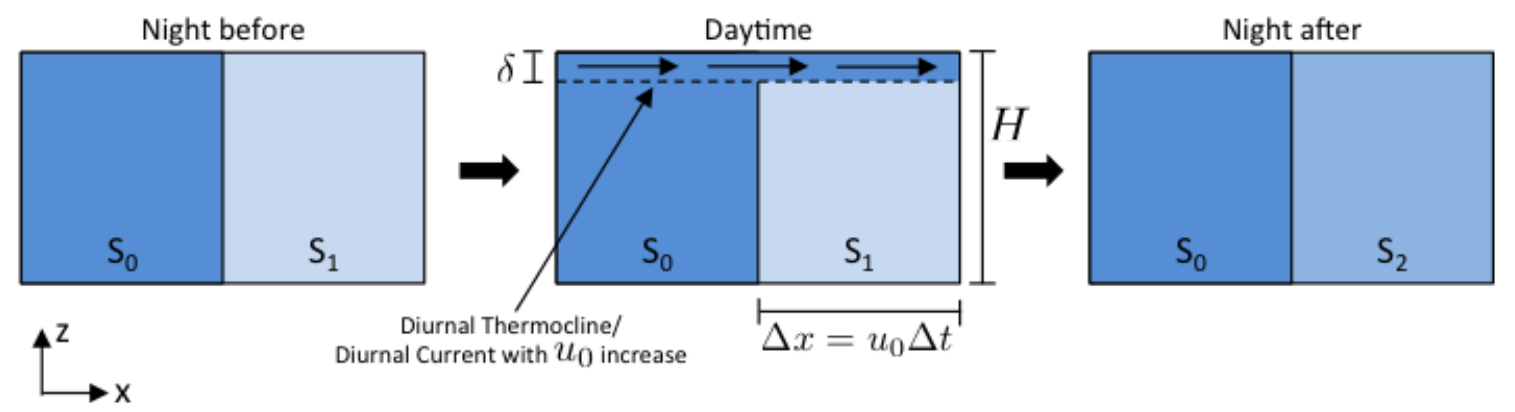

Figure 4-8: Schematic of the simplified representation of the mixing mechanism. The night before a front (delta gradient) exists over a well-mixed layer of depth, H. During the daytime diurnal warm layer shear exists at the near-surface due to stabilization from incoming solar radiation over a layer of depth, $\delta$, with a current $u_{o}$ assuming no background current of the mixed layer. The surface layer can travel a distance $\Delta x$ over a diurnal cycle that is $\Delta t$ long. Nocturnal convection ensues when the net surface flux changes sign, cooling the ocean surface. A new salinity $S_{3}$ will be observed in the region where differential advection by the diurnal warm layer shear occurred at the near-surface, which is in between the two observed salinities the night before.

$\Delta t$ is the length of time the DWL exists and the advective time-scale. At night, convection mixes the layer to a mixed layer depth, $H$.

Considering an infinite reservoir of $S_{0}$ water, this cycle will eventually laterally mix the regions to have very similar salinities. If $n$ is the number of times this cycle occurs, then the salinity of downstream (or downwind) box can be written non-dimensionally as

$$
\frac{S_{n+1}}{S_{0}}=\left(1+\frac{\Delta S_{0}}{S_{0}}\right)\left(1-\frac{\delta}{H}\right)^{n}+\frac{\delta}{H} \sum_{k=0}^{n-1}\left(1-\frac{\delta}{H}\right)^{k}
$$

where $S_{n+1}$ is the salinity of the right box after $n$ mixing cycles. This allows us to explore the system in terms of two non-dimensional parameters: the ratio of the depth of the DWL to the mixed layer depth $(\delta / H)$ and the ratio of the initial salinity difference between the two regions to the salinity of the reservoir on the upwind (against the direction of the current) side $\left(\Delta S_{0} / S_{0}\right)$.

The physical and mathematical limitations are as follows. First, $|1-\delta / H|<1$ for convergence, and physically $\delta \leq H$. When $\delta=H$, pure advection of $S_{0}$ water mass over the entire depth $H$ occurs and thus, no mixing is necessary to obtain similar salinities. Second, while negative salinities are mathematically possible, it does not represent physical reality. 
A $\Delta S_{0}<0$ would indicate a saltier rather than fresher reservoir. Thus, $\Delta S_{0} / S_{0} \geq-1$ so that the salinity on the (downwind) right side is not negative. Third, if saltier water is being advected atop fresher water, the salinity difference must be less than 2.5 times $(\beta / \alpha$, saline contraction coefficient over the thermal expansion coefficient) smaller than temperature difference across the diurnal thermocline so that the water column does not convectively overturn. Fourth, in reality, both the speed and direction of the diurnal shear layer vary over the course of a day. This is not considered. Lastly, there is realistically not an infinite reservoir of $S_{0}$ water and the salinity gradient is constantly changing. However, while it is an important assumption in the calculation of the number of cycles needed to erase a gradient, it does not affect the diffusivity calculation. The infinite gradient works for the simple model, as the estimate of effective diffusivity does not depend on the gradient (see Eq. 4.6).

The number of cycles required to bring the salinity $S_{n+1}$ to within $99.9 \%$ of $S_{0}$ is shown in Fig. 4-9. The exact number of cycles depends on the selection of the threshold. Additionally, the length of time of each cycle only determines the distance over which the advective event will occur. Here, we assume a constant $\Delta x$ and therefore, assume a constant $\Delta t$. The 99.9\% threshold is chosen such that non-dimensional salinity differences up to 0.001 (or roughly $0.035 \mathrm{psu}$ ) are considered. The September 29 case study is shown as an example, and denoted as the black asterisk on Fig. 4-9. Given a $1 \mathrm{~m}$ DWL and $20 \mathrm{~m}$ mixed layer, roughly 49 cycles would be required to sufficiently mix out the observed salinity difference. The observations do not quite fit the simple model as the salinity reduces throughout the afternoon, which may be due to advection of a horizontally inhomogeneous gradients or deepening of the DWL throughout the afternoon. While a deepening of the DWL is present in the observations and depths below $2.1 \mathrm{~m}$ do get slightly fresher throughout the afternoon on September 29 and April 29, the temporal variability in the measurements does not allow for any definite conclusions. However, small changes can substantially change the number of cycles required for mixing out the gradient. For a salinity difference 3 times smaller and a DWL one-third the depth of the mixed layer (DWL under weaker diurnal warming are deeper, and cases like these were observed during SPURS-I), only three cycles are necessary to mix out the gradient. For smaller salinity differences and larger non-dimensional depths (physically, the DWL depth is a larger fraction of the mixed layer) the number of cycle required to sufficiently reduce the salinity difference is smaller. 


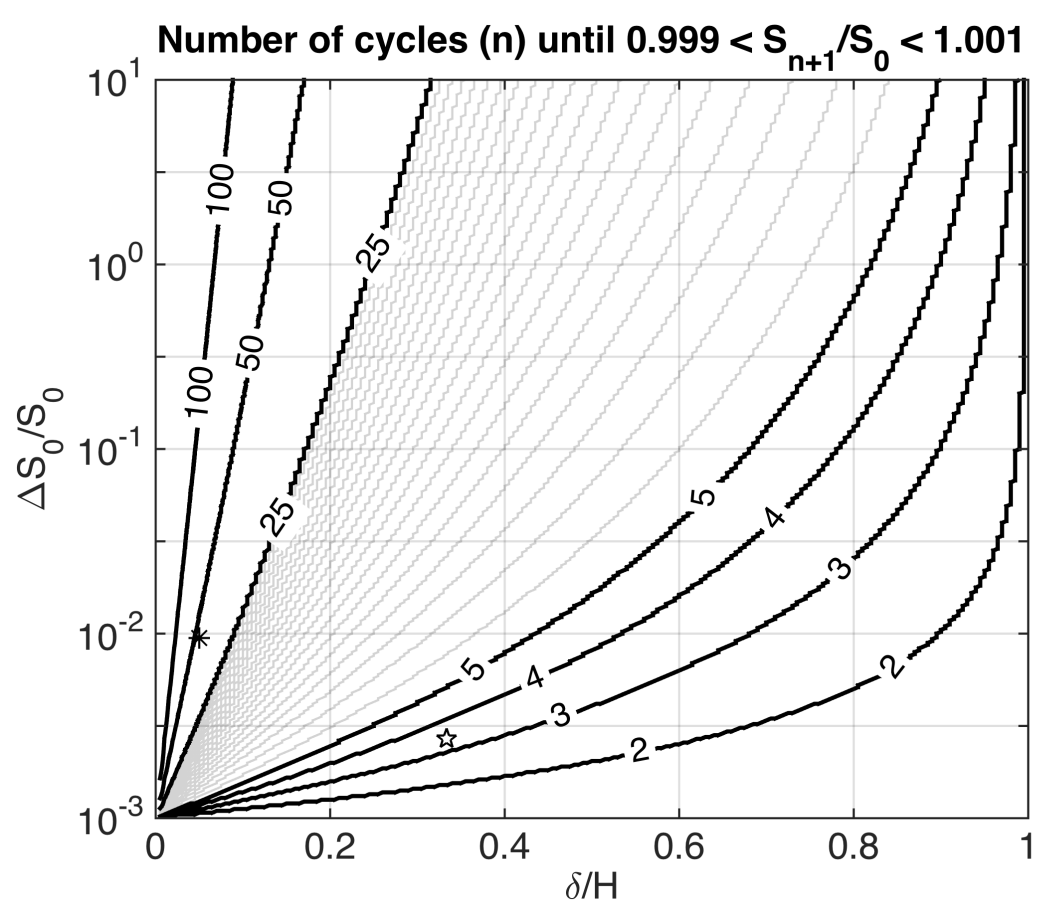

Figure 4-9: The number of times, $n$, the cycle of advection then mixing would have to occur in order for the salinity in the right box of Fig. $4-8$ to be within $99.9 \%$ of $S_{0}$. The number of cycles is plotted as a function of the non-dimensional salinity change and nondimensional depth of the diurnal warm layer depth. The black astrisk indicates the number of cycles necessary to erase the observed $\Delta S$ (corresponding so a $\Delta S_{0}$ ) on September 29, 2012 assuming a $20 \mathrm{~m}$ mixed layer and $1 \mathrm{~m}$ diurnal warm layer. The black star indicates the number of cycle necessary to erase a $0.1 \mathrm{psu}$ difference assuming a diurnal warm layer that is $1 / 3$ the depth of the mixed layer (which assumes weaker diurnal warming). 
Vertical mixing or homogenization does not change the vertically averaged salt content of a water column, so the episodic mixing can be directly related to the advection over the time interval between mixing events. The vertically-averaged (denoted by the over bar) equation for conservation of salt can be expressed as

$$
\frac{\partial \bar{S}}{\partial t}+\bar{u} \frac{\partial \bar{S}}{\partial x}=-\frac{\partial \overline{u^{\prime} S^{\prime}}}{\partial x}=\kappa_{\mathrm{eff}} \frac{\partial^{2} \bar{S}}{\partial x^{2}}
$$

where $\bar{S}$ is vertically-averaged salinity, $\bar{u}$ is the vertically-averaged zonal velocity, the primed terms denote variations from the mean, and $\kappa_{\text {eff }}$ is the effective horizontal diffusivity of the system. An effective diffusivity of the model can be calculated following Ferrari and Young (1997), hereafter FY97, which refer to this cycle of advection and then mixing as "vertical homogenization at intervals of $[\Delta t]^{\prime}$, where $\Delta t$ is the length of the interval of advection. The reference frame of the system is modified such that the vertically-averaged horizontal velocity is zero. Even with a zero net horizontal transport, an effective flux of salt (or any tracer) is associated with this cycle of advection and episodic mixing. The change in the reference frame requires advection in the opposite direction below the DWL.

The effective horizontal diffusivity (FY97, Eq. 2.9c) is estimated by calculating mean squared velocity as a function of $u_{o}$ and $\delta / H$ where

$$
\kappa_{\mathrm{eff}}=\frac{1}{2} \Delta t \overline{u^{2}}=\frac{1}{2} \Delta t u_{o}^{2} \frac{\delta}{H}\left(1+\frac{\delta}{H}\right) .
$$

Note that the magnitude of the gradient is not required to estimate the diffusivity. As shown by FY97, the advection and then vertical mixing example used here does not require a regular occurrence of the mixing mechanism. The effective diffusivity scales linearly with $\Delta t$. Therefore, the advective time-scale can vary, and the average diffusivity would be a function of the average advective time-scale. The effective horizontal diffusivity is estimated assuming a six hour advective time-scale for several different DWL currents as a function of $\delta / H$ in Fig. 4-10. The maximum diffusivity for a given $u_{0}$ occurs at $\delta / H=0.5$. Physically, when the DWL is half the depth of the mixed layer, the maximum transport in each direction occurs (each direction has equal and opposite transport), and thus maximum flux of tracer or salt occurs. The values of effective diffusivity range from $1-250 \mathrm{~m}^{2} / \mathrm{s}$, over length scales from roughly $1-8 \mathrm{~km}$ (taking $u_{0}=0.3 \mathrm{~m} / \mathrm{s}$ to be upper bound on DWL current given realistic atmospheric forcing; Soloviev and Lukas, 2006). The flux of salt (or any tracer) is the 


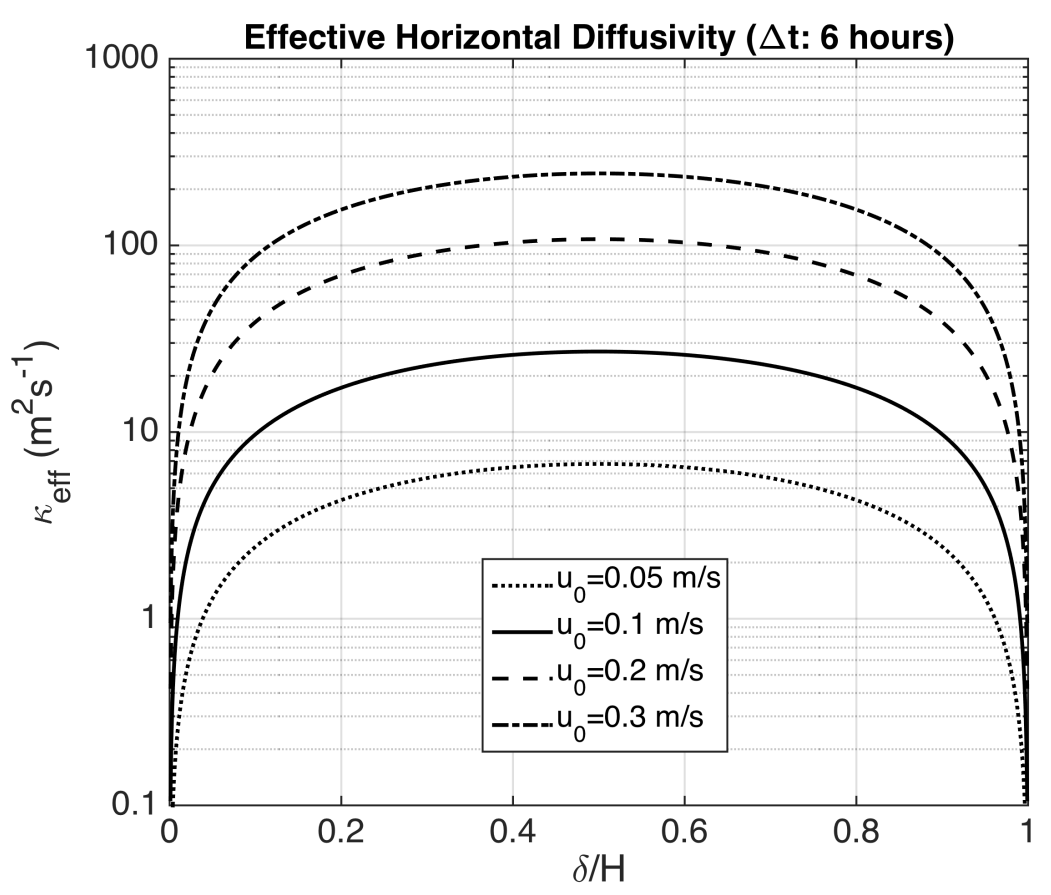

Figure 4-10: Estimated effective horizontal diffusivity from the simple model for a range of diurnal warm layer velocity increases $\left(u_{0}\right)$, for an advective time-scale of 6 hours using Eq. 4.6.

effective diffusivity times the gradient on which the diffusion acts. The relative importance of the scale of the diffusivity and observed gradients are investigated in the next section.

While this simple model provides physical insight, there are several limitations. By selecting $\bar{u}=0$, the vertically-averaged advection is zero. This may not be the case with the DWL shear and a net advection over longer time periods is obviously an important source of transport in the mixed layer. Furthermore, vertical turbulent diffusion during the advective period is not explicitly taken into account and may be an important source of mixing, especially with strong gradients. Lastly, the DWL current is assumed to be vertically homogenous. A more realistic case may be a mix of this intermittent mixing mechanism combined with a Taylor shear dispersion (Taylor, 1953) as the DWL current is sheared over the depth of the DWL.

The cases observed here have relatively small DWL depths compared to the depth of the mixed layer at night. Relatively infrequent strong diurnal warm days will tend to have small $\delta / H$. Weaker diurnal warming days, which occur more frequently (see Fig. 4-11) tend to have a deeper DWL. Given the same mixed layer depth for strong and weaker diurnal 


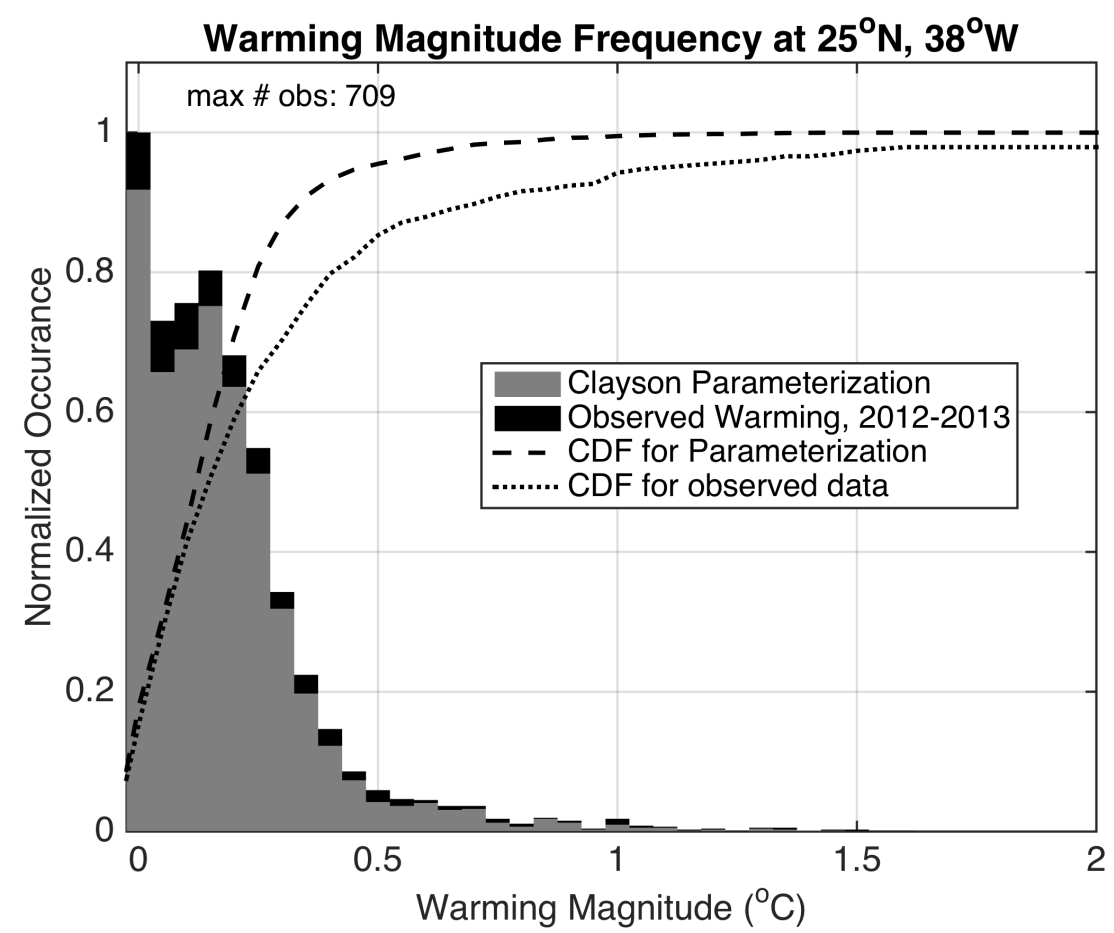

Figure 4-11: Normalized stacked histogram of diurnal sea surface temperature (dSST, maximum during the daytime minus minimum at night) from the Clayson diurnal warming parameterization of Bogdanoff et al. (In Preparation) over the 10 year periods from January 1, 1998 - December 31, 2007 (grey) and observed dSST from the over the 383 day long deployment of SPURS mooring (black). The black dotted lines are empirical cumulative distribution function. The normalization factor is included as the maximum number of observations in a bin.

warming, the weaker diurnal warming day will have a greater effective horizontal diffusion in the mixed layer. This concept relies on the idea that the speed of the DWL current does not vary much with wind speed, as noted by Price et al. (1986) and Kudryavtsev and Soloviev (1990). This is explained by the thickness of the DWL increasing at about the same rate as the momentum input increase. However, more detailed observations of the current of the upper ocean are necessary to put better limitations on the speed of the DWL current, and the vertical structure of shear in the near-surface ocean. 


\subsection{Estimate of mixing and advection}

\subsubsection{Estimate of effective diffusivity for observed cases}

Using the simple proposed model for effective mixing by the DWL current, effective diffusivities can be estimated for the three case study days using Eq. 4.6. For a $20 \mathrm{~m}$ mixed layer and 1 meter DWL using $\Delta t$ from Table 4.1 and $u_{0}=0.1 \mathrm{~m} / \mathrm{s}, \kappa_{\text {eff }}$ for September 28 is 6 $\mathrm{m}^{2} / \mathrm{s}$, September 29 is $5 \mathrm{~m}^{2} / \mathrm{s}$, and April 29 is $5 \mathrm{~m}^{2} / \mathrm{s}$. There is a squared relationship with $u_{o}$ such that a DWL current half as fast will result in a diffusivity four times smaller. Using the model current results (Fig. 4-7), which range from 0.05 to $0.15 \mathrm{~m} / \mathrm{s}$, instead of assuming $0.1 \mathrm{~m} / \mathrm{s}$, the diffusivities range from $1-10 \mathrm{~m}^{2} / \mathrm{s}$. The actual advective time-scale is likely longer than just the time of the observed near-surface freshening because DWL shear can exist during the entire period in which the net heat flux is stabilizing the ocean and even once the heat flux goes negative until the layer is mixed out by convection or wind shear. In addition, the DWL shear likely occurs over a deeper layer than $1 \mathrm{~m}$. Thus, these numbers may be an underestimate of the effective diffusivity.

The effective diffusivities found here are comparable to previous studies. Okubo (1971) found mixed layer diffusivities of roughly $1 \mathrm{~m}^{2} / \mathrm{s}$ for $1 \mathrm{~km}$ scales based on data from dye releases. Poje et al. (2014) found significant energy at currents examining scales less than $10 \mathrm{~km}$, with a $1 \mathrm{~km}$ diffusivity of roughly $30 \mathrm{~m}^{2} / \mathrm{s}$. Dye releases associated with the Scalable Lateral Mixing and Coherent Turbulence (LatMix) experiment indicate upper ocean diffusivities of $1 \mathrm{~m}^{2} / \mathrm{s}$ at a $1-5 \mathrm{~km}$ scale however, the dye was release below the mixed layer (Shcherbina et al., 2015; Sundermeyer et al., In Preparation). Diffusivity has a scale relationship, and diffusivities on 1-10 km scale are expected to be smaller than mesoscale eddy diffusivities (Callies and Ferrari, 2013).

Abernathey and Marshall (2013) estimate eddy diffusivity on spatial scales of roughly $200 \mathrm{~km}$ or greater, and the SPURS-I region in the subtropical North Atlantic gyre is a region of reduced mesoscale activity (estimated to be $0-100 \mathrm{~m}^{2} / \mathrm{s}$ ). The magnitudes of the effective diffusion associated with DWL shear in the SPURS-I region may be the same order of magnitude as, or just an order of magnitude smaller than the eddy diffusivities, regardless of scale dependence. Additionally, this region has a relatively high percentage of days with diurnal warming, during which this mechanism is expected to occur. Given the intermittent nature of diurnal warming however, the magnitude of the average diffusivity of a region over 
a longer time-scale will be proportional to the mean advective time-scale and the percentage of days with diurnal warming. Fig. 4-11 is a histogram of parameterized diurnal warming (maximum during the daytime minus minimum at night, parameterized based on wind speed, solar insolation, and precipitation) from (Bogdanoff et al., In Preparation, referred to as the Clayson parameterization) for January 1, 1998 - December 31, 2007. Included are the observed diurnal warming magnitude (or dSST for diurnal sea surface temperature) from $0.75 \mathrm{~m}$ CTD of SPURS-I central mooring. While only $6 \%$ of the days in the region have diurnal warming greater than $0.5^{\circ} \mathrm{C}$, over $80 \%$ of the days have warming greater than $0.1^{\circ} \mathrm{C}$. It is likely that some amounts of DWL shear exists with weak diurnal warming, since even relatively weak stratification can trap momentum from the wind near the surface and cause horizontal diffusion by this shear dispersion mechanism.

\subsubsection{Estimate of advection}

In order to estimate the near-surface advection by the DWL shear and subsequent effective lateral mixing by nocturnal convection, an estimate of the near-surface current, temperature and salinity gradients, DWL depth, and mixed layer depth are necessary. Current observations from the mooring are hourly and limited to below 3.5 meters and not coincident with gradient observations. Chapter 2 shows a clear enhancement of near-surface current during the daytime under DWL conditions. The shipboard TSG (when available) and Wave Gliders provide estimates of salinity and temperature gradients near the surface in the SPURS-I study area. Using the mooring temperature record and turbulence data from the Slocum gliders during the SPURS-I deployment cruise, a mixed layer depth is estimated. A depth of $20 \mathrm{~m}$ is estimated and used for all three days. A DWL depth of $1 \mathrm{~m}$ is assumed for the three case studies. However, the model results (Fig.4-7) show that the layer thickness of the DWL, and thus DWL current may greater than $1 \mathrm{~m}$. These estimates are likely conservative.

The change in concentration of a tracer, $C$, due to advection is directly related to the current, $u$, and the gradient on which the current is acting. This is shown mathematically as

$$
\frac{\partial C}{\partial t}=-\vec{u} \cdot \nabla \vec{C}
$$

where the tracer in question is salinity. Therefore, for an estimated change in salinity $(\Delta S)$ over the time to the maximum change $(\Delta t)$ shown in Table 4.1, and assumed DWL current, 

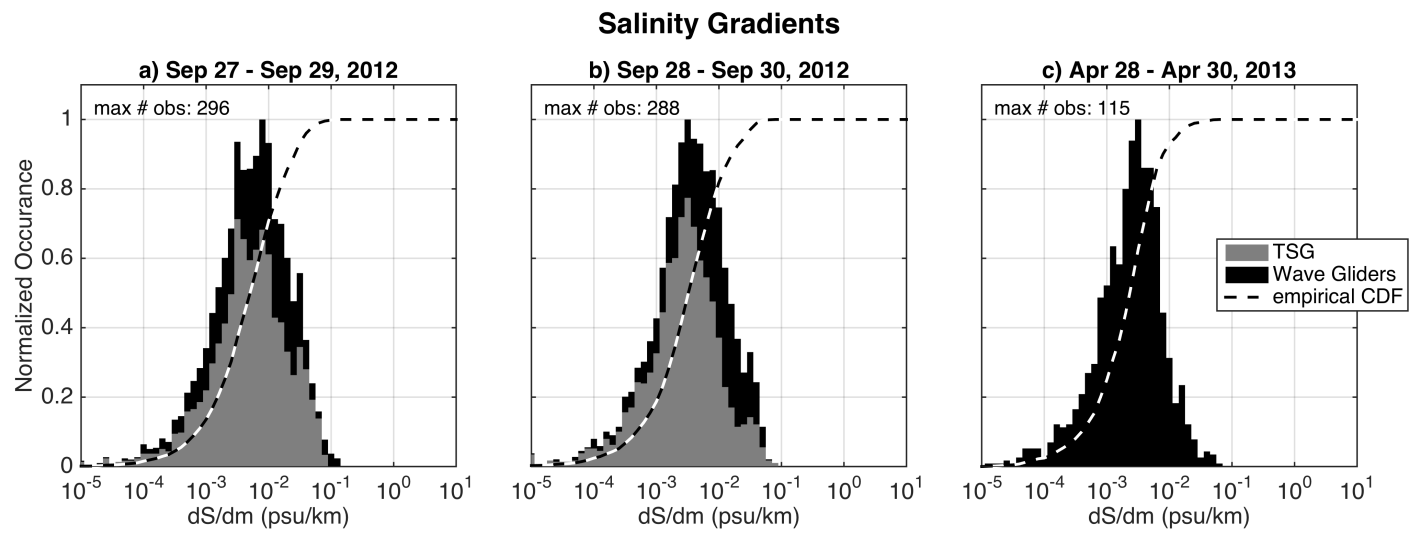

Figure 4-12: Normalized stacked histogram of observed lateral salinity gradients over \pm 1 day for a) September 28, 2012, b) September 29, 2012, and c) April 29, 2013 from the shipboard thermosalinograph (when available, black, nominally at $5 \mathrm{~m}$ ) and Wave Gliders (grey, $0.35 \mathrm{~m}$ ). The black dotted line is the empirical cumulative distribution function. The gradients are calculated in the direction of the instrument movement, $m$. The normalization factor is included as the maximum number of observations in a bin.

the necessary salinity gradient can be calculated. Gradients within a few kilometers of the mooring are rarely available, and statistics of the gradients observed in the SPURS-I region are used instead (Figs. 4-12 and 4-13). The gradients are smoothed ( $1 \mathrm{~km}$ ) to remove high frequency variability however, some of the large temperature and salinity gradients may still be due to internal waves. The direction of the gradient is computed in the direction of the instrument motion. Since the direction of the current will change the magnitude and sign of advection, the magnitude of the gradient is the important factor. The observed gradients from the Wave Gliders and TSG \pm 1 day around the day with observed freshening are used.

Larger salinity gradients are present for September 28 likely due to the large rain event that occurred a few days prior associated with tropical system Nadine (2012 Atlantic Hurricane Season). Given a $0.1 \mathrm{~m} / \mathrm{s}$ DWL current in the direction of the salinity gradient, the gradients necessary for the observed freshening are $0.01 \mathrm{psu} / \mathrm{km}$ (largest $99 \%$ of observed gradients during \pm 1 day around September 28), $0.56 \mathrm{psu} / \mathrm{km}$ (100\%, the largest observed gradients during \pm 1 day around September 29), and $0.67 \mathrm{psu} / \mathrm{km}(100 \%)$, for September 28, September 29 and April 29, respectively. The large percentages indicate that the required salinity gradient for advection to explain the freshening occur infrequently, but do exist. In addition, relatively small low-salinity patches likely exist in the ocean that were not observed by the TSG or Wave Gliders. 

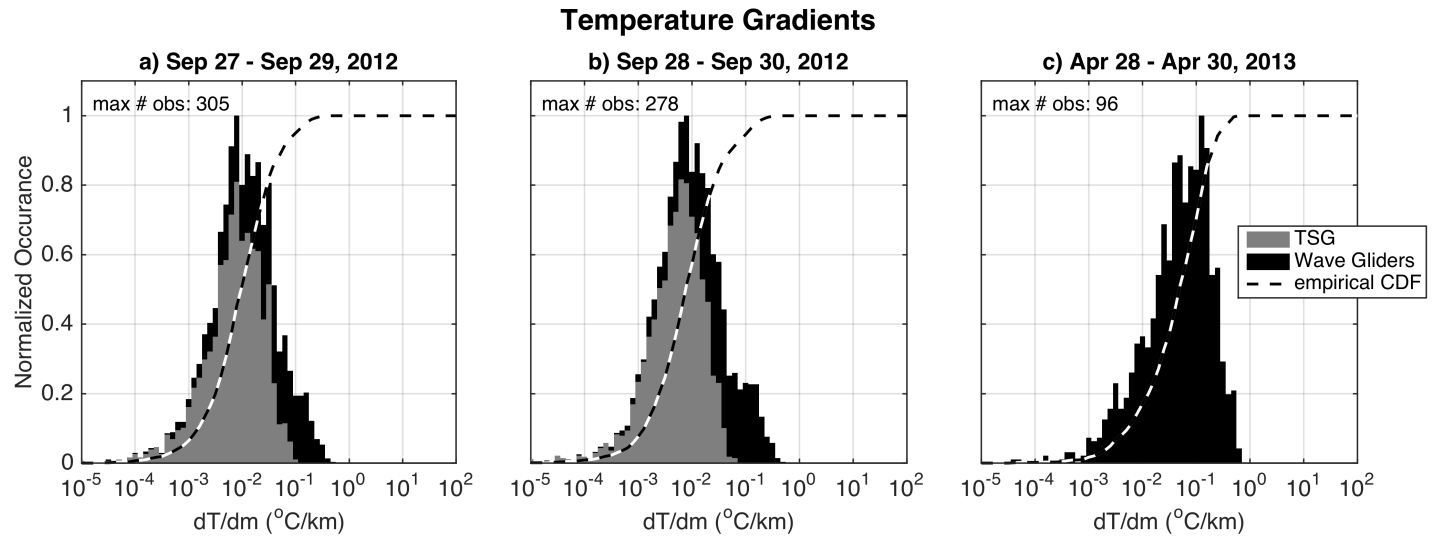

Figure 4-13: Normalized stacked histogram of observed temperature gradients over \pm 1 day for a) September 28, 2012, b) September 29, 2012, and c) April 29, 2013 from the shipboard thermosalinograph (when available, black) and Wave Gliders (grey). The black dotted line is the empirical cumulative distribution function. The gradients are calculated in the direction of the instrument movement, $m$. The normalization factor is included as the maximum number of observations in a bin.

\subsubsection{Advective effect on diurnal warming and heat flux}

Advection will act on any tracer, including temperature. However, it is far more difficult to directly separate temperature changes due to advection from the dominant diurnal warming signal at the surface, especially on the three days in question. For each case study, the peak change in salinity over the observed time, $\Delta t_{\max }$, (Table 4.1) is used to estimate an "observed salinity gradient" (see previous section). Likewise, the observed temperature gradients in time and space (Fig. 4-13) can be used to estimate the possible change in temperature due to advection. This provides some measure of possible temperature change due to advection. The temperature gradients were rarely density compensated in the region and thus, a simple relationship between temperature and salinity gradients is not expected.

Using the mean temperature gradients for each day, the estimated change in the peak warming is $0.05^{\circ} \mathrm{C}$. The directions of the current and gradient would determine if the change in temperature would increase or decrease the warming amount. Although the change in temperature due to advection seems small, the horizontal heat flux in the mixed layer $(H)$ can be estimated as

$$
H=\rho c_{p} \kappa_{\mathrm{eff}}|\nabla T| \frac{\Delta t}{24 \mathrm{hrs}}
$$

where $\rho$ is the surface density, $c_{p}$ is the specific heat capacity of seawater, and $|\nabla T|$ is the 
magnitude of the temperature gradient. Using a 4 hour advective time-scale and $\kappa_{\text {eff }}=1$

$\mathrm{m}^{2} / \mathrm{s}$, for the range of observed temperature gradients, the heat flux is $1-100 \mathrm{~W} / \mathrm{m}^{2}$ across the mixed layer. The heat flux associated with effective diffusivity from shear dispersion can be the same order of magnitude as the net surface heat flux. Here, the diffusivity is used as a proxy for advection over a day. Although diurnal warming is generally treated as a one-dimensional problem (e.g. Price et al., 1986; Kantha and Clayson, 1994; Gentemann et al., 2009; Bogdanoff et al., In Preparation), and in many cases that may be a correct assumption, in regions with relatively strong temperature gradients, near-surface advection may enhance or suppress diurnal warming and be an important source mechanism for lateral heat transport in the mixed layer.

\subsection{Summary \& Discussion}

The main points of this study can be summarized as follows:

1. DWL shear associated with momentum trapped near the ocean surface by the DWL creates vertical shear across the mixed layer and is hypothesized to be responsible for the observed near-surface freshening not associated with local precipitation.

2. The cycle of advection due to DWL shear combined with nocturnal convection creates an effective diffusion on the scale of 1 to $10 \mathrm{~km}$. Effective diffusion coefficients range from 1 to $250 \mathrm{~m}^{2} / \mathrm{s}$ and are a function of the time-scale of advection, the speed of the DWL current, and depth of the DWL compared to the mixed layer depth. The diffusivities associated with this mechanism are on the order of published estimated submesoscale diffusivities.

3. The near-surface advection associated with DWL shear may be a mechanism to suppress or enhance the diurnal change in temperature observed at a particular location, and a mechanism for lateral heat transport in the mixed layer.

The cycle can be summarized as follows. A precipitation event occurs creating an upper ocean horizontal gradient of salinity. Advection due to the vertical shear associated with the DWL current laterally transports water with a different salinity atop the rest of the mixed layer. Nocturnal convection mixes the advected water into the mixed layer. The advection and mixing mechanism can repeat until the gradient is no longer present. 


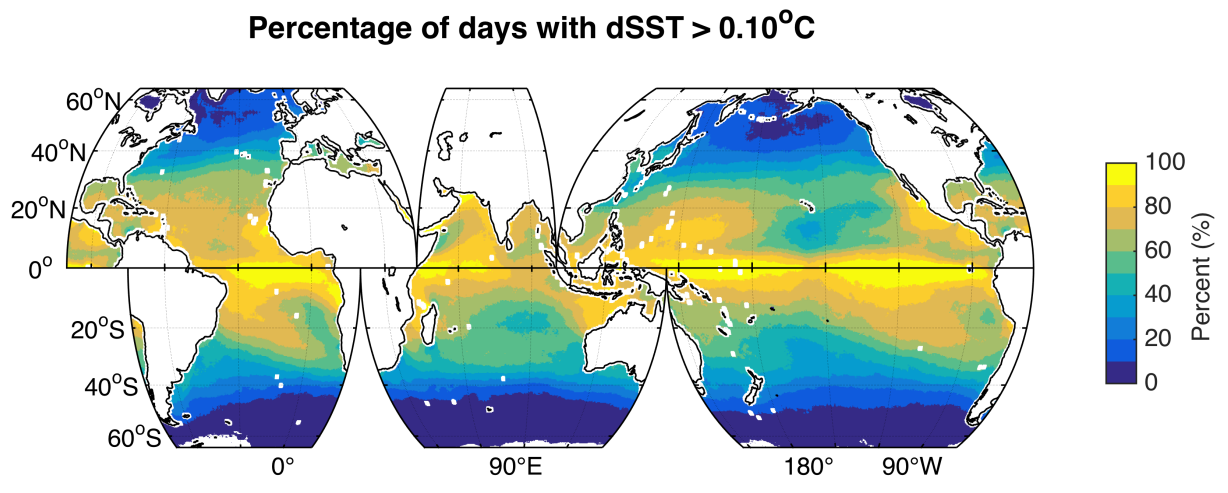

Figure 4-14: Global percentage of days with diurnal warming greater than $0.1^{\circ} \mathrm{C}$ from the Clayson diurnal warming parameterization of Bogdanoff et al. (In Preparation) over the 10 year periods from January 1, 1998 - December 31, 2007.

While near-surface freshening is only observed on a few days (out of a yearlong record) with strong diurnal warming and strong salinity gradients near the SPURS-I central mooring, a DWL current should be expected to occur on any day with diurnal warming. This study examines three particular days at a particular location, but there are global implications for mixing. Figure 4-14 shows a global picture of the estimated percentage of days with parameterized diurnal warming greater than $0.1^{\circ} \mathrm{C}$ (from Bogdanoff et al., In Preparation). Over a large portion of the globe some diurnal warming is expected on most days.

The global map of percentage of days with diurnal warming greater than $0.1^{\circ} \mathrm{C}$ is on an annual basis, but there is a seasonality to the frequency and magnitude of diurnal warming (Stuart-Menteth et al., 2003). Specifically, in winter time the diurnal cycle is suppressed as incoming solar radiation is at a minimum. There is also seasonality to submesoscale processes with reduced activity in the summertime (Callies et al., 2015). Interestingly, the summertime period of reduced submesoscale activity coincides with a time period of higher likelihood of diurnal warming occurrence. The effective mixing by DWL shear is a submesoscale process (order 1-10 km). However, even current high-resolution models runs will not likely properly resolve the near-surface stratification associated with diurnal warming. As global model resolution improves, submesoscale processes cannot be properly represented without resolving real processes such as the near-surface advection of DWL shear.

The observed and modeled cycle can be a mechanism of effective lateral mixing of any 
mixed layer tracer including those relevant to the biology and chemistry of the ocean. While here we focus on temperature and salinity, any passive tracer will be advected by the DWL current. Additionally, as horizontal model resolution is improved, it is important to consider necessary vertical resolution as well. Vertical resolution of less than $1 \mathrm{~m}$ is likely important to resolving strong diurnal warming events and DWL shear, although attempts are being made to parameterize warming in global climate models (Large and Caron, 2015).

The importance of resolving the diurnal cycle of the near-surface ocean and the subsequent phenomena associated with the warming is becoming increasingly clear with studies such as this one, Clayson and Bogdanoff (2013), and Seo et al. (2014). While it is challenging to separate out potential effects of advection due to the dominance of the diurnal cycle of temperature in the upper ocean, DWL shear can enhance or suppress the signal of warming in the near-surface ocean, and may be an important source of lateral heat transport in the upper ocean. This possible enhancement may account for some of the large diurnal warming observed, such as Gentemann et al. (2008). 


\section{Chapter 5}

\section{Conclusion}

The Salinity Processes in the Upper ocean Regional Study (SPURS-I) field campaign in the salinity maximum in the North Atlantic Ocean collected a new observational dataset of the upper ocean. This dissertation examines the turbulence observations provided by the Slocum gliders, as well as the suite of physical variables from moving platform allowing for the examination of temporal and spatial variability around the central mooring. This dissertation provides a new viewpoint of the ocean surface boundary layer, specifically the Diurnal Warm Layer (DWL) as a dynamic and complex region to characterize.

\subsection{Contributions}

Diurnal variability is important to resolve as a fundamental forcing of the Earth's climate system. There are several salient contributions this dissertation makes to our understanding of the upper ocean and physics of DWLs. Chapter 2 considered the turbulence observation, modeling, and theory of the DWL. The important contributions of this chapter include:

1. the observed enhanced turbulent kinetic energy dissipation during the daytime is associated with DWLs. The daily cycle of dissipation is oppositely phased from previous studies (e.g. Soloviev and Vershinsky, 1982; Soloviev et al., 1988; Moum et al., 1989; Brainerd and Gregg, 1993a,b), with the largest values coincident with the peak warming.

2. current one-dimensional ocean models do not resolve the enhanced turbulent kinetic energy dissipation. Neither the Kantha-Clayson model (a 2nd-momentum turbulence 
closure model) nor the Price-Weller-Pinkel (a dynamical instability model) reproduce the signal.

3. similarity theory, such as the Monin-Obukhov Similarity Theory, does not properly describe the enhanced daytime dissipation. It does however, properly describe the turbulence of the near-surface ocean under unstable forcing conditions.

4. DWLs are associated with shear across the layer consistent with previous observations (e.g. Price et al., 1986; Kudryavtsev and Soloviev, 1990), but there is not enough energy in the mean shear to explain the observed dissipation.

5. surface forcing alone cannot explain the observed enhanced turbulent kinetic energy dissipation.

6. the highest values of dissipation are coincident with the most stable conditions.

Overall, the observations reveal a pattern that is different than previously observed, and surface forcing is not the cause alone. Since surface forcing alone cannot describe the turbulent kinetic energy dissipation, it is important to consider alternative sources. Coincident variability in temperature and salinity is consistent with the existence of internal waves. Chapter 3 quantitatively demonstrates that internal waves exist within the DWL, and shows that internal waves are important to the structure and variability of the nearsurface ocean. In summary, the major conclusions are:

1. the conditions of the upper ocean during the daytime are favorable for internal waves, and the observed higher-than-expected TKE dissipation is associated with the strongest near-surface stable layers.

2. that internal waves are an important characteristic of DWLs (stable layers within the ocean boundary layer), much like in the atmospheric stable boundary layer.

3. deep ocean internal waves propagating up into the mixed layer can cause large horizontal velocities in the mixed layer.

4. that the inclusion of a DWL substantially changes propagation characteristics of internal waves from the deep ocean, and can cause shear across the DWL capable of generating a shear instability. 
5. increasing near-surface stratification can cause larger shear across the DWL due to internal waves, which is consistent with larger turbulent kinetic energy dissipation associated with larger stratification.

The concept of internal waves propagating from the deep ocean into the surface ocean, and then breaking and causing turbulence, is novel but consistent with the observations. It is further evidence that this is a dynamically and physically important region of the ocean to characterize. In addition, DWLs can rectify into longer time-scales and spatial-scales through the submesoscale mixing mechanism considered in Chapter 4. Advection in the near-surface during the daytime converts horizontal gradients into vertical ones. Coupled with vertical mixing at night within the mixed layer, it is a mechanism for effective lateral mixing. The salient conclusions of that chapter are:

1. the near-surface freshening that was observed on 3 different days during the SPURSI field campaign is associated with relatively strong diurnal warming. The cause is likely advection due to DWL shear associated with momentum trapped near the ocean surface by the DWL.

2. the cycle of advection due to DWL shear combined with nocturnal convection creates an effective diffusion on the scale of 1 to $10 \mathrm{~km}$. Effective diffusion coefficients range from 1 to $250 \mathrm{~m}^{2} / \mathrm{s}$ and are a function of the time-scale of advection, the speed of the DWL current, and depth of the DWL compared to the mixed layer depth. The diffusivities associated with this mechanism are on the order of published estimated submesoscale diffusivities.

3. the near-surface advection associated with DWL shear may be a mechanism to suppress or enhance the diurnal change in temperature observed at a particular location, and may also be a mechanism for lateral heat transport in the mixed layer.

The concept of a relatively calm stable layer due to diurnal warming is abrogated by the observations, modeling, and theory of this dissertation. A DWL is caused by momentum from the wind trapped in the very near the surface, creating a sheared layer. Internal waves propagating along the DWL can become unstable under certain conditions and generate small-scale turbulence. The layer can also rectify into the longer time-scales laterally smoothing ocean tracer gradients on the scale of 1-10 kilometers. 


\subsection{Implications}

The observations and results of this work open many questions about our understanding of the ocean boundary layer on a variety of time and spatial scales. Under conditions of relatively low wind speed and high solar insolation DWLs can exist from the surface of the ocean and extend down tens of centimeters to meters. Since the diurnal cycle of TKE dissipation observed during SPURS-I is opposite of the cycle observed by previous studies, there are broader implications for mixing within the boundary layer. Questions remain about the ubiquity of the enhanced TKE dissipation and dissipation of thermal variance in the daytime upper ocean. More observations are needed under a variety of geographic regions, seasonal time periods, and meteorological conditions.

While internal waves are likely responsible for the enhanced TKE dissipation observed in the DWL, questions remain about how this might translate into mixing (a diffusivity, for example). Although the TKE dissipation values are large, the implied mixing is not necessarily. Large mixing would wipe out the strong near-surface temperature gradients associated with DWLs. Since strong gradients persist throughout the day, mixing is likely suppressed under low wind speed conditions. In addition, a turbulent diffusivity calculated using the observed TKE dissipation and estimate of the buoyancy frequency (Osborn, 1980), would be near molecular levels under the observed enhanced TKE dissipation conditions. This is consistent with necessary levels to maintain a strong temperature gradient near the surface. Overall, there is likely weak mixing associated with the strong TKE dissipation.

The geographic pattern of the enhanced TKE dissipation due to deep internal waves propagating into the DWL has two primary factors: the depth of the seasonal thermocline and the likelihood of diurnal warming. Both are shown in Fig. 5.1. In general, this mechanism is likely important in regions with shallow mixed layer depths, which is a good proxy for the depth of the seasonal thermocline, and a strong likelihood of diurnal warming, such as the tropics and subtropics (including the region where the SPURS-I field campaign was located). The ubiquity of the mechanism must be tested across differing seasonal thermoclines depths.

Current one-dimensional mixed layer models do not reproduce the TKE dissipation, but do reproduce temperature and salinity signals fairly well. This implies that while the models do well with the physical tracers of the ocean, they do not contain the physics of the process 
a) Percentage of days with dSST $>0.10^{\circ} \mathrm{C}$

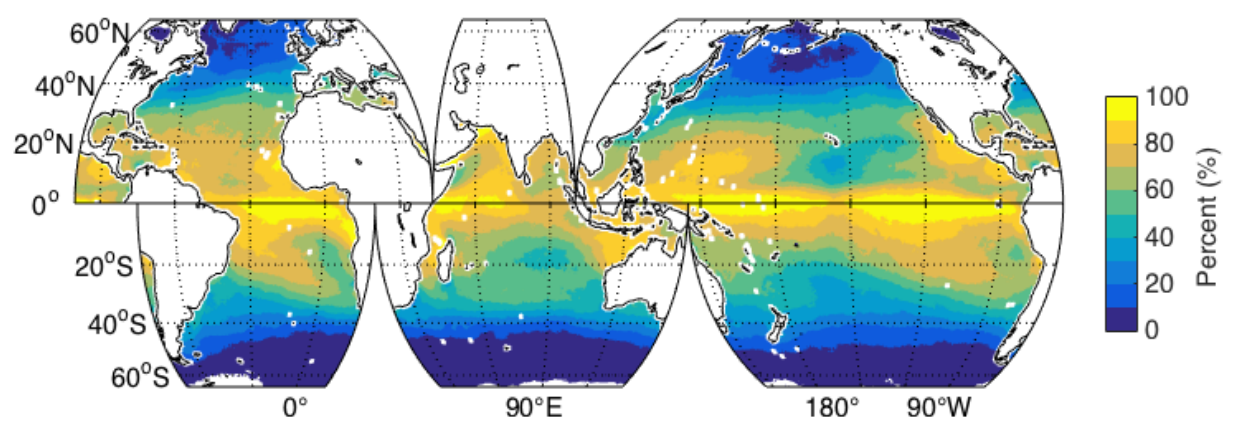

b) Annual-Averaged Mixed Layer Depth

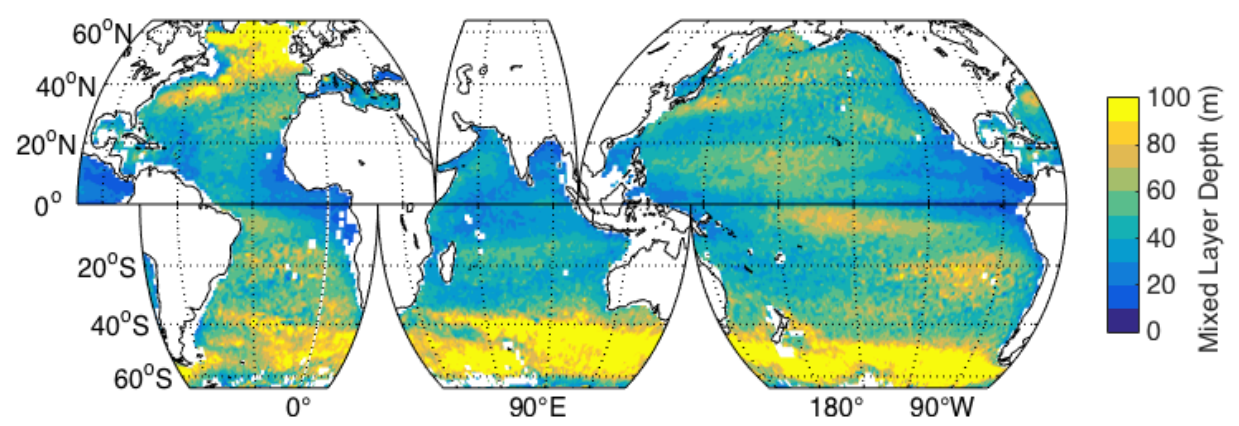

Figure 5-1: Global a) percentage of days with diurnal warming greater than $0.1^{\circ} \mathrm{C}$ from the Clayson diurnal warming parameterization of Bogdanoff et al. (In Preparation) over the 10 year periods from January 1, 1998 - December 31, 2007; b) annual averaged mixed layer depth based on the Holte and Talley (2009) Argo float climatology. 
responsible for the turbulence and TKE dissipation, such as internal waves. Additionally, it implies that mixing levels are likely fairly well represented in the models, even if they do not reproduce the enhanced TKE dissipation. While resolving those processes may not be important to properly modeling the mean temperature and salinity values, there will be broader implications for the energy balance in the ocean. When considering threedimensional models, the vertical and horizontal grid size choice will affect the magnitude of the submesoscale mixing associated with DWLs. It will be necessary to do studies to understand the necessary vertical and horizontal spacing to properly resolve these processes, as discussed in Chapter 4 .

Lastly, the concept of internal waves propagating from the deep ocean and generating turbulence near the surface has significant implications for ocean energy balance. DWLs coupling with internal waves is a mechanism to move deep ocean energy into the ocean surface and mixed layer. Generally, the boundary layer has been thought of as a source region for internal waves rather than a sink. Far more observations and modeling are necessary to explore this idea.

Overall, DWLs are active regions that are important to consider with implications for air-sea interaction estimates, oceanic turbulent energy balance, internal wave propagation and mixing, and submesoscale mixing.

\subsection{Future Work}

There are still significant questions that remain about the near-surface ocean under a variety of conditions. The importance of the DWL is clear, but there are several other factors not discussed in this dissertation that are important to consider, such as importance of surface gravity waves and so-called Langmuir turbulence, the variability of penetrative solar radiation, and the next steps to properly model the oceanic boundary layer.

The results of this dissertation demonstrate the need for higher resolution observations of temperature, salinity, and currents in the near-surface ocean across the range of possible atmospheric conditions. The time period of the SPURS-I field campaign provided limited observations of windy conditions. While the observations from the SPURS-I field campaign (Farrar et al., 2015) are arguably among the highest temporal- and vertical-resolution from a field campaign, many of the conclusions were observation-limited. Specifically, measure- 
ments of the near-surface currents are needed, but are also hampered by motion associated with surface gravity waves. The SPURS-II follow-on campaign will provide additional observations of the near-surface ocean under a variety of conditions (including geographic regions with different mixed layer depths and likelihood of diurnal warming, see Fig. 5.1), including attempts to obtain higher-resolution observations of upper ocean currents. Additionally, higher resolution observations of currents, temperature, and salinity in vertical and horizontal space and time will provide greater insight into the importance of advection in the near-surface ocean, and allow for better estimates of the effect on diurnal warming and horizontal mixing in the upper ocean.

\subsubsection{Langmuir turbulence and surface gravity waves}

Surface gravity waves, which nearly constantly exist in the ocean, can generate turbulence through Stokes drift, energizing turbulence in the upper ocean (D'Asaro et al., 2014). The importance of non-breaking waves on the enhancement of turbulence in the upper ocean is still unknown under many conditions due to lack of observations. Here, we only cursorily consider surface gravity waves and Langmuir cells. A shear production term of TKE can be considered for Langmuir turbulence (Belcher et al., 2012).

Several Langmuir turbulence studies explored cases in which the surface layer is weakly stratified (e.g. McWilliams et al., 1997). However, under conditions of very stable stratification, such as DWLs, the effect is still unknown. Continued improvement of parameterizations of wave effects are being considered (Kantha and Clayson, 2004; Harcourt, 2015). For example, the Langmuir number under the low wind conditions is $\sim 0.3$, which is consistent with estimates from Belcher et al. (2012). However, a negative Langmuir Stability Length is calculated under conditions of stable surface forcing, which has an unclear meaning.

Additionally, one must be careful in how wave-turbulence interaction is considered (Kantha et al., 2014). There is enough energy in surface gravity waves to explain the enhanced TKE dissipation, so it is important to consider as a source of energy. However, the possible mechanism for turbulent transfer remains unclear for non-breaking waves. For example, Babanin (2006) considers a wave-amplitude-based Reynolds number, which may allow orbital motion to generate turbulence however, further verification is needed. The possible mechanism remains unclear. There are studies beginning to explore Langmuir cells under stabilizing surface forcing Pearson et al. (2015), but even these studies still require relatively 
strong wind speed for Langmuir cell development.

\subsubsection{Penetrative solar radiation, and biophysical-chemical interaction}

Oceanographers have long desired to measure solar radiation within the ocean and parameterize the attenuation (e.g., Jerlov, 1951). One of the first standardized ways of measuring the optical properties of the ocean was the Secchi disk (Wernand, 2010). Since visible radiation can be estimated with traditional Secchi disks and is the portion of the spectrum most important to life within the ocean, it is generally the most studied. While infrared (IR) experiments exist, traditional experiments and parameterizations (Jerlov, 1976) focus on the visible region of the spectrum since it penetrates much deeper in the ocean than IR, and is easier to measure.

While our instruments have become more complex and accurate, parameterizations are still used to obtain a best guess of solar extinction where direct observations are not available. Integrated photosythetically-active radiation (PAR or visible radiation, integrated across $400-700 \mathrm{~nm}$ ) is a commonly measured quantity in biological and chemical studies (in addition to many physical studies), and thus many parameterizations focus on the attenuation of PAR (Wei and Lee, 2013). However, especially for physical process studies, it is important to accurately measure the entire spectrum of solar radiation (Simpson and Dickey, 1981). Processes sensitive to ultraviolet (UV) radiation are also limited by general observations of PAR, with attenuation depths ranging from centimeters to 10 meters (Morris et al., 1995). Attenuation across the entire solar spectrum is not constant, nor is attenuation constant within the bands of the solar spectrum (UV, PAR/visible, and IR).

Attenuation of solar radiation within an ocean column will change based on several factors including wavelength and the amount of dissolved organic carbon, phytoplankton chlorophyll, total suspended solids, and turbulence in the water column. In general, the Inherent Optical Properties (IOPs), such as chlorophyll amount, set the Apparent Optical Properties (AOPs) for the water column, such as the measured solar attenuation. While the distinguishing factors may be subtle, converting from AOPs to IOPs and vice versa can be difficult (Bracchini et al., 2009). It is simplest to measure downwelling diffuse irradiance, as is done through integrated PAR sensors, which can be used to retrieve an AOP of the water column, such as an observed diffuse attenuation coefficient.

Measuring the IR component of solar radiation is important to heating in the upper 
ocean. The diurnal cycle of sea surface temperature, diurnal near-surface stable boundary layers, and upper ocean dynamics are sensitive to the extinction of solar radiation. No current observational platform or instrumentation can provide all of the necessary information for accurate modeling and characterization of the near-surface layer of the ocean. Differences in current parameterization have implications for all processes that require an accurate estimates of available solar radiation within the water column. There is also a great opportunity for the development of better observational tools to understand the variability of solar extinction.

\subsubsection{Modeling the ocean boundary layer}

As the resolution of ocean models becomes greater in horizontal and vertical space, and time, the importance of properly resolving and understanding processes associated with stable boundary layer and diurnal warming will be even more important. As recent studies have shown, including a diurnal cycle is important to lower frequency physical processes (Clayson and Weitlich, 2007; Woolnough et al., 2007; Seo et al., 2014; Tseng et al., 2014).

There are two clear next steps in modeling. The first step is to run a three-dimensional non-hydrostatic model with high enough resolution in space and time to resolve DWLs. Considering cases with and without a diurnal cycle, such as was done by Clayson and Bogdanoff (2013) for air-sea fluxes, will allow for the exploration of the importance of DWLs in submesoscale lateral mixing. This model will have to be run over tens of kilometers in space, but only slightly deeper than the mixed layer. The second would be to run a fully non-hydrostatic model over a large enough space to consider deep internal waves propagating from the stratified interior into the DWL. Modeling internal waves in regions of complex stratification is still an area of current research (Mathur and Peacock, 2009). This poses significant problems, as the model will have to be run at high-enough resolution to resolve turbulence, but also have a large enough domain to capture long internal waves. A first attempt could be to consider internal waves with relatively small wavelengths, but those are not necessarily the waves that will tunnel through the remnant mixed layer. The model problem associated with the turbulence of breaking internal waves is likely far more challenging than exploring the submesoscale mixing mechanism. However, modeling is a logical next step. It is vital to consider diurnal variability and the implication of not properly resolving the diurnal cycle. There are plenty of open questions to answer, and many that 
remain to be discovered about the physics and dynamics of DWLs. 


\section{Appendix A}

\section{Derivation of turbulent kinetic energy dissipation in Price-Weller-Pinkel model}

Price-Weller-Pinkel (PWP) is a slab ocean model outlined in Price et al. (1986). Schudlich and Price (1992) diagnose the turbulent kinetic energy (TKE) dissipation $(\epsilon)$ in the PWP model however, the version of the model is designed for exploration of equatorial phenomena and is slightly different than the original version. What follows is the derivation to estimate TKE dissipation in the original model.

The equations of temperature, salinity, and momentum can be written in tensor notation with the Boussinesq approximation, noting that $\frac{\partial}{\partial x_{1}}=0$ and $\frac{\partial}{\partial x_{2}}=0$ :

$$
\begin{gathered}
\frac{\partial T}{\partial t}+u_{3} \frac{\partial T}{\partial x_{3}}=\kappa_{T} \frac{\partial^{2} T}{\partial x_{3}^{2}} \\
\frac{\partial S}{\partial t}+u_{3} \frac{\partial S}{\partial x_{3}}=\kappa_{S} \frac{\partial^{2} S}{\partial x_{3}^{2}} \\
\frac{\partial u_{i}}{\partial t}+u_{3} \frac{\partial u_{i}}{\partial x_{3}}=-\frac{1}{\bar{\rho}} \frac{\partial p}{\partial x_{3}}-\delta_{i 3} \frac{\rho}{\bar{\rho}} g+f \epsilon_{i j 3} u_{j}+\nu \frac{\partial^{2} u_{i}}{\partial x_{3}^{2}}
\end{gathered}
$$

where $T, S, u_{i}$ are the temperature, salinity, and velocity, respectively. The fluxes are considered as boundary conditions to the model and taken into account at the end of the 
derivation.

\section{A.1 Derivation of implied MKE \& TKE in PWP}

Utilizing continuity and the assumption of incompressibility, Eq. A.3 can be written as:

$$
\frac{\partial u_{i}}{\partial t}+\frac{\partial u_{i} u_{3}}{\partial x_{3}}-u_{i} \frac{\partial y_{3}}{\partial x_{3}}=-\frac{1}{\bar{\rho}} \frac{\partial p}{\partial x_{3}}-\delta_{i 3} \frac{\rho}{\bar{\rho}} g+f \epsilon_{i j 3} u_{j}+\nu \frac{\partial^{2} u_{i}}{\partial x_{3}^{2}}
$$

The initial step will be expanding the momentum equation into mean and turbulent parts. First, though, the shear terms will be defined as:

$$
\nu \frac{\partial^{2} u_{i}}{\partial x_{3}^{2}}=\frac{\partial \sigma_{i 3}}{\partial x_{3}}, \text { where } \sigma_{i 3}=2 \nu s_{i 3}=\nu\left(\frac{\partial u_{3}}{\partial x_{i}}+\frac{\partial u_{i}}{\partial x_{3}}\right)
$$

PWP does not explicitly have turbulent components. The assumption is that the values at the start or end of each time step are the mean values, and the unresolved fluctuations inside the time step are turbulent. The variables of the model can be broken into mean and turbulent parts:

$$
\begin{gathered}
T=\bar{T}+T^{\prime} \\
S=\bar{S}+T^{\prime} \\
\rho=\bar{\rho}+\rho^{\prime} \\
p=\bar{p}+p^{\prime} \\
u_{1}=\bar{u}_{1}+u_{1}^{\prime} \\
u_{2}=\bar{u}_{2}+u_{2}^{\prime} \\
u_{3}=u_{3}^{\prime}
\end{gathered}
$$


The model does not have a mean vertical velocity.

$$
\begin{aligned}
\frac{\partial}{\partial t}\left(\bar{u}_{i}+u_{i}^{\prime}\right)+ & \frac{\partial}{\partial x_{3}}\left(\left(\bar{u}_{i}+u_{i}^{\prime}\right)\left(\bar{u}_{3}+u_{3}^{\prime}\right)\right)= \\
& -\frac{1}{\bar{\rho}} \frac{\partial}{\partial x_{3}}\left(\bar{p}+p^{\prime}\right)-\delta_{i 3} \frac{g}{\bar{\rho}}\left(\bar{\rho}+\rho^{\prime}\right)+f \epsilon_{i j 3}\left(\bar{u}_{j}+u_{j}^{\prime}\right)+\frac{\partial}{\partial x_{3}}\left(\bar{\sigma}_{i 3}+\sigma_{i 3}^{\prime}\right)
\end{aligned}
$$

Expanding out further and noting that $\bar{u}_{3}=0$ :

$$
\begin{aligned}
\frac{\partial \bar{u}_{i}}{\partial t}+\frac{\partial u_{i}^{\prime}}{\partial t}+ & \frac{\partial \bar{u}_{i} u_{3}^{\prime}}{\partial x_{3}}+\frac{\partial u_{i}^{\prime} u_{3}^{\prime}}{\partial x_{3}}= \\
& -\frac{1}{\bar{\rho}} \frac{\partial \bar{p}}{\partial x_{3}}-\frac{1}{\bar{\rho}} \frac{\partial p^{\prime}}{\partial x_{3}}-\delta_{i 3} g-\delta_{i 3} \frac{\rho^{\prime}}{\bar{\rho}} g+f \epsilon_{i j 3} \bar{u}_{j}+f \epsilon_{i j 3} u_{j}^{\prime}+\frac{\partial \bar{\sigma}_{i 3}}{\partial x_{3}}+\frac{\partial \sigma_{i 3}^{\prime}}{\partial x_{3}}
\end{aligned}
$$

Reynolds averaging provides the Mean Momentum Equation:

$$
\frac{\partial \bar{u}_{i}}{\partial t}+\frac{\partial \overline{u_{i}^{\prime} u_{3}^{\prime}}}{\partial x_{3}}=-\frac{1}{\bar{\rho}} \frac{\partial \bar{p}}{\partial x_{3}}-\delta_{i 3} g+f \epsilon_{i j 3} \bar{u}_{j}+\frac{\partial \bar{\sigma}_{i 3}}{\partial x_{3}}
$$

Subtracting the Mean Momentum Equation (A.9) from the total (A.8) yields the Turbulent Momentum Equation:

$$
\frac{\partial u_{i}^{\prime}}{\partial t}+\frac{\partial \bar{u}_{i} u_{3}^{\prime}}{\partial x_{3}}+\frac{\partial u_{i}^{\prime} u_{3}^{\prime}}{\partial x_{3}}-\frac{\partial \overline{u_{i}^{\prime} u_{3}^{\prime}}}{\partial x_{3}}=-\frac{1}{\bar{\rho}} \frac{\partial p^{\prime}}{\partial x_{3}}-\delta_{i 3} \frac{\rho^{\prime}}{\bar{\rho}} g+f \epsilon_{i j 3} u_{j}^{\prime}+\frac{\partial \sigma_{i 3}^{\prime}}{\partial x_{3}}
$$

A Mean Kinetic Energy (MKE, denoted as E) equation (or mean Kinetic Energy of the Mean Flow) is derived by multiplying the Mean Momentum Equation by the mean velocity:

$$
\begin{aligned}
& \bar{u}_{i} \frac{\partial \bar{u}_{i}}{\partial t}+\bar{u}_{i} \frac{\partial \overline{u_{i}^{\prime} u_{3}^{\prime}}}{\partial x_{3}}=-\bar{u}_{i} \frac{1}{\bar{\rho}} \frac{\partial \bar{p}}{\partial x_{3}}-\bar{u}_{i} \delta_{i 3} g+f \epsilon_{i j 3} \bar{u}_{j}+\bar{u}_{i} \frac{\partial \bar{\sigma}_{i 3}}{\partial x_{3}} \\
& \frac{\partial}{\partial t}\left(\frac{1}{2} \bar{u}_{i}^{2}\right)+\bar{u}_{i} \frac{\partial \overline{u_{i}^{\prime} u_{3}^{\prime}}}{\partial x_{3}}=-\bar{u}_{3} \frac{1 \partial \bar{p}^{0}}{\rho}-\bar{u}_{i \delta_{i 3}}+\frac{0}{\partial x_{3}}+\underline{\epsilon \epsilon_{i j 3} \bar{u}_{i} \vec{u}_{j}}+\frac{\partial \bar{u}_{i} \bar{\sigma}_{i 3}}{\partial x_{3}}-\bar{\sigma}_{i 3} \frac{\partial \bar{u}_{i}}{\partial x_{3}} \\
& \frac{\partial}{\partial t}\left(\frac{1}{2} \bar{u}_{i}^{2}\right)+\frac{\partial \bar{u}_{i} \overline{u_{i}^{\prime} u_{3}^{\prime}}}{\partial x_{3}}-\overline{u_{i}^{\prime} u_{3}^{\prime}} \frac{\partial \bar{u}_{i}}{\partial x_{3}}=2 \nu \frac{\partial \bar{u}_{i} \bar{s}_{i 3}}{\partial x_{3}}-2 \nu \bar{s}_{i 3} \frac{\partial \bar{u}_{i}}{\partial x_{3}} \\
& \frac{\partial E}{\partial t}=\underbrace{\frac{\partial}{\partial x_{3}}\left(2 \nu \bar{u}_{i} \bar{s}_{i 3}-\bar{u}_{i} \overline{u_{i}^{\prime} u_{3}^{\prime}}\right)}_{\text {transport }}-\underbrace{2 \nu \bar{s}_{i 3} \bar{s}_{i 3}}_{\text {diss, }(\bar{\epsilon})}+\underbrace{\overline{u_{i}^{\prime} u_{3}^{\prime}} \frac{\partial \bar{u}_{i}}{\partial x_{3}}}_{\text {loss to turb }}
\end{aligned}
$$

Each term is label above. This is the MKE equation for the PWP model.

To get the Turbulent Kinetic Energy (TKE) Equation (which is the mean Kinetic Energy 
of the Turbulent Flow), the turbulent momentum equation is multiplied by $u_{i}^{\prime}$ :

$$
u_{i}^{\prime} \frac{\partial u_{i}^{\prime}}{\partial t}+u_{i}^{\prime} \frac{\partial \bar{u}_{i} u_{3}^{\prime}}{\partial x_{3}}+u_{i}^{\prime} \frac{\partial u_{i}^{\prime} u_{3}^{\prime}}{\partial x_{3}}-u_{i}^{\prime} \frac{\partial \overline{u_{i}^{\prime} u_{3}^{\prime}}}{\partial x_{3}}=-\frac{u_{i}^{\prime}}{\bar{\rho}} \frac{\partial p^{\prime}}{\partial x_{3}}-\delta_{i 3} u_{i}^{\prime} \frac{\rho^{\prime}}{\bar{\rho}} g+. f \epsilon_{i j 3} u_{i}^{\prime} u_{j}^{\prime}+u_{i}^{\prime} \frac{\partial \sigma_{i 3}^{\prime}}{\partial x_{3}}
$$

Using the chain rule, TKE equation can be written as:

$$
\begin{aligned}
\frac{\partial}{\partial t}\left(\frac{1}{2} u_{i}^{\prime 2}\right)+u_{i}^{\prime} \frac{\partial \bar{u}_{i} u_{3}^{\prime}}{\partial x_{3}}+\frac{\partial\left(\frac{1}{2} u_{i}^{\prime} u_{i}^{\prime} u_{3}^{\prime}\right)}{\partial x_{3}}- & \frac{\partial u_{i}^{\prime} \overline{u_{i}^{\prime} u_{3}^{\prime}}}{\partial x_{3}}+\overline{u_{i}^{\prime} u_{3}^{\prime}} \frac{\partial u_{i}^{\prime}}{\partial x_{3}}= \\
& -\frac{1}{\bar{\rho}} \frac{\partial u_{i}^{\prime} p^{\prime}}{\partial x_{3}}-\delta_{i 3} u_{i}^{\prime} \frac{\rho^{\prime}}{\bar{\rho}} g+\frac{\partial \sigma_{i 3}^{\prime} u_{i}^{\prime}}{\partial x_{3}}-\sigma_{i 3}^{\prime} \frac{\partial u_{i}^{\prime}}{\partial x_{3}}
\end{aligned}
$$

Focusing on the second term on the left-hand side, using the fact that $\bar{s}_{i 3}=\frac{\partial \bar{u}_{i}}{\partial x_{3}}$, and rewriting the shear terms (A.5 and last two terms in the equation above) yields:

$$
u_{i}^{\prime} \frac{\partial \bar{u}_{i} u_{3}^{\prime}}{\partial x_{3}}=u_{i}^{\prime} u_{3}^{\prime} \frac{\partial \bar{u}_{i}}{\partial x_{3}}+u_{i}^{\prime} \bar{u}_{i} \frac{\partial u^{\prime} / 3}{\partial x_{3}}=u_{i}^{\prime} u_{3}^{\prime} \bar{s}_{i 3}
$$

and

$$
\begin{aligned}
\frac{\partial}{\partial t}\left(\frac{1}{2} u_{i}^{\prime 2}\right)+u_{i}^{\prime} u_{3}^{\prime} \bar{s}_{i 3}+\frac{\partial\left(\frac{1}{2} u_{i}^{\prime} u_{i}^{\prime} u_{3}^{\prime}\right)}{\partial x_{3}}- & \frac{\partial u_{i}^{\prime} \overline{u_{i}^{\prime} u_{3}^{\prime}}}{\partial x_{3}}+\overline{u_{i}^{\prime} u_{3}^{\prime}} \frac{\partial u_{i}^{\prime}}{\partial x_{3}}= \\
& -\frac{1}{\bar{\rho}} \frac{\partial u_{i}^{\prime} p^{\prime}}{\partial x_{3}}-\delta_{i 3} u_{i}^{\prime} \frac{\rho^{\prime}}{\bar{\rho}} g+\frac{\partial 2 \nu s_{i 3}^{\prime} u_{i}^{\prime}}{\partial x_{3}}-2 \nu s_{i 3}^{\prime} s_{i 3}^{\prime}
\end{aligned}
$$

Reynolds averaging the above equation yields:

$$
\begin{aligned}
\frac{\partial}{\partial t}\left(\overline{\frac{1}{2} u_{i}^{\prime 2}}\right)+\overline{u_{i}^{\prime} u_{3}^{\prime}} \bar{s}_{i 3}+\frac{\partial\left(\frac{1}{2} \overline{u_{i}^{\prime} u_{i}^{\prime} u_{3}^{\prime}}\right)}{\partial x_{3}}-\frac{\partial u_{i}^{\prime} \overline{u_{i}^{\prime} u_{3}}}{\partial x_{3}}+\overline{u_{i}^{\prime} u_{3}^{\prime}} \frac{\partial \bar{u}_{i}^{\prime}}{\partial x_{3}}= \\
-\frac{1}{\bar{\rho}} \frac{\partial \overline{u_{i}^{\prime} p^{\prime}}}{\partial x_{3}}-\delta_{i 3} \frac{g}{\bar{\rho}} \overline{u_{i}^{\prime} \rho^{\prime}}+\frac{\partial}{\partial x_{3}}\left(2 \nu \overline{u_{i}^{\prime} s_{i 3}^{\prime}}\right)-2 \nu \overline{s_{i 3}^{\prime} s_{i 3}^{\prime}}
\end{aligned}
$$

Defining TKE as the variable e (which is mean TKE), and moving terms around yields:

$$
\frac{\partial e}{\partial t}+\frac{\partial\left(\frac{1}{2} \overline{u_{i}^{\prime} u_{i}^{\prime} u_{3}^{\prime}}\right)}{\partial x_{3}}=-\frac{1}{\bar{\rho}} \frac{\partial \overline{u_{i}^{\prime} p^{\prime}}}{\partial x_{3}}+\frac{\partial}{\partial x_{3}}\left(2 \nu \overline{u_{i}^{\prime} s_{i 3}^{\prime}}\right)-\delta_{i 3} \frac{g}{\bar{\rho}} \overline{u_{i}^{\prime} \rho^{\prime}}-\overline{u_{i}^{\prime} u_{3}^{\prime}} \bar{s}_{i 3}-\underbrace{2 \nu \overline{s_{i 3}^{\prime} s_{i 3}^{\prime}}}_{\epsilon}
$$


which can be written as:

$$
\frac{\partial e}{\partial t}=-\underbrace{\frac{\partial}{\partial x_{3}}\left(\frac{1}{2} \overline{u_{i}^{\prime} u_{i}^{\prime} u_{3}^{\prime}}+\frac{1}{\bar{\rho}} \overline{u_{i}^{\prime} p^{\prime}}-2 \nu \overline{u_{i}^{\prime} s_{i 3}^{\prime}}\right)}_{\text {transport }}-\underbrace{\delta_{i 3} \frac{g}{\bar{\rho}} \overline{u_{i}^{\prime} \rho^{\prime}}}_{\text {buoy prod }}-\underbrace{\overline{u_{i}^{\prime} u_{3}^{\prime}} \frac{\partial \bar{u}_{i}}{\partial x_{3}}}_{\text {shear prod }}-\underbrace{2 \nu \overline{s_{i 3}^{\prime} s_{i 3}^{\prime}}}_{\text {diss }(\epsilon)}
$$

Each term is defined above and the transport terms are in the flux divergence form. Note that the loss to turbulence term in Eq. A.14 is the shear production term in Eq. A.21.

\section{A.2 Estimation of TKE Dissipation}

Following Schudlich and Price (1992) during steady, homogenous, vertically sheared turbulence, the TKE dissipation rate can be determined through production dissipation balance (also assuming the transport due to turbulent fluctuations and transport due to the viscous stresses are small). Likewise, if the turbulent components only occur in between time steps, the change in TKE from time step to time step in zero. Therefore, if integration is done over the domain, transport terms should be zero as well.

$$
0=-\overline{u_{i}^{\prime} u_{3}^{\prime}} \bar{s}_{i 3}-\epsilon-\delta_{i 3} \frac{g}{\bar{\rho}} \overline{u_{i}^{\prime} \rho^{\prime}}
$$

Expanding the tensor notation across three dimensions yields:

$$
0=-\overline{u_{1}^{\prime} u_{3}^{\prime}} \bar{s}_{13}-\overline{u_{2}^{\prime} u_{3}^{\prime}} \bar{s}_{23}-\overline{u_{i}^{\prime} u_{3}^{\prime}} \bar{s}_{33}-\frac{0}{\bar{\rho}} \epsilon-\frac{g}{\overline{u_{3}^{\prime} \rho^{\prime}}}
$$

Rearranging leads to:

$$
\epsilon=-\overline{u_{1}^{\prime} u_{3}^{\prime}} \bar{s}_{13}-\overline{u_{2}^{\prime} u_{3}^{\prime}} \bar{s}_{23}-\frac{g}{\bar{\rho}} \overline{u_{3}^{\prime} \rho^{\prime}}
$$

Recall that $\bar{s}_{i 3}=\frac{\partial \bar{u}_{i}}{\partial x_{3}}$ :

$$
\epsilon=-\overline{u_{1}^{\prime} u_{3}^{\prime}} \frac{\partial \bar{u}_{1}}{\partial x_{3}}-\overline{u_{2}^{\prime} u_{3}^{\prime}} \frac{\partial \bar{u}_{2}}{\partial x_{3}}-\frac{g}{\bar{\rho}} \overline{u_{3}^{\prime} \rho^{\prime}}
$$

Utilizing the mean momentum equation for each dimension (eq. A.9), one can solve for $\overline{u_{1}^{\prime} u_{3}^{\prime}}$ and $\overline{u_{2}^{\prime} u_{3}^{\prime}}$ :

$$
\begin{aligned}
& \frac{\partial \overline{u_{1}^{\prime} u_{3}^{\prime}}}{\partial x_{3}}=-\frac{\partial \bar{u}_{1}}{\partial t}+f \bar{u}_{2}+\frac{\partial \bar{\sigma}_{13}}{\partial x_{3}}=-\frac{\partial \bar{u}_{1}}{\partial t}+f \bar{u}_{2}+\frac{1}{\bar{\rho}} \frac{\partial \bar{\tau}_{13}}{\partial x_{3}} \\
& \frac{\partial \overline{u_{2}^{\prime} u_{3}^{\prime}}}{\partial x_{3}}=-\frac{\partial \bar{u}_{2}}{\partial t}-f \bar{u}_{1}+\frac{\partial \bar{\sigma}_{23}}{\partial x_{3}}=-\frac{\partial \bar{u}_{2}}{\partial t}-f \bar{u}_{1}+\frac{1}{\bar{\rho}} \frac{\partial \bar{\tau}_{23}}{\partial x_{3}}
\end{aligned}
$$


Integrating the above equations will yield $\overline{u_{1}^{\prime} u_{3}^{\prime}}$ and $\overline{u_{2}^{\prime} u_{3}^{\prime}}$, where $z_{b}$ is the bottom of the model:

$$
\overline{u_{1}^{\prime} u_{3}^{\prime}}(z)-\overline{u_{1}^{\prime} u_{3}^{\prime}}\left(\overrightarrow{\left.z_{b}\right)}=\int_{z_{b}}^{z}-\frac{\partial \bar{u}_{1}}{\partial t}+f \bar{u}_{2}+\frac{1}{\bar{\rho}} \frac{\partial \bar{\tau}_{13}}{\partial x_{3}} d x_{3}\right.
$$

and likewise,

$$
\overline{u_{2}^{\prime} u_{3}^{\prime}}(z)=\int_{z_{b}}^{z}-\frac{\partial \bar{u}_{2}}{\partial t}-f \bar{u}_{1}+\frac{1}{\bar{\rho}} \frac{\partial \bar{\tau}_{23}}{\partial x_{3}} d x_{3}
$$

Now, using the linear equation of state $(\rho=\bar{\rho}(1+\alpha \Delta T+\beta \Delta S))$, the buoyancy flux equation is derived as:

$$
\frac{\partial \rho}{\partial t}+u_{3} \frac{\partial \rho}{\partial x_{3}}=\bar{\rho} \alpha\left(\frac{\partial T}{\partial t}+u_{3} \frac{\partial T}{\partial x_{3}}\right)+\bar{\rho} \beta\left(\frac{\partial S}{\partial t}+u_{3} \frac{\partial S}{\partial x_{3}}\right)=\frac{\alpha}{c_{p}} \frac{\partial I}{\partial x_{3}}+\bar{\rho} \beta \frac{\partial J}{\partial x_{3}}
$$

where $I$ is the surface heat flux (including penetrative solar radiation) and $J$ is the surface freshwater flux. Following the same steps as to get the mean momentum equation yields:

$$
\frac{\partial \bar{\rho}}{\partial t}+\frac{\partial \overline{u_{3}^{\prime} \rho}}{\partial x_{3}}=\frac{\alpha}{c_{p}} \frac{\partial \bar{I}}{\partial x_{3}}+\bar{\rho} \beta \frac{\partial \bar{J}}{\partial x_{3}}
$$

Therefore:

$$
\frac{\partial \overline{u_{3}^{\prime} \rho^{\prime}}}{\partial x_{3}}=-\frac{\partial \bar{\rho}}{\partial t}+\frac{\alpha}{c_{p}} \frac{\partial \bar{I}}{\partial x_{3}}+\beta \frac{\partial \bar{J}}{\partial x_{3}}=-\bar{\rho} \alpha \frac{\partial \bar{T}}{\partial t}-\bar{\rho} \beta \frac{\partial \bar{S}}{\partial t}+\frac{\alpha}{c_{p}} \frac{\partial \bar{I}}{\partial x_{3}}+\bar{\rho} \beta \frac{\partial \bar{J}}{\partial x_{3}}
$$

and,

$$
\frac{g}{\bar{\rho}} \overline{u_{3}^{\prime} \rho^{\prime}}(z)=\int_{z_{b}}^{z}\left[g \alpha\left(-\frac{\partial \bar{T}}{\partial t}+\frac{1}{\bar{\rho} c_{p}} \frac{\partial \bar{I}}{\partial x_{3}}\right)+g \beta\left(-\frac{\partial \bar{S}}{\partial t}+\frac{\partial \bar{J}}{\partial x_{3}}\right)\right] d x_{3}
$$

The integrals are computed numerically in the model and combined to estimate the TKE dissipation as in Eq. A.24. 


\section{Appendix B}

\section{Derivation of Internal Wave induced Horizontal Velocities}

Deep internal waves can induce horizontal velocities in the mixed layer as shown by D'Asaro (1978). We will include a diurnal warm layer to explore how a near-surface stratified layer can change the upward propagation characteristics of deep internal waves. We can re-derive the D'Asaro (1978) solution by taking the limit of the diurnal warm layer depth going to zero. The details of a linearized internal wave problem can be found in Gill (1982), Lighthill (1978), Kundu and Cohen (2008), Gerkema and Zimmerman (2008), or Sutherland (2010). The linearized vertical velocity equation for a three-dimensional system $(x, y, z)$ with no mean flow is

$$
\frac{\partial^{2}}{\partial t^{2}}\left(\nabla_{H}^{2} w^{\prime}+\frac{\partial^{2} w^{\prime}}{\partial z^{2}}\right)+f^{2} \frac{\partial^{2} w^{\prime}}{\partial z^{2}}+N^{2} \nabla_{H}^{2} w^{\prime}=0
$$

where $w^{\prime}$ is the perturbation vertical velocity, $f$ is the Coriolis parameter, $N$ is the BruntVäisälä or buoyancy frequency, and $\nabla_{H}^{2}$ is the horizontal Laplacian.

We will assume a wave form of $w^{\prime}(x, z, t)=W(z) e^{i(\alpha x-\omega t)}$. In this case, we are assuming the wave is propagating in the $x$-direction, which we can do this without any loss of generality. It will still induce a flow in $y$-direction. The $x$-direction can now be thought of as the direction of wave propagation. Thus, the linearized internal wave equation for a given density structure (here we will consider layers with a constant buoyancy frequency) is given by

$$
\frac{d^{2} W(z)}{d z^{2}}+\alpha^{2} \frac{N^{2}-\omega^{2}}{\omega^{2}-f^{2}} W(z)=0
$$


where $\alpha$ is the horizontal wavenumber in the direction of the wave propagation, $N$ is the buoyancy frequency of the layer, $f$ is the Coriolis parameter, and and $\omega$ is the frequency of the wave.

\section{B.1 Derivation with a diurnal warm layer}

We can consider a stratified near-surface layer atop a well-mixed remnant mixed layer. This is schematically shown in Fig. 3-8b. We consider the case without shear in the diurnal warm layer as that cannot be solved analytically given our setup. This provides three regions:

- Diurnal Warm Layer with $N=N_{d}$

- Remnant Mixed Layer with $N=0$

- Stratified Interior with $N=N_{0}$

Each region has a solution that will be matched using boundary conditions at the surface with a rigid lid, $W(0)=0$, and at the two interfaces (vertical motion is continuous across the interfaces, but horizontal motion is not). In the diurnal warm layer (DWL):

$$
\begin{gathered}
W_{D W L}(z)=A \cos (n(z+d))+B \sin (n(z+d)) \\
W_{D W L}^{\prime}(z)=-A n \sin (n(z+d))+B n \cos (n(z+d))
\end{gathered}
$$

In the remnant mixed layer (RML):

$$
\begin{gathered}
W_{R M L}(z)=C \cosh (\mu(z+d))+D \sinh (\mu(z+d)) \\
W_{R M L}^{\prime}(z)=C \mu \sinh (\mu(z+d))+D \mu \cosh (\mu(z+d))
\end{gathered}
$$

In the stratified interior (SI):

$$
\begin{gathered}
W_{S I}(z)=E \cos (m(z+H))+F \sin (m(z+H)) \\
W_{S I}^{\prime}(z)=-E m \sin (m(z+H))+F m \cos (m(z+H))
\end{gathered}
$$

where

$$
n^{2}=\alpha^{2} \frac{N_{d}^{2}-\omega^{2}}{\omega^{2}-f^{2}},
$$


and

$$
\mu^{2}=\alpha^{2} \frac{\omega^{2}}{\omega^{2}-f^{2}},
$$

and

$$
m^{2}=\alpha^{2} \frac{N_{0}^{2}-\omega^{2}}{\omega^{2}-f^{2}}
$$

We can relate the coefficients in the DWL with the stratified interior, using the boundary and matching conditions.

\section{B.1.1 Surface condition}

We impose a rigid lid. Therefore, vertical velocity must go to zero at the surface.

$$
W_{D W L}(0)=0=A \cos (n d)+B \sin (n d)
$$

And thus:

$$
A=-B \tan (n d)
$$

\section{B.1.2 Base of diurnal warm layer}

At the base of the diurnal warm layer (the top of the remnant mixed layer) we want vertical velocity and pressure to be continuous. We have two conditions, the first of which is that vertical velocity is continuous across the boundary, which is simply:

$$
W_{D W L}(-d)=W_{R M L}(-d)
$$

Thus:

$$
A=C
$$

The second conditions is that pressure is continuous across the interface, thus,

$$
W_{D W L}^{\prime}(-d)=W_{R M L}^{\prime}(-d)
$$

Thus:

$$
B n=D \mu
$$




\section{B.1.3 Seasonal Thermocline}

At the seasonal thermocline we want vertical velocity and pressure to be continuous. The seasonal thermocline is the base of the mixed layer. We have two conditions, the first of which is simply:

$$
W_{R M L}(-H)=W_{S I}(-H)
$$

Thus:

$$
C \cosh (-\mu(H-d))+D \sinh (-\mu(H-d))=E
$$

The second conditions is that pressure must be continuous across the boundary, which is

$$
\int_{-H-\epsilon}^{-H} \frac{d^{2} W}{d z^{2}}+\alpha^{2} \frac{N^{2}-\omega^{2}}{\omega^{2}-f^{2}} W d z=0
$$

where $\epsilon$ is an infinitesimally small distance. The buoyancy frequency profile can be expressed as

$$
N^{2}(z)=N_{d}^{2}(1-\mathcal{H}(-z-d))+N_{0}^{2} \mathcal{H}(-z-H)+\frac{g^{\prime}}{\epsilon} \delta(z+H)
$$

where $\delta$ is the Dirac delta function, $\mathcal{H}$ is the Heavyside function (integral of $\delta$ function), and $g^{\prime}=g \Delta \rho / \rho_{0}$. When $\epsilon \rightarrow 0$, this becomes:

$$
W_{R M L}^{\prime}(-H)-W_{S I}^{\prime}(-H)+\alpha^{2} \frac{g^{\prime}}{\omega^{2}-f^{2}} W_{S I}(-H)=0
$$

and thus:

$$
C \mu \sinh (-\mu(H-d))+D \mu \cosh (-\mu(H-d))-F m+\alpha^{2} \frac{g^{\prime}}{\omega^{2}-f^{2}} E=0
$$

\section{B.1.4 Horizontal kinetic energy of interior}

We want to look at the DWL in terms of the stratified interior, so that we can see what wavelengths and frequencies have enough energy to generate strong shear in the upper ocean. We can use our five conditions (Eq. B.13, B.15, B.17, B.19, and B.23) to write E and F and in terms of A. Using Eq. B.17, we know that:

$$
D \mu=B n
$$


and that $B=-A / \tan (n d)$ from Eq. B.13, thus:

$$
D \mu=-\frac{A n}{\tan (n d)}
$$

We can use the above relationship, the fact that $A=C$ from Eq. B.15, and start with Eq. B.19 to get

$$
E=A \cosh (-\mu(H-d))-\frac{A n}{\mu \tan (n d)} \sinh (-\mu(H-d))
$$

which can be written as:

$$
E=\frac{A}{d}\left[d \cosh (\mu(H-d))-\frac{n d}{\mu \tan (n d)} \sinh (\mu(H-d))\right]
$$

Starting with Eq. B.23,

$$
F m=C \mu \sinh (-\mu(H-d))+D \mu \cosh (-\mu(H-d))+\alpha^{2} \frac{g^{\prime}}{\omega^{2}-f^{2}} E
$$

Thus using $C=A$ and the relationships for $D$ and $E$ in terms of $A$ from above, we have:

$$
\begin{gathered}
F m=A \mu \sinh (-\mu(H-d))-\frac{A n}{\tan (n d)} \cosh (-\mu(H-d)) \\
+\alpha^{2} \frac{g^{\prime}}{\omega^{2}-f^{2}} \frac{A}{d}\left(d \cosh (-\mu(H-d))-\frac{n d}{\mu \tan (n d)} \sinh (-\mu(H-d))\right)
\end{gathered}
$$

and

$$
\begin{gathered}
F m=\frac{A}{d}\left[-\mu d \sinh (\mu(H-d))-\frac{n d}{\tan (n d)} \cosh (\mu(H-d))\right. \\
\left.+\frac{\alpha^{2}}{\omega^{2}-f^{2}} g^{\prime}\left(d \cosh (\mu(H-d))+\frac{n d}{\mu \tan (n d)} \sinh (\mu(H-d))\right)\right]
\end{gathered}
$$

We need to calculate the horizontal kinetic energy of the stratified interior. Using Eq. B.27 and B.30, we will define the part in parenthesis as $P_{E}$ and $P_{F}$, respectively. Thus, we can write:

$$
W_{S I}^{\prime}(z)=-\frac{A}{d} P_{E} m \sin (m(z+H))+\frac{A}{d} P_{F} \cos (m(z+H))
$$

and

$$
\left\langle W_{S I}^{\prime 2}\right\rangle=\frac{m}{2 \pi} \int_{-H-\frac{m}{2 \pi}}^{-H}\left(W_{S I}^{\prime}\right)^{2} d z
$$




$$
\begin{gathered}
=\frac{m}{2 \pi} \int_{-H-\frac{m}{2 \pi}}^{-H}\left(-\frac{A}{d} P_{E} m \sin (m(z+H))+\frac{A}{d} P_{F} \cos (m(z+H))\right)^{2} d z \\
=\frac{A^{2} m}{d^{2} 2 \pi} \int_{-H-\frac{2 \pi}{m}}^{-H}\left(-P_{E} m \sin (m(z+H))+P_{F} \cos (m(z+H))\right)^{2} d z \\
=\frac{A^{2} m}{d^{2} 2 \pi}\left(\pi m P_{E}^{2}+\frac{\pi P_{F}^{2}}{m}\right) \\
=\frac{A^{2}}{2 d^{2}}\left(m^{2} P_{E}^{2}+P_{F}^{2}\right) .
\end{gathered}
$$

Putting back in our $P_{E}$ and $P_{F}$, we have:

$$
\left\langle W_{S I}^{\prime 2}\right\rangle=\frac{A^{2}}{2 d^{2}}\left[m^{2} H_{*}^{2}+\left(\mu d_{*}-\frac{\alpha^{2}}{\omega^{2}-f^{2}} g^{\prime} H_{*}\right)^{2}\right]
$$

where

$$
H_{*}=d\left(\cosh (\mu(H-d))+\frac{n \sinh (\mu(H-d))}{\mu \tan (n d)}\right)
$$

and

$$
d_{*}=d\left(\sinh (\mu(H-d))+\frac{n \cosh (\mu(H-d))}{\mu \tan (n d)}\right)
$$

\section{B.1.5 Mean horizontal kinetic energy of DWL}

The mean horizontal kinetic energy can be related to the first derivative (in $z$ ) squared (we neglect the oscillatory part in horizontal space and time as that will cancel when we compare it to the horizontal KE of the interior).

$$
\left\langle W_{D W L}^{\prime 2}\right\rangle=\frac{1}{d} \int_{-d}^{0} W_{D W L}^{\prime 2} d z=\frac{1}{d} \int_{-d}^{0}(-A n \sin (n(z+d))+B n \cos (n(z+d)))^{2} d z
$$

Noting that $B=-A / \tan (n d)$, we can write the above equation as

$$
\begin{array}{r}
\left\langle W_{D W L}^{\prime 2}\right\rangle=\frac{A^{2} n^{2}}{d} \int_{-d}^{0}\left(\sin (n(z+d))+\frac{1}{\tan (n d)} \cos (n(z+d))\right)^{2} d z \\
\left\langle W_{D W L}^{\prime 2}\right\rangle=\frac{A^{2} n^{2}}{d} \frac{\sin (2 n d)+2 n d}{4 n \sin ^{2}(n d)}=\frac{A^{2}}{d^{2}} \frac{n d \sin (2 n d)+2 n^{2} d^{2}}{4 \sin ^{2}(n d)}
\end{array}
$$




\section{B.1.6 Relationship of DWL and interior KE}

Using the results in the previous two section, we can write the mean horizontal kinetic energy of the diurnal warm layer, $\left\langle U_{D W L}^{2}\right\rangle$, and interior, $\left\langle U_{S I}^{2}\right\rangle$, as

$$
\frac{\left\langle U_{D W L}^{2}\right\rangle}{\left\langle U_{S I}^{2}\right\rangle}=\frac{2 n^{2} d^{2}+n d \sin (2 n d)}{2 \sin ^{2}(n d)}\left[m^{2} H_{*}^{2}+\left(\mu d_{*}-\frac{\alpha^{2}}{\omega^{2}-f^{2}} g^{\prime} H_{*}\right)^{2}\right]^{-1}
$$

Now, let's look at a limit where the near-surface stratification exists to the seasonal thermocline $(d \rightarrow H)$. If the upper layer is assumed to be unstratified $(n \rightarrow i \mu)$, and we assume that $\mu H \ll 1$ as was done in D'Asaro (1978), we get

$$
\frac{\left\langle U_{D W L}^{2}\right\rangle}{\left\langle U_{S I}^{2}\right\rangle}=2\left[m^{2} H^{2}+\left(1-\frac{\alpha^{2}}{\omega^{2}-f^{2}} g^{\prime} H\right)^{2}\right]^{-1}
$$

which is the D'Asaro (1978) solution. There is a factor of two difference between the result shown here and the results of D'Asaro (1978). However, given the lack of detail in D'Asaro (1978), the difference may be due to an assumption difference not clearly stated. The factor of 2 difference is consistent throughout the problem and does not change the underlying physics of the problem at hand.

\section{B.1.7 Internal wave induced Richardson number instability}

Deep internal waves that do completely decay over a depth less than the depth of the remnant mixed layer can manifest in the diurnal warm layer as propagating waves. Certain waves (in frequency and wavenumber space) under certain conditions (vertical structure of stratification) can generate strong shear across the diurnal warm layer. Here, we are neglecting any shear that may exist across the diurnal warm layer due to wind-drive shear (no mean flow). We want to explore this setup in terms of an internal wave spectrum like D'Asaro (1978) did with the Desaubies (1976) spectrum. Here, we use the Garrett and Munk spectrum. The Richardson number is defined as:

$$
R i=\frac{N_{d}^{2}}{\vec{u}_{z}^{2}}
$$


where:

$$
\vec{u}_{z}^{2}=u_{z}^{2}+v_{z}^{2}=\frac{W^{\prime \prime}(z)^{2}}{\alpha^{2}}\left[\left(i e^{i(\alpha x-\omega t)}\right)^{2}+\left(\frac{f}{\omega} e^{i(\alpha x-\omega t)}\right)^{2}\right]
$$

The real part of the bracketed portion of the equation (taking the real part before squaring) can be expressed as:

$$
\left(i e^{i(\alpha x-\omega t)}\right)^{2}+\left(\frac{f}{\omega} e^{i(\alpha x-\omega t)}\right)^{2}=\sin ^{2}(\alpha x-\omega t)+\frac{f^{2}}{\omega^{2}} \cos ^{2}(\alpha x-\omega t)
$$

Since we are concerned with the minimum possible Richardson number, we want to know the maximum shear. We can set the oscillatory part of the solution in $x$ and $t$ to unity, therefore:

$$
\vec{u}_{z}^{2}=\frac{W^{\prime \prime}(z)^{2}}{\alpha^{2}}
$$

The second derivative the vertical structure of the vertical velocity is:

$$
W_{D W L}^{\prime \prime}(z)=-A n^{2} \cos (n(z+d))+\frac{A n^{2}}{\tan (n d)} \sin (n(z+d))
$$

Thus, shear can be written as:

$$
\vec{u}_{z}^{2}=\frac{A^{2} n^{4}}{\alpha^{2}}\left(\cos (n(z+d))-\frac{\sin (n(z+d))}{\tan (n d)}\right)^{2}
$$

We know or prescribe all variables except for the value of $A^{2}$, which we can get from using an internal wave spectrum for the deep ocean through the relationship for the mean horizontal kinetic energy for the stratified interior. We now need to worry about the oscillatory portion of the wave in horizontal space and time. The average horizontal kinetic energy in the stratified interior (including the oscillatory portion in space and time, see Eq. B.43) is:

$$
\left\langle U_{S I}^{2}\right\rangle=\frac{1}{4}\left(\frac{f^{2}}{\omega^{2}}+1\right)\left\langle W_{S I}^{\prime 2}\right\rangle
$$

And thus using the above equation and Eq. B.33:

$$
\left\langle U_{S I}^{2}\right\rangle=\frac{A^{2}}{8 d^{2}}\left(\frac{f^{2}}{\omega^{2}}+1\right)\left[m^{2} H_{*}^{2}+\left(\mu d_{*}-\frac{\alpha^{2}}{\omega^{2}-f^{2}} g^{\prime} H_{*}\right)^{2}\right]
$$


Now, we use the Garrett and Munk spectrum (Garrett and Munk, 1972, 1975, 1979) as the "known" value of the mean horizontal kinetic energy of the stratified interior. The horizontal kinetic energy at a specific wavenumber and frequency can be written as:

$$
\left\langle U_{G M}^{2}\right\rangle=N_{0} b^{2} N \frac{\omega^{2}+f^{2}}{\omega^{2}} E(\alpha, \omega)
$$

where $N_{0}$ is the scaling quantity of the buoyancy frequency of at the top of the thermocline (3 cph), $b$ is the scaling quantity of the buoyancy scale depth $(1.3 \mathrm{~km}), N$ is the local buoyancy frequency, and $E(\alpha, \omega)$ is the energy spectrum as a function of wavenumber and frequency. We invite the reader to read Garrett and Munk $(1972,1975,1979)$ and Munk (1981) for the details of the spectrum. Now, we can find $A^{2}$ for a given wavenumber and frequency.

$$
A(\alpha, \omega)^{2}=8 d^{2}\left\langle U_{G M}^{2}\right\rangle\left(\frac{\omega^{2}}{\omega^{2}+f^{2}}\right)\left[m^{2} H_{*}^{2}+\left(\mu d_{*}-\frac{\alpha^{2}}{\omega^{2}-f^{2}} g^{\prime} H_{*}\right)^{2}\right]^{-1}
$$

In addition, the maximum amplitude of the oscillatory portion of the shear term is found analytically:

$$
F_{\text {max }}=\operatorname{MAX}\left[\left(\cos (n(z+d))-\frac{\sin (n(z+d))}{\tan (n d)}\right)^{2}\right]
$$

Therefore,

$$
\operatorname{MAX}\left(\vec{u}_{z}^{2}\right)=\frac{A^{2} n^{4}}{\alpha^{2}} F_{\max }
$$

This maximum for each frequency and wavenumber can be used to estimate the likelihood of shear induced by deep internal waves causing the $R i<0.25$. 


\section{Bibliography}

Abernathey, R., and J. Marshall, 2013: Global surface eddy diffusivities derived from satellite altimetry. Journal of Geophysical Research, 118 (2), 901-916.

Adler, R. F., and Coauthors, 2003: The version-2 Global Precipitation Climatology Project (GPCP) monthly precipitation analysis (1979-present). Journal of Hydrometeorology, 4 (6), 1147-1167.

Alpers, W., 1985: Theory of radar imaging of internal waves. Nature, 314, 245-247.

Babanin, A., 2006: On a wave-induced turbulence and a wave-mixed upper ocean layer. Geophysical Research Letters, 33 (20).

Beare, R. J., and Coauthors, 2006: An intercomparison of large-eddy simulations of the stable boundary layer. Boundary-Layer Meteorology, 118 (2), 247-272.

Belcher, S. E., and Coauthors, 2012: A global perspective on Langmuir turbulence in the ocean surface boundary layer. Geophysical Research Letters, 39 (18).

Bergmann, J. C., 2011: Comment on local similarity in the stable boundary layer and mixing-length approaches: Consistency of concepts. Boundary-Layer Meteorology, 138 (2), 337-340.

Bernie, D., E. Guilyardi, G. Madec, J. Slingo, S. Woolnough, and J. Cole, 2008: Impact of resolving the diurnal cycle in an ocean-atmosphere GCM. Part 2: A diurnally coupled CGCM. Climate Dynamics, 31 (7-8), 909-925.

Bernie, D., S. Woolnough, J. Slingo, and E. Guilyardi, 2005: Modeling diurnal and intraseasonal variability of the ocean mixed layer. Journal of Climate, 18 (8), 1190-1202.

Bogdanoff, A. S., C. A. Clayson, J. L. Brown, and J. B. Roberts, In Preparation: Estimation of sea surface diurnal warming. Journal of Geophysical Research.

Booker, J. R., and F. P. Bretherton, 1967: The critical layer for internal gravity waves in a shear flow. Journal of Fluid Mechanics, 27, 513-539.

Bracchini, L., A. M. Dattilo, H. Vincent, S. A. Loiselle, A. Tognazzi, and C. Rossi, 2009: Modelling upwelling irradiance using secchi disk depth in lake ecosystems. Journal of Limnology, 68 (1), 83-91.

Brainerd, K. E., and M. C. Gregg, 1993a: Diurnal restratification and turbulence in the oceanic surface mixed layer: 1. Observations. Journal of Geophysical Research, 98 (C12), 22 645-22 656, doi:10.1029/93JC02297. 
Brainerd, K. E., and M. C. Gregg, 1993b: Diurnal restratification and turbulence in the oceanic surface mixed layer: 2. Modeling. Journal of Geophysical Research, 98 (C12), 22 657-22 664, doi:10.1029/93JC02298.

Callaghan, A. H., B. Ward, and J. Vialard, 2014: Influence of surface forcing on near-surface and mixing layer turbulence in the tropical indian ocean. Deep Sea Research Part I, 94, $107-123$.

Callies, J., and R. Ferrari, 2013: Interpreting energy and tracer spectra of upper-ocean turbulence in the submesoscale range (1-200 km). Journal of Physical Oceanography, 43 (11), 2456-2474.

Callies, J., R. Ferrari, J. M. Klymak, and J. Gula, 2015: Seasonality in submesoscale turbulence. Nature Communications, 6.

Cheng, Y., M. B. Parlange, and W. Brutsaert, 2005: Pathology of Monin-Obukhov similarity in the stable boundary layer. Journal of Geophysical Research, 110 (D6).

Clayson, C. A., and A. S. Bogdanoff, 2013: The effect of diurnal sea surface temperature warming on climatological air-sea fluxes. Journal of Climate, 26 (8), 2546-2556.

Clayson, C. A., and L. H. Kantha, 1999: Turbulent kinetic energy and its dissipation rate in the equatorial mixed layer. Journal of Physical Oceanography, 29 (9), 2146-2166.

Clayson, C. A., J. B. Roberts, and A. S. Bogdanoff, 2016a: Seaflux version 1: A new satellite-based ocean-atmosphere turbulent flux dataset. International Journal of Climate, In Review.

Clayson, C. A., L. C. St. Laurent, J. T. Farrar, and A. S. Bogdanoff, 2016b: Enhanced dissipation in the upper ocean during diurnal warming. Nature Geoscience, In Preparation.

Clayson, C. A., and D. Weitlich, 2005: Diurnal warming in the tropical Pacific and its interannual variability. Geophysical Research Letters, 32 (21).

Clayson, C. A., and D. Weitlich, 2007: Variability of tropical diurnal sea surface temperature. Journal of Climate, 20 (2), 334-352.

Colbo, K., and R. A. Weller, 2009: Accuracy of the IMET sensor package in the subtropics. Journal of Atmospheric and Oceanic Technology, 26 (9), 1867-1890.

Craig, P. D., and M. L. Banner, 1994: Modeling wave-enhanced turbulence in the ocean surface layer. Journal of Physical Oceanography, 24 (12), 2546-2559.

Curry, J. A., and Coauthors, 2004: Seaflux. Bulletin of the American Meteorological Society, 85 (3), 409-424.

Danabasoglu, G., W. G. Large, J. J. Tribbia, P. R. Gent, B. P. Briegleb, and J. C. McWilliams, 2006: Diurnal coupling in the tropical oceans of CCSM3. Journal of climate, 19 (11), 2347-2365.

Daniel, T., J. Manley, and N. Trenaman, 2011: The wave glider: Enabling a new approach to persistent ocean observation and research. Ocean Dynamics, 61 (10), 1509-1520. 
D'Asaro, E., J. Thomson, A. Shcherbina, R. Harcourt, M. Cronin, M. Hemer, and B. FoxKemper, 2014: Quantifying upper ocean turbulence driven by surface waves. Geophysical Research Letters, 41 (1), 102-107.

D'Asaro, E. A., 1978: Mixed layer velocities induced by internal waves. Journal of Geophysical Research, 83 (C5), 2437-2438.

Dash, P., A. Ignatov, Y. Kihai, and J. Sapper, 2010: The SST quality monitor (SQUAM). Journal of Atmospheric and Oceanic Technology, 27 (11), 1899-1917.

Desaubies, Y. J. F., 1976: Analytical representation of internal wave spectra. Journal of Physical Oceanography, 6 (6), 976-981.

Dickey, T. D., and J. J. Simpson, 1983: The influence of optical water type on the diurnal response of the upper ocean. Tellus $B, 35$ (2).

Donlon, C., and Coauthors, 2007: The Global Ocean Data Assimilation Experiment Highresolution Sea Surface Temperature Pilot Project. Bulletin of the American Meteorological Society, 88 (8), 1197.

Drushka, K., S. T. Gille, and J. Sprintall, 2014: The diurnal salinity cycle in the tropics. Journal of Geophysical Research, 119 (9), 5874-5890.

Durack, P. J., and S. E. Wijffels, 2010: Fifty-year trends in global ocean salinities and their relationship to broad-scale warming. Journal of Climate, 23 (16), 4342-4362, doi: 10.1175/2010JCLI3377.1, URL http://dx.doi.org/10.1175/2010JCLI3377.1.

Durack, P. J., S. E. Wijffels, and R. J. Matear, 2012: Ocean salinities reveal strong global water cycle intensification during 1950 to 2000. Science, 336 (6080), 455-458.

Edson, J., and Coauthors, 2007: The Coupled Boundary Layers and Air-Sea Transfer experiment in low winds. Bulletin of the American Meteorological Society, 88 (3), 341-356.

Edson, J. B., and C. W. Fairall, 1998: Similarity relationships in the marine atmospheric surface layer for terms in the TKE and scalar variance budgets. Journal of the Atmospheric Sciences, 55 (13), 2311-2328.

Edson, J. B., A. A. Hinton, K. E. Prada, J. E. Hare, and C. W. Fairall, 1998: Direct covariance flux estimates from mobile platforms at sea. Journal of Atmospheric and Oceanic Technology, 15 (2), 547-562.

Embury, O., C. J. Merchant, and G. K. Corlett, 2012: A reprocessing for climate of sea surface temperature from the along-track scanning radiometers: Initial validation, accounting for skin and diurnal variability effects. Remote Sensing of Environment, 116, 62-78.

Fairall, C. W., E. F. Bradley, J. S. Godfrey, G. A. Wick, J. B. Edson, and G. S. Young, 1996: Cool-skin and warm-layer effects on sea surface temperature. Journal of Geophysical Research, 101 (C1), 1295-1308, doi:0.1029/95JC03190.

Fairall, C. W., E. F. Bradley, J. E. Hare, a. a. Grachev, and J. B. Edson, 2003: Bulk parameterization of air-sea fluxes: Updates and verification for the COARE algorithm. Journal of Climate, 16 (4), 571-591. 
Fairall, C. W., R. Markson, G. E. Schacher, and K. L. Davidson, 1980: An aircraft study of turbulence dissipation rate and temperature structure function in the unstable marine atmospheric boundary layer. Boundary-Layer Meteorology, 19 (4), 453-469.

Farrar, J., and Coauthors, 2015: Salinity and temperature balances at the spurs central mooring during fall and winter. Oceanograpy, 28 (1), 56-65.

Farrar, J. T., C. J. Zappa, R. A. Weller, and A. T. Jessup, 2007: Sea surface temperature signals of oceanic internal waves in low winds. Journal of Geophysical Research, 112 (C06014).

Fer, I., A. K. Peterson, and J. E. Ullgren, 2014: Microstructure measurements from an underwater glider in the turbulent Faroe Bank Channel overflow. Journal of Atmospheric and Oceanic Technology, 31 (5), 1128-1150.

Fernando, H. J. S., and J. C. R. Hunt, 1996: Some aspects of turbulence and mixing in stably stratified layers. Dynamics of Atmospheres and Oceans, 23 (1), 35-62.

Ferrari, R., and W. Young, 1997: On the development of thermohaline correlations as a result of nonlinear diffusive parameterizations. Journal of Marine Research, 55 (6), 1069-1101.

Fisher, A. S., 2000: The upper ocean response to the monsoon of the Arabian Sea. Ph.D. thesis, Massachusetts Institute of Technology and Woods Hole Oceanographic Institution.

Foken, T., 2006: 50 years of the Monin-Obukhov similarity theory. Boundary-Layer Meteorology, 119 (3), 431-447.

Gargett, A. E., P. J. Hendricks, T. Sanford, T. Osborn, and A. Williams, 1981: A composite spectrum of vertical shear in the upper ocean. Journal of Physical Oceanography, 11 (9), $1258-1271$.

Gargett, A. E., T. Osborn, and P. Nasmyth, 1984: Local isotropy and the decay of turbulence in a stratified fluid. Journal of Fluid Mechanics, 144, 231-280.

Garrett, C., and W. Munk, 1972: Space-time scales of internal waves. Geophysical and Astrophysical Fluid Dynamics, 3 (1), 225-264.

Garrett, C., and W. Munk, 1975: Space-time scales of internal waves: A progress report. Journal of Geophysical Research, 80 (3), 291-297.

Garrett, C., and W. Munk, 1979: Internal waves in the ocean. Annual Review of Fluid Mechanics, 11 (1), 339-369.

Gentemann, C. L., P. J. Minnett, P. Le Borgne, and C. J. Merchant, 2008: Multi-satellite measurements of large diurnal warming events. Geophysical Research Letters, 35 (22).

Gentemann, C. L., P. J. Minnett, and B. Ward, 2009: Profiles of Ocean Surface Heating (POSH): A new model of upper ocean diurnal warming. Journal of Geophysical Research, $114(\mathrm{C} 7)$.

Gerkema, T., and J. Zimmerman, 2008: An introduction to internal waves, an introduction to internal waves T. Gerkema J.T.F. Zimmerman Lecture notes, Royal NIOZ.

Gill, A. E., 1982: Atmosphere-Ocean Dynamics. 1st ed., Elsevier. 
Grachev, A. A., E. L. Andreas, C. W. Fairall, P. S. Guest, and P. O. G. Persson, 2013: The critical richardson number and limits of applicability of local similarity theory in the stable boundary layer. Boundary-Layer Meteorology, 147 (1), 51-82.

Gregg, M. C., and M. G. Briscoe, 1979: Internal waves, finestructure, microstructure, and mixing in the ocean. Reviews of Geophysics, 17 (7), 1524-1548.

Hage, E., and A. Tilgner, 2010: High Rayleigh number convection with double diffusive fingers. Physics of Fluids, 22 (7), 076603.

Harcourt, R. R., 2015: An improved second-moment closure model of Langmuir turbulence. Journal of Physical Oceanography, 45 (1), 84-103.

Hodges, B. A., and D. M. Fratantoni, 2014: AUV observations of the diurnal surface layer in the North Atlantic salinity maximum. Journal of Physical Oceanography, 44 (6), 15951604, doi:10.1175/JPO-D-13-0140.1.

Holte, J., and L. Talley, 2009: A new algorithm for finding mixed layer depths with applications to argo data and subantarctic mode water formation. Journal of Atmospheric and Oceanic Technology, 26 (9), 1920-1939.

Hosom, D. S., R. A. Weller, R. E. Payne, and K. E. Prada, 1995: The IMET (Improved Meteorology) ship and buoy systems. Journal of Atmospheric and Oceanic Technology, $12(3), 527-540$.

Houghton, D., 1969: Acapulco '68. Weather, 24 (1), 2-18.

Itsweire, E., J. Koseff, D. Briggs, and J. Ferziger, 1993: Turbulence in stratified shear flows: Implications for interpreting shear-induced mixing in the ocean. Journal of Physical Oceanography, 23 (7), 1508-1522.

Jerlov, N. G., 1951: Optical studies of ocean water. Deep Sea Expedition, 3, 1-19.

Jerlov, N. G., 1976: Marine Optics. Elsevier.

Joyce, R. J., J. E. Janowiak, P. A. Arkin, and P. Xie, 2004: CMORPH: a method that produces global precipitation estimates from passive microwave and infrared data at high spatial and temporal resolution. Journal of Hydrometeorology, 5 (3), 487-503, URL http://dx.doi.org/10.1175/1525-7541(2004) 005<0487:CAMTPG>2 . 0. C0; 2 .

Kantha, L., H. Tamura, and Y. Miyazawa, 2014: Comment on "Wave-turbulence interaction and its induced mixing in the upper ocean" by Huang and Qiao. Journal of Geophysical Research, 119 (2), 1510-1515.

Kantha, L. H., and C. A. Clayson, 1994: An improved mixed layer model for geophysical applications. Journal of Geophysical Research, 99 (C12), 25 235-25 266, doi: 10.1029/94JC02257.

Kantha, L. H., and C. A. Clayson, 2004: On the effect of surface gravity waves on mixing in the oceanic mixed layer. Ocean Modelling, 6 (2), 101-124, doi:10.1016/S14635003(02)00062-8.

Kelly, R., 1967: On the stability of an inviscid shear layer which is periodic in space and time. Journal of Fluid Mechanics, 27 (04), 657-689. 
Kerr, Y. H., and Coauthors, 2010: The SMOS mission: New tool for monitoring key elements of the global water cycle. Proceedings of the IEEE, 98 (5), 666-687.

Kitaigorodskii, S., M. Donelan, J. Lumley, and E. Terray, 1983: Wave-turbulence interactions in the upper ocean. part II. Statistical characteristics of wave and turbulent components of the random velocity field in the marine surface layer. Journal of Physical Oceanography, 13 (11), 1988-1999.

Kouznetsov, R. D., and S. S. Zilitinkevich, 2010: On the velocity gradient in stably stratified sheared flows. Part 2: Observations and models. Boundary-layer meteorology, 135 (3), 513-517.

Kudryavtsev, V. N., and A. V. Soloviev, 1990: Slippery near-surface layer of the ocean arising due to daytime solar heating. Journal of Physical Oceanography, 20 (5), 617-628, doi:10.1175/1520-0485(1990)020<0617:SNSLOT > 2.0.CO;2.

Kumar, P., and M. Sharan, 2012: An analysis for the applicability of Monin-Obukhov similarity theory in stable conditions. Journal of the Atmospheric Sciences, 69 (6), 19101915.

Kundu, P., and I. Cohen, 2008: Fluid mechanics. Elsevier Academic Press, 307.

Large, W., and J. Caron, 2015: Diurnal cycling of sea surface temperature, salinity, and current in the CESM coupled climate model. Journal of Geophysical Research, 120.

Large, W. G., J. C. McWilliams, and S. C. Doney, 1994: Oceanic vertical mixing: A review and a model with a nonlocal boundary layer parameterization. Reviews of Geophysics, 32 (4), 363-403.

Le Vine, D. M., G. S. Lagerloef, F. R. Colomb, S. H. Yueh, and F. A. Pellerano, 2007: Aquarius: An instrument to monitor sea surface salinity from space. Geoscience and Remote Sensing, IEEE Transactions on, 45 (7), 2040-2050.

Leibovich, S., 1983: The form and dynamics of Langmuir circulations. Annual Review of Fluid Mechanics, 15 (1), 391-427.

Li, L., R. W. Schmitt, C. C. Ummenhofer, and K. B. Karnauskas, 2016: North Atlantic salinity as a predictor of Sahel rainfall. Science Advances, 2 (5).

Lighthill, J., 1978: Waves in fluids. Cambridge University Press, 504.

Lindstrom, E., F. Bryan, and R. Schmitt, 2015: SPURS: salinity processes in the upperocean regional study. Oceanography, 28 (1), 14.

Mahrt, L., 1998: Stratified atmospheric boundary layers and breakdown of models. Theoretical and Computational Fluid Dynamics, 11 (3-4), 263-279.

Mahrt, L., 1999: Stratified atmospheric boundary layers. Boundary-Layer Meteorology, 90 (3), 375-396.

Mahrt, L., 2011: The near-calm stable boundary layer. Boundary-Layer Meteorology, 140 (3), 343-360. 
Mahrt, L., 2014: Stably stratified atmospheric boundary layers. Annual Review of Fluid Mechanics, 46, 23-45.

Marshall, J. C., R. G. Williams, and A. G. Nurser, 1993: Inferring the subduction rate and period over the North Atlantic. Journal of Physical Oceanography, 23 (7), 1315-1329.

Mathur, M., and T. Peacock, 2009: Internal wave beam propogation in non-uniform stratifications. Journal of Fluid Mechanics, 639, 133-152.

McNaughton, K., 2006: On the kinetic energy budget of the unstable atmospheric surface layer. Boundary-Layer Meteorology, 118 (1), 83-107.

McPhee, M. G., 1994: On the turbulent mixing length in the oceanic boundary layer. Journal of Physical Oceanography, 24 (9), 2014-2031.

McWilliams, J. C., E. Huckle, and A. F. Shchepetkin, 2009: Buoyancy effects in a stratified ekman layer. Journal of Physical Oceanography, 39 (10), 2581-2599.

McWilliams, J. C., P. P. Sullivan, and C.-H. Moeng, 1997: Langmuir turbulence in the ocean. Journal of Fluid Mechanics, 334, 1-30.

Mellor, G. L., and T. Yamada, 1974: A hierarchy of turbulence closure models for planetary boundary layers. Journal of the Atmospheric Sciences, 31 (7), 1791-1806.

Melnichenko, O., P. Hacker, N. Maximenko, G. Lagerloef, and J. Potemra, 2014: Spatial optimal interpolation of aquarius sea surface salinity: Algorithms and implementation in the North Atlantic. Journal of Atmospheric and Oceanic Technology, 31 (7), 1583-1600.

Monin, A. S., J. L. Lumley, and A. M. IAglom, 1971: Statistical Fluid Mechanics: Mechanics of Turbulence. Vol. 1. MIT press.

Monin, A. S., and A. M. Obukhov, 1954: Osnovnye zakonomernosti turbulentnogo peremeshivanija $\mathrm{v}$ prizemnom sloe atmosfery (Basic laws of turbulent mixing in the atmosphere near the ground). Trudy Geofiz. Inst. AN SSSR, 24 (141), 163-187.

Morris, D. P., H. Zagarese, C. E. Williamson, E. G. Balseiro, B. R. Hargreaves, B. Modenutti, R. Moeller, and C. Queimalinos, 1995: The attenuation of solar UV radiation in lakes and the role of dissolved organic carbon. Limnology and Oceanography, 40 (8), 1381-1391.

Moum, J., J. Klymak, J. Nash, A. Perlin, and W. Smyth, 2007: Energy transport by nonlinear internal waves. Journal of Physical Oceanography, 37 (7), 1968-1988.

Moum, J. N., D. R. Caldwell, and C. A. Paulson, 1989: Mixing in the equatorial surface layer and thermocline. Journal of Geophysical Research, 94 (C2), 2005-2022, doi: 10.1029/JC094iC02p02005.

Moum, J. N., and W. D. Smyth, 2001: Upper ocean mixing processes. Encyclopedia of Ocean Sciences, 6, 3093-3100.

Müller, M., B. K. Arbic, J. G. Richman, J. F. Shriver, E. L. Kunze, R. B. Scott, A. J. Wallcraft, and L. Zamudio, 2015: Toward an internal gravity wave spectrum in global ocean models. Geophysical Research Letters, 42 (9), 3474-3481. 
Munk, W., 1981: Internal waves and small-scale processes. Evolution of Physical Oceanography, 264-291.

Obenour, K. M., 2013: Temporal trends in global sea surface temperature fronts. Ph.D. thesis, University of Rhode Island.

Okubo, A., 1971: Oceanic diffusion diagrams. Deep Sea Research, 18 (8), 789-802.

Osborn, T. R., 1980: Estimates of the local rate of vertical diffusion from dissipation measurements. Journal of Physical Oceanography, 10 (1), 83-89.

Pahlow, M., M. Parlange, and F. Porté-Agel, 2001: On Monin-Obukhov similarity in the stable atmospheric boundary layer. Boundary-Layer Meteorology, 99 (2), 225-248.

Paulson, C. A., and J. J. Simpson, 1981: The temperature difference across the cool skin of the ocean. Journal of Geophysical Research, 86 (11), 4-11.

Pearson, B. C., A. L. Grant, J. A. Polton, and S. E. Belcher, 2015: Langmuir turbulence and surface heating in the ocean surface boundary layer. Journal of Physical Oceanography, 45 (12), 2897-2911.

Peters, H., M. C. Gregg, and T. B. Sanford, 1994: The diurnal cycle of the upper equatorial ocean: Turbulence, fine-scale shear, and mean shear. Journal of Geophysical Research, 99 (C4), 7707-7723.

Peterson, A. K., and I. Fer, 2014: Dissipation measurements using temperature microstructure from an underwater glider. Methods in Oceanography, 10, 44-69.

Phillips, O., 1977: The Dynamics of the Upper Ocean. Cambridge University Press.

Pinkel, R., 1975: Upper ocean internal wave observations from FLIP. Journal of Geophysical Research, 80 (3).

Pinkel, R., and S. Anderson, 1997: Shear, strain, and Richardson number variations in the thermocline. Part I: Statistical description. Journal of Physical Oceanography, 27 (2), $264-281$.

Poje, A. C., and Coauthors, 2014: Submesoscale dispersion in the vicinity of the Deepwater Horizon spill. Proceedings of the National Academy of Sciences, 111 (35), 12 693-12 698.

Poulos, G. S., and Coauthors, 2002: CASES-99: A comprehensive investigation of the stable nocturnal boundary layer. Bulletin of the American Meteorological Society, 83 (4), 555581.

Price, J. F., R. A. Weller, and R. Pinkel, 1986: Diurnal cycling: Observations and models of the upper ocean response to diurnal heating, cooling, and wind mixing. Journal of Geophysical Research, 91 (C7), 8411-8427.

Prytherch, J., J. T. Farrar, and R. A. Weller, 2013: Moored surface buoy observations of the diurnal warm layer. Journal of Geophysical Research, 118 (9), 4553-4569.

Riley, J. J., and Coauthors, 2003: Dynamics of turbulence strongly influenced by buoyancy. Physics of Fluids, 15 (7), 2047-2059. 
Roth, M. W., M. G. Briscoe, and C. McComas III, 1981: Internal waves in the upper ocean. Journal of Physical Oceanography, 11 (9), 1234-1247.

Rotunno, R., 1977: Internal gravity waves in a time-varying stratification. Journal of Fluid Mechanics, 82 (4), 609-619.

Ruddick, B., 1983: A practical indicator of the stability of the water column to double-diffusive activity. Deep Sea Research Part A. Oceanographic Research Papers, 30 (10), 1105 - 1107, URL http://www.sciencedirect.com/science/article/pii/0198014983900638.

Salehipour, H., W. Peltier, and A. Mashayek, 2015: Turbulent diapycnal mixing in stratified shear flows: The influence of prandtl number on mixing efficiency and transition at high reynolds number. Journal of Fluid Mechanics, 773, 178-223.

Saunders, P. M., 1967: The temperature at the ocean-air interface. Journal of the Atmospheric Sciences, 24 (3), 269-273.

Savijärvi, H., 2009: Stable boundary layer: Parametrizations for local and larger scales. Quarterly Journal of the Royal Meteorological Society, 135 (641), 914-921.

Scanlon, B., G. Wick, and B. Ward, 2013: Near-surface diurnal warming simulations: validation with high resolution profile measurements. Ocean Science, 9 (6), 977-986.

Schanze, J. J., R. W. Schmitt, and L. L. Yu, 2010: The global oceanic freshwater cycle: A state-of-the-art quantification. Journal of Marine Research, 68 (3-1), 569-595.

Schmitt, R. W., 1994: Double diffusion in oceanography. Annual Review of Fluid Mechanics, 26 (1), 255-285, URL http://dx.doi.org/10.1146/annurev.fl.26.010194.001351, http://dx.doi.org/10.1146/annurev.fl.26.010194.001351.

Schmitt, R. W., 2008: Salinity and the global water cycle. Oceanography, 21 (1), 12.

Schmitt, R. W., 2011: Thermohaline convection at density ratios below one: A new regime for salt fingers. Journal of Marine Research, 69 (4-5), 779-795.

Schmitt, R. W., P. S. Bogden, and C. E. Dorman, 1989: Evaporation minus precipitation and density fluxes for the North Atlantic. Journal of Physical Oceanography, 19 (9), $1208-1221$.

Schofield, O., and Coauthors, 2007: Slocum gliders: Robust and ready. Journal of Field Robotics, 24 (6), 473-485.

Schudlich, R. R., and J. F. Price, 1992: Diurnal cycles of current, temperature, and turbulent dissipation in a model of the equatorial upper ocean. Journal of Geophysical Research, 97 (C4), 5409-5422.

Seo, H., A. C. Subramanian, A. J. Miller, and N. R. Cavanaugh, 2014: Coupled impacts of the diurnal cycle of sea surface temperature on the Madden-Julian Oscillation. Journal of Climate, 27 (22), 8422-8443.

Sethuraman, S., 1980: A case of persistent breaking of internal gravity waves in the atmospheric surface layer over the ocean. Boundary-Layer Meteorology, 19 (1), 67-80. 
Shay, T. J., and M. Gregg, 1986: Convectively driven turbulent mixing in the upper ocean. Journal of Physical Oceanography, 16 (11), 1777-1798.

Shcherbina, A. Y., and Coauthors, 2015: The LatMix summer campaign: Submesoscale stirring in the upper ocean. Bulletin of the American Meteorological Society, 96 (8), $1257-1279$.

Shinoda, T., 2005: Impact of the diurnal cycle of solar radiation on intraseasonal SST variability in the western equatorial pacific. Journal of Climate, 18 (14), 2628-2636.

Simpson, J., and T. Dickey, 1981: The relationship between downward irradiance and upper ocean structure. Journal of Physical Oceanography, 11 (3), 309-323.

Smyth, W., P. Zavialov, and J. Moum, 1997: Decay of turbulence in the upper ocean following sudden isolation from surface forcing. Journal of physical oceanography, 27 (5), $810-822$.

Smyth, W. D., and J. N. Moum, 2000: Anisotropy of turbulence in stably stratified mixing layers. Physics of Fluids, 12 (6), 1343-1362.

Smyth, W. D., J. N. Moum, L. Li, and S. A. Thorpe, 2013: Diurnal shear instability, the descent of the surface shear layer, and the deep cycle of Equatorial turbulence. Journal of Physical Oceanography, 43 (11), 2432-2455.

Soloviev, A., J. Edson, W. Mcgillis, P. Schluessel, and R. Wanninkof, 2002: Fine thermohaline structure and gas-exchange in the near-surface layer of the ocean during GasEx-98. Gas Transfer at Water Surfaces, 181-185.

Soloviev, A., and R. Lukas, 1997: Observation of large diurnal warming events in the nearsurface layer of the western equatorial Pacific warm pool. Deep Sea Research, 44 (6), $1055-1076$.

Soloviev, A., and R. Lukas, 2006: The Near-Surface Layer of the Ocean. Springer.

Soloviev, A., and R. Lukas, 2014: The Near-Surface Layer of the Ocean. Springer.

Soloviev, A., and N. Vershinsky, 1982: The vertical structure of the thin surface layer of the ocean under conditions of low wind speed. Deep Sea Research, 29 (12), 1437-1449, URL http://www.sciencedirect.com/science/article/pii/0198014982900358.

Soloviev, A., N. Vershinsky, and V. Bezverchnii, 1988: Small-scale turbulence measurements in the thin surface layer of the ocean. Deep Sea Research, 35 (12), 1859-1874, URL http://www.sciencedirect.com/science/article/pii/0198014988901136.

Soloviev, A. V., S. Matt, and A. Fujimura, 2015: Three-dimensional dynamics of freshwater lenses in the ocean's near-surface layer. Oceanography, 28 (1), 142-149.

St. Laurent, L., and R. W. Schmitt, 1999: The contribution of salt fingers to vertical mixing in the North Atlantic Tracer Release Experiment. Journal of Physical Oceanography, 29 (7), 1404-1424.

St. Laurent, L. C., and A. M. Thurnherr, 2007: Intense mixing of lower thermocline water on the crest of the Mid-Atlantic Ridge. Nature, 448 (7154), 680-683. 
Stommel, H., and A. H. Woodcock, 1951: Diurnal heating of the surface of the Gulf of Mexico in the spring of 1942. Eos, Transactions American Geophysical Union, 32 (4), $565-571$.

Stramma, L., P. Cornillon, R. A. Weller, J. F. Price, and M. G. Briscoe, 1986: Large diurnal sea surface temperature variability: Satellite and in situ measurements. Journal of Physical Oceanography, 16 (5), 827-837.

Stuart-Menteth, A. C., I. S. Robinson, and P. G. Challenor, 2003: A global study of diurnal warming using satellite-derived sea surface temperature. Journal of Geophysical Research, 108 (C5).

Sun, J., L. Mahrt, R. M. Banta, and Y. L. Pichugina, 2012: Turbulence regimes and turbulence intermittency in the stable boundary layer during CASES-99. Journal of the Atmospheric Sciences, 69 (1), 338-351.

Sun, O. M., S. R. Jayne, K. L. Polzin, B. A. Rahter, and L. C. St. Laurent, 2013: Scaling turbulent dissipation in the transition layer. Journal of Physical Oceanography, 43 (11), $2475-2489$.

Sundermeyer, M. A., D. A. Birch, J. R. Ledwell, M. Levine, S. Pierce, B. Cervantes, and B. Concannon, In Preparation: Dye dispersion in the seasonal pycnocline of the open ocean at 1-6 day time scales.

Sutherland, B. R., 2006: Rayleigh wave-internal wave coupling and internal wave generation above a model jet stream. Journal of the Atmospheric Sciences, 63 (3), 1042-1055.

Sutherland, B. R., 2010: Internal Gravity Waves. Cambridge University Press.

Sutherland, B. R., and K. Yewchuk, 2004: Internal wave tunnelling. Journal of Fluid Mechanics, 511, 125-134.

Sutherland, G., L. Marie, G. Reverdin, K. H. Christensen, G. Brostrom, and B. Ward, 2016: Enhanced turbulence associated with the diurnal jet in the ocean surface boundary layer. Journal of Physical Oceanography, 46, 3051-3067.

Sutherland, G., G. Reverdin, L. Marié, and B. Ward, 2014: Mixed and mixing layer depths in the ocean surface boundary layer under conditions of diurnal stratification. Geophysical Research Letters, 41 (23), 8469-8476.

Sverdrup, H. U., M. W. Johnson, R. H. Fleming, and Coauthors, 1942: The Oceans: Their physics, chemistry, and general biology, Vol. 7. Prentice-Hall New York.

Taylor, G., 1953: Dispersion of soluble matter in solvent flowing slowly through a tube. Proceedings of the Royal Society of London A: Mathematical, Physical and Engineering Sciences, 219 (1137), 186-203.

Tedford, E. W., S. MacIntyre, S. D. Miller, and M. J. Czikowsky, 2014: Similarity scaling of turbulence in a temperate lake during fall cooling. Journal of Geophysical Research, 119 (8), 4689-4713.

Thorpe, S., 1975: The excitation, dissipation, and interaction of internal waves in the deep ocean. Journal of Geophysical Research, 80 (3), 328-338. 
Thorpe, S., 1978: The near-surface ocean mixing layer in stable heating conditions. Journal of Geophysical Research, 83 (C6), 2875-2885.

Thorpe, S. A., 2005: The Turbulent Ocean. Cambridge University Press.

Tippins, D., and M. Tomczak, 2003: Meridional turner angles and density compensation in the upper ocean. Ocean Dynamics, 53 (4), 332-342.

Tseng, W.-L., B.-J. Tsuang, N. S. Keenlyside, H.-H. Hsu, and C.-Y. Tu, 2014: Resolving the upper-ocean warm layer improves the simulation of the Madden-Julian oscillation. Climate Dynamics, 1-17.

Van de Wiel, B., A. Moene, W. De Ronde, and H. Jonker, 2008: Local similarity in the stable boundary layer and mixing-length approaches: Consistency of concepts. Boundary-Layer Meteorology, 128 (1), 103-116.

Walsh, E. J., R. Pinkel, D. E. Hagan, R. A. Weller, C. W. Fairall, D. P. Rogers, S. P. Burns, and M. Baumgartner, 1998: Coupling of internal waves on the main thermocline to the diurnal surface layer and sea surface temperature during the Tropical Ocean-Global Atmosphere Coupled Ocean-Atmosphere Response Experiment. Journal of Geophysical Research, 103 (C6), 12613-12628.

Wang, D., 2001: Large-eddy simulation of the diurnal cycle of oceanic boundary layer: Sensitivity to domain size and spatial resolution. Journal of Geophysical Research, 106 (C7), 13959-13974.

Ward, B., 2006: Near-surface ocean temperature. Journal of Geophysical Research, 111, 1-18, doi:10.1029/2004JC002689.

Ward, B., T. Fristedt, A. H. Callaghan, G. Sutherland, X. Sanchez, J. Vialard, and A. t. Doeschate, 2014: The Air-Sea Interaction Profiler (ASIP): An autonomous upwardly rising profiler for microstructure measurements in the upper ocean. Journal of Atmospheric and Oceanic Technology, 31 (10), 2246-2267.

Waterman, S., A. C. Naveira Garabato, and K. L. Polzin, 2013: Internal waves and turbulence in the antarctic circumpolar current. Journal of Physical Oceanography, 43 (2), 259-282.

Webster, P. J., C. A. Clayson, and J. A. Curry, 1996: Clouds, radiation, and the diurnal cycle of sea surface temperature in the tropical western pacific. Journal of Climate, 9 (8), $1712-1730$.

Wei, J., and Z. Lee, 2013: Model of the attenuation coefficient of daily photosynthetically available radiation in the upper ocean. Methods in Oceanography, 8, 56-74.

Weihs, R. R., and M. Bourassa, 2014: Modeled diurnally varying sea surface temperatures and their influence on surface heat fluxes. Journal of Geophysical Research, 119 (7), $4101-4123$.

Weller, R., and S. Anderson, 1996: Surface meteorology and air-sea fluxes in the western equatorial pacific warm pool during the TOGA Coupled Ocean-Atmosphere Response Experiment. Journal of Climate, 9 (8), 1959-1990. 
Weller, R. A., and J. F. Price, 1988: Langmuir circulation within the oceanic mixed layer. Deep Sea Research, 35 (5), 711-747.

Wenegrat, J. O., and M. J. McPhaden, 2015: Dynamics of the surface layer diurnal cycle in the equatorial Atlantic Ocean $\left(0^{\circ}, 23^{\circ} \mathrm{W}\right)$. Journal of Geophysical Research: Oceans, 120 (1), 563-581.

Wernand, M., 2010: On the history of the Secchi disc. Journal of the European Optical Society-Rapid publications, $\mathbf{5}$.

Woods, J. D., 1968: An investigation of some physical processes associated with the vertical flow of heat through the upper ocean. Meterological Magazine, 65-72.

Woolnough, S. J., F. Vitart, and M. A. Balmaseda, 2007: The role of the ocean in the Madden-Julian Oscillation: implications for MJO prediction. Quarterly Journal of the Royal Meteorological Society, 133 (622), 117-128.

Wyngaard, J., and O. Coté, 1972: Cospectral similarity in the atmospheric surface layer. Quarterly Journal of the Royal Meteorological Society, 98 (417), 590-603.

Wyngaard, J. C., O. R. Coté, and Y. Izumi, 1971: Local free convection, similarity, and the budgets of shear stress and heat flux. Journal of the Atmospheric Sciences, 28 (7), $1171-1182$.

Yokoyama, R., and M. Konda, 1996: Sea surface effects on the sea surface temperature estimation by remote sensing-Part 2. International Journal of Remote Sensing, 17 (7), 1293-1302, URL http://dx.doi.org/10.1080/01431169608948704, http://dx.doi.org/10.1080/01431169608948704.

Yu, L., 2011: A global relationship between the ocean water cycle and near-surface salinity. Journal of Geophysical Research, 116 (C10).

Zilitinkevich, S. S., T. Elperin, N. Kleeorin, I. Rogachevskii, I. Esau, T. Mauritsen, and M. W. Miles, 2008: Turbulence energetics in stably stratified geophysical flows: Strong and weak mixing regimes. Quarterly Journal of the Royal Meteorological Society, 134 (633), 793-799. 